\title{
Fórmulas da Área e Coárea em Conjuntos Retificáveis
}

\author{
Emma Alejandra Cupitra Vergara \\ DisSERTAÇÃo/TESE APRESENTADA \\ $\mathrm{AO}$ \\ Instituto de Matemática e Estatística \\ DA \\ Universidade De SÃo Paulo \\ PARA \\ OBTENÇÃO DO TÍTULO \\ DE \\ Mestre em CiÊnCias \\ Programa: Matemática \\ Orientador: Prof. Dr. Glaucio Terra
}

Durante o desenvolvimento deste trabalho o autor recebeu auxílio financeiro da CNPq São Paulo, Novembro de 2020 


\section{Formulas da Área e Coárea em Conjuntos Retificáveis}

Esta é a versão original da dissertação/tese elaborada pelo candidato (Emma Alejandra Cupitra Vergara), tal como submetida à Comissão Julgadora. 


\section{Formula de Área e Coarea em conjuntos Retificáveis}

Esta versão da dissertação/tese contém as correções e alterações sugeridas pela Comissão Julgadora durante a defesa da versão original do trabalho, realizada em 20/11/2020. Uma cópia da versão original está disponível no Instituto de Matemática e Estatística da Universidade de São Paulo.

Comissão Julgadora:

- Prof $^{\mathrm{a}}$. Dr ${ }^{\mathrm{a}}$. Nome Completo (orientadora) - IME-USP [sem ponto final]

- Prof. Dr. Nome Completo - IME-USP [sem ponto final]

- Prof. Dr. Nome Completo - IMPA [sem ponto final] 


\section{Agradecimentos}

Primeiramente agradeço a Deus, aos meus pais por estarem no meu processo de formação acadêmica e pessoal, também ao meu orientador o professor Gláucio Terra, pela paciência e apoio que tem me dado até o momento.

Me sinto privilegiada de fazer esse curso em uma institução muito bem conceituada a USP, avançando cada veis mais pelo caminho das Matemáticas, igualmente á CNPq pelo financiamento durante meu processo. E agora por ultimo uma frase boa: seja feliz, é o mais importante! 


\section{Resumo}

Cupitra, E. A. V. Formula de Área e Coarea em conjuntos Retificáveis. 2020. 60 f. Tese (Mestrado) - Instituto de Matemática e Estatística, Universidade de São Paulo, São Paulo, 2020.

A fórmula da área para aplicações Lipschitz em espaços euclidianos é uma generalização do teorema de mudança de variáveis clássico no contexto de integrais múltiplas do Cálculo Avançado. A fórmula da coárea, por sua vez, é uma generalização do teorema de Fubini-Tonelli para medidas produto. Neste trabalho são estudadas versões abstratas destas fórmulas para aplicações Lipschitz definidas em subconjuntos retificáveis de espaços euclidianos.

Palavras-chave: fórmula da área, fórmula da coárea, conjuntos retificáveis. 


\section{Abstract}

Cupitra, E. A. V. Formula of the area and Corea in rectifiable sets. 2020. 120 f. Tese (Mestrado) - Instituto de Matemática e Estatística, Universidade de São Paulo, São Paulo, 2020.

The area formula for Lipschitz maps in Euclidean spaces is a generalization of the classical change of variables theorem for multiple integrals from Advanced Calculus. The coarea formula, in its turn, is a generalization of Fubini-Tonelli's theorem for product measures. In this work, we study abstract versions of those formulae for Lipschitz maps defined on rectifiable subsets of Euclidean spaces.

Keywords: area formula, coarea formula, rectifiable sets. 


\section{Sumário}

$\begin{array}{ll}\text { Lista de Abreviaturas } & \text { ix }\end{array}$

Lista de Símbolos $\quad$ xi

Lista de Figuras $\quad$ xv

1 Introdução $\quad 1$

2 Preâmbulo $\quad 3$

2.1 Medidas e Mensurabilidade . . . . . . . . . . . . . . . . . . . . . . 3

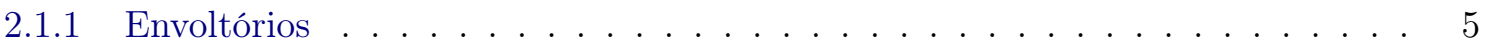

2.1 .2 Operações com Medidas . . . . . . . . . . . . . . . . . 5

2.1 .3 Medidas em Espaços Topológicos . . . . . . . . . . . . . . . . . . . . . 6

2.1 .4 Aplicações Mensuráveis . . . . . . . . . . . . . . . . . . . 8

2.2 Teoria de Integração $\ldots \ldots \ldots \ldots$

2.2 .1 Integrais Superior e Inferior . . . . . . . . . . . . . . . . . 9

2.2.2 Medidas Produto e Teorema de Fubini-Tonelli . . . . . . . . . . . . . . . . . . 10

2.3 Teorema de Severini-Egorov . . . . . . . . . . . . . . . . . . . . . . 11

2.4 Construção de Carathéodory e Medidas de Hausdorff . . . . . . . . . . . . . . . . . . 12

2.5 Teoremas de Cobertura . . . . . . . . . . . . . . . . . . . . . . . . 14

2.5.1 Teorema de Cobertura de Vitali . . . . . . . . . . . . . . . . . . . . . . . . . . 14

2.5.2 Teorema de Cobertura de Besicovitch . . . . . . . . . . . . . . . . 15

2.6 Teoremas de Densidade e Diferenciação de Medidas . . . . . . . . . . . . . . . . 16

2.6 .1 Densidades . . . . . . . . . . . . . . . . . . . . . . . 16

2.6.2 Densidades Relativas e Teoremas de Diferenciação . . . . . . . . . . . . . . . 16

2.7 Medidas de Radon a Valores Vetoriais . . . . . . . . . . . . . . . . . . . . . 18

2.7.1 Convergência Fraca-Estrela . . . . . . . . . . . . . . . . . . . . . . 20

2.8 Algumas Propriedades de Aplicações Lipschitz . . . . . . . . . . . . . . . . . . . . . . 21

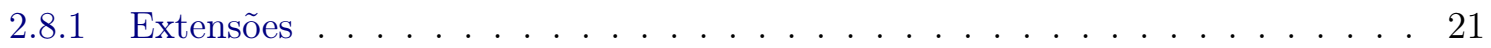

2.8 .2 Diferenciabilidade . . . . . . . . . . . . . . . . . . . 21

2.9 Jacobianos . . . . . . . . . . . . . . . . . . . . . . . . 22

3 Fórmulas da Área e Coárea em Espaços Euclidianos $\quad 25$

3.1 Linearização de Aplicações Lipschitz . . . . . . . . . . . . . . . . . . . . . 25

3.2 Fórmula da Área em Espaços Euclidianos . . . . . . . . . . . . . . . . . . . 27

3.2 .1 Aplicações da Fórmula da Área . . . . . . . . . . . . . . . . . . . . . . . 34 
3.3 Fórmula da Coárea em Espaços Euclidianos . . . . . . . . . . . . . . . . . . . 36

3.3 .1 Aplicações da Fórmula da Coárea . . . . . . . . . . . . . . . . . . . . . . . . 48

4 Retificabilidade $\quad 51$

4.1 Conjuntos Retificáveis . . . . . . . . . . . . . . . . . . . . . . . . . 51

4.2 Um Teorema de Estrutura para Conjuntos Retificáveis . . . . . . . . . . . . . . . 53

4.3 Propriedades Tangenciais . . . . . . . . . . . . . . . . . . 55

4.4 Retificabilidade a partir de blow-ups de medidas de Radon . . . . . . . . . . . . . . . 62

5 Formula da Área e Coárea em Conjuntos Retificáveis $\quad 69$

5.1 Fórmula da Área para Conjuntos Retificáveis . . . . . . . . . . . . . . . . . . . 69

5.2 Fórmula da Coárea para Conjuntos Retificáveis . . . . . . . . . . . . . . . . . . 74

$\begin{array}{ll}\text { Referências Bibliográficas } & 81\end{array}$ 


\section{Lista de Abreviaturas}

SVP Propriedade de Vitali simétrica (symmetric Vitali property)

$L D \quad$ Decomposição de Lebesgue (Lebesgue decomposition)

$F A \quad$ Fórmula da área (area formula)

FCA Fórmula da coárea (coarea formula)

TCM Teorema da convergência monótona (monotone convergence theorem)

$T C D$ Teorema da convergência dominada, de Lebesgue (Lebesgue dominated convergence theorem)

$T M V$ Teorema de mudança de variáveis (change of variables theorem) 


\section{Lista de Símbolos}

\begin{tabular}{|c|c|}
\hline $\mathscr{P}(X)$ ou $2^{X}$ & conjunto das partes do conjunto $X$ \\
\hline$N \triangle M$ & diferença simétrica entre $N$ e $M$ \\
\hline $\mathbb{B}(x, r)$ & bola fechada de centro $x$ e raio $r$ \\
\hline $\mathbb{U}(x, r)$ & bola aberta de centro $x$ e raio $r$ \\
\hline $\mathbb{B}^{m}$ & bola fechada unitária em $\mathbb{R}^{m}$ \\
\hline $\mathbb{S}^{m-1}$ & esfera unitária em $\mathbb{R}^{m}$ \\
\hline$\alpha(m)$ & é o volume (i.e. medida de Lebesgue) da bola unitária em $\mathbb{R}^{m}$ \\
\hline $\mathscr{B}_{X}$ & $\sigma$-álgebra de Borel do espaço topológico $X$ \\
\hline $\mathscr{L}$ & $\sigma$-álgebra de Lebesgue \\
\hline$\sigma(\mu)$ & $\sigma$-álgebra dos conjuntos mensuráveis com respeito à medida exterior $\mu$ \\
\hline$\delta_{a}$ & medida de Dirac centrada em $a$ \\
\hline $\mathcal{L}^{n}$ & medida de Lebesgue em $\mathbb{R}^{n}$ \\
\hline$|E|$ & medida de Lebesgue de $E$ \\
\hline $\mathcal{H}^{n}$ & medida de Hausdorff $n$-dimensional \\
\hline $\mathcal{H}$-dim $A$ & dimensão de Hausdorff de A \\
\hline $\mathcal{H}_{\delta}^{m}$ & $\delta$-aproximação da medida de Hausdorff $m$-dimensional \\
\hline $\int_{*} f$ & integral inferior de $f$ \\
\hline $\int^{*} f$ & integral superior de $f$ \\
\hline $\mathrm{L}^{+}(\mu)$ & funções $\mu$-mensuráveis tomando valores em $[0, \infty]$ \\
\hline $\mathrm{L}^{1}(\mu)$ & funções somáveis com respeito a $\mu$ \\
\hline$\|x\|$ & norma de $x$ (em $\mathbb{R}^{n}$, norma euclidiana, salvo indicação contrária) \\
\hline$\mu\llcorner\mathrm{M}$ & restrição da medida exterior $\mu$ a $M$ \\
\hline$\left.\mu\right|_{\mathrm{M}}$ & traço da medida exterior $\mu \mathrm{em} \mathrm{M}$ \\
\hline$f_{\# \mu}$ & pushforward da medida exterior $\mu$ pela aplicação $f$ \\
\hline $\mathrm{D} f$ & derivada de Fréchet de $f$ \\
\hline$D_{f}$ & conjunto dos pontos em que $f$ é derivável \\
\hline$\llbracket L \rrbracket$ & jacobiano da aplicação linear $L$ \\
\hline J $f$ & a aplicação $x \in D_{f} \mapsto \llbracket \mathrm{D} f(x) \rrbracket$ \\
\hline$J_{f}^{0}$ & $\left\{x \in D_{f} \mid \exists \mathrm{J} f(x)=0\right\}$ \\
\hline$J_{f}^{+}$ & $\left\{x \in D_{f} \mid \exists \mathrm{J} f(x)>0\right\}$ \\
\hline$\Phi(m, n)$ & conjunto de todas as aplicações $\{1, \cdots, n\} \rightarrow\{1, \cdots, m\}$ \\
\hline$\Sigma(m, n)$ & é o conjunto $\{\lambda \in \Phi(m, n) \mid \lambda 1-1\}$ \\
\hline$\Lambda(m, n)$ & conjunto de funções estritamente crescentes $\{1, \cdots, n\} \rightarrow\{1, \cdots, m\}$ \\
\hline $\mathrm{L}^{1}(\mu)$ & é o conjunto de funções somáveis com respeito à medida $\mu$ \\
\hline$O(V, W)$ & conjunto de injeções ortogonais $V \rightarrow W$ \\
\hline $\operatorname{Sym}(n)$ & conjunto das aplicações lineares simétricas $\mathbb{R}^{n} \rightarrow \mathbb{R}^{n}$ \\
\hline
\end{tabular}


$\mu_{i} \stackrel{*}{\rightarrow} \nu \quad\left(\mu_{i}\right)$ é fraco-estrela convergente para $\nu$

$\Theta^{* n}(\mu, A, x)$ densidade superior $n$-dimensional de $A$ em $x$ com respeito a $\mu$

$\Theta_{*}^{n}(\mu, A, x) \quad$ densidade inferior $n$-dimensional de $A$ em $x$ com respeito a $\mu$

$\Theta^{n}(\mu, A, x) \quad$ densidade $n$-dimensional de $A$ em $x$ com respeito a $\mu$

$\operatorname{Lip}(f) \quad$ constante de Lipschitz de $f$

$\mu \ll \nu \quad \mu$ é absolutamente contínua em relação a $\nu$

$\mu \perp \nu \quad \mu$ e $\nu$ são mutuamente singulares

$\mathrm{C}_{c}^{0}\left(\mathbb{R}^{n}\right) \quad$ o espaço de funções continuas com suporte compacto em $\mathbb{R}^{n}$

$\mathrm{J}^{\mathrm{M}} f \quad$ jacobiano tangencial de $f$ em relação a $M$ 


\section{Lista de Figuras}

4.1 Os Blow-ups em $x_{0}$ da medida de Radon $\mathcal{H}^{1}\left\llcorner\Gamma\right.$ converge fraco estrela para $\mathcal{H}^{1}\llcorner\pi$. $\mathrm{Na}$ imagem temos que $0<r_{2}<r_{1}<1 \ldots \ldots \ldots \ldots$

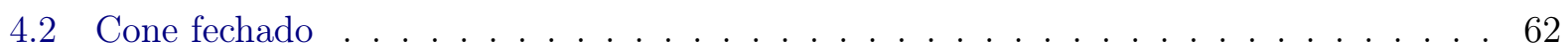




\section{Capítulo 1}

\section{Introdução}

A chamada teoria geométrica da medida é uma área da Matemática que se situa na fronteira entre a Análise e a Geometria. Grosso modo, pode-se dizer que o propósito desta teoria é investigar problemas geométricos através da teoria da medida. O nome "teoria geométrica da medida" deriva do tratado homônimo de H. Federer [9], publicado em 1969, o qual é até os dias atuais uma das principais referências da área.

A história da teoria geométrica da medida está atrelada à da teoria das superfícies mínimas e ao problema de Plateau ${ }^{1}$ no $\mathbb{R}^{3}$, i.e. o problema de encontrar uma superfície de menor área entre todas as superfícies cujo bordo seja uma dada curva suave, bem como a suas generalizações em dimensões/codimensões maiores. Uma das estratégias historicamente bem-sucedidas no ataque do referido problema é o chamado método direto do cálculo das variações; em particular, uma das etapas deste método envolve redefinir a noção clássica de superfície da Geometria Diferencial (na qual são considerados objetos que possuam regularidade $C^{1}$ ou maior) de modo a se considerar superfícies num sentido generalizado ou "fraco". Aqui, a ideia é similar à que se aplica na teoria das equações a derivadas parciais ao se considerar soluções num sentido fraco ou generalizado (dadas por funções de Sobolev, por exemplo); com a ampliação do espaço das possíveis soluções, ganham-se teoremas de compacidade que eventualmente garantam a existência de soluções de certos problemas de valores iniciais ou de contorno.

É com este espírito que, na teoria geométrica da medida, os objetos lisos da Geometria Diferencial clássica são substituídos por objetos com regularidade menor e que tenham propriedades similares sob o ponto de vista da teoria da medida. Assim, por exemplo, funções de classe $C^{1}$ ou maior são substituídas por funções Lipschitz (ou, mais geralmente, por funções aproximadamente diferenciáveis, cf. [9] cap. 3), e subvariedades mergulhadas de dimensão $k \mathrm{em} \mathbb{R}^{n}$ são substituídas por conjuntos $k$-retificáveis em $\mathbb{R}^{n}$. Estes são conjuntos que, do ponto de vista da teoria da medida, possuem propriedades tangenciais ou de primeira ordem similares às dos seus análogos lisos.

Em 1958, Federer publicou "Curvature Measures" ([8]), um artigo que deu os passos iniciais no sentido de se compreender, do ponto de vista da teoria da medida, as propriedades de segunda ordem de superfícies que carecem das propriedades de diferenciabilidade normalmente assumidas para discutir a curvatura. No mesmo artigo, também foi enunciada pela primeira vez e provada a chamada fórmula da coárea, uma poderosa generalização do clássico teorema de Fubini-Tonelli sobre integração com respeito a medidas produto. Federer menciona que se trata de um complemento à clássica fórmula da área ${ }^{2}$, e que ele a descobriu através da simplificação de alguns argumentos de Di Giorgi em [3]. A fórmula da área, por sua vez, é uma generalização do teorema de mudança de variáveis no contexto de integrais múltiplas em $\mathbb{R}^{n}$; embora seja mais antiga ${ }^{3}$, a versão para funções Lipschitz (ou, mais geralmente, para funções aproximadamente diferenciáveis) em subconjuntos retificáveis de espaços euclidianos é também devida a Federer, pelo que pudemos apurar.

\footnotetext{
${ }^{1}$ em homenagem a Joseph Plateau.

${ }^{2}$ em [8] Federer chama tais fórmulas genericamente de fórmulas integrais, mas a nomenclatura fórmula da área/coárea é usada em [9] e parece ter-se consolidado desde então.

${ }^{3}$ por exemplo, em 1933 C. B. Morrey já havia provado em [4] uma versão da fórmula da área para funções que ele chamou de "absolutamente contínuas no sentido de Tonelli".
} 
No presente trabalho, tem-se por objetivo investigar as fórmulas da área e da coárea para funções Lipschitz em subconjuntos retificáveis de $\mathbb{R}^{n}$, com provas mais acessíveis que as de [9] (que, embora seja a principal referência da área, é um texto difícil para iniciantes). Estas fórmulas são instrumentos importantíssimos em teoria geométrica da medida e generalizações das mesmas ainda se constituem em temas de pesquisa atual, a exemplo de [16], onde se provam versões fracas e fortes da fórmula da coárea e da regra da cadeia para determinantes jacobianos em espaços de Sobolev fracionais, e de [14], onde se prova uma versão da fórmula da coárea para funções em grupos Carnot de 2-etapas com estrutura sub-Lorentziana, além de muitas outras contribuições que aqui poderíamos citar.

Segue, abaixo, um breve resumo dos tópicos a serem abordados em cada capítulo.

No capítulo 2, "Preâmbulo", são recordadas algumas noções elementares da teoria da medida e integração e da teoria geométrica da medida que serão usadas ao longo deste trabalho.

No capítulo 3, "Fórmulas da Área e Coárea em Espaços Euclidianos", serão enunciadas e provadas versões das fórmulas da área e da coárea para aplicações Lipschitz $\mathbb{R}^{n} \rightarrow \mathbb{R}^{m}$, as quais serão usadas para provar os resultados mais gerais do capítulo 5 .

No capítulo 4, "Retificabilidade", introduziremos a noção de conjuntos k-retificáveis em $\mathbb{R}^{n}$. Estudaremos propriedades tangenciais desses conjuntos e faremos uma caracterização da noção de retificabilidade através de blow-ups de medidas de Radon (4.21).

Por fim, no capítulo 5, "Fórmulas da Área e Coárea em Conjuntos Retificáveis", serão enunciadas e provadas versões destas fórmulas para aplicações Lipschitz definidas em subconjuntos $k$-retificáveis de $\mathbb{R}^{n}$. Para tal, introduzimos a noção de diferenciabilidade tangencial em conjuntos retificáveis (veja 5.4), provamos uma versão do teorema de Rademacher para funções Lipschitz em conjuntos retificáveis (veja 5.6), e a seguir generalizamos as fórmulas da área e da coárea em espaços euclidianos para o contexto de funções Lipschitz em conjuntos retificáveis. 


\section{Capítulo 2}

\section{Preâmbulo}

Remetemos o leitor para [18] ou [6], se necessário, para noções elementares da teoria da medida e integração. Neste capítulo, fixaremos alguma notação e recordaremos algumas proposições/teoremas da referida teoria, os quais serão utilizados nos capítulos subsequentes. Enunciaremos também algumas proposições/teoremas iniciais em teoria geométrica da medida, cuja discussão foge do escopo deste texto, para os quais sugerimos as referências [12], [5], [9], [13] ou [7]. Para conveniência do leitor, indicaremos sugestões de referências junto a cada proposição ou teorema cuja demonstração for omitida. Os leitores mais experientes podem passar diretamente ao capítulo 3 e retornar aqui quando necessário.

\subsection{Medidas e Mensurabilidade}

Definição 2.1. Uma medida exterior ou, simplesmente, uma medida num conjunto $X$ é uma função $\mu: \mathscr{P}(X) \rightarrow[0, \infty]$ tal que:

1. $\mu(\emptyset)=0$;

2. (Monotonicidade) $\mu(A) \leq \mu(B)$ sempre que $A \subset B$;

3. (Subaditividade enumerável) $\mu\left(\cup_{n=1}^{\infty} A_{n}\right) \leq \sum_{n=1}^{\infty} \mu\left(A_{n}\right)$.

Observação 2.2. Nossa nomenclatura está de acordo com a comumente usada na Teoria Geométrica da Medida. No entanto, a maioria dos textos Análise Real (veja, por exemplo, [6]) reserva o termo "medida" para uma função definida em uma $\sigma$-álgebra $\mathcal{M}$ de subconjuntos de $X$, satisfazendo a aditividade enumerável, conforme definido abaixo em 2.6. Vamos usar o termo "medida" para ambos os tipos de funções de conjunto, se não houver risco de confusão; se o contexto não deixar claro, escreveremos explicitamente quando se trata de uma "medida exterior" ou de uma "medida numa $\sigma$-álgebra".

Definição 2.3. Dada uma medida exterior $\mu$ em um conjunto $X$, um subconjunto $A \subset X$ é chamado de mensurável em relação a $\mu$ (ou $\mu$-mensurável, ou, simplesmente, mensurável) se satisfizer a condição de Carathéodory: para todo $T \subset X$,

$$
\mu(T)=\mu(T \cap A)+\mu(T \backslash A) .
$$

Tendo em vista que a desigualdade " $\leq$ " na linha acima vale trivialmente, pela subaditividade enumerável, basta que se verifique a desigualdade oposta; e nesse caso, basta que se considere $T \subset X$ tal que $\mu(T)<\infty$, caso contrário a desigualdade oposta é trivial.

Denotamos por $\sigma(\mu)$ o conjunto de todos os subconjuntos mensuráveis de $X$ em relação a $\mu$.

Exemplo 2.4 (Medida de Lebesgue). Sejam $X=\mathbb{R}^{n}$ e $\mu: \mathscr{P}(X) \rightarrow[0, \infty]$ definida por $\mu(A):=$ $\inf \left\{\sum_{Q \in \mathcal{A}} \operatorname{vol}(Q) \mid \mathcal{A}\right.$ é uma cobertura enumerável de A por cubos com lados paralelos aos eixos coordenados $\}$, onde $\operatorname{vol}(Q)$ denota o volume euclidiano do cubo $Q$ (que não é assumido como 
aberto ou fechado, isto é, qualquer produto de intervalos com o mesmo comprimento é um cubo válido). Então $\mu$ é uma medida em $\mathbb{R}^{n}$, chamada de medida de Lebesgue. Denotamos a medida de Lebesgue em $\mathbb{R}^{n}$ por $|\cdot|$ ou $\mathcal{L}^{n}$. Interpretamos a medida de Lebesgue $\mathcal{L}^{n}(A)$ como o "volume euclidiano $n$-dimensional de $A^{\prime \prime}$.

Denotamos o conjunto $\sigma\left(\mathcal{L}^{n}\right)$ de conjuntos Lebesgue mensuráveis por $\mathscr{L}_{\mathbb{R}^{n}}$ (ou, simplesmente, $\mathscr{L})$.

Observação 2.5. 1) $\mathcal{L}^{n}$ é invariante por translações, isto é, para todo $x \in \mathbb{R}^{n}$ e para todo $A \subset \mathbb{R}^{n}$, $\mathcal{L}^{n}(A+x)=\mathcal{L}^{n}(A)$.

2) $\mathcal{L}^{n}$ é homogêneo de grau $n$ com respeito a homotetias, isto é, para todo $\lambda>0$ e para todo $A \subset \mathbb{R}^{n}, \mathcal{L}^{n}(\lambda A)=\lambda^{n} \mathcal{L}^{n}(A)$.

Definição 2.6. Dado $X$ um conjunto, $\mathcal{M} \subset \mathscr{P}(X)$ é chamado de álgebra de subconjuntos de $X$ se contiver o conjunto vazio, for fechado por complementação e por uniões finitas. $\mathcal{M}$ é chamado de $\sigma$-álgebra se for uma álgebra fechada por uniões enumeráveis. Os conjuntos em $\mathcal{M}$ são chamados mensuráveis com respeito a $\mathcal{M}$, ou $\mathcal{M}$-mensuráveis, ou (se ficar claro pelo contexto), simplesmente, mensuráveis.

Dada uma $\sigma$-álgebra $\mathcal{M} \subset \mathscr{P}(X)$, chamamos uma função $\mu: \mathcal{M} \rightarrow[0, \infty]$ de medida em $\mathcal{M}$ se satisfizer:

1. $\mu(\emptyset)=0$;

2. (aditividade enumerável) para toda sequência disjunta $\left(A_{n}\right)_{n \in \mathbb{N}}$ em $\mathcal{M}, \mu\left(\cup_{n=1}^{\infty} A_{n}\right)=\sum_{n=1}^{\infty} \mu\left(A_{n}\right)$.

Uma medida $\mu: \mathcal{M} \rightarrow[0, \infty]$ é chamada:

- completa se $\forall E \in \mathcal{M}, \mu(E)=0$ e $A \subset E$ implica $A \in \mathcal{M}$;

- finita se $\mu(X)<\infty$;

- $\sigma$-finita se existe uma sequência $\left(E_{n}\right)_{n \in \mathbb{N}}$ em $\mathcal{M}$ tal que $\cup_{n \in \mathbb{N}} E_{n}=X$ e, para todo $n \in \mathbb{N}$, $\mu\left(E_{n}\right)<\infty$. Mais geralmente, um conjunto $A \subset X$ diz-se $\sigma$-finito se puder ser coberto por uma quantidade enumerável de conjuntos mensuráveis de medidas finitas.

Teorema 2.7 (de Carathéodory, [6], [9]). Seja $\mu$ uma medida exterior num conjunto X. Então $\sigma(\mu)$ é uma $\sigma$-álgebra de subconjuntos de $X$ e a restrição de $\mu$ a $\sigma(\mu)$ é uma medida completa.

Definição 2.8. Uma medida exterior $\mu: \mathscr{P}(X) \rightarrow[0, \infty]$ é chamada:

- regular se, para todo $A \subset X$, existe $E \in \sigma(\mu)$ tal que $A \subset E$ e $\mu(A)=\mu(E)$;

- finita ou $\sigma$-finita se assim for sua restrição a $\sigma(\mu)$.

Dado $A \subset X$, não necessariamente $\mu$-mensurável, diz-se que $A$ é $\sigma$-finito com respeito a $\mu$ se puder ser escrito como união enumerável de conjuntos (não necessariamente mensuráveis) de medidas finitas.

Proposição 2.9 (Propriedades de continuidade das medidas, [6], [9], [12]). Para uma medida exterior $\mu$ em $X$, as seguintes propriedades são válidas:

i) (continuidade para cima) se $\left(E_{n}\right)_{n \in \mathbb{N}}$ é uma sequência crescente em $\sigma(\mu)$, então

$$
\mu\left(\cup_{n=1}^{\infty} E_{n}\right)=\lim _{n \rightarrow \infty} \mu\left(E_{n}\right)
$$

ii) (continuidade para baixo) se $\left(E_{n}\right)_{n \in \mathbb{N}}$ é uma sequência decrescente em $\sigma(\mu)$ e $\mu\left(E_{1}\right)<\infty$, então

$$
\mu\left(\cap_{n=1}^{\infty} E_{n}\right)=\lim _{n \rightarrow \infty} \mu\left(E_{n}\right) .
$$

Observação 2.10 ([9]). Com a notação da proposição anterior, se $\mu$ for regular, a propriedade i) vale para toda sequência crescente $\left(E_{n}\right)_{n \in \mathbb{N}}$ de subconjuntos de $X$ (não necessariamente mensuráveis). 


\subsubsection{Envoltórios}

Definição 2.11 (envoltório). Sejam $\mu$ uma medida num conjunto $X$ e $A \subset X$. Diz-se que $B \in \sigma(\mu)$ é um $\mu$-envoltório de $A$ se $A \subset B$ e, para todo $T \in \sigma(\mu), \mu(T \cap A)=\mu(T \cap B)$.

Proposição 2.12 (existência de envoltórios). Sejam $\mu$ uma medida num conjunto $X e A \subset X$.

i) Se $B \in \sigma(\mu)$ é tal que $A \subset B$ e $\mu(A)=\mu(B)<\infty$, então $B$ é um $\mu$-envoltório de $A$.

ii) Se $\mu$ for regular e A for $\sigma$-finito com respeito a $\mu$, então $A$ admite um $\mu$-envoltório.

Demonstração. $\quad$ i) Seja $T \in \sigma(\mu)$. Tem-se: $\mu(T \cap B)=\mu(B)-\mu(B \backslash T) \leq \mu(A)-\mu(A \backslash T)=$ $\mu(T \cap A) \leq \mu(T \cap B), \log 0 \mu(T \cap B)=\mu(T \cap A)$.

ii) O caso em que $\mu(A)<\infty$ segue imediatamente do item anterior, pois a regularidade de $\mu$ garante a existência de $B \in \sigma(\mu)$ tal que $A \subset B$ e $\mu(A)=\mu(B)<\infty$.

No caso geral, pela $\sigma$-finitude de $A$ com respeito a $\mu$, podemos tomar $\left(A_{n}\right)_{n \in \mathbb{N}}$ sequência crescente de subconjuntos de $X$ tal que $\cup_{n \in \mathbb{N}} A_{n}=A$ e, $\forall n \in \mathbb{N}, \mu\left(A_{n}\right)<\infty$. Pelo caso já provado, para todo $n \in \mathbb{N}$, existe $B_{n} \in \sigma(\mu)$ envoltório de $A_{n}$ com respeito a $\mu$. Por indução sobre $n$ é imediato verificar que, para cada $n \in \mathbb{N}, \cup_{i=1}^{n} B_{i}$ também é envoltório de $A_{n}$ com respeito a $\mu$; assim, substituindo-se $B_{n}$ por $\cup_{i=1}^{n} B_{i}$, se necessário, podemos supor que a sequência $\left(B_{n}\right)_{n \in \mathbb{N}}$ é crescente.

Note que as sequências $\left(T \cap A_{n}\right)_{n \in \mathbb{N}}$ e $\left(T \cap B_{n}\right)_{n \in \mathbb{N}}$ crescem, respectivamente, para $T \cap A$ e $T \cap \cup_{n \in \mathbb{N}} B_{n}$. Daí, para todo $T \in \sigma(\mu)$, como $\mu$ é regular, segue da observação 2.10 que

$$
\mu(T \cap A)=\lim _{n \rightarrow \infty} \mu\left(T \cap A_{n}\right)=\lim _{n \rightarrow \infty} \mu\left(T \cap B_{n}\right)=\mu\left(T \cap \cup_{n \in \mathbb{N}} B_{n}\right) .
$$

Pela arbitrariedade do $T \in \sigma(\mu)$ tomado, isso mostra que $\cup_{n \in \mathbb{N}} B_{n} \in \sigma(\mu)$ é um envoltório de $A$, o que conclui a demonstração.

\subsubsection{Operações com Medidas}

Três maneiras úteis de se construir medidas a partir de medidas dadas são as restrições, os traços e o pushforward.

Definição 2.13 (restrições e traços de medidas). Seja $\mu$ uma medida exterior em $X$ e $A \subset X$. Definimos:

1. a restrição de $\mu$ a $A$, denotada por $\mu\llcorner A$, como a medida $\mathscr{P}(X) \rightarrow[0, \infty]$ dada por $E \rightarrow$ $\mu(A \cap E)$;

2. o traço de $\mu$ em $A$, denotado por $\left.\mu\right|_{A}$, como a medida $\mathscr{P}(A) \rightarrow[0, \infty]$ dada por $E \rightarrow \mu(E)$, i.e. a restrição a $\mathscr{P}(A) \subset \mathscr{P}(X)$ da função $\mu: \mathscr{P}(X) \rightarrow[0 . \infty]$.

Note que a restrição $\mu\left\llcorner A\right.$ é uma medida em $X$, enquanto que o traço $\left.\mu\right|_{A}$ é uma medida em $A$. Além disso, não assumimos que $A$ seja $\mu$-mensurável.

Definição 2.14 (pushforward de medidas). Sejam $\mu$ uma medida em $X$ e $f: X \rightarrow Y$ uma aplicação a valores no conjunto $Y$. Definimos uma medida $\mathscr{P}(Y) \rightarrow[0, \infty]$ em $Y$ por:

$$
A \subset Y \rightarrow \mu\left(f^{-1}(A)\right),
$$

a qual é chamada de pushforward de $\mu$ por $f$ e denotada por $f_{\#} \mu$.

Proposição 2.15 ([7]). Sejam $\mu$ uma medida no conjunto $X, A \subset X$ e $f: X \rightarrow Y$. Valem as seguintes propriedades: 
i) $\sigma(\mu) \subset \sigma(\mu\llcorner A)$.

ii) Se $E \in \sigma(\mu)$, então $E \cap A \in \sigma\left(\left.\mu\right|_{A}\right)$. Além disso, se $A \in \sigma(\mu)$, então $\sigma\left(\left.\mu\right|_{A}\right)=\sigma(\mu) \cap 2^{A}=$ $\{E \in \sigma(\mu) \mid E \subset A\}$.

iii) Se $B \subset Y, f^{-1}(B)$ é $\mu$-mensurável se, e somente se, $\forall A \subset X, B$ é $f_{\#}(\mu\llcorner A)$-mensurável.

\subsubsection{Medidas em Espaços Topológicos}

Acrescentemos, agora, uma topologia $\tau$ no conjunto $X$. Devemos considerar medidas em $X$ que interajam com a topologia, no sentido de ter boas propriedades de regularidade e aproximação, conforme será exposto abaixo.

Dado um subconjunto $S \subset \mathscr{P}(X)$, existe uma menor $\sigma$-álgebra de subconjuntos de $X$ que contém $S$, a saber, a interseção da família de todas as $\sigma$-álgebras em $X$ que contêm $S$ (esta família não é vazia, uma vez que $\mathscr{P}(X)$ é uma tal $\sigma$-álgebra). Denotamos esta $\sigma$-álgebra por $\sigma(S)$, a chamada $\sigma$-álgebra gerada por $S$.

Definição 2.16. Num espaço topológico $(X, \tau)$, definimos a $\sigma$-álgebra de Borel como a $\sigma$-álgebra gerada por $\tau$, ou seja, $\sigma(\tau)$. Notação: $\mathscr{B}_{X}$ ou $\mathscr{B}(X)$. Os elementos de $\mathscr{B}_{X}$ são chamados conjuntos de Borel ou borelianos.

Uma medida $\mu$ em $X$ é uma medida de Borel se cada conjunto de Borel é $\mu$-mensurável, ou seja, se $\mathscr{B}_{X} \subset \sigma(\mu)$. Uma medida Borel-regular em $X$ é uma medida de Borel em $X$ que satisfaz: para todo $A \subset X$, existe $E \in \mathscr{B}_{X}$ tal que $A \subset E$ e $\mu(A)=\mu(E)$.

O seguinte teorema fornece um critério importante para que uma medida num espaço métrico seja boreliana.

Teorema 2.17 (critério de Carathéodory, [12], [9]). Uma medida $\mu$ num espaço métrico $(X, d)$ é boreliana se, e somente se,

$$
\mu(A \cup B)=\mu(A)+\mu(B)
$$

sempre que $A, B \subset X$ e d $(A, B):=\inf \{d(a, b) \mid a \in A, b \in B\}>0$.

Definição 2.18. Uma medida de Borel $\mu$ em um espaço topológico $(X, \tau)$ é chamada:

1. aberta $\sigma$-finita se existe uma sequência $\left(U_{n}\right)_{n \in \mathbb{N}}$ de subconjuntos abertos de $X$ tal que $X=$ $\cup_{n \in \mathbb{N}} U_{n}$ e para todo $n \in \mathbb{N}, \mu\left(U_{n}\right)<\infty$.

2. localmente finita se, para cada $x \in X$, existe uma vizinhança aberta $U$ de $x$ tal que $\mu(U)<\infty$.

Proposição 2.19 (envoltórios borelianos). Sejam $\mu$ uma medida Borel-regular num espaço topológico $X$ e $A \subset X$ um conjunto (não necessariamente mensurável) $\sigma$-finito com respeito a $\mu$. Então $A$ admite um $\mu$-envoltório boreliano $B$, i.e. existe $B \in \mathscr{B}_{X}$ tal que $B$ é $\mu$-envoltório de $A$.

Demonstração. Se $\mu(A)<\infty$, a Borel-regularidade de $\mu$ garante a existência de $B \in \mathscr{B}_{X}$ tal que $A \subset B$ e $\mu(A)=\mu(B)<\infty$; daí, segue da prop. 2.12.i) que $B$ é $\mu$-envoltório de $A$.

No caso geral, a $\sigma$-finitude de $A$ com respeito a $\mu$ permite escrever $A=\cup_{n \in \mathbb{N}} A_{n} \operatorname{com}\left(A_{n}\right)_{n \in \mathbb{N}}$ crescente e, para todo $n \in \mathbb{N}, \mu\left(A_{n}\right)<\infty$. Pelo caso já provado, para cada $n \in \mathbb{N}$, podemos tomar $B_{n} \subset X$ envoltório boreliano de $A_{n}$. Então, pelo mesmo argumento da prova da prop. 2.12.ii), conclui-se que $\cup_{n \in \mathbb{N}} B_{n} \in \mathscr{B}_{X}$ é $\mu$-envoltório de $A$.

Corolário 2.20. Sejam $\mu$ uma medida Borel-regular num espaço topológico $X$ e $A \subset X$ um conjunto $\mu$-mensurável e $\sigma$-finito. Então existem borelianos $B, D \subset X$ tais que $D \subset A \subset B$ e $\mu(B \backslash D)=0$.

Demonstração. Tome $B \supset A$ envoltório boreliano de $A$. Como $A \in \sigma(\mu)$, tem-se $B \backslash A \in \sigma(\mu) ; \operatorname{logo}$, pela definição de $\mu$-envoltório, tem-se $\mu(B \backslash A)=\mu((B \backslash A) \cap B)=\mu((B \backslash A) \cap A)=\mu(\emptyset)=0$. Em particular, existe $E \supset B \backslash A$ envoltório boreliano de $B \backslash A$, de modo que $\mu(E)=0$. Defina $D:=B \backslash E \in \mathscr{B}_{X}$. Então $D \subset A \subset B$ e $B \backslash D=E \cap B \subset E$, logo $\mu(B \backslash D)=0$. 
Teorema 2.21 (aproximação por abertos e fechados, [9], [7]). Seja $\mu$ uma medida Borel-regular aberta $\sigma$-finita num espaço topológico $(X, \tau)$ no qual todo fechado é um $G_{\delta}$ (i.e. uma intersecção enumerável de abertos). Valem as seguintes propriedades de aproximação:

i) (aproximação por fora por abertos) $\forall A \subset X, \mu(A)=\inf \{\mu(U) \mid A \subset U \in \tau\}$;

ii) (aproximação por dentro por fechados) $\forall A \in \sigma(\mu), \mu(A)=\sup \{\mu(C) \mid C \subset A, C$ fechado $\}$.

Observação 2.22. O teorema anterior vale, em particular, para uma medida Borel-regular localmente finita num espaço métrico separável.

Definição 2.23. Uma medida de Radon num espaço topológico Hausdorff localmente compacto $(X, \tau)$ é uma medida de Borel $\mu$ em $X$ tal que:

i) (finitude sobre conjuntos compactos) Se $K$ é um subconjunto compacto de $X$, então $\mu(K)<\infty$.

ii) (Regularidade interior nos conjuntos abertos) Para todo $U \subset X$ aberto,

$$
\mu(U)=\sup \{\mu(K) \mid K \subset U, K \text { compacto }\} .
$$

iii) (Regularidade exterior) Para todo $A \subset X$,

$$
\mu(A)=\inf \{\mu(U) \mid A \subset U, \text { Uaberto }\} .
$$

Observação 2.24 .

1) Se $\mu$ é uma medida de Radon num espaço topológico Hausdorff localmente compacto $(X, \tau)$, então $\mu$ é regular internamente em todos os conjuntos $\mu$-mensuráveis $\sigma$-finitos, i.e. a propriedade 2.23(ii) vale para qualquer $A$ conjunto $\mu$-mensurável $\sigma$-finito no lugar de $U$. Em particular, se $\mu$ é $\sigma$-finita, a propriedade 2.23(ii) vale para todos os conjuntos $\mu$-mensuráveis.

2) Num espaço métrico localmente compacto separável $X$, uma medida é de Radon se, e somente se, for Borel-regular e localmente finita.

Definição 2.25 (Suporte de uma medida num espaço topológico). Seja $\mu$ uma medida num espaço topológico $X$.

1. Dizemos que $\mu$ é concentrada sobre um conjunto $A \subset X$, se $\mu(X \backslash A)=0$.

2. O suporte de $\mu$, denotado por spt $\mu$, é o complemento da união de todos os conjuntos abertos $V \subset X$ tal que $\mu(V)=0$.

Proposição 2.26 ([7]). Sejam $\mu$ uma medida sobre um conjunto $X$ e $A \subset X$. As seguintes propriedades são válidas:

i) Se $X$ é um espaço métrico, $\mu$ é uma medida Borel-regular em $X$ e 1) $A \in \mathscr{B}_{X}$ ou 2) $A \in \sigma(\mu)$ e $\mu(A)<\infty$, então $\mu\llcorner A$ é Borel regular.

ii) Se $X$ é um espaço métrico separável localmente compacto, $\mu$ uma medida de Radon em $X$ e 1) $A \in \mathscr{B}_{X}$ ou 2) $A \in \sigma(\mu)$ e $\mu(A)<\infty$, então $\mu\llcorner A$ é uma medida de Radon.

Proposição 2.27 ([9], [7]). Sejam $X$ e $Y$ espaços métricos separáveis localmente compactos, $f$ : $X \rightarrow Y$ uma aplicação contínua e própria, e $\mu$ uma medida de Radon em $X$. Então $f_{\#} \mu$ é uma medida de Radon em $Y$ e spt $f_{\#} \mu=f(\operatorname{spt} \mu)$. 


\subsubsection{Aplicações Mensuráveis}

Definição 2.28 (Espaços mensuráveis e aplicações mensuráveis). Um espaço mensurável é um par $(X, \mathcal{M})$ onde $X$ é um conjunto e $\mathcal{M}$ é uma $\sigma$-álgebra de subconjuntos de $X$. Os elementos de $\mathcal{M}$ são chamados de $\mathcal{M}$-mensuráveis (ou, simplesmente, mensuráveis). Dados os espaços mensuráveis $(X, \mathcal{M})$ e $(Y, \mathcal{N})$, uma aplicação $f: X \rightarrow Y$ é chamada de mensurável com respeito a $\mathcal{M}$ e $\mathcal{N}$ (ou simplesmente mensurável) se, para todo $A \in \mathcal{N}, f^{-1}(A) \in \mathcal{M}$.

Se $X$ (ou $Y$ ) é um espaço topológico, assumiremos tacitamente que a $\sigma$-álgebra $\mathcal{M}$ é a $\sigma$-álgebra $\mathscr{B}_{X}$ de Borel, a menos que outra $\sigma$-álgebra seja explicitamente especificada.

Definição 2.29 (Aplicações $\mu$-mensuráveis). Sejam $\mu$ uma medida em $X$ e $Y$ um espaço topológico. Uma função $f$ : dom $f \subset X \rightarrow Y$ é chamada de mensurável com respeito a $\mu$ (ou $\mu$-mensurável) se as seguintes condições forem satisfeitas:

1. Seu domínio cobre quase todo $X$, ou seja, $\mu(X \backslash \operatorname{dom} f)=0$.

2. Para todo $B \in \mathscr{B}_{Y}, f^{-1}(B)$ é $\mu$-mensurável.

Observação 2.30. 1) Se $f$ é $\mu$-mensurável, no sentido da definição anterior, qualquer outra função que coincida com $f$, exceto para um conjunto de medida nula, também é $\mu$-mensurável.

2) Dizer que $f$ é $\mu$-mensurável, no sentido da definição anterior, é equivalente a dizer que $f$ admite uma extensão $X \rightarrow Y$ que seja mensurável com respeito a $\sigma(\mu)$ e $\mathscr{B}_{Y}$, no sentido da definição 2.28 .

Listamos abaixo algumas das principais propriedades das aplicações mensuráveis.

Teorema 2.31 (Propriedades de aplicações mensuráveis, [6], [9]). Sejam $(X, \mathcal{M}),(Y, \mathcal{N}),(Z, \mathcal{O})$ espaços mensuráveis. Valem as seguintes propriedades:

i) $f: X \rightarrow Y$ é mensurável se, e somente se, dado $S \subset \mathscr{P}(Y)$ tal que $\sigma(S)=\mathcal{N}$, para todo $B \in S, f^{-1}(B) \in \mathcal{M}$.

ii) Se $f: X \rightarrow Y$ e $g: Y \rightarrow Z$ são mensuráveis, $g \circ f$ também o é.

iii) Se $X$ e $Y$ são espaços topológicos e $f: X \rightarrow Y$ é contínua, então $f$ é boreliana.

iv) $S e\left(f_{n}\right)_{n \in \mathbb{N}}$ é uma sequência de aplicações mensuráveis $X \rightarrow \overline{\mathbb{R}}$, as seguintes aplicações $X \rightarrow$

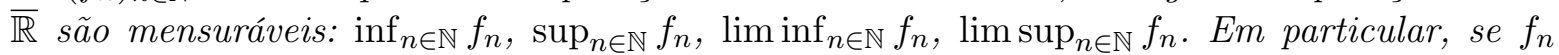
converge pontualmente, a função limite é mensurável. Mais geralmente, se $Y$ é um espaço métrico e $\left(f_{n}\right)_{n \in \mathbb{N}}$ é uma sequência pontualmente convergente de funções mensuráveis $X \rightarrow Y$, o limite da sequência é mensurável.

Corolário 2.32. Se $f, g: X \rightarrow \overline{\mathbb{R}}$ são ambas mensuráveis, então $\max \{f, g\}$ e $\min \{f, g\}$ também o são. Em particular, as partes positiva $f^{+}:=\max \{f, 0\}$ e negativa $f^{-}:=\max \{-f, 0\}$ de $f$ são mensuráveis.

Notação. Seja $(X, \mathcal{M})$ espaço mensurável. Dado $A \subset X$, denotamos por $\chi_{A}$ a função característica ou indicatriz de $A$, i.e. a função $X \rightarrow \mathbb{R}$ definida por $\chi_{A}(x)=1$ se $x \in A$ e $\chi_{A}(x)=0$ se $x \notin A$. Note que $\chi_{A}$ é mensurável se, e somente se, $A \in \mathcal{M}$.

Proposição 2.33. Sejam $(X, \mathcal{M})$ espaço mensurável, $f: X \rightarrow[0, \infty]$ mensurável, $\left(r_{n}\right)_{n \in \mathbb{N}}$ sequência em $(0, \infty)$ tal que $r_{n} \rightarrow 0$ e $\sum_{n=1}^{\infty} r_{n}=\infty$. Então existe uma sequência $\left(A_{n}\right)_{n \in \mathbb{N}}$ em $\mathcal{M}$ tal que $\sum_{k=1}^{n} r_{k} \chi_{A_{k}}$ cresce pontualmente para $f$. 
Demonstração. Definamos $\left(A_{k}\right)_{n \in \mathbb{N}}$ e $\left(g_{k}\right)_{k \in \mathbb{N}}$ indutivamente por

$$
\begin{aligned}
A_{1}:=\left\{x \in X \mid r_{1} \leq f(x)\right\} & \text { e } \quad g_{1}:=r_{1} \chi_{A_{1}} \\
& \vdots \\
A_{k}:=\left\{x \in X \mid g_{k-1}(x)+r_{k} \leq f(x)\right\} & \text { e } \quad g_{k}:=g_{k-1}+r_{k} \chi A_{k} .
\end{aligned}
$$

A sequência $\left(A_{n}\right)_{n \in \mathbb{N}}$ assim definida está em $\mathcal{M}$ e é tal que $\sum_{k=1}^{n} r_{k} \chi_{A_{k}}$ cresce pontualmente para $f$.

O seguinte é um critério útil para se testar a mensurabilidade em termos de coberturas mensuráveis enumeráveis.

Proposição 2.34. Sejam $(X, \mathcal{M}),(Y, \mathcal{N})$ espaços mensuráveis e $\left(A_{n}\right)_{n \in \mathbb{N}}$ uma sequência em $\mathcal{M}$ tal que $\cup_{n \in \mathbb{N}} A_{n}=X$. Então uma aplicação $f: X \rightarrow Y$ é mensurável se, e somente se, $\forall n \in \mathbb{N}$, $\left.f\right|_{A_{n}}: A_{n} \rightarrow Y$ é mensurável, onde cada $A_{n}$ é munido da $\sigma$-álgebra traço $\left.\mathcal{M}\right|_{A_{n}}:=\left\{E \cap A_{n} \mid E \in\right.$ $\mathcal{M}\}$.

Demonstração. Para cada $B \in \mathcal{N}, f^{-1}(B)=\left.\cup_{n \in \mathbb{N}} f\right|_{A_{n}} ^{-1}(B) \in \mathcal{M}$, pois, para todo $n \in \mathbb{N},\left.f\right|_{A_{n}} ^{-1}(B) \in$ $\left.\mathcal{M}\right|_{A_{n}} \subset \mathcal{M}$.

\subsection{Teoria de Integração}

Fixemos uma uma medida $\mu$ num conjunto $X$. Usaremos os fatos, notações e nomenclatura usuais da teoria de integração no espaço de medida $(X, \sigma(\mu), \mu)$; para os leitores que julgarem necessário, sugerimos as referências [6], [18] ou outros textos clássicos em Análise Real.

Definição 2.35 (integrabilidade e somabilidade). Adotamos a seguinte nomenclatura: dada uma função $\mu$-mensurável $f: X \rightarrow \overline{\mathbb{R}}$, diz-se que $f$ é

- integrável se $\int f^{+} \mathrm{d} \mu<\infty$ ou $\int f^{-} \mathrm{d} \mu<\infty$; caso afirmativo, definimos

$$
\int f \mathrm{~d} \mu:=\int f^{+} \mathrm{d} \mu-\int f^{-} \mathrm{d} \mu \in \overline{\mathbb{R}} .
$$

- somável se pertencer a $\mathrm{L}^{1}(\mu)$, i.e. se $\int f^{+} \mathrm{d} \mu<\infty$ e $\int f^{-} \mathrm{d} \mu<\infty$ (ou, equivalentemente, se $\left.\int|f| \mathrm{d} \mu<\infty\right)$.

Em particular, note que, se $f$ for $\mu$ mensurável não negativa, então $f$ é integrável (pois $f^{-} \equiv 0$ ).

Abaixo recordaremos, para uso posterior, as noções de integral superior e inferior de uma função não negativa (não necessariamente mensurável) em $X$, e a construção de produtos de medidas exteriores.

\subsubsection{Integrais Superior e Inferior}

Nesta subseção, introduziremos as noções de integral superior e inferior de uma função não negativa $f$ em $X$, e as relacionaremos com a integral de $f$ no caso em que $f$ seja mensurável. Remetemos o leitor para [9], seção 2.4, caso necessite de mais detalhes.

Definição 2.36. Seja $f: X \rightarrow[0, \infty]$, não necessariamente $\mu$-mensurável. Definimos:

1) a integral superior de $f$, denotada por $\int^{*} f \mathrm{~d} \mu$ ou $\int^{*} f$ caso a medida fique subentendida, por

$$
\inf \left\{\int \varphi \mathrm{d} \mu \mid \varphi: X \rightarrow[0, \infty) \text { mensurável, } \operatorname{Im} \varphi \text { enumerável, } \varphi \geq f \mu \text {-q.s. }\right\} ;
$$


2) a integral inferior de $f$, denotada por $\int_{*} f \mathrm{~d} \mu$ ou $\int_{*} f$ caso a medida fique subentendida, por

$$
\sup \left\{\int \varphi \mathrm{d} \mu \mid \varphi: X \rightarrow[0, \infty) \text { mensurável, } \operatorname{Im} \varphi \text { enumerável, } \varphi \leq f \mu \text {-q.s. }\right\} \text {. }
$$

Proposição 2.37 ([9], [7]). Com a notação da definição acima, dada $f: X \rightarrow[0, \infty]$, valem as seguintes propriedades:

i) Se $\int_{*} f=\int^{*} f<\infty$, então $f$ é $\mu$-mensurável e $\int f$ coincide com a integral superior e inferior; em particular, $f \in \mathrm{L}^{1}(\mu)$.

ii) Se $f$ for $\mu$-mensurável, então $\int_{*} f=\int^{*} f=\int f$.

iii) O teorema da convergência monótona vale para a integral superior, i.e. se $\left(f_{n}\right)_{n \in \mathbb{N}}$ for uma sequência de funções não negativas (não necessariamente mensuráveis) que cresça $\mu$-q.s. para uma função $f$ (não necessariamente mensurável), então $\int^{*} f_{n} \rightarrow \int^{*} f$. Analogamente, o lema de Fatou também vale para a integral superior.

iv) $S e\left(f_{n}\right)_{n \in \mathbb{N}}$ for uma sequência de funções não negativas (não necessariamente mensuráveis) tal que $\int^{*} f_{n} \rightarrow 0$, existe uma subsequência de $\left(f_{n}\right)_{n \in \mathbb{N}}$ que converge $\mu$-q.s. para zero.

v) Seja $\left(f_{n}\right)_{n \in \mathbb{N}}$ uma sequência de funções $X \rightarrow[0, \infty]$. Então

$$
\begin{aligned}
& \int_{*} \sum_{n=1}^{\infty} f_{n} \mathrm{~d} \mu \geq \sum_{n=1}^{\infty} \int_{*} f_{n} \mathrm{~d} \mu, \\
& \int^{*} \sum_{n=1}^{\infty} f_{n} \mathrm{~d} \mu \leq \sum_{n=1}^{\infty} \int^{*} f_{n} \mathrm{~d} \mu .
\end{aligned}
$$

vi) Dada $f: X \rightarrow[0, \infty]$, seja $\psi: 2^{X} \rightarrow[0, \infty]$ definida por $\psi(A):=\int_{A}^{*} f \mathrm{~d} \mu:=\int^{*} \chi_{A} f \mathrm{~d} \mu$. Então $\psi$ é uma medida em $X$ e $\sigma(\mu) \subset \sigma(\psi)$. Além disso, $\psi(A)=0$ sempre que $\mu(A)=0$, i.e. $\psi$ é absolutamente contínua com respeito a $\mu$. Finalmente, se $\mu$ é $\sigma$-finita, $f \mu$-mensurável e: 1) se $\mu$ é regular, $\psi$ também o é; 2) se $X$ é um espaço topológico e $\mu$ é Borel-regular, $\psi$ também o é.

\subsubsection{Medidas Produto e Teorema de Fubini-Tonelli}

Em adição à medida $\mu$ no conjunto $X$, fixaremos nesta subseção uma medida $\nu$ num segundo espaço $Y$. Introduziremos abaixo a noção de medida (exterior) produto das medidas $\mu$ e $\nu$. Recorde que, conforme a convenção aritmética estendida usualmente adotada em Medida e Integração,

$$
0 \cdot \infty=0
$$

Definição 2.38 (produtos de medidas exteriores). Definimos, para todo $E \subset X \times Y, \mu \times \nu(E):=$ $\inf \left\{\sum_{n \in \mathbb{N}} \mu\left(A_{n}\right) \nu\left(B_{n}\right) \mid \forall n \in \mathbb{N}, A_{n} \in \sigma(\mu), B_{n} \in \sigma(\nu), E \subset \cup_{n \in \mathbb{N}} A_{n} \times B_{n}\right\} \in[0, \infty]$. Chamamos $\mu \times \nu$ de medida produto de $\mu$ e $\nu$.

Definimos de forma análoga o produto de qualquer número finito de medidas exteriores.

O teorema abaixo, que pode ser obtido como consequência direta do teorema de Fubini-Tonelli para medidas em $\sigma$-álgebras, garante que $\mu \times \nu$ definida acima é, de fato, uma medida exterior. Note que $\mu \times \nu$ é sempre uma medida regular, mas não assumimos a regularidade de $\mu$ ou $\nu$.Fixamos a seguinte:

Notação (seções).

- Para $E \subset X \times Y$ e $\left(x_{0}, y_{0}\right) \in X \times Y, E_{x_{0}}:=\left\{y \in Y \mid\left(x_{0}, y\right) \in E\right\}$ (a $x_{0}$-seção de $E$ ) e $E_{y_{0}}:=\left\{x \in X \mid\left(x, y_{0}\right) \in E\right\}\left(\right.$ a $y_{0}$-seção of $\left.E\right)$. 
- Para uma função $f$ definida em dom $f \subset X \times Y$ e $\left(x_{0}, y_{0}\right) \in X \times Y, f_{x_{0}}$ (a $x_{0}$-seção of $f$ ) e $f_{y_{0}}\left(\right.$ a $y_{0}$-seção de $\left.f\right)$ são as funções definidas, respectivamente, em $(\operatorname{dom} f)_{x_{0}}$ e $(\operatorname{dom} f)_{y_{0}}$ por $y \mapsto f\left(x_{0}, y\right)$ e $x \mapsto f\left(x, y_{0}\right)$.

Teorema 2.39 (teorema de Fubini-Tonelli para produtos de medidas exteriores, [9], [12]). Com a notação da definição anterior, $\mu \times \nu: 2^{X \times Y} \rightarrow[0, \infty]$ é uma medida regular. Além disso:

i) $S e A \in \sigma(\mu)$ e $B \in \sigma(\nu)$, então $A \times B \in \sigma(\mu \times \nu)$ e $\mu \times \nu(A \times B)=\mu(A) \nu(B)$.

ii) Se $E \in \sigma(\mu \times \nu)$ é $\sigma$-finito com respeito a $\mu \times \nu$, então, para $\mu$-quase todo $x \in X, E_{x} \in \sigma(\nu)$, e para $\nu$-quase todo $y \in Y, E_{y} \in \sigma(\mu)$. As funções $x \mapsto \nu\left(E_{x}\right)$ e $y \mapsto \mu\left(E_{y}\right)$ são mensuráveis, e a medida de $E$ pode ser calculada por:

$$
\mu \times \nu(E)=\int \nu\left(E_{x}\right) \mathrm{d} \mu(x)=\int \mu\left(E_{y}\right) \mathrm{d} \nu(y) .
$$

iii) Se $f$ é uma função integrável definida em $\operatorname{dom} f \subset X \times Y$ tal que $\{f \neq 0\}$ é $\sigma$-finito com respeito a $\mu \times \nu$ (o que vale, em particular, se $f$ for somável), então, para $\mu$-quase todo $x \in X$, $f_{x}$ é $\nu$-integrável, e para $\nu$-quase todo $y \in Y, f_{y}$ é $\mu$-integrável. As funções definidas quase sempre $x \mapsto \int f_{x} \mathrm{~d} \nu$ e $y \mapsto \int f_{y} \mathrm{~d} \mu$ são integráveis, e $\int f \mathrm{~d}(\mu \times \nu)$ pode ser calculada pelas integrais iteradas:

$$
\int f \mathrm{~d}(\mu \times \nu)=\int\left(\int f_{x} \mathrm{~d} \nu\right) \mathrm{d} \mu(x)=\int\left(\int f_{y} \mathrm{~d} \mu\right) \mathrm{d} \nu(y) .
$$

Observação 2.40. Se $\mu$ e $\nu$ forem ambas $\sigma$-finitas, então $\mu \times \nu$ também o é, de modo que a hipótese de $\sigma$-finitude nas partes ii e iii do enunciado acima são automaticamente satisfeitas. Além disso, toda função mensurável não negativa é integrável, de modo que a parte iii vale para tais funções (o que corresponde ao teorema de Tonelli clássico).

Exemplo 2.41. A medida de Lebesgue $\mathcal{L}^{n}$ em $\mathbb{R}^{n}$ coincide com o produto das medidas exteriores $\left(\mathcal{L}^{1}\right)^{n}=\mathcal{L}^{1} \times \cdots \times \mathcal{L}^{1}$.

\subsection{Teorema de Severini-Egorov}

Devido a Carlo Severini, um matemático italiano, e Dmitri Egorov, um físico e geômetra russo, o teorema de Severini-Egorov estabelece uma condição para a convergência quase uniforme de uma sequência de funções mensuráveis num espaço de medida finita.

Definição 2.42 (convergência quase uniforme). Sejam $\mu$ uma medida num conjunto $X, Y$ um espaço métrico e $A \subset X$. Dizemos que uma sequência $\left(f_{n}\right)_{n \in \mathbb{N}}$ de funções $\mu$-mensuráveis com valores em $Y$ converge quase uniformemente em $A$ para uma função $f: \operatorname{dom} f \subset X \rightarrow Y$ se, para todo $\epsilon>0$, existe $B \in \sigma(\mu)$ tal que $\mu(A \backslash B)<\epsilon$ e $\left(f_{n}\right)_{n \in \mathbb{N}}$ converge uniformemente para $f$ em $B$.

Teorema 2.43 (Severini-Egorov, [9], [7]). Sejam $\mu$ uma medida sobre um conjunto $X, Y$ um espaço métrico separável, $A \subset X \operatorname{com} \mu(A)<\infty e\left(f_{n}\right)_{n \in \mathbb{N}}$ uma sequência de funções $\mu$-mensuráveis com valores em $Y$, a qual converge pontualmente $\mu$-q.s. em A para uma função $f: \operatorname{dom} f \subset X \rightarrow Y$ $\mu$-mensurável. Então $\left(f_{n}\right)_{n \in \mathbb{N}}$ converge quase uniformemente para $f$ em A.

O teorema acima diz respeito a convergência sequências de funções mensuráveis. No teorema 4.21 do capítulo 4, sobre retificabilidade a partir da convergência de blowups, precisaremos da seguinte versão do teorema de Severini-Egorov para famílias de funções mensuráveis; este teorema foi obtido a partir da adaptação de um argumento de F. S. Cassano.

Teorema 2.44 (Severini-Egorov para famílias de funções). Sejam $\mu$ uma medida num conjunto $X, Y$ um espaço métrico separável, $A \subset X \operatorname{com} \mu(A)<\infty,\left(f_{r}\right)_{r>0}$ uma família de funções $\mu$ mensuráveis $X \rightarrow Y$ e $f: X \rightarrow Y \mu$-mensurável tais que 
i) $f_{r} \stackrel{r \rightarrow 0}{\rightarrow} f \mu$-q.s. em $A$;

ii) para $\mu$-quase todo $x \in X, r \in(0, \infty) \mapsto f_{r}(x) \in Y$ é contínua à esquerda ou contínua à direita.

Então $\left(f_{r}\right)_{r>0}$ converge para $f$ quase uniformemente em $A$, i.e. para todo $\epsilon>0$, existe $B \in \sigma(\mu)$ tal que $\mu(A \backslash B)<\epsilon$ e $f_{r} \stackrel{r \rightarrow 0}{\rightarrow} f$ uniformemente em $B$.

Demonstração. Denote por $d$ a métrica de $Y$. Para cada $i \in \mathbb{N}$, seja $g_{i}: X \rightarrow[0, \infty]$ dada por

$$
g_{i}(x):=\sup \left\{d\left(f_{r}(x), f(x)\right) \mid 0<r<1 / i\right\} .
$$

Em vista da hipótese ii), para $\mu$-q.t. $x \in X$, para todo $i \in \mathbb{N}$ e todo $0<r<1 / i, f_{r}(x)$ é o limite de uma sequência $\left\{f_{q_{j}}(x)\right\}_{j \in \mathbb{N}} \operatorname{com} q_{j} \in \mathbb{Q} \cap(0,1 / i)$ para todo $j \in \mathbb{N}$. Daí, para $\mu$-q.t. $x \in X$,

$$
g_{i}(x):=\sup \left\{d\left(f_{r}(x), f(x)\right) \mid r \in \mathbb{Q}, 0<r<1 / i\right\} .
$$

Como o segundo membro da igualdade acima define uma função $\mu$-mensurável $X \rightarrow[0, \infty]$ (pois, para $r$ fixo, $x \in X \mapsto d\left(f_{r}(x), f(x)\right)$ é $\mu$-mensurável; é para garantir isso que usamos a separabilidade de $Y)$, conclui-se que $g_{i}: X \rightarrow[0, \infty]$ é $\mu$-mensurável para todo $i \in \mathbb{N}$. Além disso, pela hipótese i), $\left(g_{i}\right)_{i \in \mathbb{N}}$ converge pontualmente $\mu$-q.s. para 0 em $A$. Daí, pelo teorema de Severini-Egorov clássico (i.e. para sequências de funções - veja 2.43$)$, conclui-se que $\left(g_{i}\right)_{i}$ converge para zero quase uniformemente em $A$, i.e. para todo $\epsilon>0$, existe $B \in \sigma(\mu)$ tal que $\mu(A \backslash B)<\epsilon$ e $g_{i} \rightarrow 0$ uniformemente em $B$, logo $\left(f_{r}\right)_{r>0}$ converge uniformemente para $f$ em $B$ para $r \rightarrow 0$.

\subsection{Construção de Carathéodory e Medidas de Hausdorff}

Com o objetivo de se introduzir as chamadas medidas de Hausdorff, partiremos de uma construção abstrata num espaço métrico $X$, a qual pode ser usada para se gerar uma pletora de medidas de Borel em $X$ com motivação geométrica. Introduziremos aqui apenas as medidas de Hausdorff, mas o leitor interessado poderá consultar, por exemplo, [9] ou [17] para outras tais medidas.

Sejam $X$ um espaço métrico, $\mathcal{F} \subset 2^{X}$ e uma função $\zeta: \mathcal{F} \rightarrow[0, \infty]$. A grosso modo, a ideia é "medir" os elementos de $\mathcal{F}$ a partir do medidor $\zeta$, e usar isso para definir uma medida de Borel em $X$. Definimos tal medida em duas etapas:

1) Para $0<\delta \leq \infty$ definimos, $\forall A \subset X$,

$$
\psi_{\delta}(A):=\inf \left\{\sum_{S \in \mathcal{G}} \zeta(S) \mid \mathcal{G} \subset \mathcal{F} \cap\{S \mid \text { diam } S \leq \delta\}, \mathcal{G} \text { cobertura enumerável de } A\right\} .
$$

Lembre que $\inf \emptyset=\infty$, de modo que $\psi_{\delta}(A)=\infty$ se não houver uma cobertura enumerável de $A$ por elementos de $\mathcal{F}$ com diâmetro $\leq \delta$. Além disso, se $A=\emptyset$, então $\mathcal{G}=\emptyset \subset \mathcal{F}$ é uma tal cobertura para $A$ e, uma vez que a soma sobre a família vazia é zero, concluímos que $\psi_{\delta}(\emptyset)=0$. Agora note que, se $V \subset W \subset X$, então qualquer cobertura enumerável de $W$ é uma cobertura enumerável de $V, \operatorname{logo} \psi_{\delta}(V) \leq \psi_{\delta}(W)$, ou seja, $\psi_{\delta}$ é monótona. A verificação de que $\psi_{\delta}$ é enumeravelmente subaditiva também é direta; portanto, $\psi_{\delta}$ é uma medida em $X$. Em geral, as medidas $\left(\psi_{\delta}\right)_{\delta}$ assim definidas não são Borel, mas podemos fabricar uma medida de Borel a partir delas, como fazemos na segunda etapa da construção:

2) Defina, para cada $A \subset X, \psi(A):=\sup \left\{\psi_{\delta}(A) \mid 0<\delta \leq \infty\right\} \in[0, \infty]$.

Note que, para cada $A \subset X$ fixado, $\left(\psi_{\delta}(A)\right)_{\delta}$ é decrescente em $\delta$, de modo que o sup na definição acima coincide com $\lim _{\delta \rightarrow 0} \psi_{\delta}(A)$.

Definição 2.45. Com a notação acima, dizemos que $\psi$ é o resultado da Construção de Carathéodory a partir do método $\zeta$ sobre $\mathcal{F}$. Chamamos $\psi_{\delta}$ de $\delta$-aproximação de $\psi$. 
A verificação de que $\psi$ é uma medida em $X$ é direta, e o critério de Carathéodory 2.17 garante que esta medida é boreliana. Isso prova parte da seguinte proposição:

Proposição 2.46 ([9], [7]). Sejam X um espaço métrico e $\psi$ o resultado da construção de Carathéodory a partir do método $\zeta$ sobre $\mathcal{F} \subset 2^{X}$. Então $\psi$ é uma medida boreliana. Além disso, se $\mathcal{F} \subset \mathscr{B}_{X}$, a medida $\psi$ é Borel-regular.

Definição 2.47 (medidas de Hausdorff). Sejam $X$ um espaço métrico e $m \in \mathbb{R}$ não-negativo. Tome $\mathcal{F}=2^{X}$ e $\zeta: 2^{X} \rightarrow[0, \infty]$ dado por

$$
\zeta(S):=\alpha(m) \frac{(\operatorname{diam} S)^{m}}{2^{m}},
$$

onde $\alpha(m):=\frac{\pi^{\frac{m}{2}}}{\Gamma\left(\frac{m}{2}+1\right)}$.

O resultado da construção de Carathéodory para $\zeta$ sobre $2^{X}$ é chamado de medida m-dimensional de Hausdorff em $X$, denotada por $\mathcal{H}^{m}$. Usamos a notação $\mathcal{H}_{\delta}^{m}$ para a $\delta$-aproximação de $\mathcal{H}^{m}$.

Note que, para $m$ inteiro, $\alpha(m)$ é o volume euclidiano da bola unitária de $\mathbb{R}^{m}$. A medida de Hausdorff representa, nesse sentido, um "volume euclidiano $m$-dimensional".

Definição 2.48 (aplicações lipschitzianas e constante de Lipschitz). Sejam $X$ e $Y$ espaços métricos. Uma aplicação $f: X \rightarrow Y$ é chamada lipschitziana ou Lipschitz se existir $C \geq 0$ tais que, $\forall x, y \in X$, $d_{Y}(f(x), f(y)) \leq C d_{X}(x, y)$. Se $f$ é Lipschitz, existe a menor constante $C$ com a propriedade acima chama-se constante de Lipschitz de $f$, denotada por Lip $f$ e é dada por:

$$
\operatorname{Lip} f:=\sup \left\{\frac{d_{Y}(f(x), f(y))}{d_{X}(x, y)} \mid x \neq y \in X\right\}
$$

Proposição 2.49 (propriedades imediatas das medidas de Hausdorff, [9], [12] [7]). Sejam X um espaço métrico e $m \in \mathbb{R}$ não negativo. As seguintes propriedades são válidas para $\mathcal{H}^{m}$ :

i) A medida de Hausdorff é compativel com a operação de tomar traços (cf. 2.13). Ou seja, se X é um espaço métrico e $A \subset X$, o traço de $\mathcal{H}^{m}$ em $A$ coincide com a medida m-dimensional de Hausdorff em $A$ (como um subespaço métrico de $X$ ).

ii) A medida de Hausdorff é invariante por isometrias. Ou seja, se $Y$ é outro espaço métrico e $f: X \rightarrow Y$ é uma isometria sobre $Y$, então o pushforward $f_{\#} \mathcal{H}^{m}$ coincide com a medida $m$-dimensional de Hausdorff em $Y$. Mais geralmente, se $f$ for uma isometria a valores em $Y$, não necessariamente sobrejetiva, então, para todo $A \subset X, \mathcal{H}^{m}(f(A))=\mathcal{H}^{m}(A)$.

iii) Se $Y$ é outro espaço métrico e $f: X \rightarrow Y$ tem constante de Lipschitz Lip $f<\infty$, então, $\forall A \subset X, \mathcal{H}^{m}(f(A)) \leq(\operatorname{Lip} f)^{m} \mathcal{H}^{m}(A)$.

iv) $\mathcal{H}^{m}$ também coincide com o resultado da construção de Carathéodory para $\xi$ (mesmo método da definição 2.47) sobre $\mathcal{F}^{\prime}=\{$ subconjuntos fechados de $X\}$ ou $\mathcal{F}^{\prime \prime}=\{$ subconjuntos abertos de $X\}$. Se $X$ é um espaço vetorial normado, também podemos considerar $\mathcal{F}^{\prime \prime \prime}=\{$ subconjuntos convexos fechados de $X\}$.

v) $\mathcal{H}^{m}$ é uma medida de Borel regular em $X$.

vi) $\mathcal{H}^{0}$ coincide com a medida de contagem em $X$.

A seguinte proposição é uma preparação para a introdução da noção de dimensão de Hausdorff.

Proposição 2.50 ([9], [12] [7]). Sejam X um espaço métrico, $A \subset X$ e $0 \leq s<t<\infty$. Se $\mathcal{H}^{s}(A)<\infty$ então $\mathcal{H}^{t}(A)=0$. 
Definição 2.51 (dimensão de Hausdorff). Sejam $X$ um espaço métrico e $A \subset X$. O número real estendido $\inf \left\{m \in[0, \infty] \mid \mathcal{H}^{m}(A)=0\right\}=\sup \left\{m \in[0, \infty] \mid \mathcal{H}^{m}(A)=\infty\right\} \in[0, \infty]$ é chamado dimensão de Hausdorff de $A$, denotado por $\mathcal{H}$-dim $A$.

Proposição 2.52 (propriedades imediatas da dimensão de Hausdorff, [9], [7]). Seja X um espaço métrico.

a) Se $Y \subset X$ é um subespaço métrico de $X$ e $A \subset Y$, a dimensão de Hausdorff de $A$ como subconjunto do espaço métrico $Y$ coincide com a dimensão de Hausdorff de A como subconjunto do espaço métrico $X$.

b) A dimensão de Hausdorff é invariante por isometrias, i.e. se $Y$ é um espaço métrico, $f: X \rightarrow Y$ uma isometria (não necessariamente sobrejetiva) e $A \subset X$, então $\mathcal{H}$-dim $A=\mathcal{H}$-dim $f(A)$.

c) Sejam $X, Y$ espaços métricos e $f: X \rightarrow Y$ uma aplicação Lipschitz. Para todo $A \subset X$, $\mathcal{H}$-dim $f(A) \leq \mathcal{H}$-dim $A$. Em particular, se $f$ for bi-Lipschitz sobre sua imagem (i.e. $f$ é Lipschitz e tem uma inversa Lipschitz $\left.f^{-1}: \operatorname{Im} f \rightarrow X\right)$, então $\forall A \subset X, \mathcal{H}$-dim $f(A)=\mathcal{H}$-dim $A$.

d) (monotonicidade) Se $A \subset B \subset X$, então $\mathcal{H}$-dim $A \leq \mathcal{H}$-dim $B$.

e) (estabilidade com respeito a uniões enumeráveis) $S e A=\cup_{n \in \mathbb{N}} A_{n} \subset X$, então $\mathcal{H}$-dim $A=$ $\sup \left\{\mathcal{H}\right.$-dim $\left.A_{n} \mid n \in \mathbb{N}\right\}$.

Em $\mathbb{R}^{n}$, a medida de Hausdorff $n$-dimensional coincide com a medida de Lebesgue $\mathcal{L}^{n}$. Isso é uma consequência da desigualdade isodiamétrica, enunciada abaixo, que também tem importância per se.

Teorema 2.53 (desigualdade isodiamétrica, [12], [7]). A medida de Lebesgue de todo subconjunto de $\mathbb{R}^{n}$ é menor ou igual à de uma bola euclidiana de mesmo diâmetro. Ou seja, para todo $A \subset \mathbb{R}^{n}$,

$$
\mathcal{L}^{n}(A) \leq \alpha(n)\left(\frac{\operatorname{diam} A}{2}\right)^{n} .
$$

Teorema 2.54. Para todo $\delta \in(0, \infty]$ e $n \in \mathbb{N}, \mathcal{H}^{n}=\mathcal{H}_{\delta}^{n}=\mathcal{L}^{n}$ em $\mathbb{R}^{n}$.

Corolário 2.55. $\mathcal{H}$-dim $\mathbb{R}^{n}=n$.

Demonstração. Aplique a estabilidade da dimensão de Hausdorff com respeito a uniões enumeráveis, cf. proposição 2.52.e), para $\mathbb{R}^{n}=\cup_{k \in \mathbb{N}} C_{k}$, onde cada $C_{k}$ é um cubo não degenerado com medida de Lebesgue finita, i.e. $0<\mathcal{H}^{n}\left(C_{k}\right)<\infty$, de modo que $\forall k \in \mathbb{N}$, $\mathcal{H}$-dim $C_{k}=n$.

Corolário 2.56. Se E é um subespaço vetorial k-dimensional de um espaço normado $X$, então $\mathcal{H}$-dim $E=k$.

Demonstração. Seja $f: \mathbb{R}^{k} \rightarrow X$ um isomorfismo linear com $\operatorname{Im} f=E$. Então $f$ é bi-Lipschitz sobre sua imagem. Portanto, segue da proposição 2.52.c) que $\mathcal{H}$-dim $E=\mathcal{H}$-dim $\mathbb{R}^{k}=k$.

Mais geralmente, será provado como aplicação da fórmula da área em espaços euclidianos que, para toda subvariedade riemanniana $k$-dimensional $\mathrm{M} \subset \mathbb{R}^{n}$, a medida induzida pela métrica riemanniana em $\mathrm{M}$ coincide com o traço $\left.\mathcal{H}^{k}\right|_{\mathrm{M}}$, o que implica $\mathcal{H}$-dim $M=k$.

\subsection{Teoremas de Cobertura}

\subsubsection{Teorema de Cobertura de Vitali}

Notação. Dados uma bola fechada $B=\mathbb{B}(x, r)$ em $\mathbb{R}^{n}$ e $0<t<\infty$, definimos

$$
t B:=\mathbb{B}(x, t r) .
$$


Num espaço métrico arbitrário $X$, no entanto, os centros e os raios das bolas não ficam univocamente determinados (tome, por exemplo, $[0, \infty)$ como subespaço métrico de $\mathbb{R}$ e olhe as bolas centradas no 0). Nesse caso, dada uma bola fechada $B \subset X$, para definir $t B$, poderíamos escolher um centro $x$ e um raio $r$ e proceder como acima, i.e.poderíamos considerar $x$ e $r$ como sendo dados ao ser dada a bola; alternativamente, preferimos proceder como em [17] e definir, digamos, para $t=5$ :

$$
5 B:=\cup\left\{B^{\prime} \subset X \text { Bola fechada } \mid B^{\prime} \cap B \neq \emptyset \text {, diam } B^{\prime} \leq 2 \operatorname{diam} B\right\},
$$

o que claramente coincide com a definição anterior caso $X=\mathbb{R}^{n}$.

Teorema 2.57 (teorema de cobertura de Vitali, [9], [13], [12], [7]). Sejam X um espaço métrico e $\mathcal{F} \subset 2^{X}$ uma familia de bolas fechadas e não degeneradas em $X$, tal que $\sup \{\operatorname{diam} B \mid B \in \mathcal{F}\}<\infty$. Então existe uma subfamília disjunta $\mathcal{G} \subset \mathcal{F}$ tal que $\cup_{B \in \mathcal{F}} B \subset \cup_{B \in \mathcal{G}} 5 B$.

Observação 2.58. Com a notação do teorema 2.57, note que, se $X$ for separável, então $\mathcal{G}$ é enumerável (uma vez que qualquer família disjunta de subconjuntos de $X$ com interiores não vazios é enumerável).

Definição 2.59 (cobertura fina). Sejam $X$ um espaço métrico, $\mathcal{F} \subset 2^{X}$ e $A \subset X$. Diz-se que $\mathcal{F}$ é uma cobertura fina de $A$, ou que $\mathcal{F}$ cobre $A$ finamente, se $\mathcal{F}$ for uma cobertura de $A$ tal que, $\forall x \in A, \inf \{\operatorname{diam} B \mid x \in B \in \mathcal{F}\}=0$.

Corolário 2.60 ([9], [13], [12], [7]). Sejam X um espaço métrico, $A \subset X, \mathcal{F} \subset 2^{X}$ uma família de bolas fechadas não degeneradas de $X$ que cubra $A$ finamente. Então existe uma subfamília disjunta $\mathcal{G} \subset \mathcal{F}$ tal que, para todo $F \subset \mathcal{F}$ finito, $A \backslash \cup_{B \in F} B \subset \cup_{B \in \mathcal{G} \backslash F} 5 B$.

Corolário 2.61 ([9], [13], [12], [7]). Sejam $A \subset \mathbb{R}^{n}$ e $\mathcal{F}$ uma coleção de bolas fechadas não degeneradas em $\mathbb{R}^{n}$ que cubra $A$ finamente. Então, para todo $\epsilon>0$, existe uma subfamília disjunta $\mathcal{G} \subset \mathcal{F}$ tal que $\mathcal{L}^{n}(\cup \mathcal{G}) \leq \mathcal{L}^{n}(A)+\epsilon$ and $\mathcal{L}^{n}(A \backslash \cup \mathcal{G})=0$.

Corolário 2.62 (preenchendo abertos com bolas fechadas com respeito à medida de Lebesgue, [9], [13], [12], [7]). Sejam $U \subset \mathbb{R}^{n}$ um aberto e $\mathcal{F}$ uma família de bolas fechadas não degeneradas contidas em $U$ que cubra $U$ finamente (por exemplo, é o caso se $\mathcal{F}$ é a família de todas as bolas fechadas não degeneradas contidas em $U$, ou a família de todas as tais bolas com diâmetros limitados por uma constante $\delta>0$ ). Então existe uma subfamília disjunta $\mathcal{G} \subset \mathcal{F}$ tal que $\mathcal{L}^{n}(U \backslash \cup \mathcal{G})=0$.

\subsubsection{Teorema de Cobertura de Besicovitch}

Teorema 2.63 (Teorema de cobertura de Besicovitch, [12]). Para cada $n \in \mathbb{N}$, existe uma constante natural $N=N(n)$, dependendo apenas da dimensão $n$ do espaço euclidiano $\mathbb{R}^{n}$, tal que a seguinte propriedade é satisfeita: se $\mathcal{F}$ for qualquer família de bolas fechadas não degeneradas em $\mathbb{R}^{n}$ com $\sup \{\operatorname{diam} B \mid B \in \mathcal{F}\}<\infty$, e se $A$ é o conjunto dos centros das bolas em $\mathcal{F}$, então existem $\mathcal{G}_{1}, \cdots, \mathcal{G}_{N} \subset \mathcal{F}$ tais que , para $1 \leq i \leq N, \mathcal{G}_{i}$ é uma subfamília disjunta de $\mathcal{F}$ e $\cup_{i=1}^{N} \mathcal{G}_{i}$ cobre $A$.

Definição 2.64. Sejam $X$ um espaço métrico, $\mathcal{F}$ uma coleção de bolas em $X$ e $A \subset X$. Diz-se que $\mathcal{F}$ é uma cobertura fina no sentido forte de $A$, ou que $\mathcal{F}$ cobre $A$ finamente no sentido forte, se $\mathcal{F}$ for uma cobertura de $A$ tal que, $\forall x \in A, \inf \{r>0 \mid \mathbb{B}(x, r) \in \mathcal{F}\}=0$.

Claramente, toda cobertura fina de $A$ no sentido forte é uma cobertura fina de $A$ no sentido da definição 2.59 , mas a recíproca não vale.

Corolário 2.65 (preenchendo abertos com bolas fechadas com respeito a uma medida boreliana, [12]). Sejam $\mu$ uma medida de Borel em $\mathbb{R}^{n}, A \subset \mathbb{R}^{n}$ com $\mu(A)<\infty$ e $\mathcal{F}$ uma família de bolas fechadas não degeneradas que cubra A finamente no sentido forte. Então, para qualquer conjunto aberto $U \supset A$, existe uma subfamília disjunta enumerável $\mathcal{G} \subset \mathcal{F}$ tal que $\cup \mathcal{G} \subset U$ e

$$
\mu(A \backslash \cup \mathcal{G})=0
$$




\subsection{Teoremas de Densidade e Diferenciação de Medidas}

\subsubsection{Densidades}

Definição 2.66 (densidades $n$-dimensionais). Sejam $A \subset X, x \in X, n>0$ real e $\mu$ uma medida em $X$. Definimos:

1) a densidade superior $n$-dimensional de $A$ em $x$ com respeito a $\mu$ :

$$
\Theta^{* n}(\mu, A, x):=\limsup _{r \rightarrow 0} \frac{\mu(A \cap \mathbb{B}(x, r))}{\alpha(n) r^{n}} \in[0, \infty] .
$$

2) a densidade inferior $n$-dimensional de $A$ em $x$ com respeito a $\mu$ :

$$
\Theta_{*}^{n}(\mu, A, x):=\liminf _{r \rightarrow 0} \frac{\mu(A \cap \mathbb{B}(x, r))}{\alpha(n) r^{n}} \in[0, \infty] .
$$

Se $\Theta^{* n}(\mu, A, x)=\Theta_{*}^{n}(\mu, A, x)$, denotamos o valor comum por $\Theta^{n}(\mu, A, x)$, o qual é chamado densidade de $A$ em $x$ com respeito a $\mu$.

Para $A=X$, usamos as notações $\Theta^{* n}(\mu, x), \Theta_{*}^{n}(\mu, x)$ e $\Theta^{n}(\mu, x)$ para $\Theta^{* n}(\mu, X, x), \Theta_{*}^{n}(\mu, X, x)$ e $\Theta^{n}(\mu, X, x)$, respectivamente.

Note que não se assume $A$ mensurável.

Observação 2.67. Com a notação acima:

1) Note que $\Theta^{* n}(\mu, A, x)=\Theta^{* n}\left(\mu\llcorner A, x)\right.$ e $\Theta_{*}^{n}(\mu, A, x)=\Theta_{*}^{n}(\mu\llcorner A, x)$.

2) Se $U \subset X$ é aberto e $x \in U, \Theta^{* n}(\mu, A, x)=\Theta^{* n}\left(\mu\llcorner U, A, x)\right.$ e $\Theta_{*}^{n}(\mu, A, x)=\Theta_{*}^{n}(\mu\llcorner U, A, x)$.

Teorema 2.68 (teorema de comparação de densidade). Sejam $\mu$ uma medida num espaço métrico $X, n>0$ real e $t \geq 0$.

i) $[9,2.10 .19(1)]$ Se $\mu$ for regular, $A \subset X e, \forall x \in A, \Theta^{* n}(\mu, x) \leq t$ então $\mu(A) \leq 2^{n} t \mathcal{H}^{n}(A)$.

ii) [13], [7] Se $\mu$ for boreliana, $A \subset A_{1} \subset X e, \forall x \in A, \Theta^{* n}\left(\mu, A_{1}, x\right) \geq t$ então $t \mathcal{H}^{n}(A) \leq \mu\left(A_{1}\right)$.

Teorema 2.69 (teorema da densidade superior, [13], [7]). Sejam $\mu$ uma medida Borel-regular num espaço métrico $X, n>0$ real e $B \in \sigma(\mu)$. Se $\mu(B)<\infty$, ou se $\mu$ for aberta $\sigma$-finita, então $\Theta^{* n}(\mu, B, x)=0$ para $\mathcal{H}^{n}$-q.t. $x \in X \backslash B$.

Corolário 2.70 (teorema de densidade para a medida de Lebesgue, [13], [7]). Se $B \subset \mathbb{R}^{n}$ é $\mathcal{L}^{n}$ mensurável, então $\Theta^{n}\left(\mathcal{L}^{n}, B, x\right)$ existe para $\mathcal{L}^{n}$-a.e. $x \in \mathbb{R}^{n}, \Theta^{n}\left(\mathcal{L}^{n}, B, x\right)=1$ para $\mathcal{L}^{n}$-a.e. $x \in B$ e $\Theta^{n}\left(\mathcal{L}^{n}, B, x\right)=0$ para $\mathcal{L}^{n}$-a.e. $x \in \mathbb{R}^{n} \backslash B$.

\subsubsection{Densidades Relativas e Teoremas de Diferenciação}

Definição 2.71 (densidades relativas). Sejam $X$ um espaço métrico, $\mu$ e $\nu$ medidas em $X$, e $x \in X$. Definimos a densidade superior e inferior de $\mu$ com respeito a $\nu$ em $x$, respectivamente, por:

$$
\begin{gathered}
\Theta^{* \nu}(\mu, x):=\limsup _{r \rightarrow 0} \frac{\mu(\mathbb{B}(x, r))}{\nu(\mathbb{B}(x, r))} \in[0, \infty], \\
\Theta_{*}^{\nu}(\mu, x):=\liminf _{r \rightarrow 0} \frac{\mu(\mathbb{B}(x, r))}{\nu(\mathbb{B}(x, r))} \in[0, \infty],
\end{gathered}
$$

onde adotamos as regras aritméticas estendidas $\frac{0}{0}:=0, \frac{\infty}{\infty}:=0$. Se $\Theta^{* \nu}(\mu, x)=\Theta_{*}^{\nu}(\mu, x)$, dizemos que a densidade de $\mu$ com respeito a $\nu$ em $x$ existe e a denotamos por $\Theta^{\nu}(\mu, x):=\Theta^{* \nu}(\mu, x)=$ $\Theta_{*}^{\nu}(\mu, x)$. 
Definição 2.72 (Propriedade de Vitali Simétrica(SVP)). Dizemos que uma medida $\mu$ num espaço métrico $X$ satisfaz a propriedade de Vitali simétrica se, para todo $A \subset X \operatorname{com} \mu(A)<\infty$ e para toda cobertura fina no sentido forte $\mathcal{F}$ de $A$ (cf. 2.64) por bolas fechadas não degeneradas, existe uma subfamília disjunta enumerável $\mathcal{G} \subset \mathcal{F}$ tal que $\mu(A \backslash \cup \mathcal{G})=0$.

Proposição 2.73 ([7]). Seja $X$ um subespaço métrico de $\mathbb{R}^{n}$ e $\mu$ uma medida boreliana em $X$. Então $\mu$ satisfaz a propriedade de Vitali simétrica 2.72.

Demonstração. Se $X=\mathbb{R}^{n}$, a tese decorre diretamente do corolário 2.65 do teorema de cobertura de Besicovitch. No caso geral, seja $\rho$ a métrica em $X$ induzida pela métrica euclidiana $d$ em $\mathbb{R}^{n}$ e $i: X \rightarrow \mathbb{R}^{n}$ a inclusão. Usaremos sobrescritos $\rho$ e $d$ para bolas em $X$ e $\mathbb{R}^{n}$, respectivamente, de modo que, para todo $x \in X, \mathbb{B}(x, r)^{\rho}=\mathbb{B}(x, r)^{d} \cap X$. Dados $A \subset X \operatorname{com} \mu(A)<\infty$ e $\mathcal{F} \subset$ $\mathscr{P}(X)$ uma cobertura fina no sentido forte de $A$ por bolas fechadas não degeneradas, seja $\mathcal{F}^{\prime}:=$ $\{B$ bola fechada não degenerada $\mid B \cap X \in \mathcal{F}\}$. Então $\mathcal{F}^{\prime} \subset \mathscr{P}\left(\mathbb{R}^{n}\right)$ é uma cobertura fina no sentido forte de $A$ em $\mathbb{R}^{n}$; de fato, para todo $x \in A$, e para todo $\delta>0$, o fato de que $\mathcal{F}$ é uma cobertura fina no sentido forte de $A$ em $X$ garante a existência de $0<r<\delta$ tal que $\mathbb{B}(x, r)^{d} \cap X=\mathbb{B}(x, r)^{\rho} \in \mathcal{F}$, portanto $\mathbb{B}(x, r)^{d} \in \mathcal{F}^{\prime}$ por definição. Como a medida $i_{\#} \mu$ é uma medida boreliana em $\mathbb{R}^{n}$, pela proposição 2.27 , segue do caso já provado que existe um subfamília disjunta enumerável $\mathcal{G}^{\prime}=\mathcal{F}^{\prime}$ tal que $i_{\#} \mu\left(A \backslash \cup \mathcal{G}^{\prime}\right)=0$. Definimos $\mathcal{G}:=\left\{B \cap X \mid B \in \mathcal{G}^{\prime}\right\}$; então $\mathcal{G}$ é uma subfamília disjunta enumerável de $\mathcal{F}$ e, como para todo $B \in \mathcal{G}^{\prime}, A \backslash B=A \backslash(B \cap X)$, segue que $A \backslash \cup \mathcal{G}=A \backslash \cup \mathcal{G}^{\prime}$. Assim, $\mu(A \backslash \cup \mathcal{G})=i_{\#} \mu\left(A \backslash \cup \mathcal{G}^{\prime}\right)=0$, o que conclui a demostração.

Teorema 2.74 (teorema de diferenciação de Lebesgue, [13], [7]). Sejam $\mu$ uma medida Borel-regular aberta $\sigma$-finita num espaço métrico $X$ satisfazendo $S V P$ e $f: X \rightarrow \mathbb{C}$ uma função $\mu$-mensurável satisfazendo uma das condições abaixo:

i) $f \in \mathrm{L}^{1}(\mu)$ ou

ii) $X$ separável e $f \in \mathrm{L}_{\text {loc }}^{1}(\mu)$, i.e. $\forall x \in X, \exists r>0, \int_{\mathbb{B}(x, r)}|f| \mathrm{d} \mu<\infty$.

Então, para $\mu$-q.t. $x \in X$ :

$$
\lim _{r \rightarrow 0} \frac{1}{\mu(\mathbb{B}(x, r))} \int_{\mathbb{B}(x, r)} f \mathrm{~d} \mu=f(x) .
$$

Corolário 2.75 (pontos de Lebesgue, [13], [7]). Sejam X um espaço métrico separável, $\mu$ uma medida Borel-regular aberta $\sigma$-finita em $X$ satisfazendo $S V P, 1 \leq p<\infty$ e $f \in \mathrm{L}_{\mathrm{loc}}^{\mathrm{p}}(\mu)$, i.e. $\forall x \in X, \exists r>0, \int_{\mathbb{B}(x, r)}|f|^{p} \mathrm{~d} \mu<\infty$. Então, para $\mu$-q.t. $x \in X$,

$$
\lim _{r \rightarrow 0} \frac{1}{\mu(\mathbb{B}(x, r))} \int_{\mathbb{B}(x, r)}|f(y)-f(x)|^{p} \mathrm{~d} \mu(y)=0 .
$$

Definição 2.76 (pontos de Lebesgue). Com a notação do corolário anterior, um ponto $x \in X$ para o qual vale (2.5) é chamado $p$-ponto de Lebesgue de $f$ com respeito a $\mu$; para $p=1$ dizemos, simplesmente, ponto de Lebesgue de $f$.

Claramente, todo ponto de continuidade de $f$ é um ponto de Lebesgue de $f$.

Definição 2.77 (continuidade absoluta e singularidade mútua). Sejam $\mu$ e $\nu$ medidas borelianas num espaço topológico $X$. Dizemos que:

1) $\mu$ é absolutamente contínua com respeito a $\nu$ (notação: $\mu \ll \nu$ ) se, para todo $A \subset X, \nu(A)=0$ implica $\mu(A)=0$;

2) $\mu$ e $\nu$ são mutuamente singulares (notação: $\mu \perp \nu$ ) se existe $A \in \mathscr{B}_{X}$ tal que $\mu$ é concentrada em $A$ e $\nu$ é concentrada em $X \backslash A$. 
Teorema 2.78 (teorema de decomposição de Lebesgue, [13], [7]). Sejam $\mu$ uma medida boreliana $\sigma$-finita e $\nu$ uma medida Borel-regular num espaço métrico $X$. Então existe $B \in \mathscr{B}_{X}$ tal que $\nu$ é concentrada em $B^{c}$ e $\mu\left\llcorner B^{c} \ll \nu\right.$, de modo que

$$
\mu=\mu\left\llcorner B+\mu\left\llcorner B^{c}, \quad \mu\left\llcorner B \perp \nu, \mu\left\llcorner B^{c} \ll \nu .\right.\right.\right.\right.
$$

Além disso:

1) $B \in \mathscr{B}_{X}$ satisfazendo (LD) é único a menos de conjuntos $\mu$-nulos, i.e. se $B^{\prime} \in \mathscr{B}_{X}$ também satisfizer (LD), então $B \Delta B^{\prime}$ é $\mu$-nulo.

2) a decomposição (LD) é única no sentido de que, se $\mu=\mu_{s}+\mu_{a}$ com $\mu_{s} \perp \nu$ e $\mu_{a} \ll \nu$, então $\mu_{s}=\mu\left\llcorner B\right.$ e $\mu_{a}=\mu\left\llcorner B^{c}\right.$.

Definição 2.79. Com a notação acima, chamamos $\mu\left\llcorner B\right.$ de parte singular e $\mu\left\llcorner B^{c}\right.$ de parte absolutamente continuade $\mu$ com respeito a $\nu$.

Teorema 2.80 (teorema de diferenciação para medidas borelianas em espaços métricos, [13], [7]). Sejam $\mu$ e $\nu$ medidas borelianas regulares abertas $\sigma$-finitas num espaço métrico $X$. Suponha que $X$ seja separável ou que $\nu$ seja finita nas bolas fechadas de $X$.

i) O conjunto $Y:=\left\{x \in X \mid \Theta^{* \nu}(\mu, x)=\Theta_{*}^{\nu}(\mu, x)\right\}$ é boreliano e $\Theta^{\nu}(\mu, \cdot): Y \rightarrow[0, \infty]$ é boreliana.

ii) Se $\nu$ satisfaz $S V P, Y_{f}:=\left\{x \in Y \mid \Theta^{\nu}(\mu, x)<\infty\right\}$ é um boreliano de $X$ cujo complementar é $\nu$-nulo.

iii) Se ambas $\mu$ e $\nu$ satisfazem $S V P, \mu\left(Y^{c}\right)=\nu\left(Y^{c}\right)=0$.

Teorema 2.81 (teorema de diferenciação de Lebesgue-Besicovitch-Radon-Nikodym, [13], [7]). Se$j a m \mu$ e $\nu$ medidas borelianas regulares abertas $\sigma$-finitas num espaço métrico $X$. Suponha que $X$ seja separável ou que $\nu$ seja finita nas bolas fechadas de $X$, e que $\nu$ satisfaça SVP.

i) Seja $\mu=\mu_{s}+\mu_{a}$ a decomposição de Lebesgue de $\mu$ com respeito a $\nu$, i.e. $\mu_{s}=\mu\llcorner B e$ $\mu_{a}=\mu\left\llcorner B^{c}\right.$, onde $B \in \mathscr{B}_{X}$ é dado pelo teorema 2.78. Então, para todo $A \in \mathscr{B}_{X}$,

$$
\mu_{a}(A)=\int_{A} \Theta^{\nu}(\mu, x) \mathrm{d} \nu(x),
$$

de modo que, para todo $A \in \mathscr{B}_{X}, \mu(A)=\int_{A} \Theta^{\nu}(\mu, x) \mathrm{d} \nu(x)+\mu_{s}(A)$.

ii) Se $\mu$ também satisfizer $S V P$, no teorema 2.78 podemos tomar $B^{\prime}=\left\{x \in X \mid \Theta^{\nu}(\mu, x)=\infty\right\}$ no lugar de $B$.

\subsection{Medidas de Radon a Valores Vetoriais}

Notação. Seja $X$ um espaço Hausdorff localmente compacto. Denotamos por

- $\mathrm{C}_{\mathrm{c}}\left(X, \mathbb{R}^{n}\right)$ o espaço das funções contínuas $f: X \rightarrow \mathbb{R}^{n}$ com spt $f$ compacto;

- $\mathrm{C}_{0}\left(X, \mathbb{R}^{n}\right)$ o espaço das funções contínuas $f: X \rightarrow \mathbb{R}^{n}$ que se anulam no infinito, i.e. tais que $\forall \epsilon>0, \exists K \subset X$ compacto tal que $\|f\|<\epsilon$ on $X \backslash K$.

- $\mathrm{C}_{\mathrm{b}}\left(X, \mathbb{R}^{n}\right)$ o espaço das funções contínuas limitadas $f: X \rightarrow \mathbb{R}^{n}$.

Definição 2.82 (medidas de Radon a valores em $\mathbb{R}^{n}$ ). Dizemos que um funcional linear $\mu$ : $\mathrm{C}_{\mathrm{c}}\left(X, \mathbb{R}^{n}\right) \rightarrow \mathbb{R}$ é uma medida de Radon em $X$ a valores em $\mathbb{R}^{n}$ se, para cada compacto $K \subset X$, 
a restrição de $\mu$ a $C_{c}^{K}\left(X, \mathbb{R}^{n}\right):=\left\{f \in \mathrm{C}_{\mathrm{c}}\left(X, \mathbb{R}^{n}\right) \mid\right.$ spt $\left.f \subset K\right\}$, munido da norma da convergência uniforme $\|\cdot\|_{u}$, for linear contínuo; ou seja, se $\exists C_{K} \geq 0$ tal que

$$
\sup \left\{\mu \cdot f \mid f \in \mathrm{C}_{\mathrm{c}}^{\mathrm{K}}\left(X, \mathbb{R}^{n}\right),\|f\|_{u} \leq 1\right\} \leq C_{K} .
$$

(LF cont)

Se a condição acima for satisfeita com uma constante $C \geq 0$ independente de $K$, i.e. se $\mu$ for linear contínua em $\mathrm{C}_{\mathrm{c}}\left(X, \mathbb{R}^{n}\right)$ munido da norma $\|\cdot\|_{u}$, chamamos $\mu$ uma uma medida de Radon em $X$ a valores em $\mathbb{R}^{n}$ finita.

Usaremos as notações $C_{c}\left(X, \mathbb{R}^{n}\right)^{*}$ para denotar o espaço das medidas de Radon em $X$ a valores em $\mathbb{R}^{n}$ e $C_{0}\left(X, \mathbb{R}^{n}\right)^{*}$ para denotar o espaço das medidas de Radon em $X$ a valores em $\mathbb{R}^{n}$ finitas.

Notação. Sejam $X$ espaço Hausdorff localmente compacto, $U \subset X$ aberto e $f$ uma função em $X$. Usaremos a notação $f \prec U$ com o significado de que $0 \leq f \leq 1, f \in \mathrm{C}_{\mathrm{c}}(X, \mathbb{R})$ e spt $f \subset U$.

Teorema 2.83 (de representação de Riesz para funcionais lineares positivos, [6]). Sejam X espaço Hausdorff localmente compacto e $L: \mathrm{C}_{\mathrm{c}}(X, \mathbb{R}) \rightarrow \mathbb{R}$ um funcional linear positivo, i.e. $L$ é linear $e$ $L \cdot f \geq 0$ sempre que $f \geq 0$. Então existe uma única medida de Radon $\eta$ em $X$ que representa $L$, i.e. $\forall f \in \mathrm{C}_{\mathrm{c}}(X, \mathbb{R}), L \cdot f=\int f \mathrm{~d} \eta$. Além disso, $\eta$ é dada nos abertos $U \subset X$ por

$$
\eta(U)=\sup \{L \cdot f \mid f \prec U\} .
$$

Teorema 2.84 (teorema de representação de Riesz para medidas de Radon, [7]). Sejam X um espaço Hausdorff localmente compacto e $\sigma$-compacto e $\mu: \mathrm{C}_{\mathrm{c}}\left(X, \mathbb{R}^{n}\right) \rightarrow \mathbb{R}$ uma medida de Radon em $X$ a valores em $\mathbb{R}^{n}$. Então existe uma única medida de Radon $\lambda$ em $X$ e uma aplicação boreliana $\nu: X \rightarrow \mathbb{R}^{n}$ única a menos de conjuntos $\lambda$-nulos tal que $\|\nu\|=1 \lambda$-q.s. em $X$ e, $\forall f \in \mathrm{C}_{\mathrm{c}}\left(X, \mathbb{R}^{n}\right)$,

$$
\mu \cdot f=\int\langle f, \nu\rangle \mathrm{d} \lambda
$$

onde $\langle\cdot, \cdot\rangle$ denota o produto interno usual em $\mathbb{R}^{n}$. Além disso,

i) $\forall U \subset X$ aberto,

$$
\lambda(U)=\sup \left\{\mu \cdot f \mid f \in \mathrm{C}_{\mathrm{c}}\left(X, \mathbb{R}^{n}\right),\|f\| \prec U\right\} .
$$

ii) $\mu$ é finita se, e somente se, $\lambda$ é uma medida de Radon finita; em caso afirmativo, $\|\mu\|_{\mathrm{C}_{0}\left(X, \mathbb{R}^{n}\right)^{*}}=$ $\lambda(X)$.

Definição 2.85 (variação total e decomposição polar). Sejam $X$ um espaço Hausdorff localmente compacto e $\sigma$-compacto e $\mu$ uma medida de Radon em $X$ a valores em $\mathbb{R}^{n}$. Com a mesma notação do teorema precedente, $\lambda$ é chamada a variação total de $\mu$, e o par $(\nu, \lambda)$ é chamado decomposição polar de $\mu$. Usaremos a notação $|\mu|:=\lambda$ para a variação total de $\mu$.

Definição 2.86 (integração com respeito a medidas de Radon a valores em $\mathbb{R}^{n}$ ). Sejam $X$ um espaço Hausdorff localmente compacto e $\sigma$-compacto e $\mu$ uma medida de Radon em $X$ a valores em $\mathbb{R}^{n}$, com decomposição polar $(\nu,|\mu|)$.

i) Uma aplicação boreliana $f: X \rightarrow \mathbb{R}^{n}$ diz-se somável com respeito a $\mu$ se for somável com respeito a $|\mu|$, i.e. se $f \in \mathrm{L}^{1}\left(|\mu|, \mathbb{R}^{n}\right) \equiv \mathrm{L}^{1}(|\mu|)^{n}$. Para uma tal $f$, definimos

$$
\int f \cdot \mathrm{d} \mu:=\int\langle f, \nu\rangle \mathrm{d}|\mu| \in \mathbb{R} .
$$

ii) Uma função boreliana $f: X \rightarrow \mathbb{R}$ diz-se somável com respeito a $\mu$ se for somável com respeito a $|\mu|$, i.e. se $f \in \mathrm{L}^{1}(|\mu|)$. Para uma tal $f$, definimos

$$
\int f \mathrm{~d} \mu:=\int f \nu \mathrm{d}|\mu|=\left(\int f \nu_{1} \mathrm{~d}|\mu|, \ldots, \int f \nu_{n} \mathrm{~d}|\mu|\right) \in \mathbb{R}^{n} .
$$




\subsubsection{Convergência Fraca-Estrela}

Definição 2.87. Seja $X$ um espaço métrico separável localmente compacto. Diz-se que

i) uma sequência $\left(\mu_{k}\right)_{k \in \mathbb{N}}$ em $\mathrm{C}_{\mathrm{c}}\left(X, \mathbb{R}^{n}\right)^{*}$ é fraca-estrela convergente para $\mu \in \mathrm{C}_{\mathrm{c}}\left(X, \mathbb{R}^{n}\right)^{*}$ (notação: $\left.\mu_{k} \stackrel{*}{\rightarrow} \mu\right)$ se, para toda $f \in \mathrm{C}_{\mathrm{c}}\left(X, \mathbb{R}^{n}\right), \int f \cdot \mathrm{d} \mu_{k} \rightarrow \int f \cdot \mathrm{d} \mu$;

ii) uma sequência $\left(\mu_{k}\right)_{k \in \mathbb{N}}$ em $\mathrm{C}_{0}\left(X, \mathbb{R}^{n}\right)^{*}$ é fraca-estrela convergente no sentido de medidas finitas para $\mu \in \mathrm{C}_{0}\left(X, \mathbb{R}^{n}\right)^{*}$ (notação: $\mu_{k} \stackrel{* \mathrm{f}}{\rightarrow} \mu$ ) se, para toda $f \in \mathrm{C}_{0}\left(X, \mathbb{R}^{n}\right), \int f \cdot \mathrm{d} \mu_{k} \rightarrow \int f \cdot \mathrm{d} \mu$.

Proposição 2.88 (relação entre convergência fraca-estrela e convergência fraca-estrela no sentido de medidas finitas, [7]). Sejam $X$ um espaço métrico separável localmente compacto, $\left(\mu_{k}\right)_{k \in \mathbb{N}}$ uma sequência em $\mathrm{C}_{\mathrm{c}}\left(X, \mathbb{R}^{n}\right)^{*}$ e $\mu \in \mathrm{C}_{\mathrm{c}}\left(X, \mathbb{R}^{n}\right)^{*}$. As seguintes condições são equivalentes:

i) $\mu_{k} \stackrel{*}{\rightarrow} \mu e \sup _{k \in \mathbb{N}}\left|\mu_{k}\right|(X)<\infty$.

ii) $\left(\mu_{k}\right)_{k \in \mathbb{N}}$ é uma sequência em $\mathrm{C}_{0}\left(X, \mathbb{R}^{n}\right)^{*}, \mu \in \mathrm{C}_{0}\left(X, \mathbb{R}^{n}\right)^{*}$ e $\mu_{k} \stackrel{* \mathrm{f}}{ } \mu$.

Teorema 2.89 (caracterização da convergência fraca-estrela para medidas de Radon positivas, [5], [7]). Sejam $X$ um espaço métrico separável localmente compacto, $\left(\mu_{k}\right)_{k \in \mathbb{N}}$ uma sequência de medidas de Radon positivas em $X$ e $\mu$ uma medida de Radon positiva em $X$. As seguintes condições são equivalentes:

i) $\mu_{k} \stackrel{*}{\rightarrow} \mu$.

ii) Para todo $K \subset X$ compacto e todo $U \subset X$ aberto,

$$
\mu(K) \geq \limsup \mu_{k}(K) \quad \text { and } \quad \mu(U) \leq \liminf \mu_{k}(U) .
$$

iii) Para todo $E \in \mathscr{B}_{X}$ relativamente compacto tal que $\mu(\partial E)=0, \mu_{k}(E) \rightarrow \mu(E)$.

Além disso, se $\mu_{k} \stackrel{*}{\rightarrow} \mu$ e $x \in \operatorname{spt} \mu$, existe $n \in \mathbb{N}$ e uma sequência $\left(x_{k}\right)_{k \geq n}$ em $X$ tal que $\forall k \geq n$, $x_{k} \in \operatorname{spt} \mu_{k}$ e $x_{k} \rightarrow x$.

Proposição 2.90 (convergência fraca-estrela e variação total, parte I, [5], [7]). Sejam X espaço métrico separável localmente compacto e $\left(\mu_{k}\right)_{k \in \mathbb{N}}$ uma sequência em $\mathrm{C}_{\mathrm{c}}\left(X, \mathbb{R}^{n}\right)^{*}$ fraca-estrela convergente para $\mu \in \mathrm{C}_{\mathrm{c}}\left(X, \mathbb{R}^{n}\right)^{*}$. Então, para todo $A \subset X$ aberto, $|\mu|(A) \leq \liminf \left|\mu_{k}\right|(A)$.

Proposição 2.91 (convergência fraca-estrela e variação total, parte II, [5], [7]). Sejam X espaço métrico separável localmente compacto e $\left(\mu_{k}\right)_{k \in \mathbb{N}}$ uma sequência em $\mathrm{C}_{\mathrm{c}}\left(X, \mathbb{R}^{n}\right)^{*}$ fraca-estrela convergente para $\mu \in \mathrm{C}_{\mathrm{c}}\left(X, \mathbb{R}^{n}\right)^{*}$.

i) Se $\nu$ é uma medida de Radon positiva em $X$ e $\left|\mu_{k}\right| \stackrel{*}{\rightarrow} \nu$, então $\forall E \subset X,|\mu|(E) \leq \nu(E)$. Além disso, se $E \in \mathscr{B}_{X}$ é relativamente compacto e $\nu(\partial E)=0$, então $\mu_{k}(E) \rightarrow \mu(E)$.

ii) $S e\left|\mu_{k}\right|(X) \rightarrow|\mu|(X)<\infty$, então $\left|\mu_{k}\right| \stackrel{* f}{\rightarrow}|\mu|$.

Teorema 2.92 (de De La Vallée Poussin, [7]). Sejam X um espaço métrico separável localmente compacto e $\left(\mu_{k}\right)_{k \in \mathbb{N}}$ uma sequência de medidas de Radon finitas em $X$ a valores em $\mathbb{R}^{n}$ tal que $\sup \left\{\left|\mu_{k}\right|(X) \mid k \in \mathbb{N}\right\}<\infty$. Então existe uma medida de Radon finita $\mu$ em $X$ a valores em $\mathbb{R}^{n} e$ uma subsequência $\left(\mu_{k_{j}}\right)_{j \in \mathbb{N}}$ de $\left(\mu_{k}\right)_{k \in \mathbb{N}}$ tal que $\mu_{k_{j}} \stackrel{* \mathrm{f}}{\rightarrow} \mu$. Além disso, $|\mu|(X) \leq \liminf \left|\mu_{k_{j}}\right|(X)$.

Corolário 2.93 ([12], [5], [7]). Sejam X um espaço métrico separável localmente compacto e $\left(\mu_{k}\right)_{k \in \mathbb{N}}$ uma sequência de medidas de Radon em $X$ a valores em $\mathbb{R}^{n}$ tal que, para todo $K \subset X$ compacto, $\sup \left\{\left|\mu_{k}\right|(K) \mid k \in \mathbb{N}\right\}<\infty$. Então existe uma medida de Radon $\mu$ em $X$ a valores em $\mathbb{R}^{n}$ e uma subsequência $\left(\mu_{k_{j}}\right)_{j \in \mathbb{N}}$ de $\left(\mu_{k}\right)_{k \in \mathbb{N}}$ tal que $\mu_{k_{j}} \stackrel{*}{\rightarrow} \mu$. 


\subsection{Algumas Propriedades de Aplicações Lipschitz}

\subsubsection{Extensões}

Teorema 2.94 (lemma de McShane, [12], [5], [9], [7]). Sejam $A \subset \mathbb{R}^{n}$ e $f: A \rightarrow \mathbb{R}$ uma aplicação Lipschitz. Defina $F: \mathbb{R}^{n} \rightarrow \mathbb{R}$ por:

$$
F(x):=\inf \{f(a)+\operatorname{Lip} f \cdot\|x-a\| \mid a \in A\} .
$$

Então $F$ é uma aplicação Lipschitz que estende $f$ e $\operatorname{Lip} F=\operatorname{Lip} f$.

Teorema 2.95 (teorema de Kirszbraun, [5], [9]). Sejam $A \subset \mathbb{R}^{n}$ e $f: A \rightarrow \mathbb{R}^{m}$ uma aplicação Lipschitz. Então existe uma extensão Lipschitz $F: \mathbb{R}^{n} \rightarrow \mathbb{R}^{m}$ de $f$ tal que Lip $F=\operatorname{Lip} f$.

\subsubsection{Diferenciabilidade}

Sejam $U \subset \mathbb{R}^{n}$ aberto e $f: U \rightarrow \mathbb{R}$. Recorde que $f$ diferenciável em $x_{0} \in U$ no sentido de Fréchet se existir $A \in \mathrm{L}\left(\mathbb{R}^{n}, \mathbb{R}\right)$ tal que

$$
\lim _{h \rightarrow 0} \frac{f\left(x_{0}+h\right)-f\left(x_{0}\right)-A \cdot h}{\|h\|}=0 .
$$

Em caso afirmativo, $f$ admite derivadas parciais de primeira ordem em $x_{0}$ e a transformação linear $A$ satisfazendo as condições acima é única e dada por $\left\langle\nabla f\left(x_{0}\right), \cdot\right\rangle: \mathbb{R}^{n} \rightarrow \mathbb{R} ; A$ é chamada derivada de Fréchet de $f$ em $x_{0}$ e denotada por $\mathrm{D} f\left(x_{0}\right)$.

Equivalentemente, $f$ é diferenciável no sentido de Fréchet em $x_{0}$ se satisfizer a condição enunciada na seguinte proposição:

Proposição 2.96 (uma caracterização da diferenciabilidade no sentido de Fréchet). Sejam $U \subset \mathbb{R}^{n}$ aberto e $f: U \rightarrow \mathbb{R}$. Então $f$ é diferenciável em $x_{0} \in U$ se, e somente se, existem $A \in \mathrm{L}\left(\mathbb{R}^{n}, \mathbb{R}\right)$ e $r>0$ tais que

$$
\lim _{t \rightarrow 0^{+}} \frac{f\left(x_{0}+t v\right)-f\left(x_{0}\right)}{t}=A \cdot v
$$

uniformemente em $v \in r \mathbb{S}^{n-1}$. Em caso afirmativo, $A=\mathrm{D} f\left(x_{0}\right)$.

Demonstração. Para $r>0$ fixo, tem-se, dados $A \in \mathrm{L}\left(\mathbb{R}^{n}, \mathbb{R}\right), t>0$ e $v \in r \mathbb{S}^{n-1}$ tal que $x_{0}+t v \in U$,

$$
\frac{f\left(x_{0}+t v\right)-f\left(x_{0}\right)}{t}-A \cdot v=\|v\| \frac{f\left(x_{0}+t v\right)-f\left(x_{0}\right)-A \cdot t v}{\|t v\|}
$$

e a tese decorre imediatamente da última igualdade.

Teorema 2.97 (teorema de Rademacher, [12], [9], [7]). Seja $f: \mathbb{R}^{n} \rightarrow \mathbb{R}$ Lipschitz. Então $f$ é diferenciável no sentido de Fréchet $\mathcal{L}^{n}$-quase sempre. Além disso, $D_{f}:=\left\{x \in \mathbb{R}^{n} \mid f\right.$ derivável em $\left.x\right\}$ é boreliano, e $\mathrm{D} f: D_{f} \rightarrow \mathrm{L}\left(\mathbb{R}^{n}, \mathbb{R}\right)$ é boreliana.

Corolário 2.98. Sejam $\Omega \subset \mathbb{R}^{n}$ aberto e $f: \Omega \rightarrow \mathbb{R}^{m}$ localmente Lipschitz. Então $f$ é $\left.\mathcal{L}^{n}\right|_{\Omega^{-}}$.s.s. derivável no sentido de Fréchet, o conjunto dos pontos onde $f$ é derivável é boreliano e a derivada de $f$ é boreliana.

\section{Corolário 2.99.}

i) Sejam $f: \mathbb{R}^{n} \rightarrow \mathbb{R}^{m}$ localmente Lipschitz e $Z_{f}:=\left\{x \in \mathbb{R}^{n} \mid f(x)=0\right\}$. Então $\operatorname{D} f(x)=0$ para $\mathcal{L}^{n}$-q.t. $x \in Z_{f}$.

ii) Sejam $f, g: \mathbb{R}^{n} \rightarrow \mathbb{R}^{n}$ localmente Lipschitz e $Y:=\left\{x \in \mathbb{R}^{n} \mid g(f(x))=x\right\}$. Então $\mathrm{D} g(f(x)) \circ$ $\mathrm{D} f(x)=\operatorname{id}_{\mathbb{R}^{n}}$ para $\mathcal{L}^{n}$-q.t. $x \in Y$. 


\subsection{Jacobianos}

Definição 2.100. Sejam V e W espaços de Hilbert de dimensão finita.

i) Uma aplicação linear $O: \mathrm{V} \rightarrow \mathrm{W}$ diz-se ortogonal se $\forall x, y \in \mathrm{V},\langle O \cdot x, O \cdot y\rangle=\langle x, y\rangle$. Denotamos o conjunto das aplicações ortogonais $\mathrm{V} \rightarrow \mathrm{W}$ por $\mathrm{O}(\mathrm{V}, \mathrm{W})$; abreviamos $\mathrm{O}(n, m):=\mathrm{O}\left(\mathbb{R}^{n}, \mathbb{R}^{m}\right)$ e $\mathrm{O}(n):=\mathrm{O}(n, n)$.

ii) Seja $T: \mathrm{V} \rightarrow \mathrm{W}$ uma aplicação linear. Denotamos por $T^{*}$ a adjunta de $T$ com respeito aos produtos internos em $\mathrm{V}$ e $\mathrm{W}$, i.e. a única aplicação linear tal que $\forall x \in \mathrm{V}, \forall y \in \mathrm{W},\left\langle x, T^{*} \cdot y\right\rangle=$ $\langle T \cdot x, y\rangle$. Se $\mathrm{V}=\mathrm{W}$ e $T=T^{*}$, dizemos que $T$ é autoadjunta ou simétrica. Denotamos por $\operatorname{Sym}(V)$ o conjunto das aplicações lineares simétricas em $\mathrm{L}(\mathrm{V})$; abreviamos $\operatorname{Sym}(n):=\operatorname{Sym}\left(\mathbb{R}^{n}\right)$.

iii) Dizemos que uma aplicação linear $T: \bigvee \rightarrow \bigvee$ é positiva se for simétrica e $\forall x \in \mathrm{V},\langle T \cdot x, x\rangle \geq 0$.

Note que $\mathrm{O}(\mathrm{V}, \mathrm{W})=\emptyset$ se $\operatorname{dim} \mathrm{V}>\operatorname{dim} \mathrm{W}$.

Teorema 2.101 (decomposição polar, [12], [7]). Sejam $\mathrm{V} e \mathrm{~W}$ espaços de Hilbert de dimensão finita e $L: \mathrm{V} \rightarrow \mathrm{W}$ uma aplicação linear.

i) Se $\operatorname{dim} \mathrm{V} \leq \operatorname{dim} \mathrm{W}$, então existe $S \in \operatorname{Sym}(\mathrm{V})$ positiva e $O \in \mathrm{O}(\mathrm{V}, \mathrm{W})$ tais que

$$
L=O \circ S \text {. }
$$

Além disso, na decomposição acima, $S \in \mathrm{Sym}(\mathrm{V})$ positiva é única, e $O \in \mathrm{O}(\mathrm{V}, \mathrm{W})$ também o é se $L$ for injetiva.

ii) Se $\operatorname{dim} \mathrm{V} \geq \operatorname{dim} \mathrm{W}$, então existe $S \in \operatorname{Sym}(\mathrm{W})$ positiva e $O \in \mathrm{O}(\mathrm{W}, \mathrm{V})$ tais que

$$
L=S \circ O^{*} .
$$

Além disso, na decomposição acima, $S \in \mathrm{Sym}(\mathrm{W})$ positiva é única, e $O \in \mathrm{O}(\mathrm{W}, \mathrm{V})$ também o é se $L$ for sobrejetiva.

Definição 2.102 (jacobiano de uma aplicação linear). Sejam $\vee$ e W espaços de Hilbert de dimensão finita e $L \in \mathrm{L}(\mathrm{V}, \mathrm{W})$, com decomposição polar $O \circ S$ se $\operatorname{dim} \mathrm{V} \leq \operatorname{dim} \mathrm{W}$ ou $S \circ O^{*}$ se $\operatorname{dim} \mathrm{V}>\operatorname{dim} \mathrm{W}$, cf. teorema 2.101. Definimos o jacobiano de $L$ por:

$$
\llbracket L \rrbracket:=|\operatorname{det} S| .
$$

Observação 2.103.

1) Note que $\llbracket L \rrbracket$ está bem definido, pela unicidade de $S$ na decomposição polar.

2) Claramente,

$$
\llbracket L \rrbracket=\llbracket L^{*} \rrbracket= \begin{cases}\sqrt{\operatorname{det} L^{*} L} & \text { se } \operatorname{dim} \mathrm{V} \leq \operatorname{dim} \mathrm{W} \\ \sqrt{\operatorname{det} L L^{*}} & \text { se } \operatorname{dim} \mathrm{V} \geq \operatorname{dim} \mathrm{W}\end{cases}
$$

Teorema 2.104 (fórmula de Binet-Cauchy, [12], [7]). Sejam $\mathrm{V}$ e W espaços de Hilbert de dimensão finita com $n=\operatorname{dim} \mathrm{V} \leq \operatorname{dim} \mathrm{W}=m$. Se $L \in \mathrm{L}(\mathrm{V}, \mathrm{W})$, então

$$
\llbracket L \rrbracket=\sqrt{\sum_{B \in \mu(m, n)}(\operatorname{det} B)^{2}},
$$

onde $\mu(m, n)$ é um conjunto de $n \times n$ menores em alguma representação matricial de $L$ com respeito a bases ortonormais em $\mathrm{V} e \mathrm{~W}$.

Proposição 2.105. Seja $T \in \mathrm{L}\left(\mathbb{R}^{n}, \mathbb{R}^{m}\right)$, com $n \leq m$. 
i) Se $R \in \mathrm{L}\left(\mathbb{R}^{n}\right)$, então $\llbracket T \circ R \rrbracket=\llbracket T \rrbracket \llbracket R \rrbracket$.

ii) $\llbracket T \rrbracket \leq\|T\|^{n}$, onde $\|\cdot\|$ é a norma de operador em $\mathrm{L}\left(\mathbb{R}^{n}, \mathbb{R}^{m}\right)$. Se $T$ é $1-1$, então $\left\|T^{-1}\right\|^{-n} \leq$ $\llbracket T \rrbracket \leq\|T\|^{n}$.

Demonstração.

i)

$$
\begin{aligned}
\llbracket T \circ R \rrbracket^{2} & =\operatorname{det}\left(R^{*} T^{*} T R\right)=\operatorname{det} R^{*} \cdot \operatorname{det}\left(T^{*} T\right) \cdot \operatorname{det} R= \\
& =(\operatorname{det} R)^{2} \cdot \operatorname{det}\left(T^{*} T\right)=\llbracket T \rrbracket^{2} \llbracket R \rrbracket^{2} .
\end{aligned}
$$

ii) Para toda aplicação linear $A: \mathbb{R}^{n} \rightarrow \mathbb{R}^{n}$, segue como corolário da desigualdade clássica de Hadamard que $|\operatorname{det} A| \leq\|A\|^{n}$. Portanto,

$$
\begin{aligned}
\llbracket T \rrbracket & =\sqrt{\operatorname{det} T^{*} T} \leq \\
& \leq \sqrt{\left\|T^{*} T\right\|^{n}} \leq \sqrt{\left(\left\|T^{*}\right\|\|T\|\right)^{n}}= \\
& =\sqrt{\|T\|^{2 n}}=\|T\|^{n} .
\end{aligned}
$$

Se $n=m$ e $T \in \mathrm{GL}\left(\mathbb{R}^{n}\right)$, segue do item anterior que $\llbracket T \rrbracket \cdot \llbracket T^{-1} \rrbracket=\llbracket \operatorname{id}_{\mathbb{R}^{n}} \rrbracket=1$. Por outro lado, pela desigualdade já provada, $\llbracket T^{-1} \rrbracket \leq\left\|T^{-1}\right\|^{n}$; portanto, $\llbracket T \rrbracket=\llbracket T^{-1} \rrbracket^{-1} \geq\left\|T^{-1}\right\|^{-n}$.

No caso geral, para $T$ 1-1, tomamos uma isometria linear $P: \operatorname{Im} T \rightarrow \mathbb{R}^{n}$. Se $T=O \circ S$ é a decomposição polar de $T$, então $P \circ T=(P \circ O) \circ S$ é a decomposição polar de $P \circ T$, de modo que $\llbracket T \rrbracket=|\operatorname{det} S|=\llbracket P \circ T \rrbracket$. Por outro lado, é claro que $\|P \circ T\|=\|T\|$ e $\left\|(P \circ T)^{-1}\right\|=\left\|T^{-1}\right\|$. Como, pelo caso já provado, $\left\|(P \circ T)^{-1}\right\|^{-n} \leq \llbracket P \circ T \rrbracket \leq\|P \circ T\|^{n}$, conclui-se que $\left\|T^{-1}\right\|^{-n} \leq$ $\llbracket T \rrbracket \leq\|T\|^{n}$.

Definição 2.106 (jacobiano de aplicações Lipschitz). Seja $f: \mathbb{R}^{n} \rightarrow \mathbb{R}^{m}$ Lipschitz. Segue do teorema de Rademacher 2.97 (aplicado componente a componente) que $f$ é diferenciável no complementar de um boreliano $\mathcal{L}^{n}$-nulo e $x \mapsto \mathrm{D} f(x)$ is boreliana, definida $\mathcal{L}^{n}$-quase sempre.

Definimos, em cada ponto $x$ onde $f$ é diferenciável, o jacobiano de $f$ em $x$,

$$
\mathrm{J} f(x):=\llbracket \mathrm{D} f(x) \rrbracket .
$$

Portanto, J $f$ é uma aplicação boreliana definida no complementar de um boreliano de $\mathbb{R}^{n}$ de medida de Lebesgue nula. 


\section{Capítulo 3}

\section{Fórmulas da Área e Coárea em Espaços Euclidianos}

Neste capítulo, serão provadas versões das fórmulas da área e da coárea para aplicações Lipschitz em espaços euclidianos, respectivamente, nos teoremas 3.9 e 3.23. Estas fórmulas generalizam dois teoremas clássicos: a fórmula da área é uma generalização do teorema de mudança de variáveis para integrais múltiplas; a fórmula da coárea é uma generalização do teorema de Fubini-Tonelli.

\subsection{Linearização de Aplicações Lipschitz}

Inicialmente, desenvolveremos uma técnica, devida a Federer, para se "linearizar" aplicações Lipschitz. Esta técnica tem importância em si e, além de ser o ponto basilar a partir do qual serão demonstradas as versões das fórmulas da área e da coárea em espaços euclidianos, também será usada no próximo capítulo para se demonstrar um teorema de estrutura para conjuntos retificáveis.

Notação. Fixemos a notação abaixo, dados $n, m \in \mathbb{N}$ e $f: \mathbb{R}^{n} \rightarrow \mathbb{R}^{m}$ Lipschitz. Recorde que, conforme a notação fixada em 2.106, para todo $x \in \mathbb{R}^{n}$ onde $f$ for derivável, J $f(x)$ denota o jacobiano de $f$ em $x$.

1) $D_{f}:=\left\{x \in \mathbb{R}^{n} \mid \exists \mathrm{D} f(x)\right\}$;

2) $J_{f}^{+}:=\left\{x \in D_{f} \mid \mathrm{J} f(x)>0\right\}$;

3) $J_{f}^{0}:=\left\{x \in D_{f} \mid \mathrm{J} f(x)=0\right\}$.

Com a notação acima, o teorema de Rademacher 2.97 garante que $\mathbb{R}^{n} \backslash D_{f}$ é $\mathcal{L}^{n}$-nulo, $D_{f}$ é boreliano e $\mathrm{D} f: D_{f} \rightarrow \mathrm{L}\left(\mathbb{R}^{n}, \mathbb{R}^{m}\right)$ é boreliana. Também já vimos em 2.106 que J $f: D_{f} \rightarrow \mathbb{R}$ é boreliana; logo, $J_{f}^{+}$e $J_{f}^{0}$ também são borelianos.

Definição 3.1. Sejam $f: \mathbb{R}^{n} \rightarrow \mathbb{R}^{m}$ uma aplicação Lipschitz com $n \leq m$ e $t>1$. Dizemos que o par $(E, S)$ é uma $t$-linearização para $f$ se $E \in \mathscr{B}_{\mathbb{R}^{n}}$ e $S \in \operatorname{Sym}(n) \cap \operatorname{GL}\left(\mathbb{R}^{n}\right)$ satisfazem:

i) $\forall x \in E, f$ é diferenciável em $x$ e J $f(x)>0$;

ii) $\forall x, y \in E, t^{-1}\|S \cdot x-S \cdot y\| \leq\|f(x)-f(y)\| \leq t\|S \cdot x-S \cdot y\|$;

iii) $\forall x \in E, \forall v \in \mathbb{R}^{n}, t^{-1}\|S \cdot v\| \leq\|\mathrm{D} f(x) \cdot v\| \leq t\|S \cdot v\|$.

Proposição 3.2. Sejam $f: \mathbb{R}^{n} \rightarrow \mathbb{R}^{m}$ uma aplicação Lipschitz com $n \leq m, t>1$, e $E \in \mathscr{B}_{\mathbb{R}^{n}}$ satisfazendo o item i) da definição 3.1. Então, dado $S \in \operatorname{Sym}(n) \cap \operatorname{GL}\left(\mathbb{R}^{n}\right)$, o par $(E, S)$ é uma t-linearização para $f$ se, e somente se, $\left.f\right|_{E}$ é 1-1 com inversa Lipschitz e satisfaz:

ii') $\left.\operatorname{Lip} f\right|_{E} \circ S^{-1} \leq t e \operatorname{Lip} S \circ\left(\left.f\right|_{E}\right)^{-1} \leq t ;$

iii') $\forall x \in E,\left\|\mathrm{D} f(x) \circ S^{-1}\right\| \leq t e\left\|S \circ \mathrm{D} f(x)^{-1}\right\| \leq t$. 
Demonstração. Se $(E, S)$ for uma $t$-linearização para $f$, tem-se:

1) $\left.f\right|_{E}$ é 1-1 em vista da primeira desigualdade em ii);

2) $\left.f\right|_{E} \circ S^{-1}$ é Lipschitz, com Lip $\left.f\right|_{E} \circ S^{-1} \leq t$, em vista da segunda desigualdade em ii) com $S^{-1}\left(x^{\prime}\right)$ no lugar de $x$ e $S^{-1}\left(y^{\prime}\right)$ no lugar de $y$;

3) $S \circ\left(\left.f\right|_{E}\right)^{-1}$ é Lipschitz, com Lip $S \circ\left(\left.f\right|_{E}\right)^{-1} \leq t$, em vista da primeira desigualdade em ii) com $f^{-1}\left(x^{\prime}\right)$ no lugar de $x$ e $f^{-1}\left(y^{\prime}\right)$ no lugar de $y$;

4) analogamente, a segunda desigualdade em iii) implica $\left\|\mathrm{D} f(x) \circ S^{-1}\right\| \leq t$ e a primeira desigualdade em iii) implica $\left\|S \circ \mathrm{D} f(x)^{-1}\right\| \leq t$;

5) como $S^{-1}$ e $S \circ\left(\left.f\right|_{E}\right)^{-1}$ são ambas Lipschitz, $\left(\left.f\right|_{E}\right)^{-1}=S^{-1} \circ\left(S \circ\left(\left.f\right|_{E}\right)^{-1}\right)$ também o é.

Assim, provamos que $\left.f\right|_{E}$ é 1-1 com inversa Lipschitz e satisfaz as condições ii') e iii').

Por argumento análogo, se $\left.f\right|_{E}$ é 1-1 com inversa Lipschitz, então as condições ii') e iii') implicam ii) e iii), respectivamente, na definição 3.1 , o que prova a implicação contrária.

Corolário 3.3. Sejam $f: \mathbb{R}^{n} \rightarrow \mathbb{R}^{m}$ uma aplicação Lipschitz com $n \leq m, t>1$ e $(E, S)$ uma t-linearização para $f$. Então $\forall x \in E$,

$$
t^{-n}|\operatorname{det} S| \leq \mathrm{J} f(x) \leq t^{n}|\operatorname{det} S|
$$

Demonstração. Para todo $x \in E$, tem-se:

$$
\begin{aligned}
\mathrm{J} f(x) & =\llbracket \mathrm{D} f(x) \rrbracket\left|\operatorname{det} S^{-1}\right||\operatorname{det} S| \stackrel{2.105 \mathrm{i})}{=} \\
& =\llbracket \mathrm{D} f(x) \circ S^{-1} \rrbracket|\operatorname{det} S| .
\end{aligned}
$$

Portanto, a tese segue da proposição 2.105.ii), com $\mathrm{D} f(x) \circ S^{-1}$ no lugar de $T$ e da proposição 3.2.iii').

Teorema 3.4 (Linearização Lipschitz, [9]). Sejam $f: \mathbb{R}^{n} \rightarrow \mathbb{R}^{m}$ um aplicação Lipschitz com $n \leq m$ e $t>1$. Então existe uma família disjunta enumerável $\left(E_{k}\right)_{k \in \mathbb{N}}$ em $\mathscr{B}_{\mathbb{R}^{n}}$ tal que $J_{f}^{+}=\dot{\cup}_{k \in \mathbb{N}} E_{k}$ e, $\forall k \in \mathbb{N}$, existe $S_{k} \in \operatorname{Sym}(n) \cap \mathrm{GL}\left(\mathbb{R}^{n}\right)$ tal que $\left(E_{k}, S_{k}\right)$ é uma t-linearização para $f$.

Demonstração. Fixe $\epsilon>0$ talque $t^{-1}+\epsilon<1<t-\epsilon$. Sejam $\mathcal{S}$ um subconjunto enumerável denso de $\operatorname{Sym}(n) \cap \mathrm{GL}\left(\mathbb{R}^{n}\right)$ e $\mathcal{G}$ a um subconjunto enumerável denso de $J_{f}^{+}$. Para todo $S \in \mathcal{S}, k \in \mathbb{N}$ e $c \in \mathcal{G}$, definimos $E(S, k, c):=\mathbb{B}\left(c, \frac{1}{2 k}\right) \cap F(S, k)$, onde $F(S, k) \subset J_{f}^{+}$é o conjunto de todos os $x \in J_{f}^{+}$tais que:

F1) $\forall v \in \mathbb{R}^{n},\left(t^{-1}+\epsilon\right)\|S \cdot v\| \leq\|\mathrm{D} f(x) \cdot v\| \leq(t-\epsilon)\|S \cdot v\|$;

F2) $\forall v \in \mathbb{R}^{n}$ tal que $\|v\| \leq k^{-1},\|f(x+v)-f(x)-\mathrm{D} f(x) \cdot v\| \leq \epsilon\|S \cdot v\|$.

Como D $f$ é boreliana, é claro que $F(S, k) \in \mathscr{B}_{\mathbb{R}^{n}}, \operatorname{logo} E(S, k, c) \in \mathscr{B}_{\mathbb{R}^{n}}$. Além disso,

1) Para todo $S \in \mathcal{S}, k \in \mathbb{N}$ e $c \in \mathcal{G}, \forall x, y \in E(S, k, c)$,

$$
\begin{aligned}
& \|f(y)-f(x)\| \stackrel{F 2)}{\leq}\|\mathrm{D} f(x) \cdot(y-x)\|+\epsilon\|S \cdot(y-x)\| \stackrel{F 1)}{\leq} t\|S \cdot(y-x)\|, \\
& \|f(y)-f(x)\| \stackrel{F 2)}{\geq}\|\mathrm{D} f(x) \cdot(y-x)\|-\epsilon\|S \cdot(y-x)\| \stackrel{F 1)}{\geq} t^{-1}\|S \cdot(y-x)\| .
\end{aligned}
$$

Portanto, a condição ii) na definição 3.1 é satisfeita para o par $(E(S, k, c), S)$. Além disso, em vista de F1), a condição iii) na mesma definição é trivialmente satisfeita, de modo que $(E(S, k, c), S)$ é uma $t$-linearização para $f$. 
2) Afirmamos que $J_{f}^{+}$é a união da família enumerável $\{E(S, k, c) \mid S \in \mathcal{S}, k \in \mathbb{N}, c \in \mathcal{G}\}$. Uma vez provada tal afirmação, reenumeramos esta família como $\left(\hat{E}_{k}\right)_{k \in \mathbb{N}}$ e tomamos a sequência disjunta $\left(E_{k}\right)_{k \in \mathbb{N}}$ dada por $E_{k}:=\hat{E}_{k} \backslash \cup_{i=1}^{k-1} \hat{E}_{i}$, o que fornece a tese em vista do item anterior.

Para provar a afirmação, fixe $x \in J_{f}^{+}$e seja $\mathrm{D} f(x)=P_{x} \circ S_{x}$ a decomposição polar de $\mathrm{D} f(x)$, com $P_{x} \in \mathrm{O}(n, m)$ e $S_{x} \in \operatorname{Sym}(n)$. Note que, como $\mathrm{D} f(x)$ é $1-1, S_{x}$ também o é, i.e. $S_{x} \in \mathrm{GL}\left(\mathbb{R}^{n}\right)$. Além disso, como $\mathcal{S}$ é denso em $\operatorname{Sym}(n) \cap \mathrm{GL}\left(\mathbb{R}^{n}\right)$, podemos tomar uma sequência $\left(S_{i}\right)_{i \in \mathbb{N}}$ em $\mathcal{S}$ convergente para $S_{x}$; portanto, por continuidade, $S_{x} \circ S_{i}^{-1} \rightarrow \mathrm{id}_{\mathbb{R}^{n}}$ e $S_{i} \circ S_{x}^{-1} \rightarrow \mathrm{id}_{\mathbb{R}^{n}}$. Tomando $i$ suficientemente grande, conclui-se que existe $S \in \mathcal{S}$ tal que

$$
\left\|S_{x} \circ S^{-1}\right\| \leq t-\epsilon \quad \text { and } \quad\left\|S \circ S_{x}^{-1}\right\| \leq\left(t^{-1}+\epsilon\right)^{-1} .
$$

Daí segue que, $\forall v \in \mathbb{R}^{n}$,

$$
\begin{aligned}
\|\mathrm{D} f(x) \cdot v\| & =\left\|P_{x} \cdot S_{x} \cdot v\right\|=\left\|S_{x} \cdot v\right\|=\left\|\left(S_{x} S^{-1}\right) S \cdot v\right\| \leq \\
& \leq\left\|S_{x} S^{-1}\right\|\|S \cdot v\| \leq(t-\epsilon)\|S \cdot v\|, \\
\left(t^{-1}+\epsilon\right)\|S \cdot v\| & =\left(t^{-1}+\epsilon\right)\left\|\left(S S_{x}^{-1}\right) S_{x} \cdot v\right\| \leq \\
& \leq\left(t^{-1}+\epsilon\right)\left\|S S_{x}^{-1}\right\|\left\|S_{x} \cdot v\right\| \leq\left\|S_{x} \cdot v\right\|=\|\mathrm{D} f(x) \cdot v\| .
\end{aligned}
$$

Ou seja, F1) é satisfeita. Além disso, pela diferenciabilidade de $f$ em $x$, existe $R(x, \cdot): \mathbb{R}^{n} \rightarrow$ $[0, \infty)$ contínua e nula em $v=0$, tal que $\forall v \in \mathbb{R}^{n}$ :

$$
\begin{aligned}
\|f(x+v)-f(x)-\mathrm{D} f(x) \cdot v\| & =R(x, v)\|v\|=R(x, v)\left\|S^{-1} S \cdot v\right\| \leq \\
& \leq R(x, v)\left\|S^{-1}\right\|\|S \cdot v\| .
\end{aligned}
$$

Como $\lim _{v \rightarrow 0} R(x, v)=R(x, 0)=0$, podemos tomar $k \in \mathbb{N}$ suficientemente grande de modo a garantir $R(x, v)\left\|S^{-1}\right\| \leq \epsilon$ para $\|v\| \leq k^{-1}$, logo F2) é satisfeita para esta escolha de $k$. Conclui-se, pois, que $x \in F(S, k)$. Finalmente, como $\mathcal{G}$ é denso em $J_{f}^{+}$, existe $c \in \mathcal{G}$ tal que $c \in \mathbb{U}\left(x, \frac{1}{2 k}\right) \Leftrightarrow x \in \mathbb{U}\left(c, \frac{1}{2 k}\right), \log \mathrm{o} x \in E(S, k, c)=\mathbb{B}\left(c, \frac{1}{2 k}\right) \cap F(S, k)$, o que conclui a prova da afirmação.

\subsection{Fórmula da Área em Espaços Euclidianos}

Inicialmente, recordemos o teorema de mudança de variáveis linear para a integral de Lebesgue. Remetemos a demonstração para textos clássicos em Medida e Integração, como [6] ou [18]; essencialmente, é uma consequência da invariância da medida de Lebesgue unidimensional por translações e da sua homogeneidade com respeito a homotetias.

Teorema 3.5 (mudança de variáveis linear para a integral de Lebesgue). Seja $T \in G L(n, \mathbb{R})$.

i) Se $A \in \sigma\left(\mathcal{L}^{n}\right)$, então $T \cdot A \in \sigma\left(\mathcal{L}^{n}\right)$ e $\mathcal{L}^{n}(T \cdot A)=|\operatorname{det} T| \mathcal{L}^{n}(A)$.

ii) Se $f: \mathbb{R}^{n} \rightarrow \mathbb{R}$ é Lebesgue mensurável, também ó é $f \circ T$ e, se $f \geq 0$ ou $f \in L^{1}$,

$$
\int f \mathrm{~d} \mathcal{L}^{n}=\int f \circ T|\operatorname{det} T| \mathrm{d} \mathcal{L}^{n}
$$

Usaremos o teorema acima na prova das versões lineares da fórmula da área, abaixo, e da fórmula da coárea, em 3.17.

Lema 3.6 (Formula da Área, caso linear). Se $L: \mathbb{R}^{n} \rightarrow \mathbb{R}^{m}$ é linear e $n \leq m$, então, $\forall A \subset \mathbb{R}^{n}$,

$$
\mathcal{H}^{n}(L(A))=\llbracket L \rrbracket \mathcal{L}^{n}(A)
$$


Demonstração. Seja $L=O \circ S$ a decomposição polar de $L$, cf. teorema 2.101, com $S \in \operatorname{Sym}(n)$ positiva e $O \in \mathrm{O}(n, m)$. Então $\llbracket L \rrbracket=|\operatorname{det} S|$. Tem-se:

1) Se $\llbracket L \rrbracket=0$, então $\operatorname{det} S=0$, de modo que $\operatorname{dim} \operatorname{Im} L=\operatorname{dim} \operatorname{Im} S \leq n-1$. Portanto, segue do corolário 2.56 que $\mathcal{H}$-dim $\operatorname{Im} L \leq n-1$, logo $\mathcal{H}^{n}\left(L\left(\mathbb{R}^{n}\right)\right)=0$, donde, $\forall A \subset \mathbb{R}^{n}, \mathcal{H}^{n}(L(A))=0$ e ambos os membros de (3.2) se anulam.

2) Se $\llbracket L \rrbracket>0$, então det $S>0$ e $O: \mathbb{R}^{n} \rightarrow \mathbb{R}^{m}$ é uma isometria linear. Daí, segue da proposição 2.49.ii) que, para todo $A \subset \mathbb{R}^{n}$ boreliano:

$$
\begin{aligned}
\frac{\mathcal{H}^{n}(L(A))}{\mathcal{L}^{n}(A)} & =\frac{\mathcal{H}^{n}(O \circ S(A))}{\mathcal{L}^{n}(A)}= \\
& =\frac{\mathcal{H}^{n}(S(A))}{\mathcal{L}^{n}(A)} \stackrel{2.54}{=} \\
& =\frac{\mathcal{L}^{n}(S(A))}{\mathcal{L}^{n}(A)} \stackrel{3.5 . i)}{=} \\
& =|\operatorname{det} S|=\llbracket L \rrbracket .
\end{aligned}
$$

3) Defina, $\forall A \subset \mathbb{R}^{n}, \nu(A):=\mathcal{H}^{n}(L(A))$. Afirmamos que $\nu$ é uma medida de Radon em $\mathbb{R}^{n}$. De fato, $L: \mathbb{R}^{n} \rightarrow \mathbb{R}^{m}$ é um isomorfismo linear sobre $\operatorname{Im} L$. Em particular, $L: \mathbb{R}^{n} \rightarrow \operatorname{Im} L$ é um homeomorfismo (munindo $\operatorname{Im} L$ da topologia relativa), logo a operação de pushforward $L_{\#}$ define uma bijeção entre medidas borelianas em $\mathbb{R}^{n}$ e medidas borelianas em $\operatorname{Im} L$, com inversa dada por $L^{-1} \#$; além disso, é claro que esta bijeção se restringe a uma bijeção entre medidas Borel-regulares. Como $\mathcal{H}^{n} \mid \operatorname{Im} L$ é uma medida Borel-regular (o que pode ser checado diretamente, em vista da Borel-regularidade de $\mathcal{H}^{n}$ em $\mathbb{R}^{m}$, ou a partir do fato de que o traço $\left.\mathcal{H}^{n}\right|_{\operatorname{Im} L}$ coincide com a medida de Hausdorff $m$-dimensional de $\operatorname{Im} L$ como subespaço métrico de $\mathbb{R}^{m}$, pela proposição 2.49.i), segue que $\nu=L^{-1} \#\left(\mathcal{H}^{n} \mid \operatorname{Im} L\right)$ é uma medida Borel-regular em $\mathbb{R}^{n}$. Além disso, $\forall K \subset \mathbb{R}^{n}$ compacto, segue da proposição 2.49.iii) que $\nu(K)=\mathcal{H}^{n}(L(K)) \leq$ $(\operatorname{Lip} L)^{n} \mathcal{H}^{n}(K)=(\operatorname{Lip} L)^{n} \mathcal{L}^{n}(K)<\infty$. Ou seja, $\nu$ é uma medida Borel-regular localmente finita $\mathbb{R}^{n}$, logo é de Radon por 2.24.2), o que conclui a prova da afirmação.

4) Segue de (3.3) que as medidas Borel-regulares $\nu$ e $\llbracket L \rrbracket \mathcal{L}^{n}$ coincidem em todos os borelianos; portanto, em vista da Borel-regularidade, elas coincidem em todo $A \subset \mathbb{R}^{n}$, i.e. $\mathcal{H}^{n}(L(A))=$ $\llbracket L \rrbracket \mathcal{L}^{n}(A)$, como queríamos mostrar.

Lema 3.7. Sejam $f: \mathbb{R}^{n} \rightarrow \mathbb{R}^{m}$ Lipschitz, com $n \leq m$, e $A \subset \mathbb{R}^{n} \mathcal{L}^{n}$-mensurável. Então:

i) $f(A)$ é $\mathcal{H}^{n}$-mensurável.

ii) A função $N\left(\left.f\right|_{A}\right): \mathbb{R}^{m} \rightarrow[0, \infty]$ dada por $y \mapsto \mathcal{H}^{0}\left(A \cap f^{-1}\{y\}\right)$ é $\mathcal{H}^{n}$-mensurável.

iii) $\int_{\mathbb{R}^{m}} \mathcal{H}^{0}\left(A \cap f^{-1}\{y\}\right) \mathrm{d} \mathcal{H}^{n}(y) \leq(\operatorname{Lip} f)^{n} \mathcal{L}^{n}(A)$.

Definição 3.8 (função de multiplicidade). Com a notação do lema precedente, $N\left(\left.f\right|_{A}\right): y \mapsto$ $\mathcal{H}^{0}\left(A \cap f^{-1}\{y\}\right)$ chama-se função de multiplicidade de $\left.f\right|_{A}$.

\section{Demonstração.}

i) Como $\mathcal{L}^{n}$ é $\sigma$-finita, podemos tomar uma sequência $\left(A_{k}\right)_{k \in \mathbb{N}}$ em $\sigma\left(\mathcal{L}^{n}\right)$ tal que, $\forall k \in \mathbb{N}$, $\mathcal{L}^{n}\left(A_{k}\right)<\infty$ e $\cup_{k \in \mathbb{N}} A_{k}=A$. Daí $f(A)=\cup_{k \in \mathbb{N}} f\left(A_{k}\right)$, de modo que $f(A) \in \sigma\left(\mathcal{H}^{n}\right)$ se provarmos que, $\forall k \in \mathbb{N}, f\left(A_{k}\right) \in \sigma\left(\mathcal{H}^{n}\right)$. É suficiente, pois, provar o caso em que $\mathcal{L}^{n}(A)<\infty$. Como $\mathcal{L}^{n}$ é uma medida de Radon e $\mathbb{R}^{n}$ é $\sigma$-compacto, podemos tomar (por 2.24.i) uma sequência crescente $\left(K_{i}\right)_{i \in \mathbb{N}}$ de subconjuntos compactos de $A$ tal que $\mathcal{L}^{n}\left(K_{i}\right) \rightarrow \mathcal{L}^{n}(A)$. Como $A \in \sigma\left(\mathcal{L}^{n}\right)$ e $\mathcal{L}^{n}(A)<\infty$, segue que $\mathcal{L}^{n}\left(A \backslash K_{i}\right) \rightarrow 0, \operatorname{logo} \mathcal{L}^{n}\left(A \backslash \cup_{i \in \mathbb{N}} K_{i}\right)=0$. Portanto, pela proposição 
2.49.iii), conclui-se que $\mathcal{H}^{n}\left(f\left(A \backslash \cup_{i \in \mathbb{N}} K_{i}\right)\right) \leq(\operatorname{Lip} f)^{n} \mathcal{L}^{n}\left(A \backslash \cup_{i \in \mathbb{N}} K_{i}\right)=0$. Como $\forall i \in \mathbb{N}, f\left(K_{i}\right)$ é compacto, segue $\cup_{i \in \mathbb{N}} f\left(K_{i}\right) \in \mathscr{B}_{\mathbb{R}^{m}} \subset \sigma\left(\mathcal{H}^{n}\right)$. De $f(A) \backslash \cup_{i \in \mathbb{N}} f\left(K_{i}\right) \subset f\left(A \backslash \cup_{i \in \mathbb{N}} K_{i}\right)$ concluise que $f(A) \backslash \cup_{i \in \mathbb{N}} f\left(K_{i}\right)$ é $\mathcal{H}^{n}$-nulo, i.e. $f(A)$ é a união de um boreliano com um conjunto $\mathcal{H}^{n}$-nulo, $\log$ o $f(A) \in \sigma\left(\mathcal{H}^{n}\right)$.

ii) Podemos tomar uma sequência $\left(\mathcal{F}_{i}\right)_{i \in \mathbb{N}}$ tal que

- $\forall i \in \mathbb{N}, \mathcal{F}_{i}=\left(F_{j}^{i}\right)_{j \in \mathbb{N}}$ é uma família disjunta de borelianos de $\mathbb{R}^{n} \operatorname{com}, \forall j \in \mathbb{N}$, diam $F_{j}^{i} \leq$ $1 / i$ e $\dot{\cup}_{j \in \mathbb{N}} F_{j}^{i}=\mathbb{R}^{n}$;

- $\forall i \in \mathbb{N}$, cada $F_{j}^{i+1}$ é um subconjunto de algum $F_{k}^{i}$ (de modo que cada $F_{k}^{i}$ é a união disjunta de alguns dos termos de $\left.\mathcal{F}_{i+1}\right)$.

Seja $\left(g_{i}\right)_{i \in \mathbb{N}}$ a sequência de funções $\mathbb{R}^{m} \rightarrow[0, \infty]$ definida por, $\forall i \in \mathbb{N}$,

$$
g_{i}:=\sum_{j \in \mathbb{N}} \chi_{f}\left(A \cap F_{j}^{i}\right)
$$

(a ideia é que, para cada $i \in \mathbb{N}$ e $y \in \mathbb{R}^{m}, g_{i}(y)$ é o número de termos de $\mathcal{F}_{i}$ que intersectam $A \cap f^{-1}\{y\}$; intuitivamente, $g_{i}$ cresce pontualmente para a função de multiplicidade). A tese seguirá uma vez que mostremos que cada $g_{i}$ é $\mathcal{H}^{n}$-mensurável e que $\left(g_{i}\right)_{i \in \mathbb{N}}$ cresce pontualmente para a função de multiplicidade $N\left(\left.f\right|_{A}\right)$ (o que implica a $\mathcal{H}^{n}$-mensurabilidade da última função em vista do teorema 2.31.iv). Isso será feito ao longo dos seguintes passos:

1) $\forall i, j \in \mathbb{N}, A \cap F_{j}^{i} \in \sigma\left(\mathcal{L}^{n}\right), \operatorname{logo} \chi_{f\left(A \cap F_{j}^{i}\right)}$ é $\mathcal{H}^{n}$-mensurável pela parte i). Assim, $\forall i \in \mathbb{N}$, $g_{i}:=\sum_{j \in \mathbb{N}} \chi_{f\left(A \cap F_{j}^{i}\right)}$ é $\mathcal{H}^{n}$-mensurável pelo teorema 2.31.iv).

2) $\left(g_{i}\right)_{i \in \mathbb{N}}$ é pontualmente crescente. De fato, $\forall y \in \mathbb{R}^{m}$ e $i \in \mathbb{N}$, para cada $j \in \mathbb{N}$ tal que $A \cap f^{-1}\{y\}$ corta $F_{j}^{i}$, i.e. tal que $\chi_{f\left(A \cap F_{j}^{i}\right)}(y)=1$, o fato de que $F_{j}^{i}$ é uma união de termos de $\mathcal{F}_{i+1}$ implica a existência de $k=k_{i}(j) \in \mathbb{N}$ tal que $F_{k}^{i+1} \subset F_{j}^{i}\left(\operatorname{logo} k_{i}(j) \neq k_{i}\left(j^{\prime}\right)\right.$ se $j \neq j^{\prime}$, i.e. $k_{i}$ é $\left.1-1\right)$ e $A \cap f^{-1}\{y\}$ corta $F_{k}^{i+1}$, i.e. $\chi_{f\left(A \cap F_{k}^{i+1}\right)}(y)=1$. Então, definindo $N_{i}:=\left\{j \in \mathbb{N} \mid A \cap f^{-1}\{y\} \cap F_{j}^{i} \neq \emptyset\right\}$, tem-se

$$
\begin{aligned}
g_{i}(y) & =\sum_{j \in \mathbb{N}} \chi_{f\left(A \cap F_{j}^{i}\right)}(y)=\sum_{j \in N_{i}} \chi_{f\left(A \cap F_{j}^{i}\right)}(y)= \\
& =\sum_{j \in N_{i}} \chi_{f\left(A \cap F_{k_{i}(j)}^{i+1}\right)}(y) \stackrel{k_{i} \text { is } 1-1}{\leq} \\
& \leq \sum_{j \in \mathbb{N}} \chi_{f\left(A \cap F_{j}^{i+1}\right)}(y)=g_{i+1}(y) .
\end{aligned}
$$

3) $\forall i \in \mathbb{N}, g_{i} \leq N\left(\left.f\right|_{A}\right)$. De fato, como $\mathcal{F}_{i}$ é uma família disjunta, para todo $y \in \mathbb{R}^{m}, A \cap$ $f^{-1}\{y\}=\dot{\cup}_{j \in \mathbb{N}} A \cap f^{-1}\{y\} \cap F_{j}^{i}$. Como, $\forall j \in \mathbb{N}, \mathcal{H}^{0}\left(A \cap f^{-1}\{y\} \cap F_{j}^{i}\right) \geq \chi_{f\left(A \cap F_{j}^{i}\right)}(y)$, segue que $N\left(\left.f\right|_{A}\right)(y)=\mathcal{H}^{0}\left(A \cap f^{-1}\{y\}\right)=\sum_{j \in \mathbb{N}} \mathcal{H}^{0}\left(A \cap f^{-1}\{y\} \cap F_{j}^{i}\right) \geq \sum_{j \in \mathbb{N}} \chi_{f\left(A \cap F_{j}^{i}\right)}(y)=g_{i}(y)$.

4) $\forall y \in \mathbb{R}^{m}, \forall k \in \mathbb{N}$ tal que $k \leq N\left(\left.f\right|_{A}\right)(y)$, existe $i \in \mathbb{N}$ tal que $g_{i}(y) \geq k$. De fato, como $N\left(\left.f\right|_{A}\right)(y)=\mathcal{H}^{0}\left(A \cap f^{-1}\{y\}\right) \geq k$, podemos escolher $k$ pontos distintos $x_{1}, \ldots, x_{k} \in$ $A \cap f^{-1}\{y\}$. Tome $i \in \mathbb{N}$ tal que $\left\|x_{p}-x_{q}\right\|>1 / i$ para $1 \leq p<q \leq k$. Como os termos de $\mathcal{F}_{i}$ são disjuntos e têm diâmetros $\leq 1 / i$, segue que, $\forall 1 \leq p \leq k, x_{p}$ pertence a exatamente um dos termos de $\mathcal{F}_{i}$, digamos, $F_{j(p)}^{i}$, com $p \mapsto j(p) 1-1$. Então $g_{i}(y)=\sum_{j \in \mathbb{N}} \chi_{f\left(A \cap F_{j}^{i}\right)}(y) \geq$ $\sum_{1 \leq p \leq k} \chi_{f\left(A \cap F_{j(p)}^{i}\right)}(y)=k$, como afirmado.

iii) Seja $\left(g_{i}\right)_{i \in \mathbb{N}}$ a mesma sequência de funções $\mathbb{R} \rightarrow[0, \infty]$ do item anterior, de modo que $\forall y \in \mathbb{R}^{m}$, 
$g_{i}(y) \uparrow N\left(\left.f\right|_{A}\right)(y)$. Então segue do teorema da convergência monótona que:

$$
\begin{aligned}
\int_{\mathbb{R}^{m}} N\left(f||_{A}\right)(y) \mathrm{d} \mathcal{H}^{n}(y) & =\lim _{i \rightarrow \infty} \int_{\mathbb{R}^{m}} g_{i}(y) \mathrm{d} \mathcal{H}^{n}(y)= \\
& =\lim _{i \rightarrow \infty} \sum_{j \in \mathbb{N}} \mathcal{H}^{n}\left(f\left(A \cap F_{j}^{i}\right)\right) \stackrel{2.49 . i i i)}{\leq} \\
& \leq \liminf _{i \rightarrow \infty} \sum_{j \in \mathbb{N}}(\operatorname{Lip} f)^{n} \mathcal{L}^{n}\left(A \cap F_{j}^{i}\right)= \\
& =(\operatorname{Lip} f)^{n} \mathcal{L}^{n}(A) .
\end{aligned}
$$

Teorema 3.9 (Formula da Área). Seja $f: \mathbb{R}^{n} \rightarrow \mathbb{R}^{m}$ Lipschitz, com $n \leq m$. Então, para todo $A \in \sigma\left(\mathcal{L}^{n}\right)$,

$$
\int_{A} \mathrm{~J} f \mathrm{~d} \mathcal{L}^{n}=\int_{\mathbb{R}^{m}} \mathcal{H}^{0}\left(A \cap f^{-1}\{y\}\right) \mathrm{d} \mathcal{H}^{n}(y) .
$$

Demonstração. Recorde a notação fixada no início deste capítulo em 3.1.

Se $\mathcal{L}^{n}(A)=0$, o primeiro membro se anula trivialmente, e o segundo membro se anula pelo lema 3.7.iii). Portanto, em vista do teorema de Rademacher 2.97, podemos assumir $A \subset D_{f}$.

1) Caso 1: $A \subset J_{f}^{+}$. Fixe $t>1$. Seja $\left(E_{k}\right)_{k \in \mathbb{N}}$ uma sequência em $\mathscr{B}_{\mathbb{R}^{n}}$ dada pelo teorema de linearização Lipschitz 3.4, i.e. tal que $J_{f}^{+}=\dot{\cup}_{k \in \mathbb{N}} E_{k}$ e, para cada $k \in \mathbb{N}$, existe $S_{k} \in \operatorname{Sym}(n) \cap$ $\mathrm{GL}\left(\mathbb{R}^{n}\right)$ tal que $\left(E_{k}, S_{k}\right)$ é uma $t$-linearização para $f$. Então, $\forall k \in \mathbb{N}$,

$$
\begin{aligned}
\mathcal{H}^{n}\left(f\left(A \cap E_{k}\right)\right) & =\mathcal{H}^{n}\left(\left.f\right|_{E_{k}} \circ S_{k}^{-1} \circ S_{k}\left(A \cap E_{k}\right)\right) \stackrel{2.49}{\leq} \\
& \leq\left(\left.\operatorname{Lip} f\right|_{E_{k}} \circ S_{k}^{-1}\right)^{n} \mathcal{H}^{n}\left(S_{k}\left(A \cap E_{k}\right)\right) \stackrel{\left.3.2 . i i^{\prime}\right)}{\leq} \\
& \leq t^{n} \mathcal{H}^{n}\left(S_{k}\left(A \cap E_{k}\right)\right),
\end{aligned}
$$

$\mathrm{e}$

$$
\begin{aligned}
\mathcal{H}^{n}\left(S_{k}\left(A \cap E_{k}\right)\right) & =\mathcal{H}^{n}\left(S_{k} \circ\left(\left.f\right|_{E_{k}}\right)^{-1} \circ f\left(A \cap E_{k}\right)\right) \stackrel{2.49}{\leq} \\
& \leq\left(\operatorname{Lip} S_{k} \circ\left(\left.f\right|_{E_{k}}\right)^{-1}\right)^{n} \mathcal{H}^{n}\left(f\left(A \cap E_{k}\right)\right) \stackrel{\left.3.2 . i i^{\prime}\right)}{\leq} \\
& \leq t^{n} \mathcal{H}^{n}\left(f\left(A \cap E_{k}\right)\right),
\end{aligned}
$$

Por outro lado, segue do corolário 3.3 que, $\forall k \in \mathbb{N}, \forall x \in E_{k}$,

$$
t^{-n} \llbracket S_{k} \rrbracket \leq \mathrm{J} f(x) \leq t^{n} \llbracket S_{k} \rrbracket .
$$

Portanto, $\forall k \in \mathbb{N}$ :

$$
\begin{aligned}
t^{-2 n} \mathcal{H}^{n}\left(f\left(A \cap E_{k}\right)\right) & \stackrel{(3.4)}{\leq} t^{-n} \mathcal{H}^{n}\left(S_{k}\left(A \cap E_{k}\right)\right) \stackrel{\text { lema }}{=} 3.6 \\
& =t^{-n} \llbracket S_{k} \rrbracket \mathcal{L}^{n}\left(A \cap E_{k}\right) \stackrel{(3.6)}{\leq} \\
& \leq \int_{A \cap E_{k}} \mathrm{~J} f(x) \mathrm{d} \mathcal{L}^{n}(x) \stackrel{(3.6)}{\leq} \\
& \leq t^{n} \llbracket S_{k} \rrbracket \mathcal{L}^{n}\left(A \cap E_{k}\right) \stackrel{\text { lema }}{=} 3.6 \\
& =t^{n} \mathcal{H}^{n}\left(S_{k}\left(A \cap E_{k}\right)\right) \stackrel{(3.5)}{\leq} \\
& \leq t^{2 n} \mathcal{H}^{n}\left(f\left(A \cap E_{k}\right)\right)
\end{aligned}
$$


Como, $\forall k \in \mathbb{N},\left.f\right|_{E_{k}}$ é 1-1 (pela proposição 3.2), tem-se, $\forall y \in \mathbb{R}^{m}, \mathcal{H}^{0}\left(A \cap E_{k} \cap f^{-1}\{y\}\right)=$ $\chi_{f\left(A \cap E_{k}\right)}(y)$, de modo que $\int_{\mathbb{R}^{m}} \mathcal{H}^{0}\left(A \cap E_{k} \cap f^{-1}\{y\}\right) \mathrm{d} \mathcal{H}^{n}(y)=\mathcal{H}^{n}\left(f\left(A \cap E_{k}\right)\right)$. Assim, de (3.7) e do teorema da convergência monótona, conclui-se que:

$$
\begin{aligned}
t^{-2 n} \int_{\mathbb{R}^{m}} \mathcal{H}^{0}\left(A \cap f^{-1}\{y\}\right) \mathrm{d} \mathcal{H}^{n}(y) & \stackrel{\mathrm{T}}{=} \sum_{k \in \mathbb{N}} t^{-2 n} \int_{\mathbb{R}^{m}} \mathcal{H}^{0}\left(A \cap E_{k} \cap f^{-1}\{y\}\right) \mathrm{d} \mathcal{H}^{n}(y)= \\
& =\sum_{k \in \mathbb{N}} t^{-2 n} \mathcal{H}^{n}\left(f\left(A \cap E_{k}\right)\right) \stackrel{(3.7)}{\leq} \\
& \leq \sum_{k \in \mathbb{N}} \int_{A \cap E_{k}} \mathrm{~J} f(x) \mathrm{d} \mathcal{L}^{n}(x) \stackrel{\mathrm{TCM}}{=} \int_{A} \mathrm{~J} f(x) \mathrm{d} \mathcal{L}^{n}(x) \stackrel{(3.7)}{\leq} \\
& \leq \sum_{k \in \mathbb{N}} t^{2 n} \mathcal{H}^{n}\left(f\left(A \cap E_{k}\right)\right)= \\
& =\sum_{k \in \mathbb{N}} t^{2 n} \int_{\mathbb{R}^{m}} \mathcal{H}^{0}\left(A \cap E_{k} \cap f^{-1}\{y\}\right) \mathrm{d} \mathcal{H}^{n}(y) \stackrel{\mathrm{T}}{=} \\
& =t^{2 n} \int_{\mathbb{R}^{m}} \mathcal{H}^{0}\left(A \cap f^{-1}\{y\}\right) \mathrm{d} \mathcal{H}^{n}(y) .
\end{aligned}
$$

Logo,

$$
t^{-2 n} \int_{\mathbb{R}^{m}} \mathcal{H}^{0}\left(A \cap f^{-1}\{y\}\right) \mathrm{d} \mathcal{H}^{n}(y) \leq \int_{A} \mathrm{~J} f \mathrm{~d} \mathcal{L}^{n} \leq t^{2 n} \int_{\mathbb{R}^{m}} \mathcal{H}^{0}\left(A \cap f^{-1}\{y\}\right) \mathrm{d} \mathcal{H}^{n}(y) .
$$

Tomando $t \downarrow 1$, segue

$$
\int_{\mathbb{R}^{m}} \mathcal{H}^{0}\left(A \cap f^{-1}\{y\}\right) \mathrm{d} \mathcal{H}^{n}(y)=\int_{A} \mathrm{~J} f \mathrm{~d} \mathcal{L}^{n}
$$

como afirmado.

2) Caso 2: $A \subset J_{f}^{0}$. Então $\int_{A} \mathrm{~J} f \mathrm{~d} \mathcal{L}^{n}=0$; devemos mostrar que $\int \mathcal{H}^{0}\left(A \cap f^{-1}\{y\}\right) \mathrm{d} \mathcal{H}^{n}(y)=0$. Podemos assumir que $\mathcal{L}^{n}(A)<\infty$ (o caso geral seguirá pelo teorema da convergência monótona, escrevendo $A=\dot{\cup}_{n \in \mathbb{N}} A_{n}$, com $\forall n \in \mathbb{N}, A_{n} \in \sigma\left(\mathcal{L}^{n}\right)$ e $\mathcal{L}^{n}\left(A_{n}\right)<\infty$, o que é possível pela $\sigma$-finitude da medida de Lebesgue).

Fixe $0<\epsilon<1$. Defina $g: \mathbb{R}^{n} \rightarrow \mathbb{R}^{m+n} \equiv \mathbb{R}^{m} \times \mathbb{R}^{n}$ por $g(x):=(f(x), \epsilon x)$. Então $g$ é Lipschitz 1-1 e, $\forall x \in D_{g}=D_{f}, \mathrm{D} g(x)=\left(\mathrm{D} f(x), \epsilon \operatorname{id}_{\mathbb{R}^{n}}\right) \in \mathrm{L}\left(\mathbb{R}^{n}, \mathbb{R}^{m} \times \mathbb{R}^{n}\right)$.

Afirmamos que existe $C=C(n, m, \operatorname{Lip} f)>0$ (em particular, $C$ não depende de $\epsilon$ ) tal que, $\forall x \in A, 0<\mathrm{J} g(x) \leq C \epsilon$. Assumindo tal afirmação, tem-se, denotando por $\mathrm{pr}_{1}: \mathbb{R}^{m} \times \mathbb{R}^{n} \rightarrow \mathbb{R}^{m}$ a projeção no primeiro fator:

$$
\begin{aligned}
\mathcal{H}^{n}(f(A)) & =\mathcal{H}^{n}\left(\operatorname{pr}_{1} \circ g(A)\right) \stackrel{2.49 .3)}{\leq} \\
& \leq\left(\operatorname{Lip~pr}_{1}\right)^{n} \mathcal{H}^{n}(g(A)) \stackrel{g 1-1 \mathrm{e} \stackrel{\text { Lip pr }_{1}=1}{=}}{ } \\
& =\int_{\mathbb{R}^{m+n}} \mathcal{H}^{0}\left(A \cap g^{-1}\{y\}\right) \mathrm{d} \mathcal{H}^{n}(y) \stackrel{\text { caso } 1}{=} \\
& =\int_{A} \mathrm{~J} g \mathrm{~d} \mathcal{L}^{n} \stackrel{\text { afirmação }}{\leq} \\
& \leq C \epsilon \mathcal{L}^{n}(A) .
\end{aligned}
$$

Portanto, como $0<\epsilon<1$ foi escolhido arbitrariamente e assumimos $\mathcal{L}^{n}(A)<\infty$, segue que $\mathcal{H}^{n}(f(A))=0$. Como a função de multiplicidade $N\left(\left.f\right|_{A}\right): \mathbb{R}^{n} \rightarrow \mathbb{R}, y \mapsto \mathcal{H}^{0}\left(A \cap f^{-1}\{y\}\right)$, tem suporte em $f(A)$, segue que $\int \mathcal{H}^{0}\left(A \cap f^{-1}\{y\}\right) \mathrm{d} \mathcal{H}^{n}(y)=\int_{f(A)} \mathcal{H}^{0}\left(A \cap f^{-1}\{y\}\right) \mathrm{d} \mathcal{H}^{n}(y)=0$, 
como queríamos.

Resta provar a afirmação. Como, $\forall x \in D_{g}=D_{f}, \mathrm{D} g(x)=\left(\mathrm{D} f(x), \epsilon \mathrm{id}_{\mathbb{R}^{n}}\right) \in \mathrm{L}\left(\mathbb{R}^{n}, \mathbb{R}^{m} \times \mathbb{R}^{n}\right)$, a matriz jacobiana de $\mathrm{D} g(x)$ é a matriz $(m+n) \times n$ que se escreve em blocos como:

$$
[\mathrm{D} g(x)]=\left(\begin{array}{c}
{[\mathrm{D} f(x)]} \\
\epsilon I_{n}
\end{array}\right)
$$

Pela fórmula de Binet-Cauchy 2.104, $(\mathrm{J} g(x))^{2}$ é a soma dos quadrados dos $n \times n$-menores da matriz acima. Em particular, tomando o menor correspondente às últimas $n$ linhas, conclui-se que, $\forall x \in D_{g}=D_{f}$, J $g(x) \geq \epsilon^{n}>0$. Por outro lado, para se obter um limitante superior para a referida soma:

- Note que a $i$-ésima linha da matriz $[\mathrm{D} f(x)]$ é $\nabla f^{i}(x)$, onde $f^{i}$ é a $i$-ésima componente de $f$ na base canônica de $\mathbb{R}^{m}$; a norma desta linha é, portanto, $\left\|\nabla f^{i}(x)\right\|=\left\|\mathrm{D} f^{i}(x)\right\| \leq \operatorname{Lip} f^{i} \leq$ $\operatorname{Lip} f$.

- A soma dos quadrados dos menores $n \times n$ de [D $g(x)]$ pode ser escrita como $M_{1}+M_{2}$, onde os termos em $M_{1}$ são os quadrados dos menores $n \times n$ com linhas em [D $\left.f(x)\right]$, i.e. $M_{1}=(\mathrm{J} f(x))^{2}$, e os termos em $M_{2}$ são os quadrados dos demais menores, i.e. os menores $n \times n$ que têm ao menos uma linha em $\epsilon I_{n}$. Como $\epsilon<1$ e as linhas em [D $\left.f(x)\right]$ têm normas limitadas por Lip $f$, cada menor do último tipo é limitado por $\epsilon \cdot \max \left\{1,(\operatorname{Lip} f)^{n-1}\right\}$. Como existem $\left(\begin{array}{c}m+n \\ n\end{array}\right)-\left(\begin{array}{c}m \\ n\end{array}\right)$ parcelas em $M_{2}$, tem-se $M_{2} \leq\left(\left(\begin{array}{c}m+n \\ n\end{array}\right)-\left(\begin{array}{c}m \\ n\end{array}\right)\right) \epsilon^{2} \cdot \max \left\{1,(\operatorname{Lip} f)^{n-1}\right\}^{2}$. Logo, $\forall x \in D_{g}=D_{f}$ :

$$
(\mathrm{J} g(x))^{2} \leq(\mathrm{J} f(x))^{2}+\left(\left(\begin{array}{c}
m+n \\
n
\end{array}\right)-\left(\begin{array}{c}
m \\
n
\end{array}\right)\right) \epsilon^{2} \cdot \max \left\{1,(\operatorname{Lip} f)^{n-1}\right\}^{2} .
$$

Em particular, se $x \in A \subset J_{f}^{0}$, segue $\mathrm{J} g(x) \leq C \epsilon$, onde

$$
C:=\sqrt{\left(\begin{array}{c}
m+n \\
n
\end{array}\right)-\left(\begin{array}{c}
m \\
n
\end{array}\right)} \max \left\{1,(\operatorname{Lip} f)^{n-1}\right\},
$$

o que conclui a prova da afirmação.

3) Caso geral: $A \subset D_{f}$. É uma consequência direta dos casos 1 e 2 :

$$
\begin{aligned}
\int_{A} \mathrm{~J} f \mathrm{~d} \mathcal{L}^{n} & =\int_{A \cap J_{f}^{+}} \mathrm{J} f \mathrm{~d} \mathcal{L}^{n}+\int_{A \cap J_{f}^{0}} \mathrm{~J} f \mathrm{~d} \mathcal{L}^{n}= \\
& =\int_{\mathbb{R}^{m}} \mathcal{H}^{0}\left(A \cap J_{f}^{+} \cap f^{-1}\{y\}\right) \mathrm{d} \mathcal{H}^{n}(y)+\int_{\mathbb{R}^{m}} \mathcal{H}^{0}\left(A \cap J_{f}^{0} \cap f^{-1}\{y\}\right) \mathrm{d} \mathcal{H}^{n}(y)= \\
& =\int_{\mathbb{R}^{m}} \mathcal{H}^{0}\left(A \cap f^{-1}\{y\}\right) \mathrm{d} \mathcal{H}^{n}(y) .
\end{aligned}
$$

Corolário 3.10 (Teorema de Mudança de Variáveis). Seja $f: \mathbb{R}^{n} \rightarrow \mathbb{R}^{m}$ Lipschitz, com $n \leq m$. Então, para toda $g: \mathbb{R}^{n} \rightarrow \mathbb{R} \mathcal{L}^{n}$-mensurável com $g \geq 0$ ou g somável,

$$
\int_{\mathbb{R}^{n}} g \mathrm{~J} f \mathrm{~d} \mathcal{L}^{n}=\int_{\mathbb{R}^{m}}\left(\sum_{x \in f^{-1}\{y\}} g(x)\right) \mathrm{d} \mathcal{H}^{n}(y) .
$$

Demonstração. Suponha $g \geq 0$. Pela proposição 2.33 , existe uma sequência $\left(A_{i}\right)_{i \in \mathbb{N}}$ em $\sigma\left(\mathcal{L}^{n}\right)$ tal 
que

$$
g=\sum_{i=1}^{\infty} \frac{1}{i} \chi_{A_{i}} .
$$

Seja $\psi: \mathbb{R}^{m} \rightarrow[0, \infty]$ dada por $\psi(y):=\sum_{x \in f^{-1}\{y\}} g(x)$. Dado $y \in \mathbb{R}^{m}$, podemos calcular $\sum_{x \in f^{-1}\{y\}} g(x)$ através do teorema da convergência monótona (integrando com respeito à medida de contagem em $\left.f^{-1}\{y\}\right)$ :

$$
\begin{aligned}
\psi(y)=\sum_{x \in f^{-1}\{y\}} g(x) & =\sum_{x \in f^{-1}} \sum_{\{y\}} \frac{1}{i} \chi_{i \in \mathbb{N}}(x) \stackrel{\mathrm{TCM}}{=} \\
& =\sum_{i \in \mathbb{N}} \frac{1}{i} \sum_{x \in f^{-1}\{y\}} \chi_{A_{i}}(x)= \\
& =\sum_{i \in \mathbb{N}} \frac{1}{i} \mathcal{H}^{0}\left(A_{i} \cap f^{-1}\{y\}\right) .
\end{aligned}
$$

Como, para todo $i \in \mathbb{N}$, a função de multiplicidade $N\left(\left.f\right|_{A_{i}}\right): y \mapsto \mathcal{H}^{0}\left(A_{i} \cap f^{-1}\{y\}\right)$ é $\mathcal{H}^{n}$-mensurável, pelo lema 3.7.ii), segue que $\psi$ é $\mathcal{H}^{n}$-mensurável e $\geq 0$. Além disso, aplicando uma vez mais o teorema da convergência monótona e a fórmula da área, obtém-se:

$$
\begin{aligned}
\int_{\mathbb{R}^{m}} \psi(y) \mathrm{d} \mathcal{H}^{n}(y) & =\int_{\mathbb{R}^{m}} \sum_{i \in \mathbb{N}} \frac{1}{i} \mathcal{H}^{0}\left(A_{i} \cap f^{-1}\{y\}\right) \mathrm{d} \mathcal{H}^{n}(y) \stackrel{\mathrm{TCM}}{=} \\
& =\sum_{i \in \mathbb{N}} \frac{1}{i} \int_{\mathbb{R}^{m}} \mathcal{H}^{0}\left(A_{i} \cap f^{-1}\{y\}\right) \mathrm{d} \mathcal{H}^{n}(y) \stackrel{\mathrm{AF} 3.9}{=} \\
& =\sum_{i \in \mathbb{N}} \frac{1}{i} \int_{A_{i}} \mathrm{~J} f \mathrm{~d} \mathcal{L}^{n} \stackrel{\mathrm{T}}{=} \\
& =\int_{\mathbb{R}^{n}} \sum_{i \in \mathbb{N}} \frac{1}{i} \chi_{A_{i}} \mathrm{~J} f \mathrm{~d} \mathcal{L}^{n}=\int_{\mathbb{R}^{n}} g \mathrm{~J} f \mathrm{~d} \mathcal{L}^{n},
\end{aligned}
$$

o que prova o caso $g \geq 0$.

Se $g: \mathbb{R}^{n} \rightarrow \mathbb{R}$ é $\mathcal{L}^{n}$-somável, escrevemos $g=g^{+}-g^{-}$e aplicamos o caso já provado a $g^{+}$e $g^{-}$, o que conclui a demonstração.

Corolário 3.11. Sejam $f: \mathbb{R}^{n} \rightarrow \mathbb{R}^{m}$ be Lipschitz 1-1 e $n \leq m$. Se $g: \mathbb{R}^{n} \rightarrow \mathbb{R}$ é $\mathcal{L}^{n}$-mensurável com $g \geq 0$ or $g \in \mathrm{L}^{1}\left(\mathcal{L}^{n}\right)$, então $\int_{\operatorname{Im} f} g \circ f^{-1} \mathrm{~d} \mathcal{H}^{n}=\int_{\mathbb{R}^{n}} g \mathrm{~J} f \mathrm{~d} \mathcal{L}^{n}$. Em particular, se $g: \operatorname{Im} f \rightarrow$ $[0, \infty]$ for boreliana, então

$$
\int_{\operatorname{Im} f} g \mathrm{~d} \mathcal{H}^{n}=\int g \circ f \mathrm{~J} f \mathrm{~d} \mathcal{L}^{n}
$$

Demonstração. $\forall y \in \mathbb{R}^{m}$,

$$
\sum_{x \in f^{-1}\{y\}} g(x)= \begin{cases}g \circ f^{-1}(y) & y \in \operatorname{Im} f \\ 0 & y \in \mathbb{R}^{m} \backslash \operatorname{Im} f .\end{cases}
$$

Portanto, segue do corolário 3.10 que $\int_{\operatorname{Im} f} g \circ f^{-1} \mathrm{~d} \mathcal{H}^{n}=\int_{\mathbb{R}^{n}} g \mathrm{~J} f \mathrm{~d} \mathcal{L}^{n}$. Se $g: \operatorname{Im} f \rightarrow[0, \infty]$ for boreliana, podemos aplicar a última igualdade para $g \circ f$ no lugar de $g$, obtendo-se (3.9).

A fórmula da área e seus corolários continuam válidos se $f: \mathbb{R}^{n} \rightarrow \mathbb{R}^{m}$ for substituída por $f: \Omega \subset \mathbb{R}^{n} \rightarrow \mathbb{R}^{m}$ localmente Lipschitz, com $\Omega \subset \mathbb{R}^{n}$ aberto. Ou seja, vale o seguinte teorema:

Teorema 3.12 (fórmula da área para aplicações localmente Lipschitz). Sejam $n \leq m, \Omega \subset \mathbb{R}^{n}$ aberto e $f: \Omega \rightarrow \mathbb{R}^{m}$ localmente Lipschitz. 
i) (fórmula da área) Para todo $\mathcal{L}^{n}$-mensurável $A \subset \Omega$, a função de multiplicidade $N\left(\left.f\right|_{A}\right): \mathbb{R}^{m} \rightarrow$ $[0, \infty], y \mapsto \mathcal{H}^{0}\left(A \cap f^{-1}\{y\}\right)$, é $\mathcal{H}^{n}$-mensurável e

$$
\int_{A} \mathrm{~J} f \mathrm{~d} \mathcal{L}^{n}=\int_{\mathbb{R}^{m}} \mathcal{H}^{0}\left(A \cap f^{-1}\{y\}\right) \mathrm{d} \mathcal{H}^{n}(y) .
$$

ii) (teorema de mudança de variáveis) Se $g: \Omega \rightarrow \mathbb{R}$ é $\left.\mathcal{L}^{n}\right|_{\Omega}$-mensurável e $g \geq 0$ ou $g \in \mathrm{L}^{1}\left(\left.\mathcal{L}^{n}\right|_{\Omega}\right)$, então

$$
\int_{\Omega} g \mathrm{~J} f \mathrm{~d} \mathcal{L}^{n}=\int_{\mathbb{R}^{m}}\left(\sum_{x \in f^{-1}\{y\}} g(x)\right) \mathrm{d} \mathcal{H}^{n}(y),
$$

com o significado de que a integral no segundo membro faz sentido e vale a igualdade. Em particular, se f for 1-1, segue que $\int_{\Omega} g \mathrm{~J} f \mathrm{~d} \mathcal{L}^{n}=\int_{\operatorname{Im} f} g \circ f^{-1} \mathrm{~d} \mathcal{H}^{n}$.

A prova se obtém por um argumento direto, combinando-se a versão já provada para aplicações Lipschitz 3.9 com o teorema de extensão de Kirszbraun 2.95 e com o teorema da convergência monótona.

Como aplicações $C^{1}$ em abertos de $\mathbb{R}^{n}$ são localmente Lipschitz, seguem como corolários do teorema acima as versões do teorema de mudança de variáveis geralmente enunciadas em textos clássicos de Cálculo Avançado ou Medida e Integração, a exemplo de [6, 2.47] e [15, apêndice H].

Observação 3.13. Mais geralmente, a fórmula da área permanece válida para aplicações $f: A \subset$ $\mathbb{R}^{n} \rightarrow \mathbb{R}^{m}$ aproximadamente diferenciáveis no sentido de [9,3.1.2], onde $A \subset \mathbb{R}^{n}$ Lebesguemensurável. A prova é consequência direta da versão aqui provada para aplicações Lipschitz e do teorema de estrutura [9, 3.1.8], o qual garante a existência de uma partição do domínio $A$ de $f$ numa união enumerável de conjuntos Lebesgue-mensuráveis, restrita a cada um dos quais $f$ é Lipschitz.

\subsubsection{Aplicações da Fórmula da Área}

Eis alguns exemplos de aplicação da fórmula da área:

\section{Comprimento de uma Curva}

Sejam $-\infty<a<b<\infty$ e $\gamma:[a, b] \rightarrow \mathbb{R}^{m}$ Lipschitz 1-1. Podemos estender $\gamma$ a uma aplicação Lipschitz em $\mathbb{R}$, a qual ainda denotaremos por $\gamma$. Note que, para todo $t$ no conjunto $D_{\gamma} \in \mathscr{B}_{\mathbb{R}}$ dos pontos de diferenciabilidade de $\gamma$,

$$
\mathrm{J} \gamma(t)=\left\|\gamma^{\prime}(t)\right\|
$$

Portanto, segue do teorema de mudança de variáveis $3.10 \operatorname{com} g=\chi_{[a, b]}$ que

$$
\begin{aligned}
\int_{a}^{b}\left\|\gamma^{\prime}(t)\right\| \mathrm{d} t & =\int_{\mathbb{R}} \chi_{[a, b]} \mathrm{J} \gamma \mathrm{d} \mathcal{L}^{1} \stackrel{3.10}{=} \\
& =\int_{\mathbb{R}^{m}}\left(\sum_{x \in \gamma^{-1}\{y\}} \chi_{[a, b]}(x)\right) \mathrm{d} \mathcal{H}^{1}(y)= \\
& =\int_{\mathbb{R}^{m}} \chi_{\gamma([a, b])}(y) \mathrm{d} \mathcal{H}^{1}(y)= \\
& =\mathcal{H}^{1}(\gamma([a, b])) .
\end{aligned}
$$

\section{Área de um Gráfico}

Sejam $g: \mathbb{R}^{n} \rightarrow \mathbb{R}$ Lipschitz e $f: \mathbb{R}^{n} \rightarrow \mathbb{R}^{n+1}$ dada por $f(x):=(x, g(x))$. Então $f$ é Lipschitz 1-1 e, calculando através da fórmula de Binet-Cauchy 2.104, $\forall x \in D_{f}=D_{g}$,

$$
J f(x)=\sqrt{1+\|\nabla g(x)\|^{2}} .
$$


Para cada $U \subset \mathbb{R}^{n}$ aberto, podemos aplicar o corolário 3.11 para se obter uma fórmula clássica para a "área superficial" do gráfico de $g$ restrita a $U$, i.e. a medida de Hausdorff $n$-dimensional de $\Gamma=\Gamma(g ; U):=\{(x, g(x)) \mid x \in U\}=f(U)$ :

$$
\mathcal{H}^{n}(\Gamma)=\int_{U} \mathrm{~J} f \mathrm{~d} \mathcal{L}^{n}=\int_{U} \sqrt{1+\|\nabla g(x)\|^{2}} \mathrm{~d} x .
$$

\section{Medidas de Lebesgue e de Hausdorff numa Variedade Riemanniana}

Seja M uma variedade diferenciável $n$-dimensional com base enumerável de abertos e de classe $\mathrm{C}^{\mathrm{k}}, k \geq 1$, e $\mathrm{g}$ um tensor métrico de classe $\mathrm{C}^{\mathrm{k}-1}$ em $\mathrm{M}$. Construiremos uma medida de Radon positiva em $\mathrm{M}$ induzida pelo tensor métrico. Para tal, definimos um funcional linear positivo $I$ em $\mathrm{C}_{\mathrm{c}}(\mathrm{M})$ da seguinte forma:

1) Seja $\varphi=\left(x^{1}, \ldots, x^{n}\right): \mathcal{U} \rightarrow \mathbb{R}^{n}$ uma carta $C^{k}$ definida num aberto $\mathcal{U} \subset \mathrm{M}$. Para cada $f \in \mathrm{C}_{\mathrm{c}}(\mathrm{M})$ com spt $f \subset U$, definimos

$$
I_{\varphi}(f):=\int_{\varphi(\mathcal{U})} f \circ \varphi^{-1} \sqrt{\mathrm{g}_{\varphi}} \mathrm{d} \mathcal{L}^{n}
$$

onde $\mathrm{g}_{\varphi}: \varphi(\mathcal{U}) \rightarrow \mathbb{R}$ é definida por, para todo $x \in \mathcal{U}$,

$$
\mathrm{g}_{\varphi}(\varphi(x))=\operatorname{det} \mathrm{g}\left(\left.\frac{\partial}{\partial x_{i}}\right|_{x},\left.\frac{\partial}{\partial x_{j}}\right|_{x}\right)
$$

Se $\psi$ for outra carta $C^{k}$ em $M$ e spt $f \subset \operatorname{dom} \varphi \cap \operatorname{dom} \psi$, decorre do teorema de mudança de variáveis clássico (o qual é corolário da fórmula da área, como vimos anteriormente) que $I \varphi(f)=I_{\psi}(f)$.

2) Dada $f \in \mathrm{C}_{\mathrm{c}}(\mathrm{M})$, podemos cobrir o suporte de $f$ (que é um compacto) por um conjunto finito de vizinhanças coordenadas; digamos, spt $f \subset \cup_{i=1}^{m} \mathcal{U}_{i}$, com $\varphi_{i}: \mathcal{U}_{i} \rightarrow \mathbb{R}^{n}$ carta $C^{\mathrm{k}}$ para $1 \leq i \leq m$. Para $1 \leq i \leq m$, seja $\xi_{i} \in \mathrm{C}_{\mathrm{c}}^{\mathrm{k}}(\mathrm{M})$ com spt $\xi_{i} \subset \mathcal{U}_{i}, 0 \leq \xi_{i} \leq 1$ e tal que $\sum_{i=1}^{m} \xi_{i} \equiv 1$ numa vizinhança de spt $f$. Defina

$$
I(f):=\sum_{i=1}^{m} I_{\varphi_{i}}\left(\xi_{i} f\right) .
$$

Uma verificação direta e clássica mostra que $I$ está bem definido (i.e. a definição independe da cobertura $\left(\mathcal{U}_{i}\right)_{i}$ e da partição da unidade tomadas) e é um funcional linear positivo em $\mathrm{C}_{\mathrm{c}}(\mathrm{M})$. Portanto, pelo teorema de representação de Riesz para funcionais lineares positivos 2.83, existe uma única medida de Radon $\mu_{\mathrm{g}}$ em $\mathrm{M}$ que representa o referido funcional.

Definição 3.14 (medida de Lebesgue de uma variedade riemanniana). Com a notação acima, a medida de Radon $\mu_{\mathrm{g}}$ chama-se medida de Lebesgue da variedade riemanniana $(\mathrm{M}, \mathrm{g})$.

Suponha, agora, que $(\mathrm{M}, \mathrm{g})$ seja subvariedade riemanniana de $\mathbb{R}^{m}$ para algum $m \geq n$. Podemos fazer tal suposição sem perda de generalidade se $\mathrm{M}$ for de classe $\mathrm{C}^{\mathrm{k}}$ com $k \geq 3$, tomando-se um mergulho isométrico de Nash em algum $\mathbb{R}^{m}$ com $m$ suficientemente grande. Mostraremos, como corolário da fórmula da área, que a medida de Lebesgue $\mu_{\mathrm{g}}$ da variedade riemanniana $(\mathrm{M}, \mathrm{g})$ coincide com o traço da medida de Hausdorff $n$-dimensional de $\mathbb{R}^{m}$ em $\mathrm{M}$; ou, o que é a mesma coisa, em vista da proposição 2.49.i), com a medida de Hausdorff $n$-dimensional de M como subespaço métrico de $\mathbb{R}^{m}$. Isso generaliza o teorema 2.54 .

Teorema 3.15 (a medida de Lebesgue em $\left(\mathrm{M}^{n}, \mathrm{~g}\right)$ coincide com $\left.\left.\mathcal{H}^{n}\right|_{\mathrm{M}}\right)$. Sejam $(\mathrm{M}, \mathrm{g})$ subvariedade riemmanniana de $\mathbb{R}^{m}$, com $n \leq m$ e $\mathrm{M}$ de classe $\mathrm{C}^{\mathrm{k}}$ com $k \geq 1$. Então a medida de Lebesgue $\mu_{\mathrm{g}}$ induzida pelo tensor métrico coincide com o traço $\left.\mathcal{H}^{n}\right|_{\mathrm{M}}$, onde $\mathcal{H}^{n}$ é a medida de Hausdorff $n$-dimensional em $\mathbb{R}^{m}$. 
Demonstração. Verificaremos que $\left.\mathcal{H}^{n}\right|_{\mathrm{M}}$ é uma medida de Radon e representa o funcional linear positivo $I: \mathrm{C}_{\mathrm{c}}(\mathrm{M}) \rightarrow \mathbb{R}$ definido em (3.12) e (3.10). A tese seguirá, então, da unicidade estabelecida pelo teorema de representação de Riesz 2.83 .

1) Pela proposição 2.49.i), $\left.\mathcal{H}^{n}\right|_{M}$ coincide com a medida de Hausdorff $n$-dimensional de $\mathrm{M}$ como subespaço métrico de $\mathbb{R}^{m}$; como esta última é uma medida Borel-regular, em vista de 2.49.v), conclui-se que $\left.\mathcal{H}^{n}\right|_{\mathrm{M}}$ é Borel-regular.

2) Seja $\varphi: \mathcal{U} \rightarrow \mathbb{R}^{n}$ uma carta em $M$. Então $\varphi^{-1}: \varphi(\mathcal{U}) \rightarrow \mathbb{R}^{m}$ é uma aplicação 1-1 de classe $C^{k}$, $k \geq 1$, no aberto $\varphi(\mathcal{U}) \subset \mathbb{R}^{n}$. Em particular, é localmente Lipschitz, de modo que a fórmula da área 3.12 se aplica. Além disso, por um cálculo direto,

$$
\mathrm{J} \varphi^{-1}=\sqrt{\mathrm{g}_{\varphi}}: \varphi(\mathcal{U}) \rightarrow \mathbb{R}
$$

onde $g_{\varphi}$ é dada por (3.11).

3) De 3.12.i) segue que, para todo $K \subset \varphi(\mathcal{U})$ compacto,

$$
\mathcal{H}^{n}(\varphi(K))=\int_{K} \sqrt{\mathrm{g}_{\varphi}} \mathrm{d} \mathcal{L}^{n}<\infty .
$$

Daí, pela arbitrariedade da carta $\varphi$ tomada, conclui-se que $\left.\mathcal{H}^{n}\right|_{\mathrm{M}}$ é localmente finita. Sendo Borel-regular e localmente finita num espaço métrico separável localmente compacto, a referida medida é de Radon, em vista da observação 2.24.ii).

4) De 3.12.ii) segue que, para toda $f \in \mathrm{C}_{\mathrm{c}}(\mathrm{M})$ com spt $f \subset U$,

$$
\int f \mathrm{~d} \mathcal{H}^{n}=\int_{\varphi(\mathcal{U})} f \circ \varphi^{-1} \sqrt{\mathrm{g}_{\varphi}} \mathrm{d} \mathcal{L}^{n}=I_{\varphi}(f)=I(f) .
$$

Como $\left\{f \in \mathrm{C}_{\mathrm{c}}(\mathrm{M}) \mid \exists \varphi: \mathcal{U} \rightarrow \mathbb{R}^{n}\right.$ carta em $\mathrm{M}$ e spt $\left.f \subset \mathcal{U}\right\}$ é um conjunto de geradores para o espaço vetorial $\mathrm{C}_{\mathrm{c}}(\mathrm{M})$, conclui-se que a integral contra a medida de Radon $\left.\mathcal{H}^{n}\right|_{\mathrm{M}}$ representa $I$. Por unicidade da representação, conclui-se que $\left.\mathcal{H}^{n}\right|_{\mathrm{M}}=\mu_{\mathrm{g}}$, como queríamos.

Observação 3.16. Com a notação do teorema acima, é natural indagar se a medida $\left.\mathcal{H}^{n}\right|_{\mathrm{M}}$, que coincide com a medida de Hausdorff $n$-dimensional de $M$ como subespaço métrico de $\mathbb{R}^{m}$, também coincide com a medida de Hausdorff $n$-dimensional induzida pela distância riemanniana de $\mathrm{M}$, i.e. a distância intrínseca induzida pelo tensor métrico tomando-se ínfimos de comprimentos de arcos seccionalmente $\mathrm{C}^{1}$ ligando dois pontos. Noutras palavras, a questão que se coloca é: a distância euclidiana do ambiente $\mathbb{R}^{m}$ restrita a $\mathrm{M}$ e a distância intrínseca de $(\mathrm{M}, \mathrm{g})$ induzem a mesma medida de Hausdorff $n$-dimensional? A resposta é afirmativa e decorre do teorema IV.1.7 em [10].

\subsection{Fórmula da Coárea em Espaços Euclidianos}

Nesta seção, assumiremos $n \geq m$, salvo menção explícita em contrário.

Lema 3.17 (fórmula da coárea, caso linear). Sejam $L: \mathbb{R}^{n} \rightarrow \mathbb{R}^{m}$ linear, $n \geq m$ e $A \in \sigma\left(\mathcal{L}^{n}\right)$. Então:

i) $N\left(\left.L\right|_{A}\right): \mathbb{R}^{m} \rightarrow[0, \infty]$ dada por $N\left(\left.L\right|_{A}\right)(y):=\mathcal{H}^{n-m}\left(A \cap L^{-1}\{y\}\right)$ is $\mathcal{L}^{m}$-mensurável.

ii)

$$
\int_{\mathbb{R}^{m}} \mathcal{H}^{n-m}\left(A \cap L^{-1}\{y\}\right) \mathrm{d} \mathcal{L}^{m}(y)=\llbracket L \rrbracket \mathcal{L}^{n}(A)
$$


Demonstração. Sejam $O \in \mathrm{O}(m, n)$ e $S \in \operatorname{Sym}(m)$ dados pelo teorema 2.101, i.e. tais que $L=S \circ O^{*}$ é uma decomposição polar de $L$.

1. Caso 1: $\operatorname{dim} \operatorname{Im} L<m$. Então, para $\mathcal{L}^{m}$-q.t. $y \in \mathbb{R}^{m}, L^{-1}\{y\}=\emptyset$, $\operatorname{logo} N\left(\left.L\right|_{A}\right)(y)=\mathcal{H}^{n-m}(A \cap$ $\left.L^{-1}\{y\}\right)=0$. Ou seja, $N\left(\left.L\right|_{A}\right)$ é nulo $\mathcal{L}^{m}$-q.s., portanto é $\mathcal{L}^{m}$-mensurável. Por outro lado, como $\operatorname{Im} L=\operatorname{Im} S$, tem-se $\llbracket L \rrbracket=|\operatorname{det} S|=0$. Assim, ambos os membros de (3.13) são nulos.

2. Caso 2: $L=P: \mathbb{R}^{n} \equiv \mathbb{R}^{m} \times \mathbb{R}^{n-m} \rightarrow \mathbb{R}^{m}$ é a projeção no primeiro fator (logo $O=P^{*}$ e $S=\operatorname{id}_{\mathbb{R}^{m}}$ ). Fixe $y \in \mathbb{R}^{m}$ e seja $\mathrm{pr}_{2}: \mathbb{R}^{n} \equiv \mathbb{R}^{m} \times \mathbb{R}^{n-m} \rightarrow \mathbb{R}^{n-m}$ a projeção no segundo fator. Então a restrição $\operatorname{pr}_{2}: P^{-1}\{y\} \rightarrow \mathbb{R}^{n-m}$ é uma isometria que leva $A \cap P^{-1}\{y\}$ na seção $A_{y}=\{x \mid(y, x) \in A\} \subset \mathbb{R}^{n-m}$. Portanto, da proposição 2.49 partes i) e ii) e do teorema 2.54, conclui-se que $N\left(\left.P\right|_{A}\right)(y)=\mathcal{H}^{n-m}\left(A \cap P^{-1}\{y\}\right)=\mathcal{H}^{n-m}\left(A_{y}\right)=\mathcal{L}^{n-m}\left(A_{y}\right)$. Logo, do teorema de Fubini-Tonelli 2.39 aplicado à medida produto $\mathcal{L}^{m} \times \mathcal{L}^{m-n}$ (a qual coincide com $\mathcal{L}^{n}$, em vista do exemplo 2.41), conclui-se que $N\left(\left.P\right|_{A}\right)$ é $\mathcal{L}^{m}$-mensurável e $\int_{\mathbb{R}^{m}} N\left(\left.P\right|_{A}\right) \mathrm{d} \mathcal{L}^{m}=\mathcal{L}^{n}(A)$, o que prova (3.13) (pois $\llbracket P \rrbracket=1$ ).

3. Caso 3: $L: \mathbb{R}^{n} \rightarrow \mathbb{R}^{m}$ sobrejetiva. Note que, como $\operatorname{Im} L=\operatorname{Im} S$, tem-se $S \in \operatorname{Sym}(m) \cap G L\left(\mathbb{R}^{m}\right)$. Afirmamos que existe $Q \in \mathrm{O}(n)$ tal que $O^{*}=P \circ Q$, onde $P: \mathbb{R}^{n} \equiv \mathbb{R}^{m} \times \mathbb{R}^{n-m} \rightarrow \mathbb{R}^{m}$ é a projeção no primeiro fator, como no item anterior. De fato, estenda $O \in \mathrm{O}(m, n)$ a uma isometria linear $S: \mathbb{R}^{n} \equiv \mathbb{R}^{m} \times \mathbb{R}^{n-m} \rightarrow \mathbb{R}^{n}$ e defina $Q:=S^{*}$. Como $P^{*}: \mathbb{R}^{m} \rightarrow \mathbb{R}^{m} \times \mathbb{R}^{n-m}$ é a inclusão no primeiro fator, tem-se $S \circ P^{*}=O, \log \circ O^{*}=P \circ S^{*}=P \circ Q$, como queríamos. Com $Q \in \mathrm{O}(n)$ dada pela afirmação acima, tem-se, $\forall y \in \mathbb{R}^{m}$,

$$
\begin{aligned}
N\left(\left.L\right|_{A}\right)(y) & =\mathcal{H}^{n-m}\left(A \cap L^{-1}\{y\}\right)= \\
& =\mathcal{H}^{n-m}\left(A \cap(S \circ P \circ Q)^{-1}\{y\}\right)= \\
& =\mathcal{H}^{n-m}\left(Q^{-1}\left[Q(A) \cap P^{-1}\left\{S^{-1}(y)\right\}\right]\right) \stackrel{2.49 . i i)}{=} \\
& =\mathcal{H}^{n-m}\left(Q(A) \cap P^{-1}\left\{S^{-1}(y)\right\}\right)= \\
& =N\left(\left.P\right|_{Q(A)}\right) \circ S^{-1}(y) .
\end{aligned}
$$

Ou seja, $N\left(\left.L\right|_{A}\right)=N\left(\left.P\right|_{Q(A)}\right) \circ S^{-1}$. Pelo item anterior, $N\left(\left.P\right|_{Q(A)}\right)$ é $\mathcal{L}^{m}$-mensurável, e $S^{-1}$ é contínua, portanto boreliana; então segue que a composta $N\left(\left.L\right|_{A}\right)=N\left(\left.P\right|_{Q(A)}\right) \circ S^{-1}$ é $\mathcal{L}^{m}$-mensurável e $\geq 0$. Além disso,

$$
\begin{aligned}
\int_{\mathbb{R}^{m}} N(f \mid A)(y) \mathrm{d} \mathcal{L}^{m}(y) & =\int_{\mathbb{R}^{m}} N\left(\left.P\right|_{Q(A)}\right) \circ S^{-1}(y) \mathrm{d} \mathcal{L}^{m}(y) \stackrel{3.5 . i i)}{=} \\
& =|\operatorname{det} S| \int_{\mathbb{R}^{m}} N\left(\left.P\right|_{Q(A)}\right) \mathrm{d} \mathcal{L}^{m} \stackrel{\text { Caso }}{=} 2 \\
& =|\operatorname{det} S| \mathcal{L}^{n}(Q(A)) \stackrel{Q \in \underline{\mathrm{O}}(n)}{=} \llbracket L \rrbracket \mathcal{L}^{n}(A),
\end{aligned}
$$

o que prova (3.13).

Para o próximo lema, recorde a noção de integral superior introduzida na definição 2.36. Este é o ponto mais delicado na prova da fórmula da coárea 3.23. Trata-se de um caso particular do teorema 2.10.25 em [9]; Federer o enunciou para aplicações Lipschitz $f: X \rightarrow Y$ entre espaços métricos $X$ e $Y$ com algumas hipóteses restritivas que posteriormente foram removidas - vide [2] e referências lá citadas.

Lema 3.18 (desigualdade de Eilenberg ou desigualdade da coárea). Sejam $n, m \in \mathbb{N}$ e $f: \mathbb{R}^{n} \rightarrow \mathbb{R}^{m}$ Lipschitz. Então, $\forall k, l \in[0, \infty) e \forall A \subset \mathbb{R}^{n}$,

$$
\int_{\mathbb{R}^{m}}^{*} \mathcal{H}^{k}\left(A \cap f^{-1}\{y\}\right) \mathrm{d} \mathcal{H}^{l}(y) \leq \frac{\alpha(k) \alpha(l)}{\alpha(k+l)}(\operatorname{Lip} f)^{l} \mathcal{H}^{k+l}(A) .
$$


Note que não se assume $n \geq m$ nem a mensurabilidade de $A$ no enunciado do lema acima. Provaremos apenas o caso $l=m$, para o qual é possível fazer um argumento mais simples que o de Federer, o qual adaptamos de [12] e depende fortemente da desigualdade isodiamétrica 2.53. Apenas este caso será usado na prova da fórmula da coárea.

Prova do caso $l=m$. Para cada $j \in \mathbb{N}$, pela proposição 2.49.iv) existe $\left(B_{i}^{j}\right)_{i \in \mathbb{N}}$ cobertura de $A$ por fechados com diâmetros $\leq 1 / j$ tal que

$$
\sum_{i \in \mathbb{N}} \alpha(k+m)\left(\frac{\operatorname{diam} B_{i}^{j}}{2}\right)^{k+m} \leq \mathcal{H}_{1 / j}^{k+m}(A)+\frac{1}{j} .
$$

$\forall i, j \in \mathbb{N}$, defina

$$
g_{i}^{j}:=\alpha(k)\left(\frac{\operatorname{diam} B_{i}^{j}}{2}\right)^{k} \chi_{f\left(B_{i}^{j}\right)} .
$$

Como $f\left(B_{i}^{j}\right)$ é $\sigma$-compacto (pois $B_{i}^{j}$ é fechado, logo $\sigma$-compacto, e $f$ é contínua), portanto Borelmensurável, $g_{i}^{j}$ é boreliana e $\geq 0$, e assim o é $\sum_{i \in \mathbb{N}} g_{i}^{j}: \mathbb{R}^{m} \rightarrow[0, \infty]$. Além disso, para cada $y \in \mathbb{R}^{m}$ e $j \in \mathbb{N}, A \cap f^{-1}\{y\}$ está contido na união das bolas de $\left(B_{i}^{j}\right)_{i \in \mathbb{N}}$ que intersectam $f^{-1}\{y\}$, i.e. tais que $y \in f\left(B_{i}^{j}\right)$. Daí segue que, $\forall y \in \mathbb{R}^{m}$,

$$
\mathcal{H}_{1 / j}^{k}\left(A \cap f^{-1}\{y\}\right) \leq \sum_{i \in \mathbb{N}} g_{i}^{j}(y) .
$$

Assim,

$$
\begin{aligned}
\int_{\mathbb{R}^{m}}^{*} & \mathcal{H}^{k}\left(A \cap f^{-1}\{y\}\right) \mathrm{d} \mathcal{L}^{m}(y)= \\
= & \int_{\mathbb{R}^{m}}^{*} \lim _{j \rightarrow \infty} \mathcal{H}_{1 / j}^{k}\left(A \cap f^{-1}\{y\}\right) \mathrm{d} \mathcal{L}^{m}(y) \stackrel{\text { monotonicidade de } \int^{*}}{\leq} \\
\leq & \int_{\mathbb{R}^{m}}^{*} \liminf _{j \rightarrow \infty} \sum_{i \in \mathbb{N}} g_{i}^{j}(y) \mathrm{d} \mathcal{L}^{m}(y) \stackrel{2.37 . i i)}{=} \\
= & \int_{\mathbb{R}^{m}} \liminf _{j \rightarrow \infty} \sum_{i \in \mathbb{N}} g_{i}^{j}(y) \mathrm{d} \mathcal{L}^{m}(y) \stackrel{\text { lema de Fatou }}{\leq} \\
\leq & \liminf _{j \rightarrow \infty} \int_{\mathbb{R}^{m}} \sum_{i \in \mathbb{N}} g_{i}^{j}(y) \mathrm{d} \mathcal{L}^{m}(y) \stackrel{\text { TCM }}{=} \\
= & \liminf _{j \rightarrow \infty} \sum_{i \in \mathbb{N}} \int_{\mathbb{R}^{m}} g_{i}^{j}(y) \mathrm{d} \mathcal{L}^{m}(y)= \\
= & \liminf _{j \rightarrow \infty} \sum_{i \in \mathbb{N}} \alpha(k)\left(\frac{\operatorname{diam} B_{i}^{j}}{2}\right)^{k} \mathcal{L}^{m}\left(f\left(B_{i}^{j}\right)\right) \stackrel{\text { des. isodiamétrica } 2.53}{\leq} \\
\leq & \liminf _{j \rightarrow \infty} \sum_{i \in \mathbb{N}} \alpha(k)\left(\frac{\operatorname{diam}^{j}}{2}\right)^{k} \alpha(m)\left(\frac{\text { diam } f\left(B_{i}^{j}\right)}{2}\right)^{m} \leq \\
\leq & \frac{\alpha(k) \alpha(m)}{\alpha(k+m)}(\operatorname{Lip} f)^{m} \liminf _{j \rightarrow \infty} \sum_{i \in \mathbb{N}} \alpha(k+m)\left(\frac{\text { diam } B_{i}^{j}}{2}\right)^{k+m}(3.14) \\
\leq & \frac{\alpha(k) \alpha(m)}{\alpha(k+m)}(\operatorname{Lip} f)^{m} \liminf _{j \rightarrow \infty}\left(\mathcal{H}_{1 / j}^{k+m}(A)+\frac{1}{j}\right)= \\
= & \frac{\alpha(k) \alpha(m)}{\alpha(k+m)}(\operatorname{Lip} f)^{m} \mathcal{H}^{k+m}(A) .
\end{aligned}
$$


Lema 3.19. Sejam $f: \mathbb{R}^{n} \rightarrow \mathbb{R}^{m}$ Lipschitz com $n \geq m, A \in \sigma\left(\mathcal{L}^{n}\right)$. Então:

i) Para $\mathcal{L}^{m}$-q.t. $y \in \mathbb{R}^{m}, A \cap f^{-1}\{y\}$ é $\mathcal{H}^{n-m}$-mensurável.

ii) $N\left(\left.f\right|_{A}\right): \mathbb{R}^{m} \rightarrow[0, \infty]$ dada por $N\left(\left.f\right|_{A}\right)(y):=\mathcal{H}^{n-m}\left(A \cap f^{-1}\{y\}\right)$ é $\mathcal{L}^{m}$-mensurável.

\section{Demonstração.}

1) Caso 1: $A$ é compacto. Fixe $t \geq 0$. Para cada $i \in \mathbb{N}$, seja $U_{i}$ o conjunto dos pontos $y \in \mathbb{R}^{m}$ tais que existe um conjunto finito de abertos $\left(S_{j}\right)_{1 \leq j \leq k}$ satisfazendo as seguintes condições:

$$
\left\{\begin{array}{l}
A \cap f^{-1}\{y\} \subset \cup_{j=1}^{k} S_{j}, \\
\operatorname{diam} S_{j} \leq \frac{1}{i}, \forall 1 \leq j \leq k, \\
\sum_{j=1}^{k} \alpha(n-m)\left(\frac{\operatorname{diam} S_{j}}{2}\right)^{n-m} \leq t+\frac{1}{i} .
\end{array}\right.
$$

2) Afirmação \#1: $\forall i \in \mathbb{N}, U_{i}$ é aberto. De fato, seja $y \in U_{i}$. Tome $\left(S_{j}\right)_{1 \leq j \leq k}$ satisfazendo as condições (3.15). Provemos a existência de $r>0$ tal que $A \cap f^{-1}(\mathbb{U}(y, r)) \subset \cup_{j=1}^{k} S_{j}$, donde se conclui que $\mathbb{U}(y, r) \subset U_{i}$, o que prova a afirmação. A existência de tal $r>0$ é consequência direta do fato de que $A$ é compacto e $f$ é contínua: se não existisse tal $r>0$, poderíamos tomar uma sequência $\left(y_{h}\right)_{h \in \mathbb{N}}$ in $\mathbb{R}^{m}$ convergente para $y$ tal que, $\forall h \in \mathbb{N}$, existe $x_{h} \in f^{-1}\left\{y_{h}\right\} \cap A \backslash \cup_{1 \leq j \leq k} S_{j}$. Como $A \backslash \cup_{1 \leq j \leq k} S_{j}$ é compacto, existiria uma subsequência de $\left(x_{h}\right)_{h \in \mathbb{N}}$, a qual ainda denotaremos por $\left(x_{h}\right)_{h \in \mathbb{N}}$, tal que $x_{h} \rightarrow x \in A \backslash \cup_{1 \leq j \leq k} S_{j}$. Por continuidade, segue que $f(x)=\lim f\left(x_{h}\right)=$ $\lim y_{h}=y, \operatorname{logo} x \in f^{-1}\{y\} \backslash \cup_{1 \leq j \leq k} S_{j}$, chegando-se a uma contradição.

3) Afirmação \#2: $\left\{y \in \mathbb{R}^{m} \mid \mathcal{H}^{n-m}\left(A \cap f^{-1}\{y\}\right) \leq t\right\}=\cap_{i \in \mathbb{N}} U_{i}$, logo é um boreliano. Como $\left\{y \in \mathbb{R}^{m} \mid \mathcal{H}^{n-m}\left(A \cap f^{-1}\{y\}\right) \leq t\right\}=\emptyset$ para $t<0$, a afirmação implica que $N\left(\left.f\right|_{A}\right)$ é boreliana se $A$ for compacto. Como, $\forall y \in \mathbb{R}^{m}, A \cap f^{-1}\{y\}$ é compacto, logo boreliano, a prova da afirmação concluirá a demonstração do caso 1 .

Prova da afirmação \#2:

- Assuma que $\mathcal{H}^{n-m}\left(A \cap f^{-1}\{y\}\right) \leq t$. Então, $\forall \delta>0, \mathcal{H}_{\delta}^{n-m}\left(A \cap f^{-1}\{y\}\right) \leq t$. Dado $i \in \mathbb{N}$, escolha $\delta \in\left(0, \frac{1}{i}\right)$. Em vista da proposição 2.49.iv), existe uma cobertura enumerável $\mathcal{G}$ de $A \cap f^{-1}\{y\}$ por abertos de $\mathbb{R}^{n}$ com diâmetros $\leq \delta$ tal que $\sum_{S \in \mathcal{G}} \alpha(n-m)\left(\frac{\operatorname{diam} S}{2}\right)^{n-m}<$ $t+\frac{1}{i}$. Como $A \cap f^{-1}\{y\}$ é compacto, podemos extrair uma subcobertura finita $\left(S_{j}\right)_{1 \leq j \leq k}$ de $\mathcal{G}$ satisfazendo (3.15), de modo que $y \in U_{i}$. Como $i \in \mathbb{N}$ é arbitrário, segue que $y \in \cap_{i \in \mathbb{N}} U_{i}$.

- Reciprocamente, se $\forall i \in \mathbb{N}, y \in U_{i}$, então (3.15) garante que $\mathcal{H}_{1 / i}^{n-m}\left(A \cap f^{-1}\{y\}\right) \leq t+\frac{1}{i}$; portanto, tomando $i \rightarrow \infty$, conclui-se que $\mathcal{H}^{n-m}\left(A \cap f^{-1}\{y\}\right) \leq t$.

4) Caso 2: $A$ é $\sigma$-compacto (em particular, isso vale se $A$ é aberto). Então, $\forall y \in \mathbb{R}^{m}, A \cap f^{-1}\{y\}$ é $\sigma$-compacto, logo boreliano. Além disso, podemos tomar uma sequência crescente $\left(K_{i}\right)_{i \in \mathbb{N}}$ de subconjuntos compactos de $A$ cuja união é $A$, de modo que, $\forall y \in \mathbb{R}^{m}$, a sequência de borelianos $\left(K_{i} \cap f^{-1}\{y\}\right)_{i \in \mathbb{N}}$ cresce para $A \cap f^{-1}\{y\}$. Daí, aplicando a continuidade para cima 2.9.i) para $\mathcal{H}^{n-m}$, segue que $N\left(\left.f\right|_{K_{i}}\right)$ cresce pontualmente para $N\left(\left.f\right|_{A}\right)$; do caso 1 e do teorema 2.31.iv) conclui-se que $N\left(\left.f\right|_{A}\right)$ é boreliana.

5) Caso 3: $\mathcal{L}^{n}(A)=0$. Segue do lema $3.18 \mathrm{com} k=n-m$ e $l=m$, e do teorema 2.54, que $\int_{\mathbb{R}^{m}}^{*} N\left(\left.f\right|_{A}\right) \mathrm{d} \mathcal{L}^{m}=0$. Logo, da proposição 2.37.i $), N\left(\left.f\right|_{A}\right)$ é $\mathcal{L}^{m}$-mensurável e $\int_{\mathbb{R}^{m}} N\left(\left.f\right|_{A}\right) \mathrm{d} \mathcal{L}^{m}=$ 0 , de modo que $N\left(\left.f\right|_{A}\right)$ é $\mathcal{L}^{m}$-q.s. nula. Ou seja, para $\mathcal{L}^{m}$-q.t. $y \in \mathbb{R}^{m}, \mathcal{H}^{n-m}\left(A \cap f^{-1}\{y\}\right)=0$, donde $A \cap f^{-1}\{y\}$ é $\mathcal{H}^{n-m}$-mensurável.

6) Caso 4: $\mathcal{L}^{n}(A)<\infty$. Como $\mathcal{L}^{n}$ é uma medida de Radon, podemos tomar uma sequência decrescente $\left(U_{k}\right)_{k \in \mathbb{N}}$ de abertos contendo $A$ tal que $\inf \left\{\mathcal{L}^{n}\left(U_{k}\right) \mid k \in \mathbb{N}\right\}=\mathcal{L}^{n}(A)$. Assim, tomando $B:=\cap_{k \in \mathbb{N}} U_{k} \in \mathscr{B}_{\mathbb{R}^{n}}$, tem-se $\mathcal{L}^{n}(B)=\mathcal{L}^{n}(A)<\infty$; como $A$ é $\mathcal{L}^{n}$-mensurável, conclui-se que 
$\mathcal{L}^{n}(B \backslash A)=0$. Em particular, segue do caso 3 que, para $\mathcal{L}^{m}$-q.t. $y \in \mathbb{R}^{m},(B \backslash A) \cap f^{-1}\{y\}$ é $\mathcal{H}^{n-m}$-nulo. Para um tal $y, A \cap f^{-1}\{y\}=\left(B \cap f^{-1}\{y\}\right) \backslash\left((B \backslash A) \cap f^{-1}\{y\}\right)$ é $\mathcal{H}^{n-m}$-mensurável e $\mathcal{H}^{n-m}\left(B \cap f^{-1}\{y\}\right)=\mathcal{H}^{n-m}\left(A \cap f^{-1}\{y\}\right)$, o que mostra que $N\left(\left.f\right|_{A}\right)=N\left(\left.f\right|_{B}\right) \mathcal{L}^{m}$-q.s., de modo que o caso 4 estará concluído uma vez que provemos que $N\left(\left.f\right|_{B}\right)$ é $\mathcal{L}^{m}$-mensurável. De fato,

- Para cada $y \in \mathbb{R}^{m}$ e $k \in \mathbb{N}, U_{k} \cap f^{-1}\{y\}$ é boreliano e a sequência $\left(U_{k} \cap f^{-1}\{y\}\right)_{k \in \mathbb{N}}$ decresce para $B \cap f^{-1}\{y\}$

- como $\mathcal{L}^{n}(A)<\infty$ e $\mathcal{L}^{n}\left(U_{k}\right) \downarrow \mathcal{L}^{n}(A)$, podemos assumir que $\mathcal{L}^{n}\left(U_{1}\right)<\infty$ (descartando os primeiros termos da sequência $\left(U_{k}\right)_{k \in \mathbb{N}}$, se necessário). Então segue do lema $3.18 \mathrm{com}$ $k=n-m$ e $l=m$ que $\int_{\mathbb{R}^{m}} N\left(\left.f\right|_{U_{1}}\right) \mathrm{d} \mathcal{L}^{m}=\int_{\mathbb{R}^{m}}^{*} N\left(\left.f\right|_{U_{1}}\right) \mathrm{d} \mathcal{L}^{m}<\infty$. Portanto, para $\mathcal{L}^{m}$ q.t. $y \in \mathbb{R}^{m}, N\left(\left.f\right|_{U_{1}}\right)(y)=\mathcal{H}^{n-m}\left(U_{1} \cap f^{-1}\{y\}\right)<\infty$; para um tal $y$, podemos aplicar a continuidade para baixo 2.9.ii) para concluir que $N\left(\left.f\right|_{U_{k}}\right)(y) \downarrow N\left(\left.f\right|_{B}\right)(y)$. Ou seja, $N\left(\left.f\right|_{U_{k}}\right)$ decresce $\mathcal{L}^{m}$-q.s. para $N\left(\left.f\right|_{B}\right)$. Então segue que $N\left(\left.f\right|_{B}\right)$ é $\mathcal{L}^{m}$-mensurável (em vista do caso 2 e do teorema 2.31.iv), como afirmado.

7) Caso geral. Pela $\sigma$-finitude de $\mathcal{L}^{n}$, podemos escrever $A=\dot{\cup}_{k \in \mathbb{N}} A_{k}$, onde $\forall k \in \mathbb{N}, A_{k} \in \sigma\left(\mathcal{L}^{n}\right)$ e $\mathcal{L}^{n}\left(A_{k}\right)<\infty$. Então $A \cap f^{-1}\{y\}=\dot{\cup}_{k \in \mathbb{N}}\left(A_{k} \cap f^{-1}\{y\}\right)$. Segue do caso 4 que, para $\mathcal{L}^{m}$-q.t. $y \in \mathbb{R}^{m}$, $\forall k \in \mathbb{N}, A_{k} \cap f^{-1}\{y\}$ é $\mathcal{H}^{n-m}$-mensurável; logo, para tais $y, A \cap f^{-1}\{y\}$ é $\mathcal{H}^{n-m}$-mensurável e $N\left(\left.f\right|_{A}\right)(y)=\sum_{k \in \mathbb{N}} N\left(\left.f\right|_{A_{k}}\right)(y)$ pela $\sigma$-aditividade de $\mathcal{H}^{n-m}$. Então $N\left(\left.f\right|_{A}\right)$ é $\mathcal{L}^{m}$-mensurável, em vista do caso 4 e do teorema 2.31.iv).

Lema 3.20 (linearização Lipschitz, parte II). Sejam $t>1, h: \mathbb{R}^{n} \rightarrow \mathbb{R}^{n}$ Lipschitz e $J_{h}^{+}=$ $\left\{x \in D_{h} \mid \mathrm{J} h(x)>0\right\}$. Então existe uma família disjunta enumerável $\left(E_{k}\right)_{k \in \mathbb{N}}$ in $\mathscr{B}_{J_{h}^{+}}$tal que $\mathcal{L}^{n}\left(J_{h}^{+} \backslash \cup_{k \in \mathbb{N}} E_{k}\right)=0$ e, $\forall k \in \mathbb{N},\left.h\right|_{E_{k}}$ é $1-1$ e existe $S_{k} \in \operatorname{Sym}(n) \cap \operatorname{GL}\left(\mathbb{R}^{n}\right)$ satisfazendo

i) $\left.\operatorname{Lip} S_{k}^{-1} \circ h\right|_{E_{k}} \leq t e \operatorname{Lip}\left(\left.h\right|_{E_{k}}\right)^{-1} \circ S_{k} \leq t$;

ii) $\forall x \in E_{k},\left\|S_{k}^{-1} \circ \mathrm{D} h(x)\right\| \leq t e\left\|\mathrm{D} h(x)^{-1} \circ S_{k}\right\| \leq t$.

Observação 3.21. Com a notação do lema precedente:

1) As condições i) e ii) são equivalentes a, respectivamente:

i') $\forall x, y \in h\left(E_{k}\right)$,

$$
t^{-1}\left\|S_{k}^{-1} \cdot(x-y)\right\| \leq\left\|\left(\left.h\right|_{E_{k}}\right)^{-1}(x)-\left(\left.h\right|_{E_{k}}\right)^{-1}(y)\right\| \leq t\left\|S_{k}^{-1} \cdot(x-y)\right\| ;
$$

ii') $\forall x \in E_{k}, \forall v \in \mathbb{R}^{n}, t^{-1}\left\|S_{k}^{-1} \cdot v\right\| \leq\left\|\mathrm{D} h(x)^{-1} \cdot v\right\| \leq t\left\|S_{k}^{-1} \cdot v\right\|$.

A prova é imediata e similar ao argumento usado na proposição 3.2.

2) A condição i) implica que $\left.h\right|_{E_{k}}$ admite inversa Lipschitz, pois $\left(\left.h\right|_{E_{k}}\right)^{-1}=\left[\left(\left.h\right|_{E_{k}}\right)^{-1} \circ S_{k}\right] \circ S_{k}^{-1}$, donde $\operatorname{Lip}\left(\left.h\right|_{E_{k}}\right)^{-1} \leq t\left\|S_{k}^{-1}\right\|$.

3) A condição ii) implica que, $\forall x \in E_{k}$ :

$$
t^{-n}\left|\operatorname{det} S_{k}\right| \leq \mathrm{J} h(x) \leq t^{n}\left|\operatorname{det} S_{k}\right|
$$

De fato,

$$
\begin{aligned}
\mathrm{J} h(x) & =\left|\operatorname{det} S_{k}\right|\left|\operatorname{det} S_{k}^{-1}\right| \llbracket \operatorname{D} h(x) \rrbracket \stackrel{2.105 . i)}{=} \\
& =\left|\operatorname{det} S_{k}\right| \llbracket S_{k}^{-1} \circ \operatorname{Dh}(x) \rrbracket,
\end{aligned}
$$

logo (3.16) segue de ii) e da proposição 2.105.ii) $\operatorname{com} S_{k}^{-1} \circ \mathrm{D} h(x)$ no lugar de $T$. 


\section{Demonstração.}

1) Seja $\left(F_{k}\right)_{k \in \mathbb{N}}$ uma família enumerável disjunta em $\mathscr{B}_{\mathbb{R}^{n}}$ tal que $J_{h}^{+}=\dot{\cup}_{k \in \mathbb{N}} F_{k}$ e, $\forall k \in \mathbb{N},\left.h\right|_{F_{k}}$ é 1-1 com inversa Lipschitz. A existência de uma tal família segue do teorema 3.4 e da proposição 3.2 com $h$ no lugar de $f$.

2) Fixe $k \in \mathbb{N}$. Como $\left(\left.h\right|_{F_{k}}\right)^{-1}: h\left(F_{k}\right) \rightarrow \mathbb{R}^{n}$ é Lipschitz, pelo teorema 2.94 (ou pelo teorema 2.95) ela pode ser estendida a uma aplicação Lipschitz $h_{k}: \mathbb{R}^{n} \rightarrow \mathbb{R}^{n}$. Como $h\left(F_{k}\right) \subset\{x \in$ $\left.\mathbb{R}^{n} \mid h \circ h_{k}(x)=x\right\}$, segue do corolário 2.99 com $h_{k}$ no lugar de $f$ e $h$ no lugar de $g$ que $\mathrm{D} h\left(h_{k}(x)\right) \circ \mathrm{D} h_{k}(x)=\operatorname{id}_{\mathbb{R}^{n}}$ para $\mathcal{L}^{n}$-q.s. $x \in h\left(F_{k}\right)$. Portanto, definindo

$$
Y_{k}:=\left\{x \in \mathbb{R}^{n} \mid \exists \mathrm{D} h_{k}(x), \exists \mathrm{D} h\left(h_{k}(x)\right), \mathrm{D} h\left(h_{k}(x)\right) \circ \mathrm{D} h_{k}(x)=\mathrm{id}_{\mathbb{R}^{n}}\right\},
$$

tem-se $Y_{k} \in \mathscr{B}_{\mathbb{R}^{n}}$ e $h\left(F_{k}\right) \backslash Y_{k}$ é $\mathcal{L}^{n}$-nulo. Além disso, $\forall x \in Y_{k}, \mathrm{~J} h\left(h_{k}(x)\right) \cdot \mathrm{J} h_{k}(x)=1, \operatorname{logo}$ $\mathrm{J} h_{k}(x)>0$, donde $Y_{k} \subset J_{h_{k}}^{+}$.

3) Aplicando o teorema de linearização Lipschitz 3.4 para $h_{k}$, existem uma família enumerável disjunta $\left(G_{j}^{k}\right)_{j \in \mathbb{N}}$ em $\mathscr{B}_{\mathbb{R}^{n}}$ e uma sequência $\left(R_{j}^{k}\right)_{j \in \mathbb{N}}$ em $\operatorname{Sym}(n) \cap \mathrm{GL}\left(\mathbb{R}^{n}\right)$ tais que $J_{h_{k}}^{+}=\dot{\cup}_{j \in \mathbb{N}} G_{j}^{k}$ e, $\forall j \in \mathbb{N},\left(G_{j}^{k}, R_{j}^{k}\right)$ é uma t-linearização para $h_{k}$. Defina, para cada $j \in \mathbb{N}$,

$$
E_{j}^{k}:=F_{k} \cap h^{-1}\left(G_{j}^{k} \cap Y_{k}\right) \in \mathscr{B}_{\mathbb{R}^{n}} \quad \text { e } \quad S_{j}^{k}:=\left(R_{j}^{k}\right)^{-1} \in \operatorname{Sym}(n) \cap \mathrm{GL}\left(\mathbb{R}^{n}\right) .
$$

Provaremos que a família enumerável $\left(E_{j}^{k}, S_{j}^{k}\right)_{k, j \in \mathbb{N}}$ satisfaz as condições enunciadas no teorema.

4) É claro que $\left(E_{j}^{k}\right)_{k, j \in \mathbb{N}}$ é uma família disjunta em $\mathscr{B}_{J_{h}^{+}}$e, $\forall k, j \in \mathbb{N},\left.h\right|_{E_{j}^{k}}$ é 1-1 com inversa Lipschitz, logo $E_{j}^{k} \subset F^{k}$. A saber, $\left(\left.h\right|_{E_{j}^{k}}\right)^{-1}$ é a restrição de $h_{k}$ a $h\left(E_{j}^{k}\right)=h\left(F_{k}\right) \cap G_{j}^{k} \cap Y_{k}$.

5) Afirmamos que $J_{h}^{+} \backslash \cup_{k, n \in \mathbb{N}} E_{k, j}$ é $\mathcal{L}^{n}$-nulo. De fato, como $J_{h}^{+}=\dot{\cup}_{k \in \mathbb{N}} F_{k}$, é suficiente mostrar que, para todo $k \in \mathbb{N}, F_{k} \backslash \cup_{j \in \mathbb{N}} E_{j}^{k}$ é $\mathcal{L}^{n}$-nulo. Como $\left.h\right|_{F_{k}}$ é bi-Lipschitz sobre $h\left(F_{k}\right)$, a última condição é equivalente a $h\left(F_{k} \backslash \cup_{j \in \mathbb{N}} E_{j}^{k}\right)$ ser $\mathcal{L}^{n}$-nulo. Como

$$
\begin{aligned}
h\left(F_{k} \backslash \cup_{j \in \mathbb{N}} E_{j}^{k}\right) & =h\left(F_{k} \backslash\left[F_{k} \cap h^{-1}\left(Y_{k} \cap \cup_{j \in \mathbb{N}} G_{j}^{k}\right)\right]\right)= \\
& =h\left(F_{k} \backslash h^{-1}(\underbrace{Y_{k} \cap J_{h_{k}}^{+}}_{=Y_{k}})\right)= \\
& =h\left(F_{k}\right) \backslash Y_{k},
\end{aligned}
$$

a afirmação segue da parte 2).

6) $\forall k, j \in \mathbb{N},\left.h_{k}\right|_{G_{j}^{k}}$ estende $\left(\left.h\right|_{E_{j}^{k}}\right)^{-1}$ (pela parte 4$), \operatorname{logo}\left(\left.h_{k}\right|_{G_{j}^{k}}\right)^{-1}$ estende $\left.h\right|_{E_{j}^{k}}$. Portanto, $\forall k, j \in$ $\mathbb{N}$,

$$
\begin{aligned}
& \left.\operatorname{Lip}\left(S_{j}^{k}\right)^{-1} \circ h\right|_{E_{j}^{k}}=\left.\operatorname{Lip} R_{j}^{k} \circ h\right|_{E_{j}^{k}} \leq \operatorname{Lip} R_{j}^{k} \circ\left(\left.h_{k}\right|_{G_{j}^{k}}\right)^{-1} \leq t, \\
& \operatorname{Lip}\left(\left.h\right|_{E_{j}^{k}}\right)^{-1} \circ S_{j}^{k}=\operatorname{Lip}\left(\left.h\right|_{E_{j}^{k}}\right)^{-1} \circ\left(R_{j}^{k}\right)^{-1} \leq\left.\operatorname{Lip} h_{k}\right|_{G_{j}^{k}} \circ\left(R_{j}^{k}\right)^{-1} \leq t,
\end{aligned}
$$

onde as últimas desigualdades em ambas as linhas são justificadas pelo fato de que $\left(G_{j}^{k}, R_{j}^{k}\right)$ é uma $t$-linearização para $h_{k}$ e pela proposição 3.2. Assim, $\forall k, j \in \mathbb{N}$, a condição i) no enunciado do lema é satisfeita por $\left(E_{j}^{k}, R_{j}^{k}\right)$.

7) $\forall k, j \in \mathbb{N}, \forall x \in E_{j}^{k}$, tem-se $h(x) \in G_{j}^{k} \cap Y_{k}$ e $x=h_{k}(h(x))$; em particular, pela definição de $Y_{k}$, $\mathrm{D} h(x) \circ \mathrm{D} h_{k}(h(x))=\mathrm{id}_{\mathbb{R}^{n}}$, i.e. $\mathrm{D} h(x)=\mathrm{D} h_{k}(h(x))^{-1}$. Então segue que, $\forall k, j \in \mathbb{N}, \forall x \in E_{j}^{k}$ :

$$
\begin{aligned}
\left\|\left(S_{j}^{k}\right)^{-1} \circ \mathrm{D} h(x)\right\| & =\left\|R_{j}^{k} \circ \mathrm{D} h_{k}(h(x))^{-1}\right\| \leq t, \\
\left\|\mathrm{D} h(x)^{-1} \circ S_{j}^{k}\right\| & =\left\|\mathrm{D} h_{k}(h(x)) \circ\left(R_{j}^{k}\right)^{-1}\right\| \leq t,
\end{aligned}
$$


onde as últimas desigualdades em ambas as linhas são justificadas pelo fato de que $\left(G_{j}^{k}, R_{j}^{k}\right)$ é uma $t$-linearização para $h_{k}$ e pela proposição 3.2. Assim, $\forall k, j \in \mathbb{N}$, a condição ii) no enunciado do lema é satisfeita por $\left(E_{j}^{k}, R_{j}^{k}\right)$, o que conclui a demonstração.

Lema 3.22. Sejam $n \leq m \leq k, T \in \mathrm{L}\left(\mathbb{R}^{n}, \mathbb{R}^{m}\right)$ e $R \in \mathrm{L}\left(\mathbb{R}^{m}, \mathbb{R}^{k}\right)$. Então $\llbracket R \circ T \rrbracket \leq\|R\|^{n} \llbracket T \rrbracket$. Além disso, se $R$ for $1-1$, então $\left\|R^{-1}\right\|^{-n} \llbracket T \rrbracket \leq \llbracket R \circ T \rrbracket \leq\|R\|^{n} \llbracket T \rrbracket$.

Demonstração. Denotando por $\mathbb{B}$ a bola fechada unitária em $\mathbb{R}^{k}$, segue do lema 3.6 que:

$$
\begin{aligned}
\llbracket R \circ T \rrbracket & \stackrel{3.6}{=}|\mathbb{B}|^{-1} \mathcal{H}^{n}(R \circ T(\mathbb{B})) \stackrel{2.49}{\leq} \\
& \leq|\mathbb{B}|^{-1}(\operatorname{Lip} R)^{n} \mathcal{H}^{n}(T(\mathbb{B})) \stackrel{3.6}{=} \\
& =\|R\|^{n} \llbracket T \rrbracket,
\end{aligned}
$$

o que prova a primeira afirmação.

Se $m=k$ e $R \in G L\left(\mathbb{R}^{m}\right)$, então $T=R^{-1} \circ R \circ T$; logo, pela primeira afirmação, $\llbracket T \rrbracket \leq$ $\left\|R^{-1}\right\|^{n} \llbracket R \circ T \rrbracket \Leftrightarrow \llbracket R \circ T \rrbracket \geq\left\|R^{-1}\right\|^{-n} \llbracket T \rrbracket$.

Se $m<k$ e $R$ 1-1, tomamos uma isometria linear $P: \operatorname{Im} R \rightarrow \mathbb{R}^{m}$, de modo que $\llbracket R \circ T \rrbracket=$ $\llbracket P \circ R \circ T \rrbracket,\|P \circ R\|=\|R\|$ e $\left\|(P \circ R)^{-1}\right\|=\left\|R^{-1}\right\|$. A tese segue, então, aplicando o caso já provado com $P \circ R \in \mathrm{GL}\left(\mathbb{R}^{m}\right)$ no lugar de $R$.

Teorema 3.23 (fórmula de coárea). Seja $f: \mathbb{R}^{n} \rightarrow \mathbb{R}^{m}$ Lipschitz, com $n \geq m$. Então, para cada $\mathcal{L}^{n}$-mensurável $A \subset \mathbb{R}^{n}$,

$$
\int_{A} \mathrm{~J} f \mathrm{~d} \mathcal{L}^{n}=\int_{\mathbb{R}^{m}} \mathcal{H}^{n-m}\left(A \cap f^{-1}\{y\}\right) \mathrm{d} \mathcal{L}^{m}(y) .
$$

Observação 3.24 .

1) Recorde que $N\left(\left.f\right|_{A}\right): y \mapsto \mathcal{H}^{n-m}\left(A \cap f^{-1}\{y\}\right)$ é $\mathcal{L}^{m}$-mensurável, pelo lema 3.19 , de modo que a integral no segundo membro da fórmula da coárea faz sentido.

2) Se $f: \mathbb{R}^{n} \equiv \mathbb{R}^{m} \times \mathbb{R}^{n-m} \rightarrow \mathbb{R}^{m}$ é a projeção no primeiro fator, tem-se $\mathrm{J} f \equiv 1$ e a fórmula da coárea se reduz ao teorema de Fubini-Tonelli 2.39. O caso geral pode ser interpretado, portanto, como uma generalização "curvilínea" do teorema de Fubini-Tonelli.

3) Se $n=m$, a fórmula da coárea coincide com a fórmula da área 3.9.

4) Tomando o boreliano $A:=\left(\mathbb{R}^{n} \backslash D_{f}\right) \cup J_{f}^{0}=\left\{x \in \mathbb{R}^{n} \mid \nexists \mathrm{D} f(x)\right.$ or $\left.\mathrm{J} f(x)=0\right\}$ na fórmula da coárea, conclui-se que $\mathcal{H}^{n-m}\left(A \cap f^{-1}\{y\}\right)=0$ para $\mathcal{L}^{m}$-q.t. $y \in \mathbb{R}^{m}$. Isso pode ser interpretado como uma versão do teorema de Morse-Sard para a teoria da medida: $\mathcal{L}^{m}$-q.t. $y \in \mathbb{R}^{m}$ é um "valor regular" de $f$, no sentido de que, a menos de conjuntos $\mathcal{H}^{n-m}$ nulos, $f^{-1}\{y\}$ está no conjunto $J_{f}^{+}$dos pontos onde $\mathrm{D} f$ tem posto máximo.

Notação. Seja $n \leq m$.

1) Denotamos por:

- $\Phi(m, n)$ o conjunto de todas as aplicações $\{1, \ldots, n\} \rightarrow\{1, \ldots, m\}$.

- $\Sigma(m, n):=\{\lambda \in \Phi(m, n) \mid \lambda 1-1\}$. Abreviamos $\Sigma_{n}:=\Sigma(n, n)$ (i.e o conjunto das permutações de $\{1, \ldots, n\})$.

- $\Lambda(m, n):=\{\lambda \in \Sigma(m, n) \mid \lambda$ estritamente crescente $\}$.

2) Para $\lambda \in \Lambda(m, n)$, seja $S_{\lambda}:=\left\langle e_{\lambda(i)} \mid 1 \leq i \leq n\right\rangle \subset \mathbb{R}^{m}$ e $P_{\lambda} \in \mathrm{L}\left(\mathbb{R}^{m}, S_{\lambda}\right)$ a projeção ortogonal sobre $S_{\lambda}$, i.e. $P_{\lambda}\left(x_{1}, \ldots, x_{m}\right):=\left(x_{\lambda(1)}, \ldots, x_{\lambda(n)}\right)$. 


\section{Demonstração.}

1) Se $A \subset \mathbb{R}^{n} \backslash D_{f}$, então $\mathcal{L}^{n}(A)=0$ pelo teorema de Rademacher 2.97 , logo o primeiro membro em (3.17) é nulo, e o segundo membro idem em vista do lema $3.18 \mathrm{com} k=n-m$ e $l=m$. Assim, é suficiente mostrar (3.17) para $A \subset D_{f}=J_{f}^{+} \dot{\cup} J_{f}^{0}$. E, como ambos os membros são aditivos em $\sigma\left(\mathcal{L}^{n}\right)$, é suficiente considerar os casos $A \subset J_{f}^{+}$e $A \subset J_{f}^{0}$.

2) Caso 1: $A \subset J_{f}^{+}$. Para cada $\lambda \in \Lambda(n, n-m)$, defina $h_{\lambda}: \mathbb{R}^{n} \rightarrow \mathbb{R}^{m} \times \mathbb{R}^{n-m}$ por

$$
h_{\lambda}(x):=\left(f(x), P_{\lambda}(x)\right)
$$

onde $P_{\lambda}$ é a projeção ortogonal sobre $\left\langle e_{\lambda(1)}, \ldots, e_{\lambda(n-m)}\right\rangle \equiv \mathbb{R}^{n-m}$. Para todo $x \in D_{h_{\lambda}}=D_{f}$, tem-se $\mathrm{D} h_{\lambda}(x)=\left(\mathrm{D} f(x), P_{\lambda}\right) \in \mathrm{L}\left(\mathbb{R}^{n}\right) ; \operatorname{logo}, \mathrm{J} h_{\lambda}(x)>0$ see $x \in J_{f}^{\lambda}$, onde

$$
J_{f}^{\lambda}:=\left\{x \in J_{f}^{+}\left|P_{\lambda}\right|_{\operatorname{ker} \mathrm{D} f(x)} \text { is } 1-1\right\} .
$$

Para cada $x \in J_{f}^{+}$, existe $\lambda \in \Lambda(n, n-m)$ tal que ker $\mathrm{D} f(x)$ é transversal a $\left\langle e_{\lambda(1)}, \ldots, e_{\lambda(n-m)}\right\rangle \equiv$ $\mathbb{R}^{n-m}\left(\operatorname{logo} x \in J_{f}^{\lambda}\right)$. Ou seja, $J_{f}^{+}=\cup_{\lambda \in \Lambda(n, n-m)} J_{f}^{\lambda}$. Portanto, podemos decompor $A$ numa união disjunta $A=\dot{\cup}_{\lambda \in \Lambda(n, n-m)} A_{\lambda}$, com $A_{\lambda} \subset J_{f}^{\lambda} \mathcal{L}^{n}$-mensurável. Então, pela aditividade de ambos os membros de (3.17) em $\mathcal{L}^{n}$, é suficiente mostrar a igualdade para cada $A_{\lambda}$. Assim, podemos assumir que $A \subset J_{f}^{\lambda}=J_{h_{\lambda}}^{+}$para um dado $\lambda \in \Lambda(n, n-m)$.

3) Por simplicidade de notação, pomos $h:=h_{\lambda}: \mathbb{R}^{n} \rightarrow \mathbb{R}^{n}$. Seja $q: \mathbb{R}^{n} \equiv \mathbb{R}^{m} \times \mathbb{R}^{n-m} \rightarrow \mathbb{R}^{m}$ a projeção no primeiro fator, de modo que $f=q \circ h$.

Fixe $t>1$. Aplique o lema 3.20 para $h$ de forma a obter uma família enumerável disjunta $\left(E_{k}\right)_{k \in \mathbb{N}}$ in $\mathscr{B}_{J_{h}^{+}}$e uma sequência $\left(S_{k}\right)_{k \in \mathbb{N}} \in \operatorname{Sym}(n) \cap \operatorname{GL}\left(\mathbb{R}^{n}\right)$ tais que $\mathcal{L}^{n}\left(J_{h}^{+} \backslash \dot{\cup}_{k \in \mathbb{N}} E_{k}\right)=0$ e, $\forall k \in \mathbb{N},\left.h\right|_{E_{k}}$ é 1-1 e as condições i), ii) no enunciado do referido lema são satisfeitas. Seja, $\forall k \in \mathbb{N}, A_{k}:=A \cap E_{k} \in \sigma\left(\mathcal{L}^{n}\right)$, de modo que $A \backslash \dot{\cup}_{k \in \mathbb{N}} A_{k}$ é $\mathcal{L}^{n}$-nulo (pois $A \subset J_{h}^{+}$).

Afirmamos que, $\forall k \in \mathbb{N}, \forall x \in A_{k}$,

$$
t^{-m} \llbracket q \circ S_{k} \rrbracket \leq \mathrm{J} f(x) \leq t^{m} \llbracket q \circ S_{k} \rrbracket .
$$

De fato, como $f=q \circ h, \mathrm{D} f(x)=q \circ \mathrm{D} h(x)=q \circ S_{k} \circ\left(S_{k}^{-1} \circ \mathrm{D} h(x)\right)$. Logo, definindo $C:=$ $S_{k}^{-1} \circ \mathrm{D} h(x)$, tem-se $q \circ S_{k}=\mathrm{D} f(x) \circ C^{-1}$, donde $\left(q \circ S_{k}\right)^{*}=\left(C^{-1}\right)^{*} \circ \mathrm{D} f(x)^{*}$. Portanto, aplicando o lema 3.22 com $\mathrm{D} f(x)^{*}: \mathbb{R}^{m} \rightarrow \mathbb{R}^{n}$ no lugar de $T$ e $\left(C^{-1}\right)^{*} \in \mathrm{GL}\left(\mathbb{R}^{n}\right)$ no lugar de $R$, e notando que $\left(C^{-1}\right)^{*}=\left(C^{*}\right)^{-1}$ e que a operação de transposição $(\cdot)^{*}$ preserva jacobianos e é uma isometria linear, conclui-se que

$$
\|C\|^{-m} \mathrm{~J} f(x) \leq \llbracket q \circ S_{k} \rrbracket \leq\left\|C^{-1}\right\|^{m} \mathrm{~J} f(x),
$$

$\log 0$

$$
\begin{aligned}
& \mathrm{J} f(x) \leq\|C\|^{m} \llbracket q \circ S_{k} \rrbracket \stackrel{i i) \text { pelo lema } 3.20}{\leq} t^{m} \llbracket q \circ S_{k} \rrbracket, \\
& \mathrm{J} f(x) \geq\left\|C^{-1}\right\|^{-m} \llbracket q \circ S_{k} \rrbracket \stackrel{\text { ii) pelo lema } 3.20}{\geq} t^{-m} \llbracket q \circ S_{k} \rrbracket,
\end{aligned}
$$

o que conclui a prova da afirmação. 
4) $\forall k \in \mathbb{N}$,

$$
\begin{aligned}
& t^{-2 n} \int_{\mathbb{R}^{m}} \mathcal{H}^{n-m}\left(A_{k} \cap f^{-1}\{y\}\right) \mathrm{d} \mathcal{L}^{m}(y)= \\
& =t^{-2 n} \int_{\mathbb{R}^{m}} \mathcal{H}^{n-m}\left(\left.\left(\left.S_{k}^{-1} \circ h\right|_{A_{k}}\right)^{-1} \circ S_{k}^{-1} \circ h\right|_{A_{k}}\left(A_{k} \cap h^{-1} q^{-1}\{y\}\right)\right) \mathrm{d} \mathcal{L}^{m}(y)= \\
& =t^{-2 n} \int_{\mathbb{R}^{m}} \mathcal{H}^{n-m}\left(\left(\left.S_{k}^{-1} \circ h\right|_{A_{k}}\right)^{-1}\left[S_{k}^{-1} \circ h\left(A_{k}\right) \cap\left(q \circ S_{k}\right)^{-1}\{y\}\right]\right) \mathrm{d} \mathcal{L}^{m}(y) \stackrel{2.49 . i i i)}{\leq} \\
& \leq\left[\operatorname{Lip}\left(\left.h\right|_{A_{k}}\right)^{-1} \circ S_{k}\right]^{n-m} t^{-2 n} \int_{\mathbb{R}^{m}} \mathcal{H}^{n-m}\left(S_{k}^{-1} \circ h\left(A_{k}\right) \cap\left(q \circ S_{k}\right)^{-1}\{y\}\right) \mathrm{d} \mathcal{L}^{m}(y) \stackrel{3.20 . i)}{\leq} \\
& \leq t^{-n-m} \int_{\mathbb{R}^{m}} \mathcal{H}^{n-m}\left(S_{k}^{-1} \circ h\left(A_{k}\right) \cap\left(q \circ S_{k}\right)^{-1}\{y\}\right) \mathrm{d} \mathcal{L}^{m}(y) \stackrel{3.17}{=} \\
& =t^{-n-m} \llbracket q \circ S_{k} \rrbracket \mathcal{L}^{n}\left(S_{k}^{-1} \circ h\left(A_{k}\right)\right) \stackrel{2.49 . i i i)}{\leq} \\
& \leq t^{-n-m} \llbracket q \circ S_{k} \rrbracket\left(\left.\operatorname{Lip} S_{k}^{-1} \circ h\right|_{A_{k}}\right)^{n} \mathcal{L}^{n}\left(A_{k}\right) \stackrel{3.20 . i)}{\leq} \\
& \leq t^{-m} \llbracket q \circ S_{k} \rrbracket \mathcal{L}^{n}\left(A_{k}\right) \stackrel{(3.18)}{\leq} \\
& \leq \int_{A_{k}} J f \mathrm{~d} \mathcal{L}^{n} \stackrel{(3.18)}{\leq} \\
& \leq t^{m} \llbracket q \circ S_{k} \rrbracket \mathcal{L}^{n}\left(A_{k}\right)=t^{m} \llbracket q \circ S_{k} \rrbracket \mathcal{L}^{n}\left(\left(\left.h\right|_{A_{k}} ^{-1} \circ S_{k}\right) \circ\left(\left.S_{k}^{-1} \circ h\right|_{A_{k}}\right)\left(A_{k}\right)\right) \stackrel{2.49 . i i i)}{\leq} \\
& \leq t^{m} \llbracket q \circ S_{k} \rrbracket\left(\left.\operatorname{Lip} h\right|_{A_{k}} ^{-1} \circ S_{k}\right)^{n} \mathcal{L}^{n}\left(\left.S_{k}^{-1} \circ h\right|_{A_{k}}\left(A_{k}\right)\right) \stackrel{3.20 . i)}{\leq} \\
& \leq t^{m+n} \llbracket q \circ S_{k} \rrbracket \mathcal{L}^{n}\left(\left.S_{k}^{-1} \circ h\right|_{A_{k}}\left(A_{k}\right)\right) \stackrel{3.17}{=} \\
& =t^{m+n} \int_{\mathbb{R}^{m}} \mathcal{H}^{n-m}\left(S_{k}^{-1} \circ h\left(A_{k}\right) \cap\left(q \circ S_{k}\right)^{-1}\{y\}\right) \mathrm{d} \mathcal{L}^{m}(y)= \\
& =t^{m+n} \int_{\mathbb{R}^{m}} \mathcal{H}^{n-m}\left[\left(\left.S_{k}^{-1} \circ h\right|_{A_{k}}\right) \circ\left(\left.h\right|_{A_{k}} ^{-1} \circ S_{k}\right)\left(S_{k}^{-1} \circ h\left(A_{k}\right) \cap\left(q \circ S_{k}\right)^{-1}\{y\}\right)\right] \mathrm{d} \mathcal{L}^{m}(y)= \\
& =t^{m+n} \int_{\mathbb{R}^{m}} \mathcal{H}^{n-m}\left[\left.S_{k}^{-1} \circ h\right|_{A_{k}}\left(\left.A_{k} \cap h\right|_{A_{k}} ^{-1} q^{-1}\{y\}\right)\right] \mathrm{d} \mathcal{L}^{m}(y)= \\
& =t^{m+n} \int_{\mathbb{R}^{m}} \mathcal{H}^{n-m}\left[\left.S_{k}^{-1} \circ h\right|_{A_{k}}\left(A_{k} \cap f^{-1}\{y\}\right)\right] \mathrm{d} \mathcal{L}^{m}(y) \stackrel{2.49 . i i i)}{\leq} \\
& \leq t^{m+n}\left(\left.\operatorname{Lip} S_{k}^{-1} \circ h\right|_{A_{k}}\right)^{n-m} \int_{\mathbb{R}^{m}} \mathcal{H}^{n-m}\left(A_{k} \cap f^{-1}\{y\}\right) \mathrm{d} \mathcal{L}^{m}(y) \stackrel{3.20 . i)}{\leq} \\
& \leq t^{2 n} \int_{\mathbb{R}^{m}} \mathcal{H}^{n-m}\left(A_{k} \cap f^{-1}\{y\}\right) \mathrm{d} \mathcal{L}^{m}(y) .
\end{aligned}
$$

Em particular, $\forall k \in \mathbb{N}$,

$$
\begin{aligned}
& t^{-2 n} \int_{\mathbb{R}^{m}} \mathcal{H}^{n-m}\left(A_{k} \cap f^{-1}\{y\}\right) \mathrm{d} \mathcal{L}^{m}(y) \leq \int_{A_{k}} J f \mathrm{~d} \mathcal{L}^{n} \leq \\
& \leq t^{2 n} \int_{\mathbb{R}^{m}} \mathcal{H}^{n-m}\left(A_{k} \cap f^{-1}\{y\}\right) \mathrm{d} \mathcal{L}^{m}(y) .
\end{aligned}
$$

5) Segue do lema 3.19 que, para $\mathcal{L}^{n}$-q.t. $y \in \mathbb{R}^{m}, \forall k \in \mathbb{N}, A_{k} \cap f^{-1}\{y\}$ é $\mathcal{H}^{n-m}$-mensurável, logo $\mathcal{H}^{n-m}\left(\dot{\cup}_{k \in \mathbb{N}} A_{k} \cap f^{-1}\{y\}\right)=\sum_{k \in \mathbb{N}} \mathcal{H}^{n-m}\left(A_{k} \cap f^{-1}\{y\}\right)$. Daí segue do teorema da convergência monótona que

$$
\int_{\mathbb{R}^{m}} \mathcal{H}^{n-m}\left(\cup_{k \in \mathbb{N}} A_{k} \cap f^{-1}\{y\}\right) \mathrm{d} \mathcal{L}^{m}(y)=\sum_{k \in \mathbb{N}} \int_{\mathbb{R}^{m}} \mathcal{H}^{n-m}\left(A_{k} \cap f^{-1}\{y\}\right) \mathrm{d} \mathcal{L}^{m}(y) .
$$


6) Afirmamos que

$$
\int_{\mathbb{R}^{m}} \mathcal{H}^{n-m}\left(\underset{k \in \mathbb{N}}{\dot{\cup}} A_{k} \cap f^{-1}\{y\}\right) \mathrm{d} \mathcal{L}^{m}(y)=\int_{\mathbb{R}^{m}} \mathcal{H}^{n-m}\left(A \cap f^{-1}\{y\}\right) \mathrm{d} \mathcal{L}^{m}(y) .
$$

Da fato, como $\dot{\cup}_{k \in \mathbb{N}} A_{k} \subset A$, a desigualdade " $\leq$ " vale trivialmente em (3.21). Por outro lado, por subaditividade, tem-se, $\forall y \in \mathbb{R}^{m}, \mathcal{H}^{n-m}\left(A \cap f^{-1}\{y\}\right) \leq \mathcal{H}^{n-m}\left(\dot{\cup}_{k \in \mathbb{N}} A_{k} \cap f^{-1}\{y\}\right)+$ $\mathcal{H}^{n-m}\left(\left(A \backslash \dot{\cup}_{k \in \mathbb{N}} A_{k}\right) \cap f^{-1}\{y\}\right)$, donde $\int_{\mathbb{R}^{m}} \mathcal{H}^{n-m}\left(A \cap f^{-1}\{y\}\right) \mathrm{d} \mathcal{L}^{m}(y) \leq \int_{\mathbb{R}^{m}} \mathcal{H}^{n-m}\left(\dot{\cup}_{k \in \mathbb{N}} A_{k} \cap\right.$ $\left.f^{-1}\{y\}\right) \mathrm{d} \mathcal{L}^{m}(y)+\int_{\mathbb{R}^{m}} \mathcal{H}^{n-m}\left(\left(A \backslash \dot{\cup}_{k \in \mathbb{N}} A_{k}\right) \cap f^{-1}\{y\}\right) \mathrm{d} \mathcal{L}^{m}(y)$. Como $A \backslash \dot{\cup}_{k \in \mathbb{N}} A_{k}$ é $\mathcal{L}^{n}$-nulo (pela parte 3), segue do lema 3.18 com $k=n-m$ e $l=m$ que $\int_{\mathbb{R}^{m}} \mathcal{H}^{n-m}\left(\left(A \backslash \dot{\cup}_{k \in \mathbb{N}} A_{k}\right) \cap\right.$ $\left.f^{-1}\{y\}\right) \mathrm{d} \mathcal{L}^{m}(y)=0$, assim provando a desigualdade oposta e a afirmação segue.

Então segue de (3.20) e (3.21) que

$$
\int_{\mathbb{R}^{m}} \mathcal{H}^{n-m}\left(A \cap f^{-1}\{y\}\right) \mathrm{d} \mathcal{L}^{m}(y)=\sum_{k \in \mathbb{N}} \int_{\mathbb{R}^{m}} \mathcal{H}^{n-m}\left(A_{k} \cap f^{-1}\{y\}\right) \mathrm{d} \mathcal{L}^{m}(y) .
$$

7) Como $A \backslash \dot{\cup}_{k \in \mathbb{N}} A_{k}$ é $\mathcal{L}^{n}$-nulo, tem-se

$$
\int_{A} \mathrm{~J} f \mathrm{~d} \mathcal{L}^{n}=\int_{\dot{\cup}_{k \in \mathbb{N}} A_{k}} \mathrm{~J} f \mathrm{~d} \mathcal{L}^{n} \stackrel{\mathrm{TCM}}{=} \sum_{k \in \mathbb{N}} \int_{A_{k}} \mathrm{~J} f \mathrm{~d} \mathcal{L}^{n} .
$$

Finalmente, de (3.23), (3.22) e (3.19), conclui-se que

$$
\begin{aligned}
& t^{-2 n} \int_{\mathbb{R}^{m}} \mathcal{H}^{n-m}\left(A \cap f^{-1}\{y\}\right) \mathrm{d} \mathcal{L}^{m}(y) \leq \int_{A} J f \mathrm{~d} \mathcal{L}^{n} \leq \\
& \leq t^{2 n} \int_{\mathbb{R}^{m}} \mathcal{H}^{n-m}\left(A \cap f^{-1}\{y\}\right) \mathrm{d} \mathcal{L}^{m}(y) .
\end{aligned}
$$

Como $t>1$ foi tomado arbitrariamente, podemos fazer $t \downarrow 1$ para obter

$$
\int_{\mathbb{R}^{m}} \mathcal{H}^{n-m}\left(A \cap f^{-1}\{y\}\right) \mathrm{d} \mathcal{L}^{m}(y)=\int_{A} J f \mathrm{~d} \mathcal{L}^{n},
$$

o que conclui a prova do caso 1.

8) Caso 2: $A \subset J_{f}^{0}$.

Note que ambos os membros em (3.17) são $\sigma$-aditivos em $\sigma\left(\mathcal{L}^{n}\right)$ (para o segundo membro, isso é uma consequência do lema 3.19 e do teorema da convergência monótona). Assim, pela $\sigma$-finitude de $\mathcal{L}^{n}$, podemos assumir que $\mathcal{L}^{n}(A)<\infty$.

Fixe $\epsilon>0$ e defina $g: \mathbb{R}^{n} \times \mathbb{R}^{m} \rightarrow \mathbb{R}^{m}$ por $g(x, y):=f(x)+\epsilon y, q: \mathbb{R}^{n} \times \mathbb{R}^{m} \rightarrow \mathbb{R}^{n} \mathrm{e}$ $p: \mathbb{R}^{n} \times \mathbb{R}^{m} \rightarrow \mathbb{R}^{m}$ as projeções no primeiro e segundo fatores, respectivamente. Então $g$ é Lipschitz e, $\forall(x, y) \in D_{g}=D_{f} \times \mathbb{R}^{m}, \mathrm{D} g(x, y)=\mathrm{D} f(x) \circ q+\epsilon p$. Portanto, a transposta da matriz jacobiana $[\mathrm{D} g(x, y)]$ é a $(n+m) \times m$ matriz que se escreve em blocos como

$$
\left[\mathrm{D} g(x, y)^{*}\right]=\left(\begin{array}{c}
{\left[\mathrm{D} f(x)^{*}\right]} \\
\epsilon I_{m}
\end{array}\right)
$$

i.e. é da mesma forma que (3.8), trocando $m$ com $n$. Como a $i$-ésima linha da matriz [D $\left.f(x)^{*}\right]$ é a $i$-ésima derivada parcial de $f$ em $x$, i.e. $\operatorname{D~} f(x) \cdot e_{i}$, a norma da referida linha é $\leq \operatorname{Lip} f$. Portanto, pelo mesmo argumento usado na página 32 (caso 2 da fórmula da área), i.e. usando a 
fórmula de Binet-Cauchy 2.104, conclui-se que, $\forall(x, y) \in D_{g}=D_{f} \times \mathbb{R}^{m}$,

$$
\begin{aligned}
\mathrm{J} g(x, y) & \geq \epsilon^{m}>0, \\
(\mathrm{~J} g(x, y))^{2} & \leq(\mathrm{J} f(x))^{2}+\left(\left(\begin{array}{c}
n+m \\
m
\end{array}\right)-\left(\begin{array}{c}
n \\
m
\end{array}\right)\right) \epsilon^{2} \cdot \max \left\{1,(\operatorname{Lip} f)^{m-1}\right\}^{2} .
\end{aligned}
$$

Em particular, se $(x, y) \in A \times \mathbb{R}^{m} \subset J_{f}^{0} \times \mathbb{R}^{m}$, podemos concluir que $\mathrm{J} g(x, y) \leq C \epsilon$, onde

$$
C:=\sqrt{\left(\begin{array}{c}
n+m \\
m
\end{array}\right)-\left(\begin{array}{c}
n \\
m
\end{array}\right)} \max \left\{1,(\operatorname{Lip} f)^{m-1}\right\} .
$$

Logo, $\forall(x, y) \in A \times \mathbb{R}^{m}, 0<J g(x, y) \leq C \epsilon$; em particular, $A \times \mathbb{R}^{m} \subset J_{g}^{+}$, e assim podemos aplicar o caso 1 com $g$ no lugar de $f$ e qualquer subconjunto $\mathcal{L}^{n+m}$-mensurável de $A \times \mathbb{R}^{m}$ no lugar de $A$.

9) Recorde que, pelo lema 3.19, a aplicação $N\left(\left.f\right|_{A}\right): \mathbb{R}^{m} \rightarrow[0, \infty]$ dada por $N\left(\left.f\right|_{A}\right)(y)=\mathcal{H}^{n-m}(A \cap$ $\left.f^{-1}\{y\}\right)$ é $\mathcal{L}^{m}$-mensurável. Como $\eta: \mathbb{R}^{m} \times \mathbb{R}^{m} \rightarrow \mathbb{R}^{m}$ dada por $\eta(y, w):=y-\epsilon w$ é linear e sobrejetiva, ela é mensurável como aplicação $\left(\mathbb{R}^{2 m} \equiv \mathbb{R}^{m} \times \mathbb{R}^{m}, \mathscr{L}_{\mathbb{R}^{2 m}}\right) \rightarrow\left(\mathbb{R}^{m}, \mathscr{L}_{\mathbb{R}^{m}}\right)$ (uma vez que pode ser fatorada como $\eta=q \circ \psi$, onde $\psi \in \mathrm{GL}\left(\mathbb{R}^{2 m}\right)$ e $q: \mathbb{R}^{m} \times \mathbb{R}^{m} \rightarrow \mathbb{R}^{m}$ é a projeção no primeiro fator, e isomorfismos lineares preservam a $\sigma$-álgebra de Lebesgue). Portanto, a composta $N\left(\left.f\right|_{A}\right) \circ \eta$ é $\geq 0$ e $\mathcal{L}^{2 m}$-mensurável, e também o é a aplicação $\mathbb{R}^{m} \times \mathbb{R}^{m} \rightarrow[0, \infty]$ dada por $(y, w) \mapsto \chi_{\mathbb{B}(0,1)}(w) \cdot N\left(\left.f\right|_{A}\right)(y-\epsilon w)$. Como $\mathcal{L}^{2 m}=\mathcal{L}^{m} \times \mathcal{L}^{m}$ (vide exemplo 2.41), isso justifica a aplicação do teorema de Fubini-Tonelli 2.39 no cálculo abaixo:

$$
\begin{aligned}
\forall w & \in \mathbb{R}^{m}, \int_{\mathbb{R}^{m}} \mathcal{H}^{n-m}\left(A \cap f^{-1}\{y\}\right) \mathrm{d} \mathcal{L}^{m}(y)= \\
& =\int_{\mathbb{R}^{m}} \mathcal{H}^{n-m}\left(A \cap f^{-1}\{y-\epsilon w\}\right) \mathrm{d} \mathcal{L}^{m}(y)= \\
& =\frac{1}{\alpha(m)} \int_{\mathbb{B}(0,1)} \int_{\mathbb{R}^{m}} \mathcal{H}^{n-m}\left(A \cap f^{-1}\{y-\epsilon w\}\right) \mathrm{d} \mathcal{L}^{m}(y) \mathrm{d} \mathcal{L}^{m}(w)= \\
& =\frac{1}{\alpha(m)} \int_{\mathbb{R}^{m}} \int_{\mathbb{R}^{m}} \chi_{\mathbb{B}(0,1)}(w) \cdot \mathcal{H}^{n-m}\left(A \cap f^{-1}\{y-\epsilon w\}\right) \mathrm{d} \mathcal{L}^{m}(y) \mathrm{d} \mathcal{L}^{m}(w) \stackrel{\text { Fubini } 2.39}{=} \\
& =\frac{1}{\alpha(m)} \int_{\mathbb{R}^{m}} \int_{\mathbb{R}^{m}} \chi_{\mathbb{B}(0,1)}(w) \cdot \mathcal{H}^{n-m}\left(A \cap f^{-1}\{y-\epsilon w\}\right) \mathrm{d} \mathcal{L}^{m}(w) \mathrm{d} \mathcal{L}^{m}(y)= \\
& =\frac{1}{\alpha(m)} \int_{\mathbb{R}^{m}} \int_{\mathbb{R}^{m}} \chi_{\mathbb{B}(0,1)}(w) \cdot \mathcal{H}^{n-m}\left(\left(A \cap f^{-1}\{y-\epsilon w\}\right) \times\{w\}\right) \mathrm{d} \mathcal{L}^{m}(w) \mathrm{d} \mathcal{L}^{m}(y),
\end{aligned}
$$

onde a última igualdade se deve à proposição 2.49 aplicada à isometria $\mathbb{R}^{n} \rightarrow \mathbb{R}^{n} \times \mathbb{R}^{m}$ dada por $x \mapsto(x, w)$.

10) Note que, se $x \in \mathbb{R}^{n}$ e $w, y \in \mathbb{R}^{m}$, tem-se $(x \in A$ e $g(x, w)=y)$ see $(x \in A$ e $f(x)=y-\epsilon w)$ see $x \in A \cap f^{-1}\{y-\epsilon w\}$. Logo, definindo $B:=A \times \mathbb{B}(0,1) \subset \mathbb{R}^{n} \times \mathbb{R}^{m}$, vale a seguinte igualdade:

$$
B \cap g^{-1}\{y\} \cap p^{-1}\{w\}= \begin{cases}\emptyset & \text { se } w \notin \mathbb{B}(0,1) \\ \left(A \cap f^{-1}\{y-\epsilon w\}\right) \times\{w\} & \text { se } w \in \mathbb{B}(0,1) .\end{cases}
$$

Então, $\forall(y, w) \in \mathbb{R}^{m} \times \mathbb{R}^{m}$,

$$
\chi_{\mathbb{B}(0,1)}(w) \cdot \mathcal{H}^{n-m}\left(\left(A \cap f^{-1}\{y-\epsilon w\}\right) \times\{w\}\right)=\mathcal{H}^{n-m}\left(B \cap g^{-1}\{y\} \cap p^{-1}\{w\}\right) .
$$


Portanto, segue de (3.24) que

$$
\begin{aligned}
& \int_{\mathbb{R}^{m}} \mathcal{H}^{n-m}\left(A \cap f^{-1}\{y\}\right) \mathrm{d} \mathcal{L}^{m}(y)= \\
& \quad=\frac{1}{\alpha(m)} \int_{\mathbb{R}^{m}} \int_{\mathbb{R}^{m}} \mathcal{H}^{n-m}\left(B \cap g^{-1}\{y\} \cap p^{-1}\{w\}\right) \mathrm{d} \mathcal{L}^{m}(w) \mathrm{d} \mathcal{L}^{m}(y) .
\end{aligned}
$$

Para continuar este cálculo, aplicamos o lema 3.18 à integral interna, com $B \cap g^{-1}\{y\} \in \sigma\left(\mathcal{L}^{n+m}\right)$ no lugar de $A, p: \mathbb{R}^{n} \times \mathbb{R}^{m} \rightarrow \mathbb{R}^{m}$ no lugar de $f, k=n-m$ e $l=m$, o que resulta em

$$
\begin{aligned}
\int_{\mathbb{R}^{m}} & \mathcal{H}^{n-m}\left(A \cap f^{-1}\{y\}\right) \mathrm{d} \mathcal{L}^{m}(y) \leq \\
= & \frac{1}{\alpha(m)} \frac{\alpha(n-m) \alpha(m)}{\alpha(n)} \int_{\mathbb{R}^{m}} \mathcal{H}^{n}\left(B \cap g^{-1}\{y\}\right) \mathrm{d} \mathcal{L}^{m}(y) \stackrel{\text { pelo caso } 1}{=} \\
= & \frac{\alpha(n-m)}{\alpha(n)} \int_{B} \mathrm{~J} g \mathrm{~d} \mathcal{L}^{n+m} \stackrel{\text { parte } 8}{\leq} \\
\leq & \frac{\alpha(n-m)}{\alpha(n)} C \epsilon \cdot \mathcal{L}^{n+m}(B)= \\
= & \frac{\alpha(n-m) \alpha(m)}{\alpha(n)} C \epsilon \cdot \mathcal{L}^{n}(A) .
\end{aligned}
$$

Como $\mathcal{L}^{n}(A)<\infty$ (pela redução feita na primeira parte do passo 8) e $\epsilon>0$ foram tomados arbitrariamente, fazendo-se $\epsilon \downarrow 0$, obtém-se

$$
\int_{\mathbb{R}^{m}} \mathcal{H}^{n-m}\left(A \cap f^{-1}\{y\}\right) \mathrm{d} \mathcal{L}^{m}(y)=0=\int_{A} \mathrm{~J} f \mathrm{~d} \mathcal{L}^{n},
$$

o que conclui a prova do caso 2 e a tese segue.

Corolário 3.25 (teorema de Fubini-Tonelli curvilíneo). Seja $f: \mathbb{R}^{n} \rightarrow \mathbb{R}^{m}$ Lipschitz, com $n \geq m$. Então, para toda $g: \mathbb{R}^{n} \rightarrow \mathbb{R} \mathcal{L}^{n}$-mensurável com $g \geq 0$ ou g somável,

$$
\int_{\mathbb{R}^{n}} g \mathrm{~J} f \mathrm{~d} \mathcal{L}^{n}=\int_{\mathbb{R}^{m}}\left(\int_{f^{-1}\{y\}} g(x) \mathrm{d} \mathcal{H}^{n-m}(x)\right) \mathrm{d} \mathcal{L}^{m}(y),
$$

com o significado de que as integrais iteradas no segundo membro fazem sentido e vale a igualdade.

Demonstração. Suponha $g \geq 0$. Pela proposição 2.33, existe uma sequência $\left(A_{i}\right)_{i \in \mathbb{N}}$ em $\sigma\left(\mathcal{L}^{n}\right)$ tal que

$$
g=\sum_{i=1}^{\infty} \frac{1}{i} \chi_{A_{i}}
$$

Logo, para todo $y \in \mathbb{R}^{m}$,

$$
g \cdot \chi_{f^{-1}\{y\}}=\sum_{i=1}^{\infty} \frac{1}{i} \chi_{A_{i} \cap f^{-1}\{y\}} .
$$

Segue do lema 3.19 que, para $\mathcal{L}^{m}$-q.t. $y \in \mathbb{R}^{m}, \forall i \in \mathbb{N}, \chi_{A_{i} \cap f^{-1}\{y\}}$ é $\mathcal{H}^{n-m}$-mensurável; para um tal $y$, o teorema 2.31 garante que $g \cdot \chi_{f^{-1}\{y\}}$ é $\mathcal{H}^{n-m}$-mensurável e $\geq 0$, de modo que a integral interior no segundo membro de (3.25) faz sentido. Além disso, ainda para $y$ satisfazendo a condição acima, segue do teorema da convergência monótona que

$$
\int g(x) \cdot \chi_{f^{-1}\{y\}}(x) \mathrm{d} \mathcal{H}^{n-m}(x)=\sum_{i=1}^{\infty} \frac{1}{i} \mathcal{H}^{n-m}\left(A_{i} \cap f^{-1}\{y\}\right) .
$$


Como o segundo membro da igualdade acima define uma função $\mathcal{L}^{m}$-mensurável $\mathbb{R}^{m} \rightarrow[0, \infty]$ (em vista do lema 3.19 e do teorema 2.31), conclui-se que a função definida $\mathcal{L}^{m}$-q.s. por $y \mapsto$ $\int g(x) \cdot \chi_{f-1\{y\}}(x) \mathrm{d} \mathcal{H}^{n-m}(x)$ é $\mathcal{L}^{m}$-mensurável e

$$
\begin{aligned}
\int_{\mathbb{R}^{m}} & \left(\int_{f^{-1}\{y\}} g(x) \mathrm{d} \mathcal{H}^{n-m}(x)\right) \mathrm{d} \mathcal{L}^{m}(y)= \\
= & \int_{\mathbb{R}^{m}} \sum_{i=1}^{\infty} \frac{1}{i} \mathcal{H}^{n-m}\left(A_{i} \cap f^{-1}\{y\}\right) \mathrm{d} \mathcal{L}^{m}(y) \stackrel{\mathrm{TCM}}{=} \\
= & \sum_{i=1}^{\infty} \frac{1}{i} \int_{\mathbb{R}^{m}} \mathcal{H}^{n-m}\left(A_{i} \cap f^{-1}\{y\}\right) \mathrm{d} \mathcal{L}^{m}(y) \stackrel{3.23}{=} \\
= & \sum_{i=1}^{\infty} \frac{1}{i} \int \chi_{A_{i}} \mathrm{~J} f \mathrm{~d} \mathcal{L}^{n} \stackrel{\mathrm{T}}{=} \\
= & \int \sum_{i=1}^{\infty} \frac{1}{i} \chi_{A_{i}} \mathrm{~J} f \mathrm{~d} \mathcal{L}^{n}=\int_{\mathbb{R}^{n}} g \mathrm{~J} f \mathrm{~d} \mathcal{L}^{n},
\end{aligned}
$$

o que conclui a prova do caso em que $g \geq 0$. Para $g: \mathbb{R}^{n} \rightarrow \mathbb{R} \mathcal{L}^{n}$-somável, basta aplicar o caso que acabamos de provar para as partes positiva e negativa de $g$.

A exemplo da fórmula da área, a fórmula da coárea e o corolário acima continuam válidos se $f: \mathbb{R}^{n} \rightarrow \mathbb{R}^{m}$ for substituída por $f: \Omega \subset \mathbb{R}^{n} \rightarrow \mathbb{R}^{m}$ localmente Lipschitz, com $\Omega \subset \mathbb{R}^{n}$ aberto. Ou seja, vale o seguinte teorema:

Teorema 3.26 (fórmula da coárea para aplicações localmente Lipschitz). Sejam $n \geq m, \Omega \subset \mathbb{R}^{n}$ aberto e $f: \Omega \rightarrow \mathbb{R}^{m}$ localmente Lipschitz.

a) (fórmula da coárea) Para todo $\mathcal{L}^{n}$-mensurável $A \subset \Omega$,

- para $\mathcal{L}^{m}$-q.t. $y \in \mathbb{R}^{m}, f^{-1}\{y\} \cap A$ é $\mathcal{H}^{n-m}$-mensurável;

- a função $N\left(\left.f\right|_{A}\right): \mathbb{R}^{m} \rightarrow[0, \infty], y \mapsto \mathcal{H}^{n-m}\left(A \cap f^{-1}\{y\}\right)$, é $\mathcal{L}^{m}$-mensurável e

$$
\int_{A} \mathrm{~J} f \mathrm{~d} \mathcal{L}^{n}=\int_{\mathbb{R}^{m}} \mathcal{H}^{n-m}\left(A \cap f^{-1}\{y\}\right) \mathrm{d} \mathcal{L}^{m}(y) .
$$

b) (Fubini-Tonelli curvilíneo) Se $g: \Omega \rightarrow \mathbb{R}$ é $\left.\mathcal{L}^{n}\right|_{\Omega}$-mensurável e $g \geq 0$ ou $g \in \mathrm{L}^{1}\left(\left.\mathcal{L}^{n}\right|_{\Omega}\right)$, então

$$
\int_{\Omega} g \mathrm{~J} f \mathrm{~d} \mathcal{L}^{n}=\int_{\mathbb{R}^{m}}\left(\int_{f^{-1}\{y\}} g(x) \mathrm{d} \mathcal{H}^{n-m}(x)\right) \mathrm{d} \mathcal{L}^{n}(y),
$$

com o significado de que as integrais no segundo membro fazem sentido e vale a igualdade.

Como em 3.12, a prova se obtém combinando-se a versão já provada para aplicações Lipschitz 3.9 com o teorema de extensão de Kirszbraun 2.95 e com o teorema da convergência monótona.

Finalmente, a mesma observação feita em 3.13 pode ser repetida aqui: a fórmula da coárea vale, mais geralmente, para aplicações aproximadamente diferenciáveis no sentido de [9, 3.1.2], o que se prova como corolário da versão aqui provada para aplicações Lipschitz e do teorema de estrutura $[9,3.1 .8]$.

\subsubsection{Aplicações da Fórmula da Coárea}

Proposição 3.27 (integração em coordenadas polares). Se $g: \mathbb{R}^{n} \rightarrow \mathbb{R}$ é $\mathcal{L}^{n}$-mensurável e $g \geq 0$ ou $g \in \mathrm{L}^{1}\left(\mathcal{L}^{n}\right)$, então

$$
\int_{\mathbb{R}^{n}} g \mathrm{~d} \mathcal{L}^{n}=\int_{0}^{\infty}\left(\int_{\partial \mathbb{B}(0, r)} g \mathrm{~d} \mathcal{H}^{n-1}\right) \mathrm{d} r .
$$


Demonstração. Seja $f: \mathbb{R}^{n} \rightarrow \mathbb{R}$ dada por $f(x)=\|x\|$. Então $f$ é Lipschitz e, $\forall x \in D_{f}=\mathbb{R}^{n} \backslash\{0\}$, $\nabla f(x)=x /\|x\|, \operatorname{logo} \mathrm{J} f(x)=\|\nabla f(x)\|=1$. Como, $\forall r \in \mathbb{R}, f^{-1}\{r\}=\partial \mathbb{B}(0, r)$ (em particular, $=\emptyset$ para $r<0)$, (3.26) é uma consequência direta do corolário 3.25.

Proposição 3.28. Sejam $\Omega \subset \mathbb{R}^{n}$ aberto e $f: \Omega \rightarrow \mathbb{R}$ localmente Lipschitz. Então

$$
\int_{\Omega}\|\nabla f\| \mathrm{d} \mathcal{L}^{n}=\int_{-\infty}^{\infty} \mathcal{H}^{n-1}(\{f=t\}) \mathrm{d} t .
$$

Demonstração. É uma consequência direta do teorema 3.26.a), com $A=\Omega$, levando em conta que $\mathrm{J} f=\|\nabla f\|$. 


\section{Capítulo 4}

\section{Retificabilidade}

A noção de conjunto retificável, que fornece uma generalização da noção de superfície de importância primordial no estudo de problemas variacionais geométricos, será apresentada em nossa investigação em (4.1). As noções que usaremos neste capitulo seguem as formulações de F. Maggi [5], [17] e H. Federer [9].

Historicamente, conjuntos retificáveis foram introduzidos pela primeira vez por Besicovitch para conjuntos unidimensionais no plano em [1]. Em 1947, Federer escreveu um artigo sobre subconjuntos retificáveis do espaço $n$-dimensional e caracterizou os conjuntos puramente não retificáveis por sua "invisibilidade" sob quase todas as projeções (veja [11]). A.S. Besicovitch provou isso para conjuntos unidimensionais no plano, mas a generalização de Federer, válida para subconjuntos de dimensão arbitrária em qualquer espaço euclidiano, foi uma realização técnica importante e, mais tarde, desempenhou um papel fundamental na sua teoria de correntes normais e integrais.

\subsection{Conjuntos Retificáveis}

Recorde que, dado $k \in[0, \infty)$, usamos a notação $\mathcal{H}^{k}$ para denotar a medida de Hausdorff $k$ dimensional num espaço métrico, cf. def. 2.47, a qual é uma medida Borel-regular.

Definição 4.1 (conjuntos $k$-retificáveis em $\mathbb{R}^{n}$ ). Sejam $0 \leq k \leq n$ e $M \subset \mathbb{R}^{n} \mathcal{H}^{k}$-mensurável. Diz-se que $M$ é:

- $\mathcal{H}^{k}$-enumeravelmente retificável (ou k-enumeravelmente retificável) se existir uma sequência $\left(f_{i}\right)_{i \in \mathbb{N}}$ de aplicações Lipschitz $\mathbb{R}^{k} \rightarrow \mathbb{R}^{n}$ tal que

$$
\mathcal{H}^{k}\left(M \backslash \bigcup_{i \in \mathbb{N}} f_{i}\left(\mathbb{R}^{k}\right)\right)=0 .
$$

- localmente $k$-retificável se for $k$-enumeravelmente retificável e $\mathcal{H}^{k}\llcorner M$ for uma medida de Radon em $\mathbb{R}^{n}$.

- $k$-retificável se for $k$-enumeravelmente retificável e $\mathcal{H}^{k}\llcorner M$ for uma medida de Radon finita em $\mathbb{R}^{n}$.

Observação 4.2 .

1) A definição de Federer em [9, 3.2.14] é mais geral. No entanto, neste texto só será considerado o caso particular definido acima, a exemplo do que fazem [17] e [5].

2) Se $k=0, M \subset \mathbb{R}^{n}$ é $k$-enumeravelmente retificável se, e somente se, for um conjunto enumerável. Consideraremos, doravante, $k \geq 1$. 
3) Segue como consequência imediata da definição acima, do corolário 2.55 e da proposição 2.52 partes c) e e) que, se $M \subset \mathbb{R}^{n}$ for $k$-enumeravelmente retificável, então $\mathcal{H}$-dim $M \leq k$. Em particular, se $k<\kappa \leq n$ e $M$ for $k$-enumeravelmente retificável, também o será $\kappa$-enumeravelmente retificável, pois será $\mathcal{H}^{\kappa}$-nulo.

Proposição 4.3 (propriedades imediatas dos conjuntos $k$-retificáveis). Seja $M \subset \mathbb{R}^{n} \mathcal{H}^{k}$-mensurável.

i) Se $N \subset \mathbb{R}^{n}$ é tal que $\mathcal{H}^{k}(N \triangle M)=0$, então $N$ é $k$-enumeravelmente retificável (respectivamente, $k$-localmente retificável ou k-retificável) se, e somente se, $M$ o for.

ii) Todo subconjunto $\mathcal{H}^{k}$-mensurável de um conjunto $k$-enumeravelmente retificável é $k$-enumeravelmente retificável. A união de uma sequência enumerável de conjuntos $k$-enumeravelmente retificáveis é $k$-enumeravelmente retificável.

iii) $M$ é $k$-enumeravelmente retificável se, e somente se, existir uma sequência $\left(A_{i}\right)_{i \in \mathbb{N}}$ de subconjuntos limitados de $\mathbb{R}^{k}$ e uma sequência $\left\{f_{i}: A_{i} \rightarrow \mathbb{R}^{n}\right\}_{i \in \mathbb{N}}$ de aplicações Lipschitz tais que $M \backslash \bigcup_{i \in \mathbb{N}} f\left(A_{i}\right)$ é $\mathcal{H}^{k}$-nulo. Caso afirmativo, i.e. caso $M$ seja $k$-enumeravelmente retificável, é possivel tomar as $f_{i}$ 's definidas em $\mathbb{R}^{k}$ e os $A_{i}$ 's compactos.

iv) Se $M$ for $k$-enumeravelmente retificável, então $M$ é $\sigma$-finito com respeito a $\mathcal{H}^{k}$.

v) Se $M$ for $k$-enumeravelmente retificável, existe $N \supset \mathrm{M}$ boreliano tal que $\mathcal{H}^{k}(N \backslash M)=0$. Em particular, $\mathcal{H}^{k}\left\llcorner N=\mathcal{H}^{k}\left\llcorner M\right.\right.$, logo $\mathcal{H}^{k}\llcorner M$ é Borel-regular.

vi) $M$ é $k$-localmente retificável se, e somente se, for $k$-enumeravelmente retificável e $\mathcal{H}^{k}\llcorner M$ for finita nos compactos de $\mathbb{R}^{n}$.

Demonstração. i) É consequência imediata da definição.

ii) Idem.

iii) A implicação $(\Rightarrow)$ decorre do fato de que $\mathbb{R}^{k}$ se escreve como união enumerável de limitados. Para a implicação $(\Leftarrow)$, aplique o teorema de extensão de Kirszbraun 2.95 para cada $f_{i}: A_{i} \rightarrow$ $\mathbb{R}^{n}$. Caso $M$ seja retificável, podemos estender as $f_{i}$ 's da sequência a aplicações Lipschitz em $\mathbb{R}^{k}$ e substituir os $A_{i}$ 's pelos seus fechos, os quais são compactos.

iv) Em vista do item anterior, basta verificar que, se $A \subset \mathbb{R}^{k}$ for limitado e $f: A \rightarrow \mathbb{R}^{n}$ Lipschitz, então $\mathcal{H}^{k}(f(A))<\infty$. De fato, pela proposição 2.49.iii), segue $\mathcal{H}^{k}(f(A)) \leq(\operatorname{Lip} f)^{k} \mathcal{H}^{k}(A)$. Mas, pelo teorema 2.54 e pelo fato de ser $A \subset \mathbb{R}^{k}$ limitado, tem-se $\mathcal{H}^{k}(A)=\mathcal{L}^{k}(A)<\infty$, donde a tese.

v) Como $\mathcal{H}^{k}$ é Borel-regular, cf. proposição 2.49.v), o item anterior e a proposição 2.19 garantem a existência de um envoltório boreliano $N$ de $M$. Como $N \backslash M \in \sigma(\mu)$, segue da definição de envoltório 2.11 que $\mathcal{H}^{k}(N \backslash M)=\mathcal{H}^{k}((N \backslash M) \cap N)=\mathcal{H}^{k}((N \backslash M) \cap M)=\mathcal{H}^{k}(\emptyset)=0$. Segue daí que $\mathcal{H}^{k}\left\llcorner N=\mathcal{H}^{k}\left\llcorner M\right.\right.$, logo $\mathcal{H}^{k}\llcorner M$ é Borel-regular em vista da proposição 2.26.

vi) A implicação $(\Rightarrow)$ é imediata. A implicação $(\Leftarrow)$ decorre do item anterior e do fato de que toda medida Borel-regular localmente finita em $\mathbb{R}^{n}$ é de Radon, cf. observação 2.24.2).

Exemplo 4.4. Se $k \leq n$ e $\mathrm{M} \subset \mathbb{R}^{n}$ for uma subvariedade mergulhada ${ }^{1}$ de classe $\mathrm{C}^{1}$ e dimensão $k$, então $\mathrm{M}$ é $k$-enumeravelmente retificável e $\mathcal{H}$-dim $\mathrm{M}=k$. Com efeito:

1) Podemos cobrir $M$ com uma família enumerável $\left\{\varphi_{i}: \mathcal{U}_{i} \subset M \rightarrow \mathbb{R}^{k}\right\}_{i \in \mathbb{N}}$ de cartas $C^{1}$ tal que, para todo $i \in \mathbb{N}, \varphi_{i}: \mathcal{U}_{i} \rightarrow \mathbb{R}^{k}$ é bi-Lipschitz sobre sua imagem $\varphi_{i}\left(\mathcal{U}_{i}\right) \subset \mathbb{R}^{k}$. Então $\mathrm{M}$ se escreve como união enumerável das imagens das aplicações Lipschitz $\varphi_{i}^{-1}: \varphi_{i}\left(\mathcal{U}_{i}\right) \rightarrow \mathbb{R}^{n}, i \in \mathbb{N}, \operatorname{logo} \mathrm{M}$ é $k$-enumeravelmente retificável.

\footnotetext{
${ }^{1} \mathrm{ou}$, mais geralmente, e com o mesmo argumento, se $\mathrm{M}$ for a imagem de uma imersão de classe $\mathrm{C}^{1}$ definida numa variedade diferenciável de dimensão $k$.
} 
2) Do item anterior e da observação 4.2.3) segue que $\mathcal{H}$-dim $\mathrm{M} \leq k$. Por outro lado, tomando cartas $\left\{\varphi_{i}: \mathcal{U}_{i} \rightarrow \mathbb{R}^{k}\right\}_{i \in \mathbb{N}}$ como no item anterior, segue da proposição 2.52 que $\mathcal{H}$-dim $\mathrm{M} \geq \mathcal{H}$-dim $\mathcal{U}_{1}=$ $\mathcal{H}$-dim $\varphi_{1}\left(\mathcal{U}_{1}\right)=k$ (pois $\varphi_{1}$ é bi-Lipschitz sobre sua imagem $\varphi_{1}\left(\mathcal{U}_{1}\right)$, que é um aberto não vazio de $\left.\mathbb{R}^{k}\right)$. Portanto, $\mathcal{H}$-dim $\mathrm{M}=k$, como afirmado.

Além disso, se $\mathrm{M}$ for fechada em $\mathbb{R}^{n}$, então $\mathrm{M}$ é $k$-localmente retificável. Isso segue do item anterior e da proposição 4.3.vi), observando que a hipótese de ser $\mathrm{M}$ fechada e o fato de que $\left.\mathcal{H}^{k}\right|_{\mathrm{M}}$ é uma medida de Radon em $\mathrm{M}$, pelo teorema 3.15, garantem a finitude de $\mathcal{H}^{k}\llcorner\mathrm{M}$ nos compactos de $\mathbb{R}^{n}$.

Por outro lado, se $\mathrm{M}$ não for fechada em $\mathbb{R}^{n}$, $\mathrm{M}$ pode não ser $k$-localmente retificável, como mostra o exemplo, com $k=1$ e $\mathrm{M} \subset \mathbb{R}^{2}$ dada por $\mathrm{M}=\left\{\left(x, \sin \frac{1}{x}\right) \mid x>0\right\}$. Neste exemplo, $\mathrm{M}$ é uma subvariedade mergulhada de classe $C^{1}$ em $\mathbb{R}^{2}$, logo é 1-enumeravelmente retificável. No entanto, não é 1-localmente retificável, pois $\mathcal{H}^{1}\llcorner M$ não é localmente finita.

\subsection{Um Teorema de Estrutura para Conjuntos Retificáveis}

Nosso próximo objetivo é investigar uma decomposição canônica para conjuntos retificáveis, a qual será enunciada e provada no teorema de estrutura 4.7. Recorde a notação fixada em 3.1.

Definição 4.5 (imagem Lipschitz regular). Sejam $f: \mathbb{R}^{n} \rightarrow \mathbb{R}^{m}$ Lipschitz e $E \subset \mathbb{R}^{n}$ boreliano limitado. Diz-se que o par $(f, E)$ define uma imagem Lipschitz regular $f(E)$ em $\mathbb{R}^{m}$ se:

i) $f$ é 1-1 e diferenciável em $E$, com $\mathrm{J} f(x)>0$ em todo $x \in E$;

ii) todo $x \in E$ é ponto de densidade 1 para $E$, i.e.

$$
\lim _{r \rightarrow 0^{+}} \frac{|E \cap \mathbb{B}(x, r)|}{\alpha(n) r^{n}}=1
$$

iii) todo $x \in E$ é ponto de Lebesgue para $\mathrm{D} f: D_{f} \subset \mathbb{R}^{n} \rightarrow \mathrm{L}\left(\mathbb{R}^{n}, \mathbb{R}^{m}\right)$, i.e.

$$
\lim _{r \rightarrow 0^{+}} \frac{1}{\mathcal{L}^{n}(\mathbb{B}(x, r))} \int_{\mathbb{B}(x, r)}\|\mathrm{D} f(y)-\mathrm{D} f(x)\| \mathrm{d} \mathcal{L}^{n}(y)=0 .
$$

Proposição 4.6. Com a notação da definição acima, se $f(E)$ é uma imagem Lipschitz regular definida pelo par $(f, E)$, então todo $x \in E$ é ponto de Lebesgue para $\mathrm{J} f: D_{f} \rightarrow \mathbb{R}$.

Demonstração. Recorde a notação fixada em 2.102. Queremos provar que

$$
\lim _{r \rightarrow 0} \frac{1}{\alpha(n) r^{n}} \int_{\mathbb{B}(x, r)}|\llbracket \mathrm{D} f(y) \rrbracket-\llbracket \mathrm{D} f(x) \rrbracket| \mathrm{d} y=0 .
$$

Basta provar que toda sequência $\left(r_{i}\right)_{i \in \mathbb{N}}$ com $r_{i} \rightarrow 0$ admite uma subsequência $\left(r_{i_{j}}\right)_{j \in \mathbb{N}}$ tal que

$$
\lim _{j \rightarrow \infty} \frac{1}{\alpha(n) r_{i_{j}}^{n}} \int_{\mathbb{B}\left(x, r_{i_{j}}\right)}|\llbracket \mathrm{D} f(y) \rrbracket-\llbracket \mathrm{D} f(x) \rrbracket| \mathrm{d} y=0 .
$$

Por hipótese, $x$ é ponto de Lebesgue de $\mathrm{D} f$, ou seja,

$\frac{1}{\alpha(n) r_{i}^{n}} \int_{\mathbb{B}\left(x, r_{i}\right)}\|\mathrm{D} f(y)-\mathrm{D} f(x)\| \mathrm{d} y \stackrel{\mathrm{TMV}}{\operatorname{com} y=x+r_{i} w} \frac{1}{\alpha(n)} \int_{\mathbb{B}(0,1)}\left\|\mathrm{D} f\left(x+r_{i} w\right)-\mathrm{D} f(x)\right\| \mathrm{d} w \stackrel{i \rightarrow \infty}{\rightarrow} 0$.

Portanto, a sequência de funções $\left\{w \mapsto\left\|\mathrm{D} f\left(x+r_{i} w\right)-\mathrm{D} f(x)\right\|\right\}_{i \in \mathbb{N}}$ converge para $0 \mathrm{em} \mathrm{L}^{1}(\mathbb{B}(0,1))$. Existe, pois, uma subsequência da referida sequência que converge a $0 \mathcal{L}^{n}$-q.s. em $\mathbb{B}(0,1)$. Ou seja, existe $\left(r_{i_{j}}\right)_{j \in \mathbb{N}}$ subsequência de $\left(r_{i}\right)_{i \in \mathbb{N}}$ tal que, para $\mathcal{L}^{n}$-q.t. $w \in \mathbb{B}(0,1),\left\{\mathrm{D} f\left(x+r_{i_{j}} w\right)\right\}_{j \in \mathbb{N}}$ 
converge para $\mathrm{D} f(x)$. Como a aplicação $\llbracket \cdot \rrbracket: \mathrm{L}\left(\mathbb{R}^{n}, \mathbb{R}^{m}\right) \rightarrow \mathbb{R}$ é contínua, conclui-se que, para $\mathcal{L}^{n}$-q.t. $w \in \mathbb{B}(0,1)$,

$$
\left|\llbracket \mathrm{D} f\left(x+r_{i_{j}} w\right) \rrbracket-\llbracket \mathrm{D} f(x) \rrbracket\right| \stackrel{j \rightarrow \infty}{\rightarrow} 0 .
$$

Além disso, a convergência é dominada (pois, para todo $j \in \mathbb{N},\left|\llbracket \mathrm{D} f\left(x+r_{i_{j}} w\right) \rrbracket-\llbracket \mathrm{D} f(x) \rrbracket\right| \leq$ $\left.2(\operatorname{Lip} f)^{n}\right)$, o que nos permite concluir que

$$
\frac{1}{\alpha(n) r_{i_{j}}^{n}} \int_{\mathbb{B}\left(x, r_{i}\right)}|\mathrm{J} f(y)-\mathrm{J} f(x)| \mathrm{d} y \stackrel{y=x+r_{i_{j}} w}{=} \frac{1}{\alpha(n)} \int_{\mathbb{B}(0,1)}\left|\llbracket \mathrm{D} f\left(x+r_{i_{j}} w\right) \rrbracket-\llbracket \mathrm{D} f(x) \rrbracket\right| \mathrm{d} w \stackrel{j \rightarrow \infty}{\rightarrow} 0,
$$

pelo teorema da convergência dominada.

Teorema 4.7 (decomposição de conjuntos retificáveis). Sejam $M \subset \mathbb{R}^{n}$ um conjunto $k$-enumeravelmente retificável e $t>1$. Então existem $M_{0} \subset \mathbb{R}^{n} \mathcal{H}^{k}$-nulo e $\left(f_{i}\left(E_{i}\right)\right)_{i \in \mathbb{N}}$ sequência de imagens Lipschitz regulares induzidas por pares $\left(f_{i}, E_{i}\right)_{i \in \mathbb{N}}$, com $(\forall i) E_{i} \in \mathscr{B}_{\mathbb{R}^{k}}$ limitado, tais que

1. $M=M_{0} \cup \bigcup_{i \in \mathbb{N}} f_{i}\left(E_{i}\right)$;

2. para cada $i \in \mathbb{N}, \forall x, y \in E_{i}, \forall v \in \mathbb{R}^{k}$,

$$
\begin{aligned}
t^{-1}\|x-y\| & \leq\left\|f_{i}(x)-f_{i}(y)\right\| \leq t\|x-y\|, \\
t^{-1}\|v\| & \leq\left\|\mathrm{D} f_{i}(x) \cdot v\right\| \leq t\|v\|, \\
t^{-k} & \leq \mathrm{J} f_{i}(x) \leq t^{k}
\end{aligned}
$$

Demonstração. Pela proposição 4.3.iii), existem $\left(g_{i}\right)_{i \geq 1}$ sequência de aplicações Lipschitz $\mathbb{R}^{k} \rightarrow \mathbb{R}^{n}$ e $\left(A_{i}\right)_{i \geq 1}$ sequência de compactos em $\mathbb{R}^{k}$ tais que $M \backslash \cup_{i \geq 1} g_{i}\left(A_{i}\right)$ é $\mathcal{H}^{k}$-nulo.

Para cada $i \geq 1, g_{i}\left(A_{i}\right) \cap M$ é $\mathcal{H}^{k}$-mensurável (pois $g_{i}\left(A_{i}\right)$ é compacto, logo boreliano, e $M$ é $\mathcal{H}^{k}$-mensurável por hipótese) e tem medida $\mathcal{H}^{k}$ finita, pois $\mathcal{H}^{k}\left(g_{i}\left(A_{i}\right)\right) \leq\left(\operatorname{Lip} g_{i}\right)^{k} \mathcal{H}^{k}\left(A_{i}\right)=$ $\left(\operatorname{Lip} g_{i}\right)^{k} \mathcal{L}^{k}\left(A_{i}\right)<\infty$, em vista da proposição 2.49.iii), do teorema 2.54 e da compacidade de $A_{i}$. Portanto, como $\mathcal{H}^{k}$ é Borel-regular, segue do corolário 2.20 que existe $M_{i} \subset g_{i}\left(A_{i}\right) \cap M$ boreliano tal que $\mathcal{H}^{k}\left(g_{i}\left(A_{i}\right) \cap M \backslash M_{i}\right)=0$. Portanto,

$$
E_{i}:=g_{i}^{-1}\left(M_{i}\right) \cap A_{i} \cap J_{g_{i}}^{+}
$$

é um boreliano limitado de $\mathbb{R}^{k}$.

Defina $M_{0} \doteq M \backslash \cup_{i \geq 1} g_{i}\left(E_{i}\right)$, de modo que

$$
M=M_{0} \cup \bigcup_{i \geq 1} g_{i}\left(E_{i}\right)
$$

Afirmo que $\mathcal{H}^{k}\left(M_{0}\right)=0$. Com efeito, fixe $i \geq 1$. Tem-se $M_{i}=g_{i}\left(g_{i}^{-1}\left(M_{i}\right) \cap A_{i}\right), \log M_{i} \backslash g_{i}\left(E_{i}\right)=$ $g_{i}\left(g_{i}^{-1}\left(M_{i}\right) \cap A_{i}\right) \backslash g_{i}\left(E_{i}\right) \subset g_{i}\left(g_{i}^{-1}\left(M_{i}\right) \cap A_{i} \backslash E_{i}\right) \subset g_{i}\left(\mathbb{R}^{k} \backslash J_{g_{i}}^{+}\right)=g_{i}\left(\mathbb{R}^{k} \backslash D_{g_{i}}\right) \cup g_{i}\left(J_{g_{i}}^{0}\right)$. Mas,

- $\mathcal{H}^{k}\left(\mathbb{R}^{k} \backslash D_{g_{i}}\right)=\mathcal{L}^{k}\left(\mathbb{R}^{k} \backslash D_{g_{i}}\right)=0$, pelo teorema de Rademacher 2.97 e pelo teorema 2.54; logo, pela proposição 2.49,iii), $\mathcal{H}^{k}\left(g_{i}\left(\mathbb{R}^{k} \backslash D_{g_{i}}\right)\right) \leq\left(\operatorname{Lip} g_{i}\right)^{k} \mathcal{H}^{k}\left(\mathbb{R}^{k} \backslash D_{g_{i}}\right)=0$.

- $\chi_{g_{i}\left(J_{g_{i}}^{0}\right)} \leq \mathcal{H}^{0}\left(J_{g_{i}}^{0} \cap g_{i}^{-1}(\cdot)\right)$; logo, por monotonicidade da integral e pela fórmula da área:

$$
\begin{aligned}
\mathcal{H}^{k}\left(g_{i}\left(J_{g_{i}}^{0}\right)\right) & =\int \chi_{g_{i}\left(J_{g_{i}}^{0}\right)} \mathrm{d} \mathcal{H}^{k} \leq \int \mathcal{H}^{0}\left(J_{g_{i}}^{0} \cap g_{i}^{-1}\{y\}\right) \mathrm{d} \mathcal{H}^{k}(y) \stackrel{3.9}{=} \\
& =\int_{J_{g_{i}}^{0}} \mathrm{~J} g_{i} \mathrm{~d} \mathcal{L}^{k}=0 .
\end{aligned}
$$


Portanto, $M_{i} \backslash g_{i}\left(E_{i}\right)$ é $\mathcal{H}^{k}$-nulo. Como $i \geq 1$ foi tomado arbitrariamente e $M_{0} \subset(M \backslash$ $\left.\cup_{i \geq 1} g_{i}\left(A_{i}\right)\right) \cup\left(\cup_{i \geq 1}\left[g_{i}\left(A_{i}\right) \cap M \backslash M_{i}\right]\right) \cup\left(\cup_{i \geq 1} M_{i} \backslash g_{i}\left(E_{i}\right)\right)$, conclui-se que $M_{0}$ é $\mathcal{H}^{k}$-nulo, como afirmado.

Pelo teorema de linearização Lipschitz 3.4, para cada $i \geq 1$ existe uma sequência disjunta $\left(F_{j}^{i}\right)_{j \geq 1}$ em $\mathscr{B}_{\mathbb{R}^{k}}$ tal que $J_{g_{i}}^{+}=\dot{\cup}_{j \geq 1} F_{j}^{i}$ e, $\forall j \geq 1$, existe $S_{j}^{i} \in \operatorname{Sym}(k) \cap \operatorname{GL}\left(\mathbb{R}^{k}\right)$ tal que $\left(F_{j}^{i}, S_{j}^{i}\right)$ é uma $t$-linearização para $g_{i}$. Defina, para $i, j \geq 1, E_{j}^{i}:=E_{i} \cap F_{j}^{i}$; como $E_{i} \subset J_{g_{i}}^{+}$, segue $E_{i}=\dot{\cup}_{j \geq 1} E_{j}^{i}$, donde

$$
M=M_{0} \cup \bigcup_{i, j \geq 1} g_{i}\left(E_{j}^{i}\right) .
$$

Note que, para cada $i, j \geq 1, E_{j}^{i} \subset \mathbb{R}^{k}$ é boreliano (pois $E_{i}$ e $F_{j}^{i}$ o são) e limitado (pois $E_{j}^{i} \subset E_{i}$ e $E_{i}$ é limitado). Agora tome, para cada $i, j \geq 1, G_{j}^{i}:=S_{j}^{i}\left(E_{j}^{i}\right) \subset \mathbb{R}^{k}$, o qual é um boreliano limitado, e $f_{j}^{i}:=g_{i} \circ\left(S_{j}^{i}\right)^{-1}$. Então $f_{j}^{i}\left(G_{j}^{i}\right)=g_{i}\left(E_{j}^{i}\right)$, de modo que

$$
M=M_{0} \cup \bigcup_{i, j \geq 1} f_{j}^{i}\left(G_{j}^{i}\right)
$$

Além disso, para cada $i, j \geq 1,\left(G_{j}^{i}, \mathrm{id}_{\mathbb{R}^{k}}\right)$ é uma $t$-linearização para $f_{j}^{i}$, de forma que (4.2) é consequência de (ii) e (iii) na definição 3.1 e do corolário 3.3. Finalmente, $\mathcal{L}^{k}$-quase todo ponto de $G_{j}^{i}$ é tanto ponto de densidade 1 para $G_{j}^{i}$ como ponto de Lebesgue para $\mathrm{D} f_{j}^{i}$, em vista do corolário 2.70 e do teorema 2.74. Assim, subtraindo-se, um boreliano $\mathcal{L}^{k}$-nulo de $G_{j}^{i}$ (cuja imagem por $f_{j}^{i}$ é $\mathcal{H}^{k}$-nula e pode ser acrescentada a $\left.M_{0}\right)$, podemos supor que $\left(f_{j}^{i}, G_{j}^{i}\right)$ define uma imagem Lipschitz regular $f_{j}^{i}\left(G_{j}^{i}\right)$ no sentido da definição 4.5 .

A tese segue reenumerando-se $\left(f_{j}^{i}, G_{j}^{i}\right)_{i, j \geq 1}$ para $\left(f_{i}, G_{i}\right)_{i \geq 1}$.

Observação 4.8. Com a mesma hipótese e notação do teorema precedente, podemos ainda tomar $M_{0} \subset \mathbb{R}^{n}$ e $\left(f_{i}, E_{i}\right)_{i \in \mathbb{N}}$ satisfazendo as mesmas condições enunciadas na tese e a condição adicional de que a união enunciada no item 1 seja disjunta. Com efeito, tomando $M_{0} \subset \mathbb{R}^{n}$ e $\left(f_{i}, E_{i}\right)_{i \in \mathbb{N}}$ satisfazendo a tese, mostremos que os $E_{i}$ 's podem ser modificados de forma que a sequência $\left(f_{i}\left(E_{i}\right)\right)_{i \in \mathbb{N}}$ seja disjunta.

Para cada $i \geq 1, f_{i}\left(E_{i}\right)$ é $\mathcal{H}^{k}$-mensurável (pelo lema 3.7.i) e tem medida $\mathcal{H}^{k}$ finita (pois $E_{i}$ é limitado, e pela proposição 2.49.iii). Portanto, $\left\{f_{i}\left(E_{i}\right) \backslash \cup_{j<i} f_{j}\left(E_{j}\right)\right\}_{i \geq 1}$ é uma sequência disjunta de $\mathcal{H}^{k}$-mensuráveis em $M$ com medidas $\mathcal{H}^{k}$ finitas, cuja união é a mesma que a da sequência $\left(f_{i}\left(E_{i}\right)\right)_{i \geq 1}$. Pelo corolário 2.20, podemos, para cada $i \geq 1$, tomar $N_{i} \subset f_{i}\left(E_{i}\right) \backslash \cup_{j<i} f_{j}\left(E_{j}\right)$ boreliano tal que $\mathcal{H}^{k}\left(\left[f_{i}\left(E_{i}\right) \backslash \cup_{j<i} f_{j}\left(E_{j}\right)\right] \backslash N_{i}\right)=0, \operatorname{logo} M_{0}^{\prime}:=M \backslash \cup_{i \geq 1} N_{i}$ é $\mathcal{H}^{k}$-nulo e $\left(N_{i}\right)_{i \geq 1}$ é uma sequência disjunta de borelianos com cada $N_{i}$ contido na imagem de $\left.f_{i}\right|_{E_{i}}$. Substitua $M_{0}$ por $M_{0}^{\prime}$ e, para cada $i \geq 1$, substitua $E_{i}$ por $\left.f_{i}\right|_{E_{i}} ^{-1}\left(N_{i}\right)=f_{i}^{-1}\left(N_{i}\right) \cap E_{i} \in \mathscr{B}_{\mathbb{R}^{k}}$. Retirando do novo $E_{i}$ um conjunto Lebesgue-nulo, se necessário (cuja imagem por $f_{i}$ será $\mathcal{H}^{k}$-nula e pode ser acrescentada a $M_{0}$ ), ainda podemos supor que todo ponto do novo $E_{i}$ é um ponto de densidade 1 para $E_{i}$ e que, portanto, o par $\left(f_{i}, E_{i}\right)$ ainda define uma imagem Lipschitz regular. A nova sequência $\left(f_{i}\left(E_{i}\right)\right)_{i \geq 1}$ agora é disjunta e valem as mesmas condições anteriores.

\subsection{Propriedades Tangenciais dos Conjuntos Retificáveis}

Nesta seção, verificaremos que um subconjunto $k$-localmente retificável $\mathrm{M}$ em $\mathbb{R}^{n}$ admite em $\mathcal{H}^{k}$-quase todo ponto de $\mathrm{M}$ um espaço tangente aproximado (cf. teorema 4.10), o qual coincide com o espaço tangente usual da geometria diferencial caso $\mathrm{M}$ seja uma subvariedade diferenciável de dimensão $k$. A existência $\mathcal{H}^{n}$-q.s. de um tal espaço tangente aproximado permite abstrair, sob um ponto de vista da teoria da medida, noções e propriedades tangenciais usuais da geometria diferencial, a exemplo da noção de diferenciabilidade de aplicações definidas em M, como o faremos no próximo capítulo para o caso de aplicações Lipschitz; remetemos o leitor ao capítulo 3 de 
[9] para uma situação mais geral onde é discutida a noção de diferenciabilidade para aplicações aproximadamente diferenciáveis definidas em $\mathrm{M}$.

Notação. Dados $x \in \mathbb{R}^{n}$ e $r>0$, denotaremos por $\Phi_{x, r}: \mathbb{R}^{n} \rightarrow \mathbb{R}^{n}$ a aplicação definida por

$$
\Phi_{x, r}(y):=\frac{y-x}{r},
$$

de modo que $\Phi_{x, r}^{-1}(y)=x+r y$.

Recorde a notação e propriedades da noção de pushfoward de medidas introduzida no preâmbulo em 2.14.

Proposição 4.9. Sejam $0 \leq k \leq n, x \in \mathbb{R}^{n}$ e $r>0$. Então

$$
\left(\Phi_{x, r}\right)_{\#} \mathcal{H}^{k}=r^{k} \mathcal{H}^{k}
$$

Demonstração. Para todo $A \subset \mathbb{R}^{n}$,

$$
\mathcal{H}^{k}\left(\Phi_{x, r}^{-1}(A)\right)=\mathcal{H}^{k}(x+r A) \stackrel{2.49(i i)}{=} \mathcal{H}^{k}(r A)=r^{k} \mathcal{H}^{k}(A),
$$

sendo que a última igualdade decorre de 2.49(iii) aplicada à homotetia de razão $r, f: \mathbb{R}^{n} \rightarrow \mathbb{R}^{n}$, $x \mapsto r x$, è̀ sua inversa.

Para o próximo teorema, recorde a noção de convergência fraca-estrela de medidas de Radon, bem como a noção de densidade de medidas, introduzidas no preâmbulo em 2.87 e 2.66, respectivamente.

Teorema 4.10 (existência de espaços tangentes aproximados). Se $M \subset \mathbb{R}^{n}$ for um conjunto $k$ localmente retificável, então, para $\mathcal{H}^{k}$-q.t. $x \in M$, existe um único subespaço vetorial $k$-dimensional $\pi_{x} \subset \mathbb{R}^{n}$ tal que, para $r \downarrow 0$,

$$
\frac{\left(\Phi_{x, r}\right)_{\#}\left(\mathcal{H}^{k}\llcorner M)\right.}{r^{k}}=\mathcal{H}^{k}\left\llcorner( \frac { M - x } { r } ) \stackrel { * } { * } \mathcal { H } ^ { k } \left\llcorner\pi_{x} .\right.\right.
$$

Em particular, $\Theta^{k}\left(\mathcal{H}^{k}\llcorner M, x)=1\right.$ para $\mathcal{H}^{k}$-q.t. $x \in M$, i.e.

$$
\lim _{r \rightarrow 0^{+}} \frac{\mathcal{H}^{k}(M \cap \mathbb{B}(x, r))}{\alpha(k) r^{k}}=1
$$

para $\mathcal{H}^{k}$-q.t. $x \in M$.

Definição 4.11 (espaço tangente aproximado). Com a notação do teorema acima:

1) a família de medidas de Radon

$$
\frac{\left(\Phi_{x, r}\right)_{\#}\left(\mathcal{H}^{k}\llcorner M)\right.}{r^{k}}, \quad r>0
$$

diz-se obtida por blowup da medida de Radon $\mathcal{H}^{k}\llcorner M$.

2) para $x \in M$ tal que existe $\pi_{x}$, chamamos $\pi_{x}$ de espaço tangente aproximado a $M$ em $x$ e o denotamos por $\mathrm{T}_{x} M$.

Observação 4.12. Com a notação acima, a existência e o valor de $\mathrm{T}_{x} M$ dependem apenas da classe de equivalência de $M$ módulo conjuntos $\mathcal{H}^{k}$-nulos, pois a definição do referido subespaço só depende da medida de Radon $\mathcal{H}^{k}\llcorner M$. 

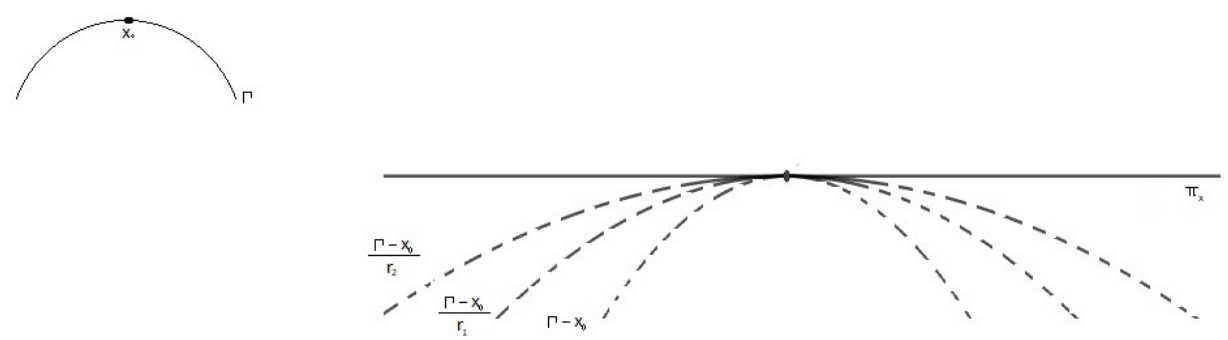

Figura 4.1: Os Blow-ups em $x_{0}$ da medida de Radon $\mathcal{H}^{1}\left\llcorner\Gamma\right.$ converge fraco estrela para $\mathcal{H}^{1}\llcorner\pi . N a$ imagem temos que $0<r_{2}<r_{1}<1$

Exemplo 4.13. Seja $\Gamma$ uma curva $C^{1}$ mergulhada em $\mathbb{R}^{n}$, i.e. $\Gamma=\gamma((a, b))$ onde $\gamma:(a, b) \rightarrow \mathbb{R}^{n}$ é um mergulho $C^{1}$. Dado $t_{0} \in(a, b)$, o espaço tangente a $\Gamma$ em $x_{0}=\gamma\left(t_{0}\right)$ é o subespaço unidimensional $\pi=\left\{s \gamma^{\prime}\left(t_{0}\right): s \in \mathbb{R}\right\} \subset \mathbb{R}^{n}$. Consideremos a família $\left(\mu_{x_{0}, r}\right)_{r>0}$ obtida por blowup da medida de Radon $\mu=\mathcal{H}^{1}\llcorner\Gamma$, cf. 4.11.1. Ou seja, para todo $r>0$,

$$
\mu_{x_{0}, r}=\frac{1}{r}\left(\Phi_{x, r}\right)_{\#}\left(\mathcal{H}^{1}\llcorner\Gamma)=\mathcal{H}^{1}\left\llcorner\left(\frac{\Gamma-x_{0}}{r}\right) .\right.\right.
$$

A ideia geométrica intuitiva de que, fazendo-se um "zoom" em torno de $x_{0}$, a curva $\Gamma$ se aproxima do referido espaço tangente $\pi$, pode ser justificada pelo fato de que, para $r \downarrow 0, \mu_{x_{0}, r}$ converge fracoestrela para a medida de Radon $\mathcal{H}^{1}\llcorner\pi$. De fato, isso é uma consequência do teorema 4.10 e da observação 4.16. Poder-se-ia tentar um argumento mais simples, como o que segue abaixo:

Para toda $\varphi \in \mathrm{C}_{\mathrm{c}}^{0}\left(\mathbb{R}^{n}\right)$, tem-se:

$$
\begin{aligned}
\int_{\mathbb{R}^{n}} \varphi \mathrm{d} \mu_{x_{0}, r} & =\frac{1}{r} \int_{\Gamma} \varphi\left(\frac{y-x_{0}}{r}\right) \mathrm{d} \mathcal{H}^{1}(y) \stackrel{\mathrm{FA}}{=} \frac{1}{r} \int_{a}^{b} \varphi\left(\frac{\gamma(t)-\gamma\left(t_{0}\right)}{r}\right)\left|\gamma^{\prime}(t)\right| \mathrm{d} t= \\
& =\int_{-\left(t_{0}-a\right) / r}^{\left(b-t_{0}\right) / r} \varphi\left(\frac{\gamma\left(t_{0}+r s\right)-\gamma\left(t_{0}\right)}{r}\right)\left|\gamma^{\prime}\left(t_{0}+r s\right)\right| \mathrm{d} s \stackrel{(?)}{\rightarrow} \int_{\mathbb{R}} \varphi\left(s \gamma^{\prime}\left(t_{0}\right)\right)\left|\gamma^{\prime}\left(t_{0}\right)\right| \mathrm{d} s \stackrel{\mathrm{FA}}{=} \\
& =\int_{\pi} \varphi \mathrm{d} \mathcal{H}^{1}=\int \varphi \mathrm{d} \mathcal{H}^{1}\llcorner\pi .
\end{aligned}
$$

No cálculo acima, na primeira aplicação da fórmula da área (FA) usou-se 3.12.ii) com a aplicação localmente Lipschitz $f=\gamma$ e na segunda aplicação da fórmula da área usou-se $3.10 \mathrm{com}$ a aplicação Lipschitz $\mathbb{R} \rightarrow \mathbb{R}^{n}$ dada por $s \mapsto s \gamma^{\prime}\left(t_{0}\right)$. O problema é justificar a convergência marcada com uma interrogação (?). A convergência indicada nesta passagem é verdadeira, pois o teorema e a observação mencionados acima garantem a convergência fraco-estrela $\mu_{x_{0}, r} \stackrel{*}{\rightarrow} \mathcal{H}^{1}\llcorner\pi$; no entanto, não vemos nenhuma maneira simples e direta de justificá-la. Caso $\gamma$ seja bi-Lipschitz sobre sua imagem, o argumento do passo 3 da prova do teorema 4.10 pode ser aplicado; no caso geral, só conseguimos justificar esta passagem através do teorema e da observação citados supra.

Na prova do teorema 4.10, usaremos o lema abaixo. Recorde a definição 2.76 no preâmbulo. Recorde também que $\mathrm{L}_{\text {loc }}^{1}\left(\mathcal{L}^{n}\right)$ admite naturalmente uma topologia de espaço de Fréchet induzida pelas seminormas $\left.\|\cdot\|_{L^{1}\left(\mathcal{L}^{n}\right.} L_{K}\right)$, com $K$ percorrendo os compactos não vazios de $\mathbb{R}^{n}$; para os leitores que não têm familiaridade com isso, basta adotar a seguinte:

Definição 4.14. Sejam $\left(f_{n}\right)_{n \in \mathbb{N}}$ uma sequência em $\mathrm{L}_{\text {loc }}^{1}\left(\mathcal{L}^{n}\right)$ e $f \in \mathrm{L}_{\text {loc }}^{1}\left(\mathcal{L}^{n}\right)$. Diz-se que $f_{n} \rightarrow f$ em $\mathrm{L}_{\text {loc }}^{1}\left(\mathcal{L}^{n}\right)$ se, para todo $K \subset \mathbb{R}^{n}$ compacto, $\left.\left.f_{n}\right|_{K} \rightarrow f\right|_{K}$ em $\mathrm{L}^{1}(K)$. Definição análoga se aplica para um net de funções no lugar de uma sequência.

Lema 4.15. Sejam $f \in \mathrm{L}_{\text {loc }}^{1}\left(\mathcal{L}^{n}\right)$ e $y \in \mathbb{R}^{n}$ ponto de Lebesgue de $f$. Defina, para $r>0, g_{r}: \mathbb{R}^{n} \rightarrow \mathbb{R}$ por $g_{r}(w):=f(y+r w)$. Então $g_{r} \in \mathrm{L}_{\text {loc }}^{1}\left(\mathcal{L}^{n}\right)$ e $g_{r} \stackrel{r \rightarrow 0}{\rightarrow} f(y)$ em $\mathrm{L}_{\text {loc }}^{1}\left(\mathcal{L}^{n}\right)$, i.e. converge para a função constante e igual a $f(y)$ em $\mathrm{L}_{\text {loc }}^{1}\left(\mathcal{L}^{n}\right)$. 
Demonstração. O fato de que $g_{r} \in \mathrm{L}_{\text {loc }}^{1}\left(\mathcal{L}^{n}\right)$ para $r>0$ decorre imediatamente do teorema de mudança de variáveis. Além disso, pela hipótese de ser $y$ ponto de Lebesgue de $f$, tem-se:

$$
\lim _{r \rightarrow 0} \frac{1}{\alpha(n) r^{n}} \int_{\mathbb{B}(y, r)}|f(x)-f(y)| \mathrm{d} x=0 .
$$

Mas, pelo teorema de mudança de variáveis, para todo $R>0$,

$$
\begin{gathered}
\frac{1}{\alpha(n) r^{n}} \int_{\mathbb{B}(y, r)}|f(x)-f(y)| \mathrm{d} x \\
\stackrel{x=y+r w}{=} \frac{1}{\alpha(n)} \int_{\mathbb{B}(0,1)}|f(y+r w)-f(y)| \mathrm{d} w \stackrel{w=u / R}{=} \\
=\frac{1}{\alpha(n) R^{n}} \int_{\mathbb{B}(0, R)}\left|f\left(y+\frac{r}{R} u\right)-f(y)\right| \mathrm{d} u,
\end{gathered}
$$

portanto

$$
\lim _{r \rightarrow 0} \int_{\mathbb{B}(0, R)}\left|f\left(y+\frac{r}{R} u\right)-f(y)\right| \mathrm{d} u=0 .
$$

Daí, para todo $R>0$, tomando $\left(r_{i}\right)_{i \in \mathbb{N}}$ sequência em $(0, \infty)$ com $r_{i} \downarrow 0$, tem-se

$$
\begin{aligned}
\left\|g_{r_{i}}-f(y)\right\|_{\mathrm{L}^{1}(\mathbb{B}(0, R))} & =\int_{\mathbb{B}(0, R)}\left|f\left(y+r_{i} w\right)-f(y)\right| \mathrm{d} w= \\
& =\int_{\mathbb{B}(0, R)}\left|f\left(y+(\underbrace{r_{i} R}_{i \rightarrow \infty}) \frac{w}{R}\right)-f(y)\right| \mathrm{d} w \stackrel{i \rightarrow \infty}{\rightarrow} 0 .
\end{aligned}
$$

Prova do teorema 4.10. 1) Unicidade. Suponha que, dado $x \in M$, existam subespaços vetoriais $k$ dimensionais $\pi_{x}$ e $\eta_{x}$ de $\mathbb{R}^{n}$ tais que $\mathcal{H}^{k}\left\llcorner\left(\frac{M-x}{r}\right) \stackrel{*}{\rightarrow} \mathcal{H}^{k}\left\llcorner\pi_{x}\right.\right.$ e $\mathcal{H}^{k}\left\llcorner\left(\frac{M-x}{r}\right) \stackrel{*}{\rightarrow} \mathcal{H}^{k}\left\llcorner\eta_{x}\right.\right.$. Então $\mathcal{H}^{k}\left\llcorner\pi_{x}=\mathcal{H}^{k}\left\llcorner\eta_{x}\right.\right.$, o que implica $\pi_{x}=\eta_{x}$.

2) Provemos a existência. Devemos mostrar que, para $\mathcal{H}^{k}$-q.t. $x \in M$, existe $\pi_{x}$ subespaço $k$ dimensional de $\mathbb{R}^{n}$ tal que, para toda $\varphi \in \mathrm{C}_{\mathrm{c}}\left(\mathbb{R}^{n}\right)$

$$
\lim _{r \rightarrow 0} \frac{1}{r^{k}} \int_{M} \varphi \circ \Phi_{x, r} \mathrm{~d} \mathcal{H}^{k}=\int_{\pi_{x}} \varphi \mathrm{d} \mathcal{H}^{k} .
$$

3) Caso 1: suponha que $M=f(E)$ é imagem Lipschitz regular definida por $(f, E)$, com $f: \mathbb{R}^{k} \rightarrow \mathbb{R}^{n}$ Lipschitz, $E \subset \mathbb{R}^{k}$ boreliano limitado e $\left.f\right|_{E} ^{-1}$ Lipschitz. Provemos que, para todo $x=f(z)$ em $M, \mathrm{~T}_{x} M=\mathrm{D} f(z) \cdot \mathbb{R}^{k}$. Com efeito, dada $\varphi \in \mathrm{C}_{\mathrm{c}}\left(\mathbb{R}^{n}\right)$, para todo $r>0$, tem-se:

$$
\begin{aligned}
\frac{1}{r^{k}} \int_{M} \varphi \circ \Phi_{x, r} \mathrm{~d} \mathcal{H}^{k} & =\frac{1}{r^{k}} \int_{M} \varphi\left(\frac{y-x}{r}\right) \mathrm{d} \mathcal{H}^{k}(y) \stackrel{\text { f. } \stackrel{\text { área }}{=}}{ } \\
& =\frac{1}{r^{k}} \int_{E} \varphi\left(\frac{f(w)-f(z)}{r}\right) \mathrm{J} f(w) \mathrm{d} \mathcal{L}^{k}(w)= \\
& =\int_{\mathbb{R}^{k}} u_{r}(w) \mathrm{d} w,
\end{aligned}
$$

onde $u_{r}: \mathbb{R}^{k} \rightarrow \mathbb{R}$ é dada por

$$
u_{r}(w):=\chi_{E}(z+r w) \varphi\left(\frac{f(z+r w)-f(z)}{r}\right) \mathrm{J} f(z+r w) .
$$

Afirmação 1: $u_{r} \rightarrow u_{0}$ em $L_{\text {loc }}^{1}\left(\mathcal{L}^{k}\right)$, onde $u_{0}: \mathbb{R}^{k} \rightarrow \mathbb{R}$ é dada por $u_{0}(w):=\varphi(\operatorname{D} f(z) \cdot w) \mathrm{J} f(z)$. 
Com efeito, para todo $R>0$,

$$
\begin{aligned}
& \left\|u_{r}-u_{0}\right\|_{\mathrm{L}^{1}(\mathbb{B}(0, R))} \stackrel{\text { des. triangular }}{\leq} \\
& \leq \int_{\mathbb{B}(0, R)} \underbrace{\mid \chi_{E}(z+r w)-\overbrace{\chi_{E}(z) \mid}^{=1}}_{\substack{r \rightarrow 0 \\
\rightarrow 0 \mathrm{em} \mathrm{L} \mathrm{L}^{1}(\mathbb{B}(0, R)) \text { pelo lema }} .15} \underbrace{\varphi\left(\frac{f(z+r w)-f(z)}{r}\right) \mid}_{\leq\|\varphi\|_{u}} \underbrace{|\mathrm{J} f(z+r w)|}_{\leq(\operatorname{Lip} f)^{k}} \mathrm{~d} w+ \\
& +\int_{\mathbb{B}(0, R)} \underbrace{\varphi\left(\frac{f(z+r w)-f(z)}{r}\right)-\varphi(\mathrm{D} f(z) \cdot w) \mid}_{\leq 2\|\varphi\|_{u} \mathrm{e}^{r \rightarrow 0} 0 \text { pontualmente }} \underbrace{|\mathrm{J} f(z+r w)|}_{\leq(\operatorname{Lip} f)^{k}} \mathrm{~d} w+ \\
& +\int_{\mathbb{B}(0, R)} \underbrace{|\varphi(\mathrm{D} f(z) \cdot w)|}_{\leq\|\varphi\|_{u}} \underbrace{|\mathrm{J} f(z+r w)-\mathrm{J} f(z)|}_{r \rightarrow 00 \mathrm{em} \mathrm{L}^{1}(\mathbb{B}(0, R)) \text { por } 4.6,4.15} \mathrm{~d} w,
\end{aligned}
$$

o que prova a afirmação 1 , pois o segundo membro converge para zero se $r \rightarrow 0$ (na segunda integral aplicamos o teorema da convergência dominada).

Afirmação 2: existe $R>0$ tal que, para todo $r>0$, spt $u_{r} \subset \mathbb{B}(0, R)$. De fato, tomando $\lambda>0$ tal que $\lambda^{-1}$ seja constante de Lipschitz para $\left.f\right|_{E} ^{-1}$, tem-se, $\forall z^{\prime} \in E$,

$$
\left|f\left(z^{\prime}\right)-f(z)\right| \geq \lambda\left|z^{\prime}-z\right| .
$$

Assim, se $\rho>0$ é tal que spt $\varphi \subset \mathbb{B}(0, \rho)$, tem-se, para todo $r>0$ e todo $w \in \mathbb{R}^{k}$ tal que $u_{r}(w) \neq 0$ :

- $\chi_{E}(z+r w) \neq 0, \operatorname{logo} z^{\prime}=z+r w \in E$, donde $\lambda r|w| \leq|f(z+r w)-f(z)|$;

- $\varphi\left(\frac{f(z+r w)-f(z)}{r}\right) \neq 0, \log 0|f(z+r w)-f(z)| \leq r \rho$.

Portanto,

$$
r \rho \geq|f(z+r w)-f(z)| \geq \lambda r|w|,
$$

donde $|w| \leq R:=\rho / \lambda$. Ou seja, para todo $r>0$, spt $u_{r}=\overline{\left\{u_{r} \neq 0\right\}} \subset \mathbb{B}(0, R)$, o que prova a afirmação 2 .

Combinando-se as afirmações 1 e 2 , conclui-se que $u_{r} \rightarrow u_{0}$ em $\mathrm{L}^{1}\left(\mathcal{L}^{k}\right)$. Portanto, em vista de (4.6), obtém-se

$$
\begin{aligned}
\frac{1}{r^{k}} \int_{M} \varphi \circ \Phi_{x, r} \mathrm{~d} \mathcal{H}^{k} & \stackrel{r \rightarrow 0}{\rightarrow} \int_{\mathbb{R}^{k}} u_{0} \mathrm{~d} \mathcal{L}^{k}= \\
& =\int_{\mathbb{R}^{k}} \varphi(\mathrm{D} f(z) \cdot w) \mathrm{J} f(z) \mathrm{d} w \stackrel{\text { f. área }}{=} \\
& =\int_{\mathrm{D} f(z) \cdot \mathbb{R}^{k}} \varphi \mathrm{d} \mathcal{H}^{k} .
\end{aligned}
$$

Está provado, pois, o caso $1, \operatorname{com} \pi_{x}=\mathrm{D} f(z) \cdot \mathbb{R}^{k}$ para todo $x=f(z) \in f(E)=M$.

4) Caso geral. Pelo teorema de decomposição 4.7, existem $M_{0} \subset \mathbb{R}^{n} \mathcal{H}^{k}$-nulo e $\left(f_{i}\left(E_{i}\right)\right)_{i \in \mathbb{N}}$ sequência de imagens Lipschitz regulares induzidas por pares $\left(f_{i}, E_{i}\right)_{i \in \mathbb{N}} \operatorname{com}(\forall i \in \mathbb{N}) E_{i} \in \mathscr{B}_{\mathbb{R}^{k}}$ limitado, $\left.f_{i}\right|_{E_{i}} ^{-1}$ Lipschitz e

$$
M=M_{0} \cup \bigcup_{i \in \mathbb{N}} f_{i}\left(E_{i}\right)
$$


Pelo caso 1 , para todo $i \in \mathbb{N}$, todo $x \in M_{i}:=f_{i}\left(E_{i}\right)$ e toda $\varphi \in \mathrm{C}_{\mathrm{c}}\left(\mathbb{R}^{n}\right)$, tem-se

$$
\lim _{r \rightarrow 0} \frac{1}{r^{k}} \int_{M_{i}} \varphi \circ \Phi_{x, r} \mathrm{~d} \mathcal{H}^{k}=\int_{\pi_{x}} \varphi \mathrm{d} \mathcal{H}^{k},
$$

onde $\pi_{x}=\mathrm{D} f_{i}\left(\left.f_{i}\right|_{E_{i}} ^{-1}(x)\right) \cdot \mathbb{R}^{k}$.

Note que, para todo $i \in \mathbb{N}, M_{i}=f_{i}\left(E_{i}\right) \subset M$ é $\mathcal{H}^{k}$-mensurável (logo $\mathcal{H}^{k}\llcorner M$-mensurável), pelo lema 3.7.i), e $\mathcal{H}^{k}\left\llcorner M\right.$ é uma medida de Radon em $\mathbb{R}^{n}$ (pois $M$ é $k$-localmente retificável por hipótese), logo Borel-regular e aberta $\sigma$-finita. Podemos, pois, aplicar o teorema da densidade superior $2.69 \operatorname{com} \mu=\mathcal{H}^{k}\left\llcorner M\right.$ e $B=\mathbb{R}^{n} \backslash \mathrm{M}_{i}$ para concluir que, para $\mathcal{H}^{k}$-q.t. $x \in M_{i}$,

$$
\Theta^{k}\left(\mathcal{H}^{k}\left\llcorner M, \mathbb{R}^{n} \backslash M_{i}, x\right)=\Theta^{* k}\left(\mathcal{H}^{k}\left\llcorner M, \mathbb{R}^{n} \backslash M_{i}, x\right)=0 .\right.\right.
$$

Por outro lado, para todo $i \in \mathbb{N}, r>0, x \in \mathbb{R}^{n}$ e $\varphi \in \mathrm{C}_{\mathrm{c}}\left(\mathbb{R}^{n}\right)$, tomando-se $R>0$ tal que spt $\varphi \subset \mathbb{B}(0, R)$, tem-se

$$
\begin{aligned}
\left|\frac{1}{r^{k}} \int_{M \backslash M_{i}} \varphi \circ \Phi_{x, r} \mathrm{~d} \mathcal{H}^{k}\right| & =\frac{1}{r^{k}}\left|\int_{M \backslash M_{i}} \varphi\left(\frac{y-x}{r}\right) \mathrm{d} \mathcal{H}^{k}(y)\right| \leq \\
& \leq \frac{1}{r^{k}} \int_{\mathbb{B}(x, r R) \cap M \backslash M_{i}}\left|\varphi\left(\frac{y-x}{r}\right)\right| \mathrm{d} \mathcal{H}^{k}(y) \leq \\
& \leq\|\varphi\|_{u} R^{k} \alpha(k) \frac{1}{\alpha(k)(r R)^{k}} \int_{\mathbb{B}(x, r R)} \chi_{M \backslash M_{i}}(y) \mathrm{d} \mathcal{H}^{k}(y)= \\
& =\|\varphi\|_{u} R^{k} \alpha(k) \frac{\mathcal{H}^{k}\left\llcorner M \backslash M_{i}(\mathbb{B}(x, r R))\right.}{\alpha(k)(r R)^{k}} .
\end{aligned}
$$

Portanto, para todo $i \in \mathbb{N}, x \in \mathbb{R}^{n}$ e $\varphi \in \mathrm{C}_{\mathrm{c}}\left(\mathbb{R}^{n}\right)$,

$$
\limsup _{r \rightarrow 0}\left|\frac{1}{r^{k}} \int_{M \backslash M_{i}} \varphi \circ \Phi_{x, r} \mathrm{~d} \mathcal{H}^{k}\right| \leq\|\varphi\|_{u} R^{k} \alpha(k) \Theta^{* k}\left(\mathcal{H}^{k}\left\llcorner M, \mathbb{R}^{n} \backslash M_{i}, x\right) .\right.
$$

De (4.8) e (4.9) conclui-se que, para todo $i \in \mathbb{N}, \varphi \in \mathrm{C}_{\mathrm{c}}\left(\mathbb{R}^{n}\right)$ e para $\mathcal{H}^{k}$-q.t. $x \in M_{i}$,

$$
\lim _{r \rightarrow 0} \frac{1}{r^{k}} \int_{M \backslash M_{i}} \varphi \circ \Phi_{x, r} \mathrm{~d} \mathcal{H}^{k}=0 .
$$

Daí, de (4.7) e de (4.10), segue, para todo $i \in \mathbb{N}, \varphi \in \mathrm{C}_{\mathrm{c}}\left(\mathbb{R}^{n}\right)$ e para $x \in M_{i} \backslash Z_{i} \operatorname{com} Z_{i} \subset M_{i}$ $\mathcal{H}^{k}$-nulo,

$$
\lim _{r \rightarrow 0} \frac{1}{r^{k}} \int_{M} \varphi \circ \Phi_{x, r} \mathrm{~d} \mathcal{H}^{k}=\int_{\pi_{x}} \varphi \mathrm{d} \mathcal{H}^{k},
$$

onde $\pi_{x}=\mathrm{D} f_{i}\left(\left.f_{i}\right|_{E_{i}} ^{-1}(x)\right) \cdot \mathbb{R}^{k}$. Isso conclui a prova do caso geral, i.e. mostramos que o espaço tangente aproximado $\mathrm{T}_{x} M$ existe no complementar em $M$ do $\mathcal{H}^{k}$-nulo $M_{0} \cup \bigcup_{i \in \mathbb{N}} Z_{i}$.

5) Sejam $\mathbb{B}^{n}=\mathbb{B}(0,1) \subset \mathbb{R}^{n}$ e $x \in M$ tal que vale (4.3). Como $\pi_{x} \cap \partial \mathbb{B}^{n}$ é uma esfera de dimensão $k-1$ (a qual tem dimensão de Hausdorff $k-1$, em vista do exemplo 4.4), segue $\mathcal{H}^{k}\left\llcorner\pi_{x}\left(\partial \mathbb{B}^{n}\right)=0\right.$. Daí, de (4.3) e do teorema 2.89, obtém-se

$$
\begin{aligned}
\alpha(k) & =\mathcal{H}^{k}\left\llcorner\pi_{x}\left(\mathbb{B}^{n}\right)=\right. \\
& =\lim _{r \rightarrow 0} \frac{\mathcal{H}^{k}\left\llcorner M\left(\Phi_{x, r}^{-1} \mathbb{B}^{n}\right)\right.}{r^{k}}= \\
& =\lim _{r \rightarrow 0} \frac{\mathcal{H}^{k}(M \cap \mathbb{B}(x, r))}{r^{k}} .
\end{aligned}
$$


Portando, $\Theta^{k}\left(\mathcal{H}^{k}\llcorner M, x)=1\right.$ para todo $x \in M$ que satisfaça (4.3); em particular, isso vale para $\mathcal{H}^{k}$-q.t. $x \in M$.

Observação 4.16. Com a notação da demonstração do teorema 4.10, se $M_{i}=f_{i}\left(E_{i}\right)$ for uma vizinhança de $x$ em $M$ (i.e. vizinhança na topologia relativa), então $\Theta^{k}\left(\mathcal{H}^{k}\left\llcorner M, \mathbb{R}^{n} \backslash M_{i}, x\right)=0\right.$. Daí, segue do argumento usado no passo 4 (caso geral) que o espaço tangente aproximado em $x$ existe e é dado por $\mathrm{T}_{x} M=\mathrm{D} f_{i}\left(\left.f_{i}\right|_{E_{i}} ^{-1}(x)\right) \cdot \mathbb{R}^{k}$. Em particular, se $M$ for uma subvariedade $\mathrm{C}^{1}$ mergulhada em $\mathbb{R}^{n}, M$ admite espaço tangente aproximado em todo $x \in M$, o qual coincide com o espaço tangente usual do Cálculo em Variedades.

Observação 4.17. Na demonstração do teorema 4.10, provamos no passo 5) que (4.4) vale para todo $x \in M$ tal que (4.3) é verificada.

Proposição 4.18. Sejam $M_{1}, M_{2} \subset \mathbb{R}^{n}$ k-localmente retificáveis. Então $M_{1} \cup M_{2}$ é $k$-localmente retificável.

Demonstração. Note que $M_{1} \cup M_{2}$ é $k$-enumeravelmente retificável (em vista da proposição 4.3.ii), $\operatorname{logo} \mu=\mathcal{H}^{k}\left\llcorner M_{1} \cup M_{2}\right.$ é Borel-regular (em vista da proposição 4.3.v) e localmente finita, pois $\mathcal{H}^{k}\left\llcorner M_{1} \cup M_{2} \leq \mathcal{H}^{k}\left\llcorner M_{1}+\mathcal{H}^{k}\left\llcorner M_{1}\right.\right.\right.$ e as duas medidas no segundo membro são de Radon pela hipótese. Portanto, a tese segue de 4.3.vi).

Notação. Dados $x \in \mathbb{R}^{n}, r>0$ e $M \subset \mathbb{R}^{n}$, denotamos por $M_{x, r}$ a imagem de $M$ por $\Phi_{x, r}$, i.e. $\frac{M-x}{r}$.

Proposição 4.19 (a noção de espaço tangente aproximado é local). Sejam $M_{1}, M_{2} \subset \mathbb{R}^{n} k$ localmente retificáveis. Então, para $\mathcal{H}^{k}$-q.t. $x \in M_{1} \cap M_{2}$,

$$
\mathrm{T}_{x} M_{1}=\mathrm{T}_{x} M_{2} .
$$

Demonstração. Para $\mathcal{H}^{k}$-q.t. $x \in M_{1} \cap M_{2}$, tem-se

$$
\begin{aligned}
\mathcal{H}^{k} & \left(M_{1} \triangle M_{2}\right)_{x, r}(\mathbb{B}(0,1)) \stackrel{4.9}{=} \frac{\left(\Phi_{x, r}\right)_{\#}\left(\mathcal{H}^{k}\left\llcorner M_{1} \triangle M_{2}\right)\right.}{r^{k}}(\mathbb{B}(0,1))= \\
= & \frac{\mathcal{H}^{k}\left(\left(M_{1} \triangle M_{2}\right) \cap \mathbb{B}(x, r)\right)}{r^{k}} \stackrel{r \rightarrow 0}{\rightarrow} 0,
\end{aligned}
$$

em vista do teorema da densidade superior $2.69 \operatorname{com} \mu=\mathcal{H}^{k}\left\llcorner M_{1} \cup M_{2}\right.$ (a qual é de Radon em $\mathbb{R}^{n}$, em vista da proposição 4.18, logo é Borel-regular e aberta $\sigma$-finita) e $B=M_{1} \triangle M_{2}$.

Dado $K \subset \mathbb{R}^{n}$ compacto, existe $R>0$ tal que $K \subset \mathbb{B}(0, R)=R \cdot \mathbb{B}^{n}$. Daí, de (4.11) segue que, para $\mathcal{H}^{k}$-q.t. $x \in M_{1} \cap M_{2}$,

$$
\begin{aligned}
\mathcal{H}^{k}\left(K \cap\left(M_{1} \triangle M_{2}\right)_{x, r}\right) & \leq \mathcal{H}^{k}\left(R \cdot \mathbb{B}^{n} \cap R \cdot R^{-1} \cdot\left[M_{1} \triangle M_{2}\right]_{x, r}\right)= \\
& =R^{k} \mathcal{H}^{k}(\mathbb{B}^{n} \cap\left[M_{1} \triangle M_{2}\right]_{x} \underbrace{r R}_{r \rightarrow 0}) \underset{(4.11)}{\stackrel{r \rightarrow 0}{\rightarrow} 0 .} .
\end{aligned}
$$

Por outro lado, para todo $x \in \mathbb{R}^{n}, r>0$ e $\varphi \in \mathrm{C}_{\mathrm{c}}\left(\mathbb{R}^{n}\right)$, tem-se, para $i=1,2$ :

$$
\begin{aligned}
\frac{1}{r^{k}} \int_{M_{i}} \varphi \circ \Phi_{x, r} \mathrm{~d} \mathcal{H}^{k} & =\frac{1}{r^{k}} \int \varphi \mathrm{d}\left[\left(\Phi_{x, r}\right)_{\#}\left(\mathcal{H}^{k}\left\llcorner M_{i}\right)\right]=\right. \\
& =\int \varphi \mathrm{d}\left[\mathcal{H}^{k}\left\llcorner\left(M_{i}\right)_{x, r}\right]=\right. \\
& =\int_{\left(M_{i}\right)_{x, r}} \varphi \mathrm{d} \mathcal{H}^{k} .
\end{aligned}
$$


Daí,

$$
\begin{aligned}
& \left|\frac{1}{r^{k}} \int_{M_{1}} \varphi \circ \Phi_{x, r} \mathrm{~d} \mathcal{H}^{k}-\frac{1}{r^{k}} \int_{M_{2}} \varphi \circ \Phi_{x, r} \mathrm{~d} \mathcal{H}^{k}\right|= \\
& =\left|\int_{\left(M_{1}\right)_{x, r}} \varphi \mathrm{d} \mathcal{H}^{k}-\int_{\left(M_{2}\right)_{x, r}} \varphi \mathrm{d} \mathcal{H}^{k}\right| \leq \\
& \leq \int\left|\chi_{\left(M_{1}\right)_{x, r}}-\chi_{\left(M_{2}\right)_{x, r}}\right||\varphi| \mathrm{d} \mathcal{H}^{k}= \\
& =\int_{\left(M_{1}\right)_{x, r} \triangle\left(M_{2}\right)_{x, r}}|\varphi| \mathrm{d} \mathcal{H}^{k} \leq \\
& \leq\|\varphi\|_{u} \mathcal{H}^{k}\left(\operatorname{spt} \varphi \cap\left(M_{1} \triangle M_{2}\right)_{x, r}\right) .
\end{aligned}
$$

Portanto, como spt $\varphi$ é compacto, para $x \in M_{1} \cap M_{2}$ tal que valha (4.12) e existam ambos os espaços tangentes aproximados $\mathrm{T}_{x} M_{1}$ e $\mathrm{T}_{x} M_{2}$ (ou seja, para $\mathcal{H}^{k}$-q.t. $x \in M_{1} \cap M_{2}$ ), tomandose $r \rightarrow 0$ acima conclui-se que $\int \varphi \mathrm{d}\left(\mathcal{H}^{k}\left\llcorner\mathrm{~T}_{x} M_{1}\right)=\int \varphi \mathrm{d}\left(\mathcal{H}^{k}\left\llcorner\mathrm{~T}_{x} M_{2}\right)\right.\right.$. Pela arbitrariedade da $\varphi \in \mathrm{C}_{\mathrm{c}}\left(\mathbb{R}^{n}\right)$ tomada, segue que as medidas de Radon $\mathcal{H}^{k}\left\llcorner\mathrm{~T}_{x} M_{1}\right.$ e $\mathcal{H}^{k}\left\llcorner\mathrm{~T}_{x} M_{2}\right.$ coincidem. Logo, $\mathrm{T}_{x} M_{1}=\mathrm{T}_{x} M_{2}$.

\subsection{Retificabilidade a partir de blow-ups de medidas de Radon}

Nesta seção será enunciada e provada uma recíproca parcial do teorema 4.10. Trata-se do teorema 4.21, que fornece um critério para retificabilidade a partir da existência de espaços tangentes aproximados.

Notação. Seja $\pi$ um subespaço $k$-dimensional de $\mathbb{R}^{n}$. Denotaremos por $\operatorname{pr}_{\pi}$ e $\operatorname{pr}_{\pi}^{\perp}$ as projeções ortogonais em $\pi$ e $\pi^{\perp}$, respectivamente. Para cada $t>0$, definimos o cone fechado

$$
K(\pi, t):=\left\{y \in \mathbb{R}^{n} \mid\left\|\mathrm{pr}_{\pi}^{\perp} \cdot y\right\| \leq t\left\|\mathrm{pr}_{\pi} \cdot y\right\|\right\} .
$$

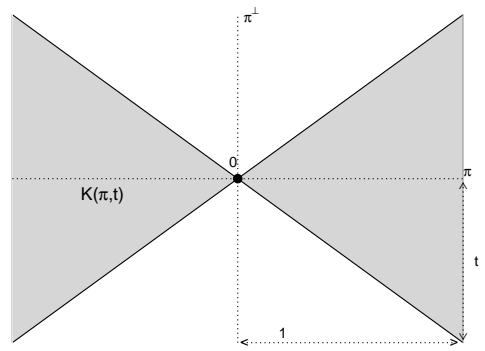

Figura 4.2: Cone fechado

Lema 4.20 (critério para retificabilidade). Sejam $\mathrm{M} \subset \mathbb{R}^{n}$ limitado e $\pi \subset \mathbb{R}^{n}$ um subespaço vetorial de dimensão $k$. Suponha que existam $\delta, t>0$ tais que, para todo $x \in \mathrm{M}$,

$$
\mathrm{M} \cap \mathbb{B}(x, \delta) \subset x+K(\pi, t) .
$$

Então existem famílias finitas $\left(f_{i}\right)_{1 \leq i \leq N}$ de aplicações Lipschitz $\mathbb{R}^{k} \rightarrow \mathbb{R}^{n}$ e $\left(F_{i}\right)_{1 \leq i \leq N}$ de subconjuntos limitados em $\mathbb{R}^{k}$ tais que

$$
\mathrm{M}=\bigcup_{i=1}^{N} f_{i}\left(F_{i}\right) .
$$

Em particular, se $\mathrm{M}$ for $\mathcal{H}^{k}$-mensurável, então $\mathrm{M}$ é k-retificável.

Demonstração. Se $x_{0} \in \mathrm{M}$ e $x, y \in \mathbb{B}\left(x_{0}, \delta / 2\right)$, então $y \in \mathbb{B}(x, \delta) \cap \mathrm{M} \subset x+K(\pi, t)$, i.e. $\| \operatorname{pr}_{\pi}^{\perp}(y-$ $x)\|\leq t\| \operatorname{pr}_{\pi}(y-x) \|$. Em particular, $\operatorname{pr}_{\pi}$ é uma bijeção de $\mathrm{M} \cap \mathbb{B}\left(x_{0}, \delta / 2\right)$ sobre o conjunto limitado 
$G_{x_{0}}:=\operatorname{pr}_{\pi}\left(\mathrm{M} \cap \mathbb{B}\left(x_{0}, \delta / 2\right)\right) \subset \pi$. Assim, a cada $x_{0} \in \mathrm{M}$ associamos o subconjunto limitado $G_{x_{0}}$ de $\pi \equiv \mathbb{R}^{k}$ e a aplicação $g_{x_{0}}: G_{x_{0}} \rightarrow \mathbb{R}^{n}$ que inverte $\left.\operatorname{pr}_{\pi}\right|_{M \cap \mathbb{B}\left(x_{0}, \delta / 2\right)}$, de modo que $g_{x_{0}}$ é Lipschitz com $\operatorname{Lip}\left(g_{x_{0}}\right) \leq \sqrt{1+t^{2}}$.

Como $\mathrm{M}$ é um subespaço métrico de $\mathbb{R}^{n}$ totalmente limitado (pois todo subconjunto limitado de $\mathbb{R}^{n}$ é totalmente limitado), podemos cobri-lo com um número finito de bolas de raios $\delta / 2$, de modo que existem $N \in \mathbb{N}$ e $x_{1}, \ldots, x_{N} \in \mathrm{M}$ tais que

$$
\mathrm{M}=\bigcup_{i=1}^{N} \mathrm{M} \cap \mathbb{B}\left(x_{i}, \delta / 2\right)=\bigcup_{i=1}^{N} g_{x_{i}}\left(G_{x_{i}}\right) .
$$

A primeira afirmação segue, pois, compondo-se cada $g_{x_{i}}, 1 \leq i \leq N$, com uma injeção ortogonal $P: \mathbb{R}^{k} \rightarrow \mathbb{R}^{n}$ tal que $P\left(\mathbb{R}^{k}\right)=\pi$ e aplicando-se o teorema de Kirszbraun 2.95.

Para provar a segunda afirmação, note que, se $\mathrm{M}$ for $\mathcal{H}^{k}$-mensurável, então $\mathrm{M}$ é $k$-enumeravelmente retificável pela proposição 4.3.iii). Além disso, $\mathcal{H}^{k}(\mathrm{M}) \leq \sum_{i=1}^{N} \mathcal{H}^{k}\left(f_{i}\left(F_{i}\right)\right) \leq \sum_{i=1}^{n}\left(\operatorname{Lip} f_{i}\right)^{k} \mathcal{L}^{k}\left(F_{i}\right)<$ $\infty$, por 2.49.iii), 2.54 e pelo fato de os $F_{i}$ 's serem limitados. Sendo $M$ um conjunto $\mathcal{H}^{k}$-mensurável de medida $\mathcal{H}^{k}$-finita e sendo $\mathcal{H}^{k}$ uma medida Borel-regular, segue da proposição 2.26.i) que $\mathcal{H}^{k}\llcorner M$ é uma medida Borel-regular finita, logo uma medida de Radon finita, em vista da observação 2.24.2). Portanto, M é $k$-retificável, como afirmado.

Teorema 4.21 (Retificabilidade a partir da convergência de blow-ups). Sejam $\mu$ uma medida de Radon em $\mathbb{R}^{n}$ e $\mathrm{M} \subset \mathbb{R}^{n}$ boreliano tais que $\mu$ é concentrada em $\mathrm{M}$ (i.e. $\mu=\mu\llcorner\mathrm{M}$, c.f. 2.25) e, para todo $x \in \mathrm{M}$, existe um $k$-subespaço vetorial $\pi_{x}$ de $\mathbb{R}^{n}$ tal que

$$
\frac{\left(\Phi_{x, r}\right)_{\#} \mu}{r^{k}} \underset{r \rightarrow 0}{*} \mathcal{H}^{k}\left\llcorner\pi_{x}\right.
$$

Então $\mathrm{M}$ é k-localmente retificável e $\mu=\mathcal{H}^{k}\llcorner\mathrm{M}$.

Demonstração.

1) Para todo $x \in \mathrm{M}$,

$$
\lim _{r \rightarrow 0^{+}} \frac{\mu(\mathbb{B}(x, r))}{\alpha(k) r^{k}}=1
$$

$\mathrm{e}$

$$
\lim _{r \rightarrow 0^{+}} \frac{\mu\left(\mathbb{B}(x, r) \backslash\left[x+K\left(\pi_{x}, 1\right)\right]\right)}{\alpha(k) r^{k}}=0 .
$$

De fato, como $\pi_{x} \cap \partial \mathbb{B}^{n}$ é uma subvariedade $k$-1-dimensional mergulhada em $\mathbb{R}^{n}$, a mesma tem dimensão de Hausdorff $k-1$ em vista do exemplo 4.4. Portanto, $\mathcal{H}^{k}\left\llcorner\pi_{x}\left(\partial \mathbb{B}^{n}\right)=0\right.$. Assim, segue da hipótese do teorema e do teorema 2.89 que

$$
\begin{aligned}
\alpha(k) & =\mathcal{H}^{k}\left\llcorner\pi_{x}\left(\mathbb{B}^{n}\right)=\right. \\
& =\lim _{r \rightarrow 0^{+}} \frac{\left(\Phi_{x, r}\right)_{\#} \mu\left(\mathbb{B}^{n}\right)}{r^{k}}= \\
& =\lim _{r \rightarrow 0^{+}} \frac{\mu(\mathbb{B}(x, r))}{r^{k}},
\end{aligned}
$$

o que prova (4.13). Analogamente, $\mathcal{H}^{k}\left\llcorner\pi_{x}\left(\partial\left[\mathbb{B}^{n} \backslash K\left(\pi_{x}, 1\right)\right]\right)=0\right.$ e $\pi_{x} \cap\left[\mathbb{B}^{n} \backslash K\left(\pi_{x}, 1\right)\right]=\emptyset$, 
donde

$$
\begin{aligned}
0 & =\mathcal{H}^{k}\left\llcorner\pi_{x}\left(\mathbb{B}^{n} \backslash K\left(\pi_{x}, 1\right)\right)=\right. \\
& =\lim _{r \rightarrow 0^{+}} \frac{\left(\Phi_{x, r}\right)_{\#} \mu\left(\mathbb{B}^{n} \backslash K\left(\pi_{x}, 1\right)\right)}{r^{k}}= \\
& =\lim _{r \rightarrow 0^{+}} \frac{\mu\left(\mathbb{B}(x, r) \backslash\left[x+K\left(\pi_{x}, 1\right)\right]\right)}{r^{k}},
\end{aligned}
$$

o que prova (4.14).

2) Afirmação 1: Para todo $V \subset \mathbb{R}^{n}$ aberto, tem-se $\mathcal{H}^{k}\llcorner\mathrm{M}(V) \leq \mu(V)$.

Com efeito, para todo $x \in \mathrm{M} \cap V, \mathbb{B}(x, r) \subset V$ para $r$ suficientemente pequeno; além disso, como $\mu$ é concentrada em $\mathrm{M}$, temos $\mu=\mu\llcorner\mathrm{M}$. Portanto, para todo $x \in \mathrm{M} \cap V$,

$$
\begin{aligned}
\Theta^{k}(\mu, \mathrm{M} \cap V, x) & =\lim _{r \rightarrow 0^{+}} \frac{\mu(\mathrm{M} \cap V \cap \mathbb{B}(x, r))}{\alpha(k) r^{k}}= \\
& =\lim _{r \rightarrow 0^{+}} \frac{\mu\llcorner\mathrm{M}(\mathbb{B}(x, r))}{\alpha(k) r^{k}}= \\
& =\lim _{r \rightarrow 0^{+}} \frac{\mu(\mathbb{B}(x, r)) \stackrel{(4.13)}{=} 1 .}{\alpha(k) r^{k}} \stackrel{ }{=}=
\end{aligned}
$$

Daí, pelo teorema de comparação de densidade 2.68, segue-se que $\mathcal{H}^{k}\left\llcorner\mathrm{M}(V)=\mathcal{H}^{k}(\mathrm{M} \cap V) \leq\right.$ $\mu(\mathrm{M} \cap V)=\mu(V)$, como afirmado.

3) Afirmação 2: $\mathcal{H}^{k}\left\llcorner\mathrm{M}\right.$ é uma medida de Radon em $\mathbb{R}^{n}$.

De fato, como $\mu$ é finita em todo aberto relativamente compacto (pois é de Radon), concluise da afirmação 1 que a medida Borel-regular $\mathcal{H}^{k}\llcorner M$ também finita nos abertos relativamente compactos, portanto finita em todos os compactos de $\mathbb{R}^{n}$, logo é de Radon, em vista da observação 2.24.2).

4) Segue das afirmações 1 e 2 que, para todo $A \subset \mathbb{R}^{n}, \mathcal{H}^{k}\llcorner\mathrm{M}(A) \leq \mu(A)$. Além disso, do teorema de comparação de densidade 2.68 e de (4.13) segue, para todo $A \subset \mathrm{M}, \mu(A) \leq 2^{k} \mathcal{H}^{k}(A)$. Portanto, para todo $A \subset \mathbb{R}^{n}$,

$$
\mathcal{H}^{k}\left\llcorner\mathrm{M}(A) \leq \mu(A) \leq 2^{k} \mathcal{H}^{k}\llcorner\mathrm{M}(A) .\right.
$$

Em particular, $\mu$ e $\mathcal{H}^{k}\left\llcorner\mathrm{M}\right.$ são mutuamente absolutamente contínuas, i.e. $\mathcal{H}^{k}\llcorner\mathrm{M} \ll \mu$ e $\mu \ll$ $\mathcal{H}^{k}\llcorner\mathrm{M}$.

5) Denote por $\operatorname{Gr}(n, k)$ a grasmanniana dos $k$ subespaços vetoriais de $\mathbb{R}^{n}$. Identificando cada $k$ subespaço $\pi \in G r(n, k)$ com a projeção ortogonal $\mathrm{pr}_{\pi}$, podemos metrizar $G r(n, k)$ por, $\forall \pi, \sigma \in$ $\operatorname{Gr}(n, k)$,

$$
d(\pi, \sigma):=\left\|\operatorname{pr}_{\pi}-\operatorname{pr}_{\sigma}\right\|=\sup \left\{\left\|\mathrm{pr}_{\pi} \cdot x-\operatorname{pr}_{\sigma} \cdot x\right\| \mid x \in \mathbb{B}^{n}\right\} .
$$

Munido desta métrica, $\operatorname{Gr}(n, k)$ é um espaço métrico compacto.

6) Afirmação 3: existe $\lambda>0$ tal que, se $\pi, \sigma \in G r(n, k)$ e $d(\pi, \sigma)<\lambda$, então

$$
K(\pi, 1) \subset K(\sigma, 2) .
$$

Supondo o contrário, obtêm-se sequências $\left(\lambda_{i}\right)_{i \in \mathbb{N}}$ em $(0,1),\left(\pi_{i}\right)_{i \in \mathbb{N}}$ e $\left(\sigma_{i}\right)_{i \in \mathbb{N}}$ em $G r(n, k)$, e $\left(w_{i}\right)_{i \in \mathbb{N}}$ em $\mathbb{S}^{n-1} \cap K\left(\pi_{i}, 1\right) \backslash K\left(\sigma_{i}, 2\right)$ tais que $\lambda_{i} \downarrow 0$ e $d\left(\pi_{i}, \sigma_{i}\right)<\lambda_{i}$ para todo $i \in \mathbb{N}$. Por compacidade, passando a subsequências, se necessário, podemos supor $\pi_{i} \rightarrow \pi$ em $\operatorname{Gr}(n, k)$ e $w_{i} \rightarrow w$ em $\mathbb{S}^{n-1}$; como $\lambda_{i} \rightarrow 0$, segue $\sigma_{i} \rightarrow \pi$ em $\operatorname{Gr}(n, k)$. Tem-se: 
- $\forall i \in \mathbb{N},\left\|\operatorname{pr}_{\sigma_{i}}^{\perp} \cdot w_{i}\right\|>2\left\|\operatorname{pr}_{\sigma_{i}} \cdot w_{i}\right\|$; tomando $\lim _{i \rightarrow \infty}$, conclui-se que $\left\|\operatorname{pr}_{\pi}^{\perp} \cdot w\right\| \geq 2\left\|\mathrm{pr}_{\pi} \cdot w\right\|$.

- $\forall i \in \mathbb{N},\left\|\operatorname{pr}_{\pi_{i}}^{\perp} \cdot w_{i}\right\| \leq\left\|\operatorname{pr}_{\pi_{i}} \cdot w_{i}\right\| ;$ tomando $\lim _{i \rightarrow \infty}$, conclui-se que $\left\|\operatorname{pr}_{\pi}^{\perp} \cdot w\right\| \leq\left\|\operatorname{pr}_{\pi} \cdot w\right\|$.

- Em vista dos dois itens anteriores, conclui-se que $w=0$, chegando-se a uma contradição, pois $\|w\|=1$.

7) Afirmação 4: existe $\lambda>0$ tal que, se $\sigma \in G r(n, k)$ e $w \in \mathbb{R}^{n} \backslash K(\sigma, 3)$, então

$$
\mathbb{B}(w, \lambda\|w\|) \cap K(\sigma, 2)=\emptyset .
$$

Com efeito, tome $\lambda>0$ tal que a igualdade acima valha sempre que $w \in \mathbb{R}^{n} \backslash K(\sigma, 3)$ com $\|w\|=1$; a existência de um tal $\lambda$ decorre do fato de que $\overline{\mathbb{S}^{n-1} \backslash K(\sigma, 3)} \subset \mathbb{R}^{n}$ é um compacto disjunto do fechado $K(\sigma, 2)$. Dado $w \in \mathbb{R}^{n} \backslash K(\sigma, 3)$, se existir $x \in \mathbb{B}(w, \lambda\|w\|) \cap K(\sigma, 2)$, então $\frac{x}{\|w\|} \in \mathbb{B}\left(\frac{w}{\|w\|}, \lambda\right) \cap K(\sigma, 2)$ e $\frac{w}{\|w\|} \in \mathbb{R}^{n} \backslash K(\sigma, 3)$, o que não pode ocorrer, concluindo-se a prova da afirmação 4 .

8) Fixe $\lambda \in(0,1)$ satisfazendo as afirmações 3 e 4 . Como $G r(n, k)$ é compacto, existe $N \in \mathbb{N}$ e $\left(\sigma_{i}\right)_{1 \leq i \leq N}$ em $\operatorname{Gr}(n, k)$ tal que

$$
G r(n, k)=\bigcup_{i=1}^{N} \mathbb{U}\left(\sigma_{i}, \lambda\right) .
$$

Para cada $\sigma \in G r(n, k)$, definimos

$$
M(\sigma):=\left\{x \in \mathrm{M} \mid \pi_{x} \in \mathbb{U}(\sigma, \lambda)\right\} .
$$

Assim, decorre de (4.15) que

$$
\mathrm{M}=\cup_{i=1}^{N} \mathrm{M}\left(\sigma_{i}\right)
$$

9) Afirmação 5: sejam $\mathrm{M}^{\prime} \subset \mathrm{M}$ limitado e $\sigma \in G r(n, k)$. Suponha que os limites

$$
\begin{aligned}
& \lim _{r \rightarrow 0^{+}} \frac{\mu(\mathbb{B}(x, r))}{\alpha(k) r^{k}}=1, \\
& \lim _{r \rightarrow 0^{+}} \frac{\mu(\mathbb{B}(x, r) \backslash[x+K(\sigma, 2)])}{\alpha(k) r^{k}}=0
\end{aligned}
$$

existam uniformemente com respeito a $x \in \mathrm{M}^{\prime}$. Então $\mathrm{M}^{\prime}$ pode ser coberto por um número finito de imagens de conjuntos limitados por aplicações Lipschitz $\mathbb{R}^{k} \rightarrow \mathbb{R}^{n}$.

Com efeito, segue da condição acima que, para todo $\epsilon>0$, existe $\delta=\delta(\epsilon)>0$ tal que, para todo $x \in \mathrm{M}^{\prime}$ e todo $r \in(0,2 \delta)$,

$$
\begin{gathered}
\mu(\mathbb{B}(x, r)) \geq(1-\epsilon) \alpha(k) r^{k}, \\
\mu(\mathbb{B}(x, r) \backslash[x+K(\sigma, 2)]) \leq \epsilon \alpha(k) r^{k} .
\end{gathered}
$$

Verifiquemos que é possível escolher $\epsilon=\epsilon(k, \lambda)$ de modo a valer, para todo $x \in M^{\prime}$,

$$
\mathbb{B}(x, \delta(\epsilon)) \cap \mathrm{M}^{\prime} \subset x+K(\sigma, 3) .
$$

De fato, dado $\epsilon>0$, se $\exists x \in \mathrm{M}^{\prime}, \exists y \in \mathbb{B}(x, \delta(\epsilon)) \cap \mathrm{M}^{\prime}$ com $y-x \notin K(\sigma, 3)$, segue da escolha de $\lambda$ no passo 8 e da afirmação 4 que $\mathbb{B}(y-x, \lambda\|y-x\|) \cap K(\sigma, 2)=\emptyset$ ou, equivalentemente,

$$
\mathbb{B}(y, \lambda\|y-x\|) \subset \mathbb{R}^{n} \backslash[x+K(\sigma, 2)] .
$$


Como $\lambda \in(0,1)$, temos $\mathbb{B}(y, \lambda\|y-x\|) \subset \mathbb{B}(x, 2\|y-x\|)$, donde, por (4.21),

$$
\mathbb{B}(y, \lambda\|y-x\|) \subset \mathbb{B}(x, 2\|y-x\|) \backslash[x+K(\sigma, 2)] .
$$

Daí, de (4.19) com $r=2\|y-x\|<2 \delta(\epsilon)$, de (4.18) com $r=\lambda\|y-x\|<2 \delta(\epsilon)$ e de (4.22), obtém-se

$$
\epsilon \alpha(k) 2^{k}\|y-x\|^{k} \geq(1-\epsilon) \alpha(k) \lambda^{k}\|y-x\|^{k},
$$

ou, equivalentemente,

$$
2^{k} \epsilon \geq(1-\epsilon) \lambda^{k}
$$

Portanto, para $\epsilon=\epsilon(k, \lambda)$ suficientemente pequeno, obtém-se uma contradição. Para esta escolha de $\epsilon=\epsilon(k, \lambda)$, (4.20) é verificada para todo $x \in \mathrm{M}^{\prime}$, como afirmado. Daí, como $M^{\prime} \subset \mathbb{R}^{n}$ é limitado, o lema 4.20 se aplica e podemos cobrir $\mathrm{M}^{\prime}$ com um número finito de imagens de conjuntos limitados por aplicações Lipschitz $\mathbb{R}^{k} \rightarrow \mathbb{R}^{n}$, o que conclui a prova da afirmação 5 .

10) Fixe $\sigma \in G r(n, k)$ e considere as famílias $\left(f_{r}\right)_{r>0}$ e $\left(g_{r}\right)_{r>0}$ de funções $\mathbb{R}^{n} \rightarrow \mathbb{R}$ dadas por, para todo $x \in \mathbb{R}^{n}$,

$$
f_{r}(x):=\frac{\mu(\mathbb{B}(x, r))}{\alpha(k) r^{k}} \text { e } g_{r}(x):=\frac{\mu(\mathbb{B}(x, r) \backslash[x+K(\sigma, 2)])}{\alpha(k) r^{k}} .
$$

Afirmação 6: para todo $r>0, f_{r}$ e $g_{r}$ são borelianas. Além disso, para $x \in \mathbb{R}^{n}$ fixo, $r \in(0, \infty) \mapsto$ $f_{r}(x)$ e $r \in(0, \infty) \mapsto g_{r}(x)$ são contínuas à direita.

De fato, para $r>0$ fixo, sejam $A:=\left\{(x, y) \in \mathbb{R}^{n} \times \mathbb{R}^{n} \mid y \in \mathbb{B}(x, r)\right\}=\left\{(x, y) \in \mathbb{R}^{n} \times \mathbb{R}^{n} \mid\right.$ $\|x-y\| \leq r\}$ e $B=\left\{(x, y) \in \mathbb{R}^{n} \times \mathbb{R}^{n} \mid y \in \mathbb{B}(x, r) \backslash[x+K(\sigma, 2)]\right\}=\left\{(x, y) \in \mathbb{R}^{n} \times \mathbb{R}^{n} \mid\right.$ $\|x-y\| \leq r$ e $\left.\left\|\operatorname{pr}_{\sigma}^{\perp} \cdot(y-x)\right\|>2\left\|\mathrm{pr}_{\sigma} \cdot(y-x)\right\|\right\}$. Então $A$ e $B$ são borelianos de $\mathbb{R}^{n} \times \mathbb{R}^{n}$. Como $\mu$ é uma medida boreliana $\sigma$-finita em $\mathbb{R}^{n}$ (pois é de Radon e $\mathbb{R}^{n}$ é $\sigma$-compacto), segue do teorema de Tonelli clássico que as funções (recorde a notação para seções fixada em 2.2.2) $\mathbb{R}^{n} \rightarrow \mathbb{R}$ dadas por $x \in \mathbb{R}^{n} \mapsto \mu\left(A_{x}\right)=\int \chi_{A}(x, y) \mathrm{d} \mu(y)$ e $x \in \mathbb{R}^{n} \mapsto \mu\left(B_{x}\right)=\int \chi_{B}(x, y) \mathrm{d} \mu(y)$ são borelianas. Portanto, também são borelianas as funções $x \in \mathbb{R}^{n} \mapsto f_{r}(x)=\frac{\mu\left(A_{x}\right)}{\alpha(k) r^{k}}$ e $x \in \mathbb{R}^{n} \mapsto g_{r}(x)=$ $\frac{\mu\left(B_{x}\right)}{\alpha(k) r^{k}}$. Além disso, para $x \in \mathbb{R}^{n}$ fixo, o denominador de ambas estas frações é contínuo como função de $r$ e os numeradores são contínuos à direita em virtude da continuidade para baixo da medida de Radon $\mu$; portanto, $r \in(0, \infty) \mapsto f_{r}(x)$ e $r \in(0, \infty) \mapsto g_{r}(x)$ são contínuas à direita, o que conclui a prova da afirmação 6 .

11) Afirmação 7: para cada $\sigma \in G r(n, k)$, existe um conjunto $\mathcal{H}^{k}$-nulo $Z_{\sigma} \subset \mathrm{M}(\sigma)$ tal que $\mathrm{M}(\sigma) \backslash Z_{\sigma}$ pode ser coberto por uma união enumerável de imagens de conjuntos limitados por aplicações Lipschitz $\mathbb{R}^{k} \rightarrow \mathbb{R}^{n}$.

Para provar esta afirmação, basta considerar o caso em que $M$ é limitado; no caso geral, basta escrever $\mathrm{M}$ como união enumerável de borelianos limitados e aplicar o caso particular a cada um dos tais limitados. Assim, $\mathrm{M}(\sigma) \subset \mathrm{M}$ é limitado; e, como $\mu$ é de Radon, logo finita nos compactos, tem-se $\mu(\mathrm{M}(\sigma))<\infty$. Portanto, $\mu\llcorner\mathrm{M}(\sigma)$ é uma medida boreliana (em vista da proposição 2.15.i) finita. Além disso:

- $\mathrm{M}(\sigma)$ é $\mu\left\llcorner\mathrm{M}(\sigma)\right.$-mensurável e as funções das famílias $\left(f_{r}\right)_{r>0}$ e $\left(g_{r}\right)_{r>0}$ definidas no item anterior são borelianas (logo $\mu\llcorner\mathrm{M}(\sigma)$-mensuráveis) e contínuas à direita como funções de $r>0$ (em vista da afirmação 6).

- Pelo passo $1, f_{r}$ é pontualmente convergente para a função constante e igual a $1 \mathrm{em} \mathrm{M} \supset$ $\mathrm{M}(\sigma)$.

- Pela escolha de $\lambda$ no passo 8 e pela afirmação 3, tem-se, para todo $x \in \mathrm{M}(\sigma), x+K\left(\pi_{x}, 1\right) \subset$ $x+K(\sigma, 2), \operatorname{logo} \mathbb{B}(x, r) \backslash[x+K(\sigma, 2)] \subset \mathbb{B}(x, r) \backslash\left[x+K\left(\pi_{x}, 1\right)\right]$. Portanto, para todo 
$x \in \mathrm{M}(\sigma)$ e todo $r>0$,

$$
g_{r}(x) \leq \frac{\mu\left(\mathbb{B}(x, r) \backslash\left[x+K\left(\pi_{x}, 1\right)\right]\right)}{\alpha(k) r^{k}},
$$

e do passo 1 conclui-se que a família $\left(g_{r}\right)_{r>0}$ é pontualmente convergente para zero em $\mathrm{M}(\sigma)$.

Estão verificadas, portanto, as hipóteses do teorema de Severini-Egorov para famílias de funções 2.44 para a medida $\mu\left\llcorner\mathrm{M}(\sigma)\right.$ e para as famílias de funções $\mu\left\llcorner\mathrm{M}(\sigma)\right.$-mensuráveis $\left(f_{r}\right)_{r>0}$ e $\left(g_{r}\right)_{r>0}$, as quais são pontualmente convergentes em $\mathrm{M}(\sigma)$. Aplicando o referido teorema, podemos construir indutivamente uma sequência disjunta $\left(M_{i}\right)_{i \in \mathbb{N}}$ de subconjuntos $\mu\llcorner\mathrm{M}(\sigma)$ mensuráveis de $\mathrm{M}(\sigma)$ tal que, para cada $i \in \mathbb{N}, \mu\left(\mathrm{M}(\sigma) \backslash \cup_{j \leq i} M_{j} \leq \frac{1}{i}\right)$ e as famílias $\left(f_{r}\right)_{r>0}$ e $\left(g_{r}\right)_{r>0}$ sejam uniformemente convergentes em $M_{i}$, respectivamente, para 1 e 0 . Como cada $M_{i} \subset \mathrm{M}(\sigma) \subset \mathrm{M}$ é limitado, segue da afirmação 5 que $M_{i}$ pode ser coberto com um número finito de imagens de conjuntos limitados por aplicações Lipschitz $\mathbb{R}^{k} \rightarrow \mathbb{R}^{n}$. Logo, $\cup_{i \in \mathbb{N}} M_{i} \subset \mathrm{M}(\sigma)$ pode ser coberto por uma união enumerável de imagens de conjuntos limitados por aplicações Lipschitz $\mathbb{R}^{k} \rightarrow \mathbb{R}^{n}$. Finalmente, pondo $Z_{\sigma}:=\mathrm{M}(\sigma) \backslash \cup_{i \in \mathbb{N}} M_{i}$, temos $\mu\left(Z_{\sigma}\right)=0$; daí, pelo passo $4, \mathcal{H}^{k}\left(Z_{\sigma}\right)=0$, o que conclui a prova da afirmação 7 .

12) Como $\mathbf{M}=\cup_{i=1}^{N} \mathbf{M}\left(\sigma_{i}\right)$, por (4.16), aplicando-se a afirmação 7 para cada $\mathbf{M}\left(\sigma_{i}\right)$ conclui-se que $\mathbf{M}$ é $k$-enumeravelmente retificável. E, como $\mathcal{H}^{k}\llcorner\mathrm{M}$ é uma medida de Radon, pelo passo 3, segue que $\mathrm{M}$ é $k$-localmente retificável.

13) Resta verificar que $\mu=\mathcal{H}^{k}\left\llcorner M\right.$. Dos passos 3 e 4 , sabemos que $\mathcal{H}^{k}\llcorner M$ é uma medida de Radon em $\mathbb{R}^{n}$ e que $\mathcal{H}^{k}\llcorner\mathrm{M}$ e $\mu$ são mutuamente absolutamente contínuas. Além disso, como $\mathrm{M}$ é $k$-localmente retificável, segue-se do teorema 4.10 que, para $\mathcal{H}^{k}$-q.t. $x \in \mathrm{M}$,

$$
\lim _{r \rightarrow 0^{+}} \frac{\mathcal{H}^{k}(M \cap \mathbb{B}(x, r))}{\alpha(k) r^{k}}=1
$$

Como $\mu \ll \mathcal{H}^{k}\llcorner\mathrm{M}$, a igualdade acima também vale $\mu$-q.s. em $\mathrm{M}$. Portanto, em vista de (4.13), conclui-se que, para $\mu$-q.t. $x \in \mathrm{M}$,

$$
\begin{aligned}
\Theta^{\mu}\left(\mathcal{H}^{k}\llcorner\mathrm{M}, x)\right. & =\lim _{r \rightarrow 0^{+}} \frac{\mathcal{H}^{k} \operatorname{LM}(\mathbb{B}(x, r))}{\mu(\mathbb{B}(x, r))}= \\
& =\lim _{r \rightarrow 0^{+}} \frac{\frac{\mathcal{H}^{k}\llcorner\mathrm{M}(\mathbb{B}(x, r))}{\alpha(k) r^{k}}}{\frac{\mu(\mathbb{B}(x, r))}{\alpha(k) r^{k}}}=1 .
\end{aligned}
$$

Como $\mu$ é concentrada em $\mathrm{M}$, conclui-se que $\Theta^{\mu}\left(\mathcal{H}^{k}\llcorner\mathrm{M}, \cdot)=1 \mu\right.$-q.s. em $\mathbb{R}^{n}$. Daí, do teorema de diferenciação de Lebesgue-Besicovitch-Radon-Nikodym 2.81 e do fato de que $\mathcal{H}^{k}\llcorner M \ll \mu$, conclui-se que $\mu$ e $\mathcal{H}^{k}\left\llcorner\mathrm{M}\right.$ coincidem nos borelianos de $\mathbb{R}^{n}$. Como são ambas medidas de Radon em $\mathbb{R}^{n}$, logo Borel-regulares, segue $\mu=\mathcal{H}^{k}\llcorner\mathrm{M}$, como afirmado. 


\section{Capítulo 5}

\section{Formula da Área e Coárea em Conjuntos Retificáveis}

Nosso objetivo, neste capítulo, é enunciar e demonstrar versões das fórmulas da área e da coárea para aplicações Lipschitz definidas em conjuntos retificáveis, generalizando-se os teoremas correspondentes enunciados no capítulo 3 para o caso euclidiano. Isso será feito nos teoremas 5.7 (fórmula da área) e 5.9 (fórmula da coárea).

\subsection{Fórmula da Área para Conjuntos Retificáveis}

Inicialmente, como aquecimento, e para ganhar alguma intuição geométrica antes de discutir o caso geral, demonstraremos uma versão da fórmula da área para aplicações de classe $C^{1}$ definidas em subvariedades mergulhadas $k$-dimensionais de classe $C^{1}$ em $\mathbb{R}^{n}$. O leitor que se considerar suficientemente aquecido poderá pular diretamente para o caso geral, a começar com a definição 5.4.

Definição 5.1 (diferenciabilidade tangencial em subvariedades). Sejam M uma $k$-subvariedade mergulhada $C^{1}$ em $\mathbb{R}^{n}, x \in \mathrm{M}, \mathcal{U}$ uma vizinhança aberta de $x$ em $\mathbb{R}^{n}$ e $f: \mathcal{U} \rightarrow \mathbb{R}^{m}$. Diz-se que $f$ é tangencialmente diferenciável em $x$ com respeito a $\mathrm{M}$ se, para todo $v \in \mathrm{T}_{x} \mathrm{M}$,

$$
\exists \lim _{t \rightarrow 0} \frac{f(x+t v)-f(x)}{t},
$$

e o limite seja linear como função de $v \in \mathrm{T}_{x} \mathrm{M}$ e uniforme na esfera unitária $\left\{v \in \mathrm{T}_{x} \mathrm{M} \mid\|v\|=1\right\}$.

Caso $f$ seja tangencialmente diferenciável em $x$ com respeito a $\mathrm{M}$, definimos a derivada tangencial de $f$ em $x$ com respeito a $\mathrm{M}, \mathrm{D}^{\mathrm{M}} f(x) \in \mathrm{L}\left(\mathrm{T}_{x} \mathrm{M}, \mathbb{R}^{m}\right) \equiv \mathbb{R}^{m} \otimes \mathrm{T}_{x} \mathrm{M}^{*}$ por

$$
\mathrm{D}^{\mathrm{M}} f(x) \cdot v:=\lim _{t \rightarrow 0} \frac{f(x+t v)-f(x)}{t} .
$$

Também definimos, nesse caso:

- o gradiente tangencial de $f$ em $x$ com respeito a $\mathrm{M}$ como sendo o elemento $\nabla^{\mathrm{M}} f(x)$ de $\mathbb{R}^{m} \otimes$ $\mathrm{T}_{x} \mathrm{M}$ que corresponde a $\mathrm{D}^{\mathrm{M}} f(x)$ através da identificação $\mathbb{R}^{m} \otimes \mathrm{T}_{x} \mathrm{M}^{*} \equiv \mathbb{R}^{m} \otimes \mathrm{T}_{x} \mathrm{M}$ induzida pelo produto interno de $\mathrm{T}_{x} \mathrm{M}$;

- o jacobiano tangencial de $f$ em $x$ com respeito a $\mathrm{M}$,

$$
\mathrm{J}^{\mathrm{M}} f(x):=\llbracket \mathrm{D}^{\mathrm{M}} f(x) \rrbracket=\sqrt{\operatorname{det}\left[\mathrm{D}^{\mathrm{M}} f(x)^{*} \circ \mathrm{D}^{\mathrm{M}} f(x)\right]},
$$

onde $\mathrm{D}^{\mathrm{M}} f(x)^{*} \in \mathrm{L}\left(\mathbb{R}^{m}, \mathrm{~T}_{x} \mathrm{M}\right)$ denota a transposta de $\mathrm{D}^{\mathrm{M}} f(x)$.

Observação 5.2. Com a notação da definição acima: 
1) Decorre da proposição 2.96 que $f$ é tangencialmente diferenciável em $x$ com respeito a $\mathrm{M}$ se, e somente se, a restrição de $f$ a $x+\mathrm{T}_{x} \mathrm{M}$ (identificado ao $\mathbb{R}^{k}$ por meio de alguma isometria linear, i.e. através da escolha uma base ortonormal no espaço tangente) for diferenciável no sentido de Fréchet em $x$.

2) Em vista do item anterior, a definição de diferenciabilidade tangencial generaliza a noção de "derivada direcional" (i.e. as derivadas direcionais em $x$ coincidem com as derivadas tangenciais no caso em que $k=1)$.

3) Se $f$ for derivável no sentido de Fréchet em $x$, é claro que $f$ é tangencialmente diferenciável em $x$ com respeito a $\mathrm{M}$, e a derivada tangencial $\mathrm{D}^{\mathrm{M}} f(x)$ coincide com a restrição de $\mathrm{D} f(x)$ a $\mathrm{T}_{x} \mathrm{M}$. Portanto, a referida derivada também coincide com a derivada em $x$ da restrição de $f$ a M (derivada no sentido do Cálculo em Variedades) e, em particular, a derivada tangencial só depende da restrição de $f$ a M. Além disso, podemos calcular $\nabla^{\mathrm{M}} f(x)$ através da seguinte fórmula, dada $\left(\nu_{i}\right)_{1 \leq i \leq n}$ base ortonormal adaptada a $\mathrm{T}_{x} \mathrm{M} \oplus \mathrm{T}_{x} \mathrm{M}^{\perp}$ :

$$
\nabla^{\mathrm{M}} f(x)=\sum_{i=1}^{k}\left(\mathrm{D} f(x) \cdot \nu_{i}\right) \otimes \nu_{i}=\nabla f(x)-\sum_{i=k+1}^{n}\left(\mathrm{D} f(x) \cdot \nu_{i}\right) \otimes \nu_{i} .
$$

4) Se $f: M \rightarrow \mathbb{R}^{m}$ for derivável em $x$ (no sentido do Cálculo em Variedades), podemos estender $f$ a uma função derivável no sentido de Fréchet em $x$, definida em alguma vizinhança aberta $\mathcal{U}$ de $x$, de modo que o item anterior se aplica a esta extensão; como visto no item anterior, a derivada tangencial desta extensão em $x$ com respeito a $M$ coincide com a derivada de $f$ em $x$. Em particular, o jacobiano tangencial em $x$ desta extensão coincide com o jacobiano de $f$ em $x$, i.e. com $\llbracket \mathrm{T}_{x} f \rrbracket$, onde $\mathrm{T}_{x} f: \mathrm{T}_{x} \mathrm{M} \rightarrow \mathbb{R}^{m}$ denota a aplicação tangente de $f$ em $x$. Por esta razão, usaremos a notação $\mathrm{J}^{\mathrm{M}} f$ para denotar o jacobiano de $f$, i.e. para todo $x \in \mathrm{M}$,

$$
\mathrm{J}^{\mathrm{M}} f(x):=\llbracket \mathrm{T}_{x} f \rrbracket .
$$

Teorema 5.3 (Fórmula da Área em Subvariedades Mergulhadas). Sejam M uma k-subvariedade mergulhada $\mathrm{C}^{1}$ em $\mathbb{R}^{n}, k \leq m, f: \mathrm{M} \rightarrow \mathbb{R}^{m}$ de classe $\mathrm{C}^{1}$ e injetiva. Então, para todo $A \in \mathscr{B}_{\mathrm{M}}$,

$$
\mathcal{H}^{k}(f(A))=\int_{A} \mathrm{~J}^{\mathrm{M}} f \mathrm{~d} \mathcal{H}^{k} .
$$

Demonstração. É suficiente considerar o caso em que $A$ está contido na imagem de um sistema de coordenadas $g: \mathcal{U} \subset \mathbb{R}^{k} \rightarrow \mathrm{M}$; no caso geral, podemos decompor $A$ numa união disjunta enumerável de borelianos de $\mathrm{M}$, cada um dos quais contido numa vizinhança coordenada.

Seja $\widetilde{A}:=g^{-1}(A) \subset \mathbb{R}^{k}$. Então $\widetilde{A} \in \mathscr{B}_{\mathbb{R}^{k}}$ e $f \circ g: \mathcal{U} \rightarrow \mathbb{R}^{m}$ é de classe $C^{1}$, portanto localmente Lipschitz. Então segue da fórmula da área 3.12.i) aplicada a $f \circ g$, que

$$
\mathcal{H}^{k}(f(A))=\mathcal{H}^{k}(f \circ g(\widetilde{A}))=\int_{\widetilde{A}} \mathrm{~J}(f \circ g) \mathrm{d} \mathcal{H}^{k} .
$$

Por outro lado, segue da regra da cadeia que, para todo $x \in \mathcal{U}, \mathrm{D}(f \circ g)(x)=\mathrm{T}_{g(x)} f \circ \mathrm{D} g(x)$. Fixando-se uma isometria linear $\mathrm{T}_{x} \mathrm{M} \equiv \mathbb{R}^{k}$ (a qual não altera os jacobianos de $\mathrm{D} g(x) \in \mathrm{L}\left(\mathbb{R}^{k}, \mathrm{~T}_{x} \mathrm{M}\right.$ ) e $\mathrm{T}_{g(x)} f \in \mathrm{L}\left(\mathrm{T}_{x} \mathrm{M}, \mathbb{R}^{m}\right)$ ), segue da proposição 2.105.i) que

$$
\begin{aligned}
\mathrm{J}(f \circ g)(x) & =\llbracket \mathrm{T}_{g(x)} f \circ \mathrm{D} g(x) \rrbracket= \\
& =\llbracket \mathbf{T}_{g(x)} f \rrbracket \llbracket \mathrm{D} g(x) \rrbracket= \\
& =\mathrm{J}^{\mathrm{M}} f(g(x)) \mathrm{J} g(x) .
\end{aligned}
$$

Daí, aplicando-se o teorema de mudança de variáveis 3.12.ii) para a aplicação localmente Lips- 
chitz $g: \mathcal{U} \rightarrow \mathbb{R}^{n}$, obtém-se

$$
\begin{aligned}
\int_{\widetilde{A}} \mathrm{~J}(f \circ g)(x) \mathrm{d} \mathcal{H}^{k}(x) & =\int_{\widetilde{A}} \mathrm{~J}^{\mathrm{M}} f(g(x)) \mathrm{J} g(x) \mathrm{d} \mathcal{H}^{k}(x)= \\
& =\int_{\underbrace{g(\widetilde{A})}_{=A}} \mathrm{~J}^{\mathrm{M}} f \mathrm{~d} \mathcal{H}^{k} .
\end{aligned}
$$

De (5.2) e (5.3), segue a tese.

Definição 5.4 (diferenciabilidade tangencial em conjuntos retificáveis). Sejam $\mathrm{M} \subset \mathbb{R}^{n}$ um conjunto $k$-localmente retificável e $f: \mathbb{R}^{n} \rightarrow \mathbb{R}^{m}$ Lipschitz. Diz-se que $f$ é tangencialmente diferenciável em $x \in \mathrm{M}$ com respeito a $\mathrm{M}$ se $\mathrm{M}$ admitir espaço tangente aproximado em $x$ e se a restrição de $f$ a $x+\mathrm{T}_{x} \mathrm{M}$ for diferenciável em $x$ no sentido de Fréchet. Caso afirmativo, usamos as mesmas notações da definição 5.1 e da observação 5.2 , i.e. $\mathrm{D}^{\mathrm{M}} f(x)$ para a derivada tangencial de $f$ em $x, \nabla^{\mathrm{M}} f(x)$ para o gradiente tangencial de $f$ em $x$ e $\mathrm{J}^{\mathrm{M}} f(x)$ para o jacobiano tangencial de $f$ em $x$ com respeito a M.

Lema 5.5. Sejam $\mathrm{M}=g(E) \subset \mathbb{R}^{n}$ uma imagem Lipschitz regular $k$-dimensional definida pelo par $(g, E)$ e $f: \mathbb{R}^{n} \rightarrow \mathbb{R}^{m}$ Lipschitz. Então, para $\mathcal{H}^{k}$-quase todo $x \in \mathrm{M}$, $f \circ g$ é diferenciável em $g^{-1}(x)$, $f$ é tangencialmente diferenciável em $x$ com respeito a $\mathrm{M} e$

$$
\mathrm{D}^{\mathrm{M}} f(x)=\mathrm{D}(f \circ g)\left(g^{-1}(x)\right) \circ \mathrm{D} g\left(g^{-1}(x)\right)^{-1} .
$$

\section{Demonstração.}

1) Segue da observação 4.16 que, para todo $x \in \mathrm{M}$, o espaço tangente aproximado em $x$ existe e é dado por

$$
\mathrm{T}_{x} \mathrm{M}=\mathrm{D} g\left(g^{-1}(x)\right) \cdot \mathbb{R}^{k} .
$$

Além disso, segue da definição de imagem Lipschitz regular que $\mathrm{D} g\left(g^{-1}(x)\right): \mathbb{R}^{k} \rightarrow \mathbb{R}^{n}$ é um isomorfismo linear sobre $\mathrm{T}_{x} \mathrm{M}$.

2) A composta $f \circ g: \mathbb{R}^{k} \rightarrow \mathbb{R}^{m}$ é Lipschitz e, portanto, pelo teorema de Rademacher, é diferenciável em $\mathcal{L}^{k}$-quase todo $z \in \mathbb{R}^{k}$. Em particular, é diferenciável em $\mathcal{L}^{k}$-quase todo $z \in E$. Seja $D \subset E$ o conjunto dos pontos de $E$ nos quais $f \circ g$ é diferenciável; provaremos que $f$ é tangencialmente diferenciável com respeito a $\mathrm{M}$ em $g(D)$, e que sua derivada tangencial nos pontos desse conjunto é dada pela fórmula do enunciado. Como $\mathcal{L}^{k}(E \backslash D)=0$ e $g$ é Lipschitz, tem-se $\mathcal{H}^{k}(\mathrm{M} \backslash g(D))=$ $\mathcal{H}^{k}(g(E \backslash D))=0$, e daí seguirá a tese.

3) Seja $z \in D$. Provemos que $f$ é tangencialmente diferenciável em $x=g(z)$ e que sua derivada tangencial nesse ponto é dada pela fórmula enunciada. De fato, para todo $w \in \mathbb{R}^{k} \backslash\{0\}$ e $v=\mathrm{D} g(z) \cdot w \in \mathrm{T}_{x} \mathrm{M}=\mathrm{D} g(z) \cdot \mathbb{R}^{k}$, tem-se:

$$
\begin{aligned}
& f(x+v)-f(x)-\mathrm{D}(f \circ g)\left(g^{-1}(x)\right) \cdot \mathrm{D} g\left(g^{-1}(x)\right)^{-1} \cdot v= \\
& \quad=f(g(z)+\mathrm{D} g(z) \cdot w)-f(g(z))-\mathrm{D}(f \circ g)(z) \cdot w= \\
& \quad=[f(g(z)+\mathrm{D} g(z) \cdot w)-f(g(z+w))]+[f(g(z+w))-f(g(z))-\mathrm{D}(f \circ g)(z) \cdot w] .
\end{aligned}
$$


Como $\|w\|=\left\|\mathrm{D} g(z)^{-1} \cdot v\right\| \leq\left\|\mathrm{D} g(z)^{-1}\right\|\|v\|$, tem-se $\|v\| \geq\left\|\mathrm{D} g(z)^{-1}\right\|^{-1}\|w\|$. Portanto,

$$
\begin{gathered}
\frac{\left\|f(x+v)-f(x)-\mathrm{D}(f \circ g)\left(g^{-1}(x)\right) \cdot \mathrm{D} g\left(g^{-1}(x)\right)^{-1} \cdot v\right\|}{\|v\|} \leq \\
\leq\left\|\mathrm{D} g(z)^{-1}\right\|(\operatorname{Lip} f) \frac{\|g(z+w)-g(z)-\mathrm{D} g(z) \cdot w\|}{\|w\|}+ \\
\quad+\left\|\mathrm{D} g(z)^{-1}\right\| \frac{\|f(g(z+w))-f(g(z))-\mathrm{D}(f \circ g)(z) \cdot w\|}{\|w\|} .
\end{gathered}
$$

Como $w=\mathrm{D} g(z)^{-1} \cdot v$, temos $w \rightarrow 0$ se $v \rightarrow 0$; daí, pela diferenciabilidade de $g$ e de $f \circ g$ em $z$, conclui-se que

$$
\lim _{v \rightarrow 0} \frac{\left\|f(x+v)-f(x)-\mathrm{D}(f \circ g)\left(g^{-1}(x)\right) \cdot \mathrm{D} g\left(g^{-1}(x)\right)^{-1} \cdot v\right\|}{\|v\|}=0,
$$

o que mostra que $f$ admite derivada tangencial em $x$ com respeito a $M$ e $D^{M} f(x)$ é dada pela fórmula enunciada.

Teorema 5.6 (teorema de Rademacher para funções Lipschitz em conjuntos retificáveis). Sejam $\mathrm{M} \subset \mathbb{R}^{n}$ um conjunto $k$-localmente retificável e $f: \mathbb{R}^{n} \rightarrow \mathbb{R}^{m}$ Lipschitz. Então $f$ é tangencialmente diferenciável com respeito a $\mathrm{M}$ em $\mathcal{H}^{k}$-quase todo $x \in \mathrm{M}$.

Demonstração. Seja $\mathrm{M}=\mathrm{M}_{0} \cup \bigcup_{i \in \mathbb{N}} g_{i}\left(E_{i}\right)$, com $\mathrm{M}_{0} \mathcal{H}^{k}$-nulo e, para cada $i \in \mathbb{N}, g_{i}\left(E_{i}\right)$ imagem Lipschitz regular definida pelo par $\left(g_{i}, E_{i}\right)$. É suficiente verificar que, para cada $i \in \mathbb{N}, f$ é tangencialmente diferenciável com respeito a $\mathrm{M}$ em $\mathcal{H}^{k}$-q.t. $x \in g_{i}\left(E_{i}\right)$. Como $g_{i}\left(E_{i}\right)$ é imagem Lipschitz regular (e, em particular, é $k$-localmente retificável) e $g_{i}\left(E_{i}\right)=g_{i}\left(E_{i}\right) \cap \mathrm{M}$, segue da proposição $4.19 \mathrm{com} M_{1}=g_{i}\left(E_{i}\right)$ e $M_{2}=\mathrm{M}$ que, para $\mathcal{H}^{k}$-q.t. $x \in g_{i}\left(E_{i}\right)$, os espaços tangentes aproximados $\mathrm{T}_{x}\left[g_{i}\left(E_{i}\right)\right]$ e $\mathrm{T}_{x} \mathrm{M}$ existem e coincidem. Por outro lado, pelo lema 5.5, para $\mathcal{H}^{k}$-q.t. $x \in g_{i}\left(E_{i}\right)$, a restrição de $f$ a $x+\mathrm{T}_{x}\left[g_{i}\left(E_{i}\right)\right]$ é derivável no sentido de Fréchet em $x$ (i.e. $f$ é tangencialmente diferenciável em $x$ com respeito a $\left.g_{i}\left(E_{i}\right)\right)$. Portanto, existe $D \subset g_{i}\left(E_{i}\right)$ com $\mathcal{H}^{k}\left(g_{i}\left(E_{i}\right) \backslash D\right)=0$ e tal que, para todo $x \in D, \mathrm{~T}_{x} \mathrm{M}=\mathrm{T}_{x}\left[g_{i}\left(E_{i}\right)\right]$ e a restrição de $f$ a $x+\mathrm{T}_{x} \mathrm{M}$ é derivável no sentido de Fréchet em $x$.

Teorema 5.7 (Fórmula da Área para aplicações Lipschitz em conjuntos retificáveis). Sejam M $\subset$ $\mathbb{R}^{n}$ um conjunto $k$-localmente retificável e $f: \mathbb{R}^{n} \rightarrow \mathbb{R}^{m}$ Lipschitz, com $k \leq m$. Então:

i) $\mathrm{J}^{\mathrm{M}} f$ é uma função definida $\mathcal{H}^{k}$-q.s. em $\mathrm{M}$ e $\mathcal{H}^{k}$-mensurável;

ii) a função de multiplicidade $N\left(\left.f\right|_{\mathrm{M}}\right): \mathbb{R}^{m} \rightarrow[0, \infty]$ dada por $y \mapsto \mathcal{H}^{0}\left(\mathrm{M} \cap f^{-1}\{y\}\right)$ é $\mathcal{H}^{k}$ mensurável;

iii) $\int_{\mathbb{R}^{m}} \mathcal{H}^{0}\left(\mathrm{M} \cap f^{-1}\{y\}\right) \mathrm{d} \mathcal{H}^{k}(y)=\int_{\mathrm{M}} \mathrm{J}^{\mathrm{M}} f \mathrm{~d} \mathcal{H}^{k}$.

Em particular, se $\left.f\right|_{\mathrm{M}}$ for injetiva,

$$
\mathcal{H}^{k}(f(\mathrm{M}))=\int_{\mathrm{M}} \mathrm{J}^{\mathrm{M}} f \mathrm{~d} \mathcal{H}^{k} .
$$

Demonstração.

1) Podemos supor que $\mathrm{M}=g(E)$ é uma imagem Lipschitz regular $k$-dimensional definida pelo par $(g, E)$, com $E \in \mathscr{B}_{\mathbb{R}^{k}}$ limitado. Com efeito, no caso geral podemos escrever $\mathrm{M}=\mathrm{M}_{0} \cup \bigcup_{i \in \mathbb{N}} g_{i}\left(E_{i}\right)$, com $\mathrm{M}_{0} \mathcal{H}^{k}$-nulo e, para cada $i \in \mathbb{N}, g_{i}\left(E_{i}\right)$ imagem Lipschitz regular definida pelo par $\left(g_{i}, E_{i}\right)$, com $E_{i} \in \mathscr{B}_{\mathbb{R}^{k}}$ limitado; ainda podemos tomar a última união disjunta, em vista da observação 4.8. Além disso: 
- Para cada $i \in \mathbb{N}, g_{i}\left(E_{i}\right)$ é $\mathcal{H}^{k}$-mensurável (pelo lema 3.7.i) e $\mathcal{H}^{k}\left(g_{i}\left(E_{i}\right)\right)<\infty$ (em vista da proposição 2.49.iii), do teorema 2.54 e do fato de que $E_{i}$ é limitado). Logo, pela proposição 2.26.i) e pela observação 2.24.ii), $\mathcal{H}^{k}\left\llcorner g_{i}\left(E_{i}\right)\right.$ é uma medida de Radon finita, e daí se conclui que $g_{i}\left(E_{i}\right)$ é $k$-retificável.

- Para cada $i \in \mathbb{N}$, admitindo provado o caso reduzido, $\mathrm{J}^{g_{i}\left(E_{i}\right)} f$ é uma função definida $\mathcal{H}^{k}$ q.s. em $g_{i}\left(E_{i}\right)$ e $\mathcal{H}^{k}$-mensurável. Como $\mathrm{T}_{x} g_{i}\left(E_{i}\right)=\mathrm{T}_{x} \mathrm{M}$ para $\mathcal{H}^{k}$-q.t. $x \in g_{i}\left(E_{i}\right)$ (em vista da proposição 4.19), conclui-se que $\mathrm{J}^{\mathrm{M}} f$ e $\mathrm{J}^{g_{i}\left(E_{i}\right)} f$ coincidem $\mathcal{H}^{k}$-q.s. em $g_{i}\left(E_{i}\right)$. Portanto, $\left.J^{\mathrm{M}} f\right|_{g_{i}\left(E_{i}\right)}$ é $\mathcal{H}^{k}$-mensurável; pela proposição 2.34 conclui-se que $\mathrm{J}^{\mathrm{M}} f$ é $\mathcal{H}^{k}$-mensurável.

- Para cada $i \in \mathbb{N}$, admitindo provado o caso reduzido, $N\left(\left.f\right|_{g_{i}\left(E_{i}\right)}\right): \mathbb{R}^{m} \rightarrow[0, \infty]$ é $\mathcal{H}^{k}$ mensurável. Além disso, $N\left(\left.f\right|_{\mathrm{M}_{0}}\right)$ é nula $\mathcal{H}^{k}$-q.s. em $\mathbb{R}^{m}$; com efeito, $f\left(\mathrm{M}_{0}\right)$ é $\mathcal{H}^{k}$-nulo (pois $f$ é Lipschitz e $\mathrm{M}_{0}$ é $\mathcal{H}^{k}$-nulo) e $N\left(\left.f\right|_{\mathrm{M}_{0}}\right)$ se anula no complementar de $f\left(\mathrm{M}_{0}\right)$. Em particular, $N\left(\left.f\right|_{\mathrm{M}_{0}}\right)$ é $\mathcal{H}^{k}$-mensurável. Como $\mathcal{H}^{0}$ é a medida de contagem, obtém-se 3 logo $N\left(\left.f\right|_{\mathrm{M}}\right)$ é o limite pontual de uma sequência de funções $\mathcal{H}^{k}$-mensuráveis, portanto é $\mathcal{H}^{k}$-mensurável em $\mathbb{R}^{m}$.

- Para cada $i \in \mathbb{N}$, pelo caso reduzido e pelo fato de que $\mathrm{J}^{\mathrm{M}} f$ e $\mathrm{J}^{g_{i}\left(E_{i}\right)} f$ coincidem $\mathcal{H}^{k}$-q.s. em $g_{i}\left(E_{i}\right)$,

$$
\int_{\mathbb{R}^{m}} N\left(\left.f\right|_{g_{i}\left(E_{i}\right)}\right) \mathrm{d} \mathcal{H}^{k}=\int_{g_{i}\left(E_{i}\right)} \mathrm{J}^{\mathrm{M}} f \mathrm{~d} \mathcal{H}^{k} .
$$

Daí, como $N\left(\left.f\right|_{\mathrm{M}}\right)=N\left(\left.f\right|_{\mathrm{M}_{0}}\right)+\sum_{i=1}^{\infty} N\left(\left.f\right|_{g_{i}\left(E_{i}\right)}\right)$ e $N\left(\left.f\right|_{\mathrm{M}_{0}}\right)$ é $\mathcal{H}^{k}$-nula, podemos somar em $i$ e aplicar o teorema da convergência monótona para concluir que

$$
\int_{\mathbb{R}^{m}} N\left(\left.f\right|_{\mathrm{M}}\right) \mathrm{d} \mathcal{H}^{k}=\int_{\mathrm{M}} \mathrm{J}^{\mathrm{M}} f \mathrm{~d} \mathcal{H}^{k}
$$

2) Suponha, pois, $\mathbf{M}=g(E)$ imagem Lipschitz regular $k$-dimensional definida pelo par $(g, E)$, com $E \in \mathscr{B}_{\mathbb{R}^{k}}$ limitado. Segue da observação 4.16 que existe o espaço tangente aproximado em todo $x \in \mathrm{M}$ e $\mathrm{T}_{x} \mathrm{M}=\mathrm{D} g\left(g^{-1}(x)\right) \cdot \mathbb{R}^{k}$. E, pelo lema 5.5, existe $D_{f} \subset \mathrm{M} \mathcal{H}^{k}$-mensurável com $\mathcal{H}^{k}\left(\mathrm{M} \backslash D_{f}\right)=0$ e tal que para todo $x \in D_{f}, f \circ g$ é derivável em $g^{-1}(x), f$ é tangencialmente diferenciável em $x$ com respeito a $\mathrm{M}$ e $\mathrm{D}^{\mathrm{M}} f(x)=\mathrm{D}(f \circ g)\left(g^{-1}(x)\right) \circ \mathrm{D} g\left(g^{-1}(x)\right)^{-1}: \mathrm{T}_{x} \mathrm{M} \rightarrow \mathbb{R}^{m}$. Como na demonstração do teorema 5.3, dado $x \in D_{f}$, fixamos uma isometria linear $\mathrm{T}_{x} \mathrm{M} \equiv \mathbb{R}^{k}$ (a qual não altera os jacobianos de $\mathrm{D} g\left(g^{-1}(x)\right) \in \mathrm{L}\left(\mathbb{R}^{k}, \mathrm{~T}_{x} \mathrm{M}\right)$ e $\mathrm{D}^{\mathrm{M}} f(x) \in \mathrm{L}\left(\mathrm{T}_{x} \mathrm{M}, \mathbb{R}^{m}\right)$ ); segue do lema 2.105.i) que:

$$
\begin{aligned}
\mathrm{J}(f \circ g)\left(g^{-1}(x)\right) & =\llbracket \mathrm{D}^{\mathrm{M}} f(x) \circ \mathrm{D} g\left(g^{-1}(x)\right) \rrbracket= \\
& =\llbracket \mathrm{D}^{\mathrm{M}} f(x) \rrbracket \llbracket \mathrm{D} g\left(g^{-1}(x)\right) \rrbracket= \\
& =\mathrm{J}^{\mathrm{M}} f(x) \mathrm{J} g\left(g^{-1}(x)\right) .
\end{aligned}
$$

Portanto, $\mathrm{J}^{\mathrm{M}} f: D_{f} \rightarrow \mathbb{R}$ é dada por

$$
\mathrm{J}^{\mathrm{M}} f=\frac{[\mathrm{J}(f \circ g)] \circ g^{-1}}{[\mathrm{~J} g] \circ g^{-1}}
$$

Como $g^{-1}:\left(\mathrm{M},\left.\sigma\left(\mathcal{H}^{k}\right)\right|_{\mathrm{M}}\right) \rightarrow\left(E,\left.\sigma\left(\mathcal{L}^{k}\right)\right|_{E}\right)$ é mensurável (pois, pelo fato de $g$ ser Lipschitz, segue do lema 3.7.i) que, para todo $A \subset E \mathcal{L}^{k}$-mensurável, $g(A)$ é $\mathcal{H}^{k}$-mensurável), e como J $f$ e $\mathrm{J}(f \circ g)$ são borelianas (em particular, são Lebesgue-mensuráveis), conclui-se de (5.5) que $\mathrm{J}^{\mathrm{M}} f$ é $\mathcal{H}^{k}$-mensurável, o que prova i).

Por outro lado, como $g: E \rightarrow \mathrm{M}=g(E)$ é uma bijeção, tem-se, para todo $y \in \mathbb{R}^{m}$,

$$
\mathcal{H}^{0}\left(\mathrm{M} \cap f^{-1}\{y\}\right)=\mathcal{H}^{0}\left(E \cap(f \circ g)^{-1}\{y\}\right) .
$$


Ou seja, $N\left(\left.f\right|_{M}\right)=N\left(\left.(f \circ g)\right|_{E}\right)$; aplicando-se o lema 3.7.ii) para a função Lipschitz $f \circ g$, conclui-se que $N\left(\left.f\right|_{\mathrm{M}}\right)$ é $\mathcal{H}^{k}$-mensurável, o que prova ii).

Finalmente, a fórmula da área 3.9 e o respectivo teorema de mudança de variáveis para funções Lipschitz em espaços euclidianos justificam o cálculo abaixo:

$$
\begin{aligned}
\int_{\mathbb{R}^{m}} \mathcal{H}^{0}\left(\mathrm{M} \cap f^{-1}\{y\}\right) \mathrm{d} \mathcal{H}^{k}(y) & \stackrel{(5.6)}{=} \int_{\mathbb{R}^{m}} \mathcal{H}^{0}\left(E \cap(f \circ g)^{-1}\{y\}\right) \stackrel{3.9}{=} \\
& =\int_{E} \mathrm{~J}(f \circ g) \mathrm{d} \mathcal{L}^{k} \stackrel{(5.4)}{=} \\
& =\int_{E}\left(\mathrm{~J}^{\mathrm{M}} f\right) \circ g \mathrm{~J} g \mathrm{~d} \mathcal{L}^{k} \stackrel{3.11}{=} \\
& =\int_{\mathrm{M}} \mathrm{J}^{\mathrm{M}} f \mathrm{~d} \mathcal{H}^{k}
\end{aligned}
$$

o que prova iii).

Corolário 5.8 (Teorema de Mudança de Variáveis). Sejam $\mathrm{M} \subset \mathbb{R}^{n}$ um conjunto $k$-localmente retificável e $f: \mathbb{R}^{n} \rightarrow \mathbb{R}^{m}$ Lipschitz, com $k \leq m$.

i) Se $g: \mathrm{M} \rightarrow[0, \infty]$ for $\mathcal{H}^{k}\llcorner\mathrm{M}-$ mensurável, então

$$
\int_{\mathrm{M}} g \mathrm{~J}^{\mathrm{M}} f \mathrm{~d} \mathcal{H}^{k}=\int_{\mathbb{R}^{m}}\left(\sum_{x \in \mathrm{M} \cap f^{-1}\{y\}} g(x)\right) \mathrm{d} \mathcal{H}^{k}(y),
$$

com o significado de que a integral do segundo membro faz sentido (i.e. o integrando é $\mathcal{H}^{k}$ mensurável) e vale a igualdade.

ii) Se $\left.f\right|_{\mathrm{M}}$ for 1-1 e $g: \operatorname{Im} f \rightarrow[0, \infty]$ for boreliana, então

$$
\int_{\operatorname{Im} f} g \mathrm{~d} \mathcal{H}^{k}=\int_{\mathrm{M}} g \circ f \mathrm{~J}^{\mathrm{M}} f \mathrm{~d} \mathcal{H}^{k} .
$$

Demonstração. Dada $g: \mathrm{M} \rightarrow[0, \infty] \mathcal{H}^{k}\llcorner\mathrm{M}$-mensurável, a proposição 2.33 garante a existência de uma sequência $\left(A_{i}\right)_{i \in \mathbb{N}}$ de subconjuntos $\mathcal{H}^{k}$-mensuráveis de $\mathrm{M}$ tal que

$$
g=\sum_{i=1}^{\infty} \frac{1}{i} \chi_{A_{i}} .
$$

Agora basta repetir o argumento do corolário 3.10, usando-se a versão da fórmula da área para conjuntos retificáveis 5.7 no lugar da fórmula da área 3.9, para concluir a parte i). A parte ii) é corolário da parte i), pelo mesmo argumento do corolário 3.11.

\subsection{Fórmula da Coárea para Conjuntos Retificáveis}

Teorema 5.9 (Fórmula da Coárea para aplicações Lipschitz em conjuntos retificáveis). Sejam $\mathrm{M} \subset \mathbb{R}^{n}$ um conjunto $k$-localmente retificável e $f: \mathbb{R}^{n} \rightarrow \mathbb{R}^{m}$ Lipschitz, com $k \geq m$. Então:

i) $\mathrm{J}^{\mathrm{M}} f$ é uma função definida $\mathcal{H}^{k}$-q.s. em $\mathrm{M}$ e $\mathcal{H}^{k}$-mensurável;

ii) a função de multiplicidade generalizada $N\left(\left.f\right|_{\mathrm{M}}\right): \mathbb{R}^{m} \rightarrow[0, \infty]$ dada por $y \mapsto \mathcal{H}^{k-m}(\mathrm{M} \cap$ $\left.f^{-1}\{y\}\right)$ é $\mathcal{L}^{m}$-mensurável; 
iii) $\int_{\mathbb{R}^{m}} \mathcal{H}^{k-m}\left(\mathrm{M} \cap f^{-1}\{y\}\right) \mathrm{d} \mathcal{L}^{m}(y)=\int_{\mathrm{M}} \mathrm{J}^{\mathrm{M}} f \mathrm{~d} \mathcal{H}^{k}$.

Na demonstração do teorema acima, usaremos as versões das fórmulas de área 3.9 e da coárea 3.23 em espaços euclidianos, bem como os lemas abaixo. Recorde, se necessário, as noções de integral superior e inferior introduzidas no preâmbulo em 2.36, bem como suas propriedades elementares em 2.37 .

Lema 5.10 ([9, 3.2.15]). Sejam $n>m$ e $f: \mathbb{R}^{n} \rightarrow \mathbb{R}^{m}$ Lipschitz. Então, para $\mathcal{L}^{m}$-q.t. $y \in \mathbb{R}^{m}$, $f^{-1}\{y\}$ é enumeravelmente $n-m$ retificável.

Lema 5.11. Sejam $M \subset \mathbb{R}^{n}$ um conjunto $k$-enumeravelmente retificável, $f: \mathbb{R}^{n} \rightarrow \mathbb{R}^{m}$ Lipschitz, $k \leq m$. Então $f(\mathrm{M})$ é $k$-enumeravelmente retificável.

Demonstração. Podemos tomar uma sequência $\left(g_{i}\right)_{i \in \mathbb{N}}$ de aplicações Lipschitz $\mathbb{R}^{k} \rightarrow \mathbb{R}^{n}$ e uma sequência de borelianos $\left(E_{i}\right)_{i \in \mathbb{N}}$ em $\mathbb{R}^{k}$ tais que $\mathrm{M}=\mathrm{M}_{0} \cup\left(\bigcup_{i \in \mathbb{N}} g_{i}\left(E_{i}\right)\right)$, com $\mathcal{H}^{k}\left(\mathrm{M}_{0}\right)=0$. Então $f(\mathrm{M})=f\left(\mathrm{M}_{0}\right) \cup\left(\bigcup_{i \in \mathbb{N}} f \circ g_{i}\left(E_{i}\right)\right)$, sendo:

1) $\mathcal{H}^{k}\left(f\left(\mathrm{M}_{0}\right)\right) \leq(\operatorname{Lip} f)^{k} \mathcal{H}^{k}\left(\mathrm{M}_{0}\right)=0$.

2) Para cada $i \in \mathbb{N}, f \circ g_{i}$ é Lipschitz. Em particular, $f \circ g_{i}\left(E_{i}\right)$ é $\mathcal{H}^{k}$-mensurável pelo lema 3.7.i), portanto, $f(\mathrm{M})$ é $\mathcal{H}^{k}$-mensurável (por ser uma união enumerável de $\mathcal{H}^{k}$-mensuráveis).

Corolário 5.12. Sejam $M \subset \mathbb{R}^{n}$ um conjunto $k$-enumeravelmente retificável e $f: \mathbb{R}^{n} \rightarrow \mathbb{R}^{m}$ Lipschitz, com $k>m$. Então existe um boreliano $R_{f} \subset \mathbb{R}^{m}$ tal que $\mathcal{L}^{m}\left(R_{f}^{c}\right)=0$ e, para todo $y \in R_{f}, \mathrm{M} \cap f^{-1}\{y\}$ é $(k-m)$-enumeravelmente retificável.

Demonstração. Podemos tomar uma sequência $\left(g_{i}\right)_{i \in \mathbb{N}}$ de aplicações Lipschitz $\mathbb{R}^{k} \rightarrow \mathbb{R}^{n}$ e uma sequência de borelianos $\left(E_{i}\right)_{i \in \mathbb{N}}$ em $\mathbb{R}^{k}$ tais que $\mathrm{M}=\mathrm{M}_{0} \cup\left(\bigcup_{i \in \mathbb{N}} g_{i}\left(E_{i}\right)\right)$, com $\mathcal{H}^{k}\left(\mathrm{M}_{0}\right)=0$. Tem-se:

1) Para cada $i \in \mathbb{N}$, pelo lema 5.10 aplicado a $f \circ g_{i}$, existe $R_{i} \subset \mathbb{R}^{m}$ boreliano com $\mathcal{L}^{m}\left(R_{i}^{c}\right)=0$ e, para todo $y \in R_{i},\left(f \circ g_{i}\right)^{-1}\{y\}$ é $(k-m)$-enumeravelmente retificável, logo $E_{i} \cap\left(f \circ g_{i}\right)^{-1}\{y\}$ também o é, pela proposição 4.3.ii). Portanto, para todo $y \in R_{i}, g_{i}\left(E_{i} \cap\left(f \circ g_{i}\right)^{-1}\{y\}\right)=$ $g_{i}\left(E_{i}\right) \cap f^{-1}\{y\}$ é $(k-m)$-enumeravelmente retificável pelo lema 5.11.

2) Segue da desigualdade de Eilenberg 3.18 que

$$
\int^{*} \mathcal{H}^{k-m}\left(\mathrm{M}_{0} \cap f^{-1}\{y\}\right) \mathrm{d} \mathcal{L}^{m}(y) \leq \frac{\alpha(k-m) \alpha(m)}{\alpha(k)}(\operatorname{Lip} f)^{m} \mathcal{H}^{k}\left(\mathrm{M}_{0}\right)=0 .
$$

Logo, da proposição 2.37, conclui-se que existe $R_{0} \subset \mathbb{R}^{m}$ boreliano tal que $\mathcal{L}^{m}\left(R_{0}^{c}\right)=0$ e, para todo $y \in R_{0}, \mathcal{H}^{k-m}\left(\mathrm{M}_{0} \cap f^{-1}\{y\}\right)=0$. Em particular, para todo $y \in R_{0}, \mathrm{M}_{0} \cap f^{-1}\{y\}$ é $(k-m)$-enumeravelmente retificável.

3) Tome $R_{f}:=\cap_{i \geq 0} R_{i} \in \mathscr{B}_{\mathbb{R}^{m}}$. Então $\mathcal{L}^{m}\left(R_{f}^{c}\right)=0$ e, para todo $y \in R_{f}, \mathrm{M}_{0} \cap f^{-1}\{y\}$ e $(\forall i \in$ $\mathbb{N}) g_{i}\left(E_{i}\right) \cap f^{-1}\{y\}$ são $(k-m)$-enumeravelmente retificáveis. Logo, para todo $y \in R_{f}$,

$$
\mathrm{M} \cap f^{-1}\{y\}=\left(\mathrm{M}_{0} \cap f^{-1}\{y\}\right) \cup\left(\bigcup_{i \in \mathbb{N}} g_{i}\left(E_{i}\right) \cap f^{-1}\{y\}\right)
$$

é $(k-m)$-enumeravelmente retificável, pela proposição 4.3.ii).

Prova do teorema 5.9. Podemos supor $k>m$, pois o caso $k=m$ segue da fórmula da área 5.7. 
1) Provemos que $\mathrm{J}^{\mathrm{M}} f$ é $\mathcal{H}^{k}$-mensurável. É suficiente considerar o caso em que $\mathrm{M}$ coincide com uma imagem Lipschitz regular $g(E)$ induzida por $g: \mathbb{R}^{k} \rightarrow \mathbb{R}^{n}$ Lipschitz e $E \in \mathscr{B}_{\mathbb{R}^{k}}$ limitado. O caso geral seguirá escrevendo $\mathrm{M}=\mathrm{M}_{0} \cup \bigcup_{i \in \mathbb{N}} g_{i}\left(E_{i}\right)$ com $\mathrm{M}_{0} \mathcal{H}^{k}$-nulo e, para cada $i \in \mathbb{N}, g_{i}\left(E_{i}\right)$ imagem Lipschitz regular induzida pelo $\operatorname{par}\left(g_{i}, E_{i}\right) \operatorname{com} E_{i} \in \mathscr{B}_{\mathbb{R}^{k}}$ limitado; a tese segue do caso reduzido e da proposição 2.34 , tendo em vista que $\mathrm{J}^{g_{i}\left(E_{i}\right)} f$ e $\mathrm{J}^{\mathrm{M}} f$ coincidem $\mathcal{H}^{k}$-q.s. em $g_{i}\left(E_{i}\right)$ pela proposição 4.19 .

Suponha, pois, $\mathrm{M}=g(E)$ imagem Lipschitz regular induzida pelo par $(g, E)$, com $E \in \mathscr{B}_{\mathbb{R}^{k}}$ limitado.

Do lema 5.5 e da observação 4.16 segue que, para $\mathcal{H}^{k}$-q.t. $x \in \mathrm{M}, f \circ g$ é derivável em $g^{-1}(x)$, o espaço tangente aproximado a $\mathrm{M}$ em $x$ existe e coincide com $\mathrm{D} g\left(g^{-1}(x)\right) \cdot \mathbb{R}^{k}, f$ é M-tangencialmente diferenciável em $x$ e $\mathrm{D}(f \circ g)\left(g^{-1}(x)\right)=\mathrm{D}^{\mathrm{M}} f(x) \circ \mathrm{D} g\left(g^{-1}(x)\right)$. Num tal $x$, tem-se, pela definição 2.102 ,

$$
\mathrm{J}^{\mathrm{M}} f(x)=\llbracket \mathrm{D}^{\mathrm{M}} f(x) \rrbracket=\sqrt{\operatorname{det} \mathrm{D}^{\mathrm{M}} f(x) \circ \mathrm{D}^{\mathrm{M}} f(x)^{*}} .
$$

Assim, como $\sqrt{ } \cdot$ e det são funções contínuas, basta verificar que as entradas da matriz da transformação linear $\mathrm{D}^{\mathrm{M}} f(x) \circ \mathrm{D}^{\mathrm{M}} f(x)^{*}$ na base canônica $\left(e_{1}, \ldots, e_{m}\right)$ de $\mathbb{R}^{m}$ são, como funções de $x$, funções $\mathcal{H}^{k}$-mensuráveis definidas $\mathcal{H}^{k}$-q.s. em M. De fato, por um cálculo direto envolvendo álgebra linear elementar, a $(i, j)$-ésima entrada da referida matriz é dada por

$$
\left\langle\mathrm{D}^{\mathrm{M}} f(x) \circ \mathrm{D}^{\mathrm{M}} f(x)^{*} \cdot e_{i}, e_{j}\right\rangle=\left\langle\mathrm{D}^{\mathrm{M}} f(x)^{*} \cdot e_{i}, \mathrm{D}^{\mathrm{M}} f(x)^{*} \cdot e_{j}\right\rangle=\beta_{i}(x)^{\mathrm{t}} \cdot G(x) \cdot \beta_{j}(x),
$$

onde, denotando por $\left(f_{1}, \ldots, f_{k}\right)$ a base canônica de $\mathbb{R}^{k}$ :

- Para $1 \leq i \leq m, \beta_{i}(x)$ é a matriz coluna $k \times 1$ cuja $j$-ésima linha é dada por

$$
\left\langle\mathrm{D}(f \circ g)\left(g^{-1}(x)\right) \cdot f_{j}, e_{i}\right\rangle .
$$

Como $g^{-1}: \mathrm{M} \rightarrow \mathbb{R}^{k}$ é $\mathcal{H}^{k}$-mensurável (em vista do lema 3.7.i aplicado a $g$; ou, alternativamente, podemos supor que $g: E \rightarrow \mathrm{M}$ é bi-Lipschitz, em vista do teorema de estrutura 4.7 , e nesse caso $g^{-1}$ é boreliana) e $\mathrm{D}(f \circ g)$ é boreliana (definida no complementar em $\mathbb{R}^{k}$ de um boreliano $\mathcal{L}^{k}$-nulo), conclui-se que $\beta_{i}$ é uma função $\mathcal{H}^{k}$-mensurável definida $\mathcal{H}^{k}$-q.s. em M.

- $G(x)$ é a inversa da matriz grammiana dos vetores $\mathrm{D} g\left(g^{-1}(x)\right) \cdot f_{1}, \ldots, \mathrm{D} g\left(g^{-1}(x)\right) \cdot f_{k} \in$ $\mathrm{T}_{x} \mathrm{M} \subset \mathbb{R}^{n}$, i.e. a inversa da matriz $k \times k$ cuja $(i, j)$-ésima entrada é

$$
\left\langle\mathrm{D} g\left(g^{-1}(x)\right) \cdot f_{i}, \mathrm{D} g\left(g^{-1}(x)\right) \cdot f_{j}\right\rangle \text {. }
$$

Como a inversão de matrizes $\mathrm{GL}(k) \rightarrow \mathrm{GL}(k)$ é contínua, $\mathrm{D} g$ é boreliana (definida no complementar em $\mathbb{R}^{k}$ de um boreliano $\mathcal{L}^{k}$-nulo) e $g^{-1}: \mathrm{M} \rightarrow \mathbb{R}^{k}$ é $\mathcal{H}^{k}$-mensurável, concluise que $G$ é uma função $\mathcal{H}^{k}$-mensurável definida $\mathcal{H}^{k}$-q.s. em $\mathrm{M}$.

Portanto, de (5.7), conclui-se que a matriz de $x \mapsto \mathrm{D}^{\mathrm{M}} f(x) \circ \mathrm{D}^{\mathrm{M}} f(x)^{*}$ é uma função $\mathcal{H}^{k}$ mensurável definida $\mathcal{H}^{k}$-q.s. em M, como afirmado. Está concluída, assim, a prova da parte i).

2) $\mathrm{A} 3$

Com efeito, assumindo provado o caso em que $\mathcal{H}^{k}(\mathrm{M})<\infty$, no caso geral podemos tomar, em vista da proposição 4.3.iv), uma sequência crescente $\left(\mathrm{M}_{i}\right)_{i \in \mathbb{N}}$ de $\mathcal{H}^{k}$-mensuráveis com $(\forall i \in$ $\mathbb{N}) \mathcal{H}^{k}\left(\mathrm{M}_{i}\right)<\infty$ e $\cup_{i \in \mathbb{N}} \mathrm{M}_{i}=\mathrm{M}$. Em particular, todos os $\mathrm{M}_{i}$ 's são $k$-retificáveis e o caso reduzido se aplica; ou seja, ii) e iii) valem com $\mathrm{M}_{i}$ no lugar de $\mathrm{M}$, para todo $i \in \mathbb{N}$. Como, para todo $y \in \mathbb{R}^{m}, \mathrm{M}_{i} \cap f^{-1}\{y\}$ cresce para $\mathrm{M} \cap f^{-1}\{y\}$, segue da observação 2.10 que $N\left(\left.f\right|_{\mathrm{M}_{i}}\right)$ cresce pontualmente para $N\left(\left.f\right|_{M}\right)$. Portanto, a última função é $\mathcal{L}^{m}$-mensurável, e a igualdade em iii) segue do teorema da convergência monótona e do fato de que, pela proposição 4.19, para todo $i \in \mathbb{N}, \mathrm{J}^{\mathrm{M}} f$ coincide $\mathcal{H}^{k}$-q.s. com $\mathrm{J}^{\mathrm{M}_{i}} f$ em $\mathrm{M}_{i}$. 
3) Assuma, pois, $\mathcal{H}^{k}(\mathrm{M})<\infty$. Note que $\int_{\mathrm{M}} \mathrm{J}^{\mathrm{M}} f \mathrm{~d} \mathcal{H}^{k}$ é finita, pois $\mathrm{J}^{\mathrm{M}} f$ é limitada por $(\operatorname{Lip} f)^{k}$. Além disso, o corolário 5.12 garante a existência de um boreliano $R_{f} \subset \mathbb{R}^{m}$ tal que $\mathcal{L}^{m}\left(R_{f}^{c}\right)=0$ e, para todo $y \in R_{f}, \mathrm{M} \cap f^{-1}\{y\}$ é $(k-m)$-enumeravelmente retificável.

Fixe $t>1$. Em vista do teorema 4.7, podemos tomar uma sequência $\left(g_{i}\right)_{i \in \mathbb{N}}$ de aplicações Lipschitz $\mathbb{R}^{k} \rightarrow \mathbb{R}^{n}$ e uma sequência de borelianos limitados $\left(E_{i}\right)_{i \in \mathbb{N}}$ em $\mathbb{R}^{k}$ tais que:

- Para cada $i \in \mathbb{N}$, o par $\left(g_{i}, E_{i}\right)$ define uma imagem Lipschitz regular $\mathrm{M}_{i}:=g_{i}\left(E_{i}\right)$ no sentido da definição 4.5.

- $\mathrm{M}=\mathrm{M}_{0} \cup\left(\bigcup_{i \in \mathbb{N}} g_{i}\left(E_{i}\right)\right)$, com $\mathcal{H}^{k}\left(\mathrm{M}_{0}\right)=0$; podemos assumir que essa união é disjunta, pela observação 4.8 .

- Para todo $i \in \mathbb{N}$, todos $x, y \in E_{i}$ e todo $v \in \mathbb{R}^{k}$,

$$
\begin{aligned}
& t^{-1}\|x-y\| \leq\left\|g_{i}(x)-g_{i}(y)\right\| \leq t\|x-y\|, \\
& t^{-1}\|v\| \leq\left\|\mathrm{D} g_{i}(x) \cdot v\right\| \leq t\|v\|, \\
& t^{-k} \leq \mathrm{J} g_{i}(x) \leq t^{k}
\end{aligned}
$$

Portanto,

$$
\left.\operatorname{Lip} g_{i}\right|_{E_{i}} \leq t,\left.\quad \operatorname{Lip} g_{i}\right|_{E_{i}} ^{-1} \leq t, \quad\left\|\mathrm{D} g_{i}(x)\right\| \leq t, \quad\left\|\mathrm{D} g_{i}(x)^{-1}\right\| \leq t
$$

Segue da desigualdade de Eilenberg 3.18 que,

$$
\int^{*} \mathcal{H}^{k-m}\left(\mathrm{M}_{0} \cap f^{-1}\{y\}\right) \mathrm{d} \mathcal{L}^{m}(y) \leq \frac{\alpha(k-m) \alpha(m)}{\alpha(k)}(\operatorname{Lip} f)^{m} \mathcal{H}^{k}\left(\mathrm{M}_{0}\right)=0 .
$$

Assim, diminuindo o boreliano $R_{f}$, se necessário, podemos supor que $\mathcal{H}^{k-m}\left(\mathrm{M}_{0} \cap f^{-1}\{y\}\right)=0$ para todo $y \in R_{f}$. Além disso, em vista do lema 5.10, podemos diminuir o boreliano $R_{f}$ ainda mais, se necessário, de modo a garantir que, para todo $i \in \mathbb{N}$ e todo $y \in R_{f}, E_{i}(y):=E_{i} \cap(f \circ$ $\left.g_{i}\right)^{-1}\{y\}$ é $(k-m)$-enumeravelmente retificável. Portanto, pelo lema 5.11, para cada $y \in R_{f}$ e cada $i \in \mathbb{N}, g_{i}\left(E_{i}(y)\right)=\mathrm{M}_{i} \cap f^{-1}\{y\}$ é $k-m$-enumeravelmente retificável e, em particular, $\mathcal{H}^{k-m}$-mensurável. Assim, para cada $y \in R_{f}, \mathrm{M} \cap f^{-1}\{y\}$ se escreve como união disjunta dos $\mathcal{H}^{k-m}$-mensuráveis $\mathrm{M}_{0} \cap f^{-1}\{y\}$ e $\mathrm{M}_{i} \cap f^{-1}\{y\}, i \in \mathbb{N}$. Daí, para todo $y \in R_{f}$,

$$
\begin{aligned}
\mathcal{H}^{k-m}\left(\mathrm{M} \cap f^{-1}\{y\}\right) & =\underbrace{\mathcal{H}^{k-m}\left(\mathrm{M}_{0} \cap f^{-1}\{y\}\right)}_{=0}+\sum_{i=1}^{\infty} \mathcal{H}^{k-m}\left(\mathrm{M}_{i} \cap f^{-1}\{y\}\right)= \\
& =\sum_{i=1}^{\infty} \mathcal{H}^{k-m}\left(\mathrm{M}_{i} \cap f^{-1}\{y\}\right) .
\end{aligned}
$$

4) Para todo $i \in \mathbb{N}$, tem-se:

- Segue do lema 5.5 e da observação 4.16 que, para $\mathcal{L}^{k}$-q.t. $z \in E_{i}, f \circ g_{i}$ é derivável em $z$, o espaço tangente aproximado a $\mathrm{M}_{i}=g_{i}\left(E_{i}\right)$ em $g_{i}(z)$ existe e coincide com $\mathrm{D} g_{i}(z) \cdot \mathbb{R}^{k}, f$ é $\mathrm{M}_{i}$-tangencialmente diferenciável em $g_{i}(z)$ e $\mathrm{D}\left(f \circ g_{i}\right)(z)=\mathrm{D}^{\mathrm{M}_{i}} f\left(g_{i}(z)\right) \circ \mathrm{D} g_{i}(z)$.

- Da proposição 4.19 segue que os espaços tangentes aproximados a $\mathrm{M}_{i}$ e a $\mathrm{M}$ coincidem $\mathcal{H}^{k}$-q.s. em $\mathrm{M}_{i}$; ou, equivalentemente, tendo em vista que $\left.g_{i}\right|_{E_{i}}: E_{i} \rightarrow \mathrm{M}_{i}$ é bi-Lipschitz, para $\mathcal{L}^{k}$-q.t. $z \in E_{i}, \mathrm{~T}_{g_{i}(z)} \mathrm{M}_{i}=\mathrm{T}_{g_{i}(z)} \mathrm{M}$.

- Dos dois itens anteriores conclui-se que, para $\mathcal{L}^{k}$-q.t. $z \in E_{i}, f \circ g_{i}$ é derivável em $z$, o espaço tangente aproximado a $\mathrm{M}$ em $g_{i}(z)$ existe e coincide com $\mathrm{D} g_{i}(z) \cdot \mathbb{R}^{k}, f$ é $\mathrm{M}$-tangencialmente diferenciável em $g_{i}(z)$ e $\mathrm{D}\left(f \circ g_{i}\right)(z)=\mathrm{D}^{\mathrm{M}} f\left(g_{i}(z)\right) \circ \mathrm{D} g_{i}(z)$. Para tais $z$, tomando a adjunta em ambos os membros da última igualdade segue $\mathrm{D}\left(f \circ g_{i}\right)(z)^{*}=\mathrm{D} g_{i}(z)^{*} \circ \mathrm{D}^{\mathrm{M}} f\left(g_{i}(z)\right)^{*}$; daí, identificando o espaço tangente aproximado $\mathrm{T}_{g_{i}(z)} \mathrm{M}$ com $\mathbb{R}^{k}$ através de alguma isometria 
linear (o que não altera o jacobiano de $\mathrm{D}^{\mathrm{M}} f\left(g_{i}(z)\right): \mathrm{T}_{g_{i}(z)} \mathrm{M} \rightarrow \mathbb{R}^{m}$ nem o de $\mathrm{D} g_{i}(z): \mathbb{R}^{k} \rightarrow$ $\left.\mathrm{T}_{g_{i}(z)} \mathrm{M}=\mathrm{D} g_{i}(z) \cdot \mathbb{R}^{k}\right)$, e tendo em vista que $\left\|\mathrm{D} g_{i}(z)^{*}\right\|=\left\|\mathrm{D} g_{i}(z)\right\| \leq t$ e $\left\|\left[\mathrm{D} g_{i}(z)^{*}\right]^{-1}\right\|=$ $\left\|\mathrm{D} g_{i}(z)^{-1}\right\| \leq t$, podemos aplicar o lema $3.22 \mathrm{com} R=\mathrm{D} g_{i}(z)^{*}$ e $T=\mathrm{D}^{\mathrm{M}} f\left(g_{i}(z)\right)^{*}$ para concluir que

$$
t^{-k} \mathrm{~J}^{\mathrm{M}} f\left(g_{i}(z)\right) \leq \mathrm{J}\left(f \circ g_{i}\right)(z) \leq t^{k} \mathrm{~J}^{\mathrm{M}} f\left(g_{i}(z)\right) .
$$

- Para todo $y \in \mathbb{R}^{m},\left.g_{i}\right|_{E_{i}}$ leva $E_{i}(y)=E_{i} \cap\left(f \circ g_{i}\right)^{-1}\{y\}$ bijetivamente sobre $\mathrm{M}_{i} \cap f^{-1}\{y\}$ e $\left.\operatorname{Lip} g_{i}\right|_{E_{i}} \leq t,\left.\operatorname{Lip} g_{i}\right|_{E_{i}} ^{-1} \leq t$. Portanto, para todo $y \in \mathbb{R}^{m}$, segue da proposição 2.49.iii) que

$$
t^{-(k-m)} \mathcal{H}^{k-m}\left(E_{i}(y)\right) \leq \mathcal{H}^{k-m}\left(\mathrm{M}_{i} \cap f^{-1}\{y\}\right) \leq t^{k-m} \mathcal{H}^{k-m}\left(E_{i}(y)\right) .
$$

Finalmente, podemos estimar, usando as versões da fórmula da área (FA) 3.9 e da coárea (FCA) 3.23 para aplicações Lipschitz em espaços euclidianos:

$$
\begin{aligned}
& t^{-(3 k-m)} \int_{=g_{i}\left(E_{i}\right)}^{\mathrm{M}_{i}} \mathrm{~J}^{\mathrm{M}} f \mathrm{~d} \mathcal{H}^{k} \stackrel{\mathrm{FA}}{=} t^{-(3 k-m)} \int_{E_{i}} \mathrm{~J}^{\mathrm{M}} f \circ g_{i} \mathrm{~J} g_{i} \mathrm{~d} \mathcal{L}^{k} \stackrel{(5.10),(5.8)}{\leq} \\
& \leq t^{-(k-m)} \int_{E_{i}} \mathrm{~J}\left(f \circ g_{i}\right) \mathrm{d} \mathcal{L}^{k} \stackrel{\mathrm{FCA}}{=} \\
& =t^{-(k-m)} \int \mathcal{H}^{k-m}(\underbrace{E_{i} \cap\left(f \circ g_{i}\right)^{-1}\{y\}}_{=E_{i}(y)}) \mathrm{d} \mathcal{L}^{m}(y) \stackrel{(5.11)}{\leq} \\
& \leq \int_{*} \mathcal{H}^{k-m}\left(\mathrm{M}_{i} \cap f^{-1}\{y\}\right) \mathrm{d} \mathcal{L}^{m}(y) \leq \\
& \leq \int^{*} \mathcal{H}^{k-m}\left(\mathrm{M}_{i} \cap f^{-1}\{y\}\right) \mathrm{d} \mathcal{L}^{m}(y) \stackrel{(5.11)}{\leq} \\
& \leq t^{k-m} \int \mathcal{H}^{k-m}\left(E_{i} \cap\left(f \circ g_{i}\right)^{-1}\{y\}\right) \mathrm{d} \mathcal{L}^{m}(y) \stackrel{\mathrm{FCA}}{=} \\
& =t^{k-m} \int_{E_{i}} \mathrm{~J}\left(f \circ g_{i}\right) \mathrm{d} \mathcal{L}^{k} \stackrel{(5.10),(5.8)}{\leq} \\
& \leq t^{3 k-m} \int_{E_{i}} \mathrm{~J}^{\mathrm{M}} f \circ g_{i} \mathrm{~J} g_{i} \mathrm{~d} \mathcal{L}^{k} \stackrel{\mathrm{FA}}{=} \\
& =t^{3 k-m} \int_{\mathrm{M}_{i}} \mathrm{~J}^{\mathrm{M}} f \mathrm{~d} \mathcal{H}^{k} \text {. }
\end{aligned}
$$

Note que, nas estimativas acima, tomamos as integrais inferior e superior de $\mathcal{H}^{k-m}\left(\mathrm{M}_{i} \cap\right.$ $\left.f^{-1}\{\cdot\}\right)$, ao invés de tomar a integral usual contra a medida de Lebesgue, porque não sabemos, a priori, se esta função é $\mathcal{L}^{m}$-mensurável.

Destaquemos, em particular,

$$
\begin{aligned}
t^{-(3 k-m)} \int_{\mathrm{M}_{i}} \mathrm{~J}^{\mathrm{M}} f \mathrm{~d} \mathcal{H}^{k} & \leq \int_{*} \mathcal{H}^{k-m}\left(\mathrm{M}_{i} \cap f^{-1}\{y\}\right) \mathrm{d} \mathcal{L}^{m}(y) \leq \\
& \leq \int^{*} \mathcal{H}^{k-m}\left(\mathrm{M}_{i} \cap f^{-1}\{y\}\right) \mathrm{d} \mathcal{L}^{m}(y) \leq \\
& \leq t^{3 k-m} \int_{\mathrm{M}_{i}} \mathrm{~J}^{\mathrm{M}} f \mathrm{~d} \mathcal{H}^{k} .
\end{aligned}
$$

5) Para $i=0$, temos $\mathcal{H}^{k}\left(\mathrm{M}_{0}\right)=0$, logo $\int_{\mathrm{M}_{0}} \mathrm{~J}^{\mathrm{M}} f \mathrm{~d} \mathcal{H}^{k}=0$. E, conforme obtido na parte 3), 


$$
\begin{aligned}
& \int_{*} \mathcal{H}^{k-m}\left(\mathrm{M}_{0} \cap f^{-1}\{y\}\right) \mathrm{d} \mathcal{L}^{m}(y)=\int^{*} \mathcal{H}^{k-m}\left(\mathrm{M}_{0} \cap f^{-1}\{y\}\right) \mathrm{d} \mathcal{L}^{m}(y)=0 . \text { Portanto, } \\
& \int_{\mathrm{M}_{0}} \mathrm{~J}^{\mathrm{M}} f \mathrm{~d} \mathcal{H}^{k}=\int_{*} \mathcal{H}^{k-m}\left(\mathrm{M}_{0} \cap f^{-1}\{y\}\right) \mathrm{d} \mathcal{L}^{m}(y)=\int^{*} \mathcal{H}^{k-m}\left(\mathrm{M}_{0} \cap f^{-1}\{y\}\right) \mathrm{d} \mathcal{L}^{m}(y)=0 .
\end{aligned}
$$

De (5.12), (5.13) e do teorema da convergência monótona (TCM), obtém-se:

$$
\begin{aligned}
& t^{-(3 k-m)} \int_{\mathrm{M}} \mathrm{J}^{\mathrm{M}} f \mathrm{~d} \mathcal{H}^{k} \stackrel{\mathrm{T}}{=} t^{-(3 k-m)} \sum_{i=0}^{\infty} \int_{\mathrm{M}_{i}} \mathrm{~J}^{\mathrm{M}} f \mathrm{~d} \mathcal{H}^{k} \stackrel{(5.13),(5.12)}{\leq} \\
& \leq \sum_{i=0}^{\infty} \int_{*} \mathcal{H}^{k-m}\left(\mathrm{M}_{i} \cap f^{-1}\{y\}\right) \mathrm{d} \mathcal{L}^{m}(y) \stackrel{\text { prop. 2.37.v }}{\leq} \\
& \leq \int_{*} \underbrace{\sum_{i=0}^{\infty} \mathcal{H}^{k-m}\left(\mathrm{M}_{i} \cap f^{-1}\{y\}\right)}_{=\mathcal{H}^{k-m}\left(\mathrm{M} \cap f^{-1}\{y\}\right) \forall y \in R_{f} \text { por }(5.9)} \mathrm{d} \mathcal{L}^{m}(y) \stackrel{\mathcal{L}^{m}\left(R_{f}^{c}\right)=0,5.13}{=} \\
& =\int_{*} \mathcal{H}^{k-m}\left(\mathrm{M} \cap f^{-1}\{y\}\right) \mathrm{d} \mathcal{L}^{m}(y) \leq \\
& \leq \int^{*} \mathcal{H}^{k-m}\left(\mathrm{M} \cap f^{-1}\{y\}\right) \mathrm{d} \mathcal{L}^{m}(y) \stackrel{\mathcal{L}^{m}\left(R_{f}^{c}\right)=0,5.13}{=} \\
& =\int^{*} \sum_{i=0}^{\infty} \mathcal{H}^{k-m}\left(\mathrm{M}_{i} \cap f^{-1}\{y\}\right) \mathrm{d} \mathcal{L}^{m}(y) \stackrel{\text { prop. 2.37.v }}{\leq} \\
& \leq \sum_{i=0}^{\infty} \int^{*} \mathcal{H}^{k-m}\left(\mathrm{M}_{i} \cap f^{-1}\{y\}\right) \mathrm{d} \mathcal{L}^{m}(y) \stackrel{(5.13),(5.12)}{\leq} \\
& \leq t^{3 k-m} \sum_{i=0}^{\infty} \int_{\mathrm{M}_{i}} \mathrm{~J}^{\mathrm{M}} f \mathrm{~d} \mathcal{H}^{k} \stackrel{\mathrm{T}}{=} \\
& =t^{3 k-m} \int_{\mathrm{M}} \mathrm{J}^{\mathrm{M}} f \mathrm{~d} \mathcal{H}^{k} \text {. }
\end{aligned}
$$

Destaquemos, em particular,

$$
\begin{aligned}
t^{-(3 k-m)} \int_{\mathrm{M}} \mathrm{J}^{\mathrm{M}} f \mathrm{~d} \mathcal{H}^{k} & \leq \int_{*} \mathcal{H}^{k-m}\left(\mathrm{M} \cap f^{-1}\{y\}\right) \mathrm{d} \mathcal{L}^{m}(y) \leq \\
& \leq \int^{*} \mathcal{H}^{k-m}\left(\mathrm{M} \cap f^{-1}\{y\}\right) \mathrm{d} \mathcal{L}^{m}(y) \leq \\
& \leq t^{3 k-m} \int_{\mathrm{M}} \mathrm{J}^{\mathrm{M}} f \mathrm{~d} \mathcal{H}^{k} .
\end{aligned}
$$

Como $t>1$ foi fixado arbitrariamente e $k>m$, tomando $t \downarrow 1$ em (5.14) obtém-se:

$$
\int_{*} \mathcal{H}^{k-m}\left(\mathrm{M} \cap f^{-1}\{y\}\right) \mathrm{d} \mathcal{L}^{m}(y)=\int^{*} \mathcal{H}^{k-m}\left(\mathrm{M} \cap f^{-1}\{y\}\right) \mathrm{d} \mathcal{L}^{m}(y)=\int_{\mathrm{M}} \mathrm{J}^{\mathrm{M}} f \mathrm{~d} \mathcal{H}^{k}<\infty .
$$

Logo, da proposição 2.37.i), conclui-se que valem as afirmações ii) e iii) do enunciado do teorema, i.e. $y \mapsto \mathcal{H}^{k-m}\left(\mathrm{M} \cap f^{-1}\{y\}\right)$ é $\mathcal{L}^{m}$-mensurável e

$$
\int \mathcal{H}^{k-m}\left(\mathrm{M} \cap f^{-1}\{y\}\right) \mathrm{d} \mathcal{L}^{m}(y)=\int_{\mathrm{M}} \mathrm{J}^{\mathrm{M}} f \mathrm{~d} \mathcal{H}^{k},
$$

o que conclui a demonstração. 
Observação 5.13. Foi usado no argumento o seguinte fato: se $\mu$ medida exterior em $X$ e $f, g$ : $X \rightarrow[0, \infty]$ (não assumimos que sejam $\mu$-mensuráveis) coincidem $\mu$-q.s., então $\int_{*} f \mathrm{~d} \mu=\int_{*} g \mathrm{~d} \mu \mathrm{e}$ $\int^{*} f \mathrm{~d} \mu=\int^{*} g \mathrm{~d} \mu$. Isso é trivial, pois, se $\varphi \in \mathrm{L}^{+}$é simples estendida, então $\varphi \leq f$ (resp. $\geq f$ ) a.e. se, e somente se, $\varphi \leq g($ resp. $\geq g)$ a.e. 


\section{Referências Bibliográficas}

[1] A. S. Besicovitch, "On the fundamental geometrical properties of linearly measurable plane sets of points (III)", journal: Mathematische Annalen volume 116, pages349-357, (1939). https://doi.org/10.1007/BF01597361 51

[2] B. Esmayli and P. Hajlasz, "The Coarea Inequality", 2020, https://arxiv.org/abs/2006.00419. 37

[3] E. DE Giorgi, Su una teoria generale della misura $(r-1)$-dimensionale in uno spazio ad $r$ dimensioni, Annali di Matematica Pura ed Applicata, 36 (1954), pp. 191-213. 1

[4] Morrey, Charles B., Jr.; A Class of Representations of Manifolds. Part I. Amer. J. Math. 55 (1933), no. 1-4, 683-707. 1

[5] F. Maggi, "Set of finite perimeter and geometric variational problems, An introduction to Geometric Measure Theory" Cambridge U Press, 2012. 3, 20, 21, 51

[6] Gerald B. Folland, "Real analysis", second ed., Pure and Applied Mathematics (New York), John Wiley and Sons, Inc., New York, 1999, Modern techniques and their applications, A Wiley- Interscience Publication. MR 1681462 3, 4, 8, 9, 19, 27, 34

[7] Gláucio Terra. Introduction to Geometric Measure Theory (unpublished lecture notes), 2019. URL: https://www.ime.usp.br/ glaucio/mat6704/textos/GMTLecureNotes.pdf. 3, 5, 7, 10, $11,13,14,15,16,17,18,19,20,21,22$

[8] H. Federer, "Curvature measures,"Transactions of the American Mathematical Society, Transactions of the American Mathematical Society, Vol.93, No.3, 93(3) : 418-491, doi: 10.2307/1993504, (1959). 1

[9] H. Federer, "Geometric Measure Theory," Springer. 1969. 1, 2, 3, 4, 6, 7, 8, 9, 10, 11, 12, 13, $14,15,16,21,26,34,37,48,51,56,75$

[10] I. Chavel, Isoperimetric inequalities, vol. 145 of Cambridge Tracts in Mathematics, Cambridge University Press, Cambridge, 2001. Differential geometric and analytic perspectives. 36

[11] H. Federer "The $(\phi, k)$ Rectifiable subsets of $n$ space", journal: Trans. Amer. Math. Soc. 62, 114-192, (1947). https://doi.org/10.1090/S0002-9947-1947-0022594-3 51

[12] L.C. Evans, R.F. Gariepy "Measure Theory and fine properties of functions," CRC Press Taylor \& Francis Group, 2015. 3, 4, 6, 11, 13, 14, 15, 20, 21, 22, 38

[13] L. Simon, "Introduction to Geometric Measure Theory," Stanford University, 2014. 3, 15, 16, 17,18

[14] M. B. Karmanova "Coarea Formula for Functions on 2-Step Carnot Groups with SubLorentzian Structure", journal: Doklady Mathematics volume 101, pages129-131, (2020). https://link.springer.com/article/10.1134/S1064562420020131 2 
[15] Michael E. Taylor,"Measure Theory and Integration," v.76, 2006 by the American Mathematical Society. 34

[16] P. Gladbach, H. Olbermann "Coarea formulae and chain rules for the Jacobian determinant in fractional Sobolev spaces", arXiv:1903.07420(v2), (2019). https://arxiv.org/abs/1903.07420 2

[17] P. Mattila, "Geometry of Sets and Measures in Euclidean Spaces," Cambridge U Press, 1995. $12,15,51$

[18] Walter Rudin, "Principles of mathematical analysis."University of Wisconsin, (1976). 3, 9, 27 